\title{
Electric and heat transport in two-channel Kondo systems
}

\author{
G.A.R. van Dalum
}


PhD thesis, Utrecht University, September 2020

Printed by ProefschriftMaken || www.proefschriftmaken.nl ISBN: 978-90-393-7320-0

About the cover: The illustration on the cover depicts a marble coming in from the left and hitting a set of stationary marbles. The incoming marble comes to a halt and therefore becomes one of the stationary marbles, while one of the previously stationary marbles is bounced off to the right. This is a metaphor for the most important transport process discussed in this thesis, consisting of a particle entering a central region from one lead and a different particle subsequently leaving this central region to another lead. Cover art and design by M.J. van Dalum. 


\section{Electric and heat transport in two-channel Kondo systems}

\section{Transport van elektrische lading en warmte in twee-kanaals Kondo systemen}

(met een samenvatting in het Nederlands)

\section{Proefschrift}

ter verkrijging van de graad van doctor aan de Universiteit Utrecht op gezag van de rector magnificus, prof. dr. H.R.B.M. Kummeling, ingevolge het besluit van het college voor promoties in het openbaar te verdedigen op woensdag 23 september 2020 des middags te 12.45 uur

door

\section{Gerwin Antony Ronald van Dalum}

geboren op 28 mei 1991 te Nieuwegein 
Promotor: Prof. dr. ir. H.T.C. Stoof

Copromotor: Dr. L. Fritz

Dit proefschrift werd mogelijk gemaakt met financiële steun van de Stichting Nederlandse Wetenschappelijk Onderzoek Instituten (NWO-I, voorheen FOM), die deel uitmaakt van de Nederlandse Organisatie voor Wetenschappelijk Onderzoek (NWO). 


\section{Contents}

Preface vii

List of publications $\quad$ ix

1 Introduction 1

1.1 Interacting fermions . . . . . . . . . . . . . . . 2

1.1.1 Fermi liquid theory . . . . . . . . . . . . . . . 2

1.1.2 Non-Fermi liquid physics . . . . . . . . . . . . . 7

1.2 Outline ...................... . . 7

$\begin{array}{lll}2 & \text { The Kondo effect } & 9\end{array}$

2.1 Microscopic origin of the Kondo model . . . . . . . . . . . . . 10

2.2 Resistance minimum and the Kondo problem . . . . . . . . . 13

2.3 Poor man's scaling and the renormalization group . . . . . . 15

2.4 Physics of the strong coupling fixed point . . . . . . . . . 20

3 The two-channel Kondo model 23

3.1 Model Hamiltonian and its ground states . . . . . . . . . . 23

3.2 First experimental realization . . . . . . . . . . . . . . 26

3.3 The charge two-channel Kondo model . . . . . . . . . . . . . 28

3.4 Two-channel physics . . . . . . . . . . . . . . . . . 31

3.5 Exactly solvable point of the model . . . . . . . . . . . . . . . 34

3.6 Applicability of the Emery-Kivelson point . . . . . . . . . . . 37

4 Transport in two-channel setups $\quad 39$

4.1 Transport in the Goldhaber-Gordon setup . . . . . . . . . . . 39

4.2 Transport in the charge two-channel Kondo setup: methods and key quantities . . . . . . . . . . . . . . . 42

4.2.1 Potentials and current operators . . . . . . . . . . 43

4.2.2 Emery-Kivelson mapping of the current operators . . 44

4.2.3 Equilibrium propagators of the model . . . . . . . . . 47

4.2.4 Non-equilibrium techniques: Keldysh formalism . . . . 50

4.2 .5 Linear response theory . . . . . . . . . . . 53 
$\begin{array}{lll}5 & \text { Time-dependent charge transport at the exactly solvable point } & \mathbf{5 7}\end{array}$

5.1 Electrical current as a voltage-dependent integral . . . . . . . 57

5.2 The de solution . . . . . . . . . . . . . . . . . . 61

5.3 The ac solution . . . . . . . . . . . . . . . . . . 65

5.4 Relaxation to a new steady state $\ldots \ldots \ldots . \ldots 71$

$6 \quad$ Linear susceptibilities of the charge two-channel Kondo model 77

6.1 Charge transport . . . . . . . . . . . 77

6.2 Heat transport $\ldots \ldots \ldots \ldots \ldots$. . . . . . . . . . . . 82

7 Wiedemann-Franz law in a non-Fermi liquid and Majorana central charge 95

7.1 Wiedemann-Franz law at the non-Fermi liquid fixed point . . 95

7.2 Central charge of the critical system $\ldots \ldots \ldots \ldots 9$

7.3 Comparison with the Goldhaber-Gordon setup . . . . . . . . 98

7.4 Conclusion $\ldots \ldots \ldots \ldots \ldots$

8 Extensions 101

8.1 Heat transport away from the non-Fermi liquid fixed point. . 101

8.2 Beyond the universal Fermi liquid crossover . . . . . . . . 103

8.3 The one-dimensional two-lead Kondo lattice . . . . . . . . . . 107

8.4 Outlook . . . . . . . . . . . . . . . . . . . . 110

\begin{tabular}{ll}
\hline Appendix & 113
\end{tabular}

A.1 Bosonization . . . . . . . . . . . . . . . . . 113

A.2 Majorana Green functions on the dot. . . . . . . . . . . . 115

A.3 Notes on the Keldysh structure . . . . . . . . . . . . . . . . 118

A.4 Kubo formula . . . . . . . . . . . . . . . . . . . . . 120

A.5 Digamma function . . . . . . . . . . . . . . . . . . . 123

A.6 Bessel functions of the first kind . . . . . . . . . . . . . . 126

A.7 Ac current in absence of a magnetic field. . . . . . . . . . . 127

A.8 Vanishing bubble diagrams $\ldots \ldots \ldots \ldots$. . . . . . . . . . 128

A.9 Heat transport: supplementary material . . . . . . . . . . . . 131

A.10 Self-energy away from the Emery-Kivelson point . . . . . . . 137

$\begin{array}{ll}\text { Bibliography } & 141\end{array}$

\begin{tabular}{ll}
\hline Nederlandse samenvatting & 149
\end{tabular}

\begin{tabular}{ll}
\hline Acknowledgements & 155
\end{tabular}

\begin{tabular}{ll}
\hline About the author & 157
\end{tabular} 


\section{Preface}

This thesis is the culmination of four years of research in the field of theoretical condensed matter physics, performed at the Institute for Theoretical Physics in Utrecht. In addition to presenting the results of our research, I aimed for this thesis to be a comprehensive pedagogical overview of the relevant concepts, such as Kondo physics and strong electronic correlations. To keep the text as concise and comprehensible as possible, I decided against including the smaller unrelated side projects (about topology) that I have also been part of, instead only listing the corresponding publications.

The first three chapters of this thesis serve as an extended introduction of the subject and the related concepts, providing motivation for the research and thoroughly explaining what is being studied. Subsequently, Chapter 4 introduces the methods, and Chapters 5 and 6 dive deep into the technical details of our research. Chapter 7 then discusses the consequences of the results and the overall conclusions, while the final chapter contains an overview of possible extensions of our work. Since the main text assumes that the reader has an advanced understanding of theoretical physics, I have also provided a Dutch summary at the end of the thesis that is hopefully more accessible to a wider audience.

I hope that the reader will learn a thing or two from my thesis!

Gerwin van Dalum

May 2020, Utrecht 



\section{List of publications}

This thesis is based on the following articles:

- G. A. R. van Dalum, A. K. Mitchell, and L. Fritz, "Wiedemann-Franz law in a non-Fermi liquid and Majorana central charge: Thermoelectric transport in a two-channel Kondo system", Phys. Rev. B 102, 041111 (2020).

- G. A. R. van Dalum, A. K. Mitchell, and L. Fritz, "Electric and heat transport in a charge two-channel Kondo device", submitted to Phys. Rev. B, arXiv preprint arXiv:2007.07239 (2020).

Other publications to which the author has contributed:

- E. L. Minarelli, K. Pöyhönen, G. A. R. van Dalum, T. Ojanen, and L. Fritz, "Engineering of Chern insulators and circuits of topological edge states", Phys. Rev. B 99, 165413 (2019).

- G. A. R. van Dalum, C. Ortix, and L. Fritz, "Magnetic impurities along the edge of a quantum spin-Hall insulator: Realizing a onedimensional AIII insulator", submitted to Phys. Rev. B, arXiv preprint arXiv:2007.04966 (2020). 



\section{Chapter 1}

\section{Introduction}

Without a doubt one of the most important results of modern physics is the rise of quantum electronics, such as the semiconductor devices that are widely used in present day technology. The field that studies the materials necessary to make such devices is known as condensed matter physics. In the most general sense, this field attempts to explain the properties of matter. Some of the questions that condensed matter physics concerns itself with are: why do some materials conduct electricity, while others do not? And how about a material's thermal conductivity? In the case of a crystalline solid, such questions are answered by exploiting the symmetry of the highly ordered crystal lattice structure of its microscopic components (often the individual atoms). This has led to the theory of electronic band structure, which examines a material's allowed quantum states to derive the range and momentum dependence of the energy levels of the electrons, thereby explaining its electronic transport properties. A proper understanding of a material's band structure potentially enables a wide variety of practical applications. However, for deriving these band structures it is often insufficient to only consider the simplest single electron excitations. Instead, it may be required to take more complicated excitations into account as well.

The field of condensed matter physics is fundamentally all about the collective properties of a large number of atoms. Individually, the microscopic components of these large systems (i.e. the individual atomic nuclei and the electrons) are well understood by virtue of the Standard Model of particle physics. However, large numbers of particles can exhibit collective behaviour that does not exist on the individual level. Imagine for example a simple point mass, which on its own does not have any complicated properties. If we take a large lattice of such point masses and connect all neighbouring lattice sites with springs, something interesting happens: by suddenly pushing one of the point masses, we can now send a wave through the larger system. Crucially, this propagating wave does not exist on the level of individual disconnected components. The development of such new collective behaviour in systems with many interacting particles is referred 
to as emergence and plays an integral role in condensed matter physics. Following the rules of quantum mechanics, the typically wave-like collective excitations can be quantized. This means that they can be interpreted as particles; the particles associated with vibrations in a lattice are for example known as phonons. These emergent effective particles are not elementary, and it is therefore possible to observe exotic effective particles that do not appear in the Standard Model, such as magnetic monopoles [1] or Majorana fermions [2]. Through collective excitations, condensed matter physics is thus a rich playground for studying all kinds of particles.

In this work we study strongly interacting electronic systems, which can potentially support unconventional collective excitations. In particular, we focus on so-called Kondo models, characterized by local magnetic impurities that strongly interact with some larger system. Especially the original single impurity Kondo model is historically one of the paradigmatic models of strong correlation physics, and has greatly improved our understanding of the effects of non-perturbative coupling [3]. Our aim is to further advance this understanding by theoretically studying the transport properties of nanoelectronic quantum dot devices that exhibit more complicated twochannel Kondo physics. In doing so, we will pay special attention to heat transport as a way to probe the systems' properties.

\subsection{Interacting fermions}

The following chapters are all about strongly interacting electrons. In order to gain a better understanding of the physics that will be encountered in these chapters, we start with a general overview of the behaviour of interacting fermions, in particular discussing the basics of Fermi liquid theory and its breakdown. This section follows parts of Refs. [4, 5].

\subsubsection{Fermi liquid theory}

Before discussing the effects of interactions, let us first briefly consider the non-interacting case. For non-interacting fermions, the ground state $|\Omega\rangle$ of an arbitrary system can be written as

$$
|\Omega\rangle=\prod_{|\mathbf{k}| \leq k_{F}, \sigma} c_{\mathbf{k} \sigma}^{\dagger}|0\rangle,
$$

where $|0\rangle$ is the vacuum state, $c_{\mathbf{k} \sigma}^{\dagger}$ are the fundamental second-quantized fermionic creation operators, $\mathbf{k}$ is the momentum, and $\sigma$ is the spin; note that 

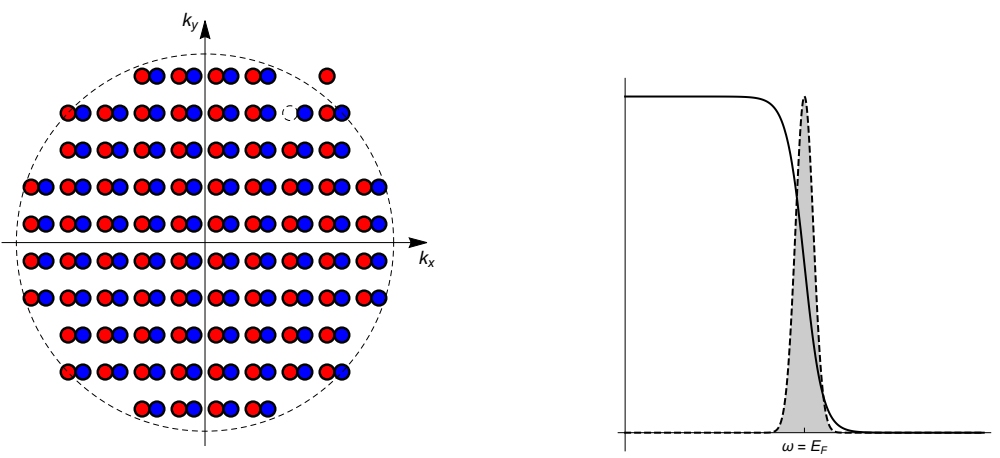

Figure 1.1: Left: ground state with a single particle-hole excitation of a noninteracting two-dimensional spin-1/2 fermion gas. The two particles of each pair represent the two spin states, while the large dashed circle denotes the Fermi momentum $k_{F}$ (i.e. the Fermi surface). Right: Fermi-Dirac distribution (solid) and a sketch of the excitation density (dashed).

the fermionic operators satisfy the anticommutation relations $\left\{c_{\mathbf{k} \sigma}, c_{\mathbf{k}^{\prime} \sigma^{\prime}}^{\dagger}\right\}=$ $\delta_{\mathbf{k}, \mathbf{k}^{\prime}} \delta_{\sigma, \sigma^{\prime}}$ and $\left\{c_{\mathbf{k} \sigma}, c_{\mathbf{k}^{\prime} \sigma^{\prime}}\right\}=\left\{c_{\mathbf{k} \sigma}^{\dagger}, c_{\mathbf{k}^{\prime} \sigma^{\prime}}^{\dagger}\right\}=0$. The expression from Eq. 1.1 means that all states up to the Fermi surface with momentum $k_{F}$ are occupied, while all other states are empty. At finite temperatures, some particles will instead occupy an excited state with $|\mathbf{k}|>k_{F}$ (or $\epsilon_{\mathbf{k}}>E_{F}$ in terms of energy) while leaving behind a hole with $|\mathbf{k}| \leq k_{F}$, see the left panel of Fig. 1.1. In operator language, the creation operators of these holes are identical to the annihilation operators of the particles, $c_{\mathbf{k} \sigma}$. The finite temperature particle density satisfies the Fermi-Dirac distribution,

$$
n(\mathbf{k})=\frac{1}{e^{\left(\epsilon_{\mathbf{k}}-E_{F}\right) / k_{B} T}+1},
$$

where $T$ is the temperature and $k_{B}$ the Boltzmann constant. Consequently, the excitation density, corresponding to the creation operators

$$
\psi_{\mathbf{k} \sigma}^{\dagger}= \begin{cases}c_{\mathbf{k} \sigma}^{\dagger}, & |\mathbf{k}|>k_{F}, \\ c_{\mathbf{k} \sigma}, & |\mathbf{k}| \leq k_{F},\end{cases}
$$

is peaked at the Fermi level $E_{F}$, while the width of this peak is proportional to the temperature $T$ (right panel of Fig. 1.1). The latter statement leads to the important consequence that the excitation density is sharply peaked in the limit $T \rightarrow 0$. This property dictates the qualitative low-temperature behaviour of several observables, such as the heat capacity, $C_{V} \propto T$ [6]. 
In the presence of interactions the particles can also exchange energy, which modifies the distribution function. If the interactions are weak, however, the ground state of the system is only weakly modified. The occupied states are slightly "moved around" in momentum space, such that weak interactions have a similar effect on the distribution function as the presence of a low-temperature thermal bath. Naively extrapolating this picture to strong interactions, one would expect the distribution function to be significantly broadened by the presence of the interactions. As a result, the lowtemperature excitation density would no longer be sharply peaked, which would for example change $C_{V}$ to no longer be linear in $T$ at low temperatures. It turns out that this is actually not true in many cases: even systems with strong interactions can often adequately be described as if they are weakly interacting, at least qualitatively and in the limit of low temperatures. These systems are called Fermi liquids, the theory of which was first developed by Landau in 1956 [7, 8].

To understand why Fermi liquids behave as if they are weakly interacting, we consider the general interacting single-particle retarded Green function

$$
G_{\mathbf{k}}(\omega)=\frac{1}{\omega-\epsilon_{\mathbf{k}}+E_{F}-\Sigma_{\mathbf{k}}(\omega)+i 0^{+}},
$$

where $\Sigma_{\mathbf{k}}(\omega)$ is the self-energy. For non-interacting systems the self-energy is zero, such that the corresponding spectral function (i.e. the density of states as a function of momentum $\mathbf{k}$ and energy $\omega$ ) reduces to

$$
A(\mathbf{k}, \omega)=-\frac{1}{\pi} \operatorname{Im}\left[G_{\mathbf{k}}(\omega)\right] \rightarrow \delta\left(\omega-\epsilon_{\mathbf{k}}+E_{F}\right) .
$$

The density of states is therefore infinitely sharply peaked at $\omega=\epsilon_{\mathbf{k}}-E_{F} \equiv$ $E_{\mathbf{k}}$, which translates to well-defined particles with definite momentum $\mathbf{k}$. Moreover, using the dispersion $\epsilon_{\mathbf{k}}=\hbar^{2} \mathbf{k}^{2} / 2 m$, linearization of the energy around $\mathbf{k}=k_{F} \hat{\mathbf{x}}$ gives $E_{\mathbf{k}}=\hbar^{2} k_{F}\left(k_{x}-k_{F}\right) / m+\ldots$, showing that the derivative of the energy close to the Fermi momentum depends solely on the mass of the particle and the Fermi momentum. In the more general non-interacting case, the spectral function can be written as

$$
A(\mathbf{k}, \omega)=-\frac{1}{\pi} \frac{\operatorname{Im}\left[\Sigma_{\mathbf{k}}(\omega)\right]}{\left(\omega-\epsilon_{\mathbf{k}}+E_{F}-\operatorname{Re}\left[\Sigma_{\mathbf{k}}(\omega)\right]\right)^{2}+\left(\operatorname{Im}\left[\Sigma_{\mathbf{k}}(\omega)\right]\right)^{2}} .
$$

The spectral function is therefore still peaked, however the peak now has a finite width (proportional to $-\operatorname{Im}\left[\Sigma_{\mathbf{k}}(\omega)\right]>0$ ), and the location of the peak 
is modified. Now assume that the imaginary part of the self-energy vanishes. Then, the spectral function once again reduces to a Dirac delta function, but located at the solution of the equation

$$
\omega-\epsilon_{\mathbf{k}}+E_{F}-\operatorname{Re}\left[\Sigma_{\mathbf{k}}(\omega)\right]=0 .
$$

The solution of this equation, $\omega=E_{\mathbf{k}}^{*}$, is in general very different from the non-interacting solution $E_{\mathbf{k}}=\epsilon_{\mathbf{k}}-E_{F}$. However, once the solution has been found, it can again be linearized. Comparing this linearization to its noninteracting counterpart, we find an effective mass $m^{*}$. Moreover, we can rewrite the spectral function as

$$
A(\mathbf{k}, \omega)=\delta\left(\omega-\epsilon_{\mathbf{k}}+E_{F}-\operatorname{Re}\left[\Sigma_{\mathbf{k}}(\omega)\right]\right)=\mathcal{N}_{\mathbf{k}} \delta\left(\omega-E_{\mathbf{k}}^{*}\right),
$$

showing that the spectral function with fixed momentum $\mathbf{k}$ no longer integrates to 1 , but instead has a non-trivial weight. Instead of the original fundamental particles, we are thus dealing with collective excitations called quasiparticles with an effective mass $m^{*}$ and a quasiparticle weight $\mathcal{N}_{\mathbf{k}}$.

In addition to introducing an effective mass and a quasiparticle weight, the peak in the spectral function is also broadened. Since the time-dependent wave function corresponding to a well-defined energy $E$ is proportional to $e^{-i E t / \hbar}$ (i.e. it is oscillating with frequency $E / \hbar$ ), the presence of a complex self-energy introduces a damping factor $e^{-t / \tau}$, with

$$
\tau=-\frac{\hbar}{\operatorname{Im}\left[\Sigma_{\mathbf{k}}(\omega)\right]} .
$$

The broadening of the peak in the spectral function therefore gives the quasiparticles a finite lifetime $\tau$. Now in order for a quasiparticle to retain a welldefined energy, the frequency of the oscillations must be much larger than the inverse time scale of the damping: $E / \hbar \gg 1 / \tau$. The left-hand side of this inequality is simply the frequency $\omega$. Meanwhile, the right-hand side is proportional to the transition rate of a particle starting below the Fermi surface and ending up above it. Fermi's golden rule states that this transition rate is in turn proportional to the sum over all available initial and final states, which can be used to show that the transition rate is proportional to $\omega^{2}$. This quadratic dependence is rather atypical, and is a consequence of processes being suppressed by Pauli's exclusion principle. When approaching the Fermi level (i.e. when decreasing $\omega$ ), the condition $E / \hbar \gg 1 / \tau$ therefore tells us that the quasiparticles become increasingly well-defined, such that they are appropriate objects for investigating the physics of the interacting system close to the Fermi surface. 
The above is a rather technical explanation of how quasiparticles appear and why they can be used to describe interacting systems close to the Fermi surface. However, there is also an intuitive picture. First, we note that the ground state of an interacting system is in general very different from the ground state of a non-interacting system. Although the interacting ground state itself is typically unknown, we can often still study the low-lying excitations about this ground state by imagining that we adiabatically switch on the interactions. During this process the quantum numbers of the excitations (which started out as elementary particle-hole excitations) remain unchanged. There are now two types of interactions to consider: interactions between an excited particle and non-excited particles, and interactions between different excitations. At low temperatures, there are only a few excitations. As such, the excitations are located far apart, and the contribution of interactions between them is small. On the other hand, the interactions between an excitation and the surrounding non-excited particles remains strong. We therefore consider an excitation and its surroundings as a single entity which has the same quantum numbers as the fundamental particle, but with a larger mass and a different spectral weight. This collective excitation is the quasiparticle that we encountered before, and the interactions between different quasiparticles is by construction weak at low temperatures. Moreover, at low temperatures all quasiparticles are close to the (heavily modified) Fermi surface, such that Pauli blocking guarantees that they are long-lived and therefore appropriate for describing the lowtemperature physics. Despite the fact that the interactions are allowed to be strong, the low-energy excitations are thus weakly interacting quasiparticles, such that the qualitative low-temperature behaviour of the system is identical to that of the weakly interacting limit.

Finally, we list some of the low-temperature properties of Fermi liquids:

- the heat capacity is linear in temperature: $C_{V} \propto T[6]$;

- the magnetic susceptibility and the compressibility are both independent of the temperature [7];

- the Wiedemann-Franz law is typically satisfied, meaning that the ratio of the heat conductivity $\kappa$ over $T$ times the electrical conductivity $\sigma$ goes to a constant value, $\lim _{T \rightarrow 0} \kappa / T \sigma=\pi^{2} k_{B}^{2} / 3 e^{2}$ [9, 10].

These are the properties that are often used to identify a Fermi liquid for practical purposes. 


\subsubsection{Non-Fermi liquid physics}

Fermi liquid theory applies to many materials, however, it is not able to explain the behaviour of all interacting fermionic systems. The breakdown of Fermi liquid theory can for example happen when the Fermi surface of an interacting system is not a smooth deformation of its non-interacting counterpart, i.e. when the ground state undergoes fundamental changes when the interactions are switched on. Fermionic systems that do not display the low-temperature properties of a Fermi liquid are referred to as non-Fermi liquids. Such systems are in general difficult to treat due to the invalidity of Landau's quasiparticle description; instead, strong interactions have to be treated explicitly and non-perturbative methods are required.

One example of non-Fermi liquid physics can be found in one-dimensional systems of interacting fermions. One-dimensional systems are different from their higher dimensional counterparts due to the fact that particles cannot simply pass by each other, severely limiting their freedom. Even the weakest interactions can no longer be neglected due to this inability of individual particles to avoid each other. This destroys the quasiparticle peak in the spectral function, such that Fermi liquid theory no longer holds, independent of the interaction strength. Another class of systems for which the Fermi liquid description tends to break down are frustrated systems. These are systems with competing interactions that have a complicated ground state due to the inability of the system to choose between its competing interactions. In particular, the term "frustrated" refers to the fact that the individual components of the Hamiltonian cannot simultaneously be minimized, typically leading to a highly degenerate ground state ${ }^{1}$ The frustrated nature of the system fundamentally changes the nature of the low-lying excitations, leading to qualitatively different behaviour that is not accounted for by Fermi liquid theory. This type of non-Fermi liquid physics will play an important role in later chapters.

\subsection{Outline}

In this thesis we investigate the transport properties of so-called two-channel Kondo setups that exhibit non-Fermi liquid behaviour due to frustration,

\footnotetext{
${ }^{1}$ An example of frustration is found in a set of three antiferromagnetically coupled Ising spins on a triangle: after the first two spins are chosen to anti-align, the third spin cannot simultaneously anti-align with both other spins. Due to the symmetry of the system, the third spin is unable to choose which bond to satisfy. The ground state is six-fold degenerate, while there are only eight possible states in total.
} 
most importantly the setup that was recently realized experimentally in Ref. [11. The motivation to study this particular setup over other similar setups is the unprecedented control to probe its non-Fermi liquid critical fixed point, such that our theoretical predictions concerning the non-Fermi liquid physics of this setup are within reach of existing experiments. With that in mind, this thesis is structured as follows. In Chapters 2 and 3 , we first introduce the original Kondo model and its properties, which we follow up with an elaborate discussion of the more complicated two-channel Kondo model. These chapters are thus entirely based on previous work, see the corresponding references. In Chapter 4, we subsequently adapt several known general methods for calculating transport properties to two-channel Kondo setups, forming the foundation for the later chapters. In Chapter 5 , we apply these methods to thoroughly investigate time-dependent charge transport at the exactly solvable point of the two-channel Kondo model, and by extension in the experimentally relevant setup. This chapter's calculations are inspired by previous work on a closely related model [12, 13] and lead to new results for the time-dependent electrical current due to different voltage profiles. We then continue by calculating the linear response heat conductance at the non-Fermi liquid critical point in Chapter 6, after which we discuss the physical implications and consequences of our results in Chapter 7. In particular, we discuss both the conservation of the Wiedemann-Franz law and the relationship of the heat conductance with the excitations' Majorana nature at the non-Fermi liquid critical point. The successful calculation of the heat conductance of a non-Fermi liquid and the implications that the result carries for the broader understanding of strong correlation physics are the most important outcomes of this thesis. Finally, in Chapter 8, we provide an overview of possible extensions of our research. The interested reader is furthermore encouraged to consult the elaborate appendix whenever more details are desired. 


\section{Chapter 2}

\section{The Kondo effect}

In this chapter we will consider the most important aspects of the Kondo model as it was originally proposed by Kondo in 1964 [14], necessary to understand the physics of the more complicated setups of the later chapters. The original Kondo model describes a system consisting of a sea of non-interacting itinerant electrons and a single localized magnetic spin- $1 / 2$ impurity that is antiferromagnetically coupled to the bath. The Hamiltonian of the Kondo model is given by

$$
\hat{H}_{K}=\sum_{\mathbf{k}, \sigma} \epsilon_{\mathbf{k}} \psi_{\mathbf{k} \sigma}^{\dagger} \psi_{\mathbf{k} \sigma}+\frac{J_{\perp}}{2}\left(s^{+} \tau^{-}+s^{-} \tau^{+}\right)+J_{z} s^{z} \tau^{z},
$$

with

$$
\mathbf{s} \equiv \frac{1}{2} \sum_{\sigma, \sigma^{\prime}} \psi_{\sigma}^{\dagger}(0) \boldsymbol{\sigma}_{\sigma \sigma^{\prime}} \psi_{\sigma^{\prime}}(0),
$$

where $\psi_{\mathbf{k} \sigma}$ represents the conduction electrons with momentum $\mathbf{k}$, energy $\epsilon_{\mathbf{k}}$ and spin $\sigma=\uparrow, \downarrow$; the field $\psi_{\sigma}(\mathbf{x})$ is the inverse Fourier transform of $\psi_{\mathbf{k} \sigma}$ to position space, using the convention

$$
\psi_{\mathbf{k} \sigma}=\frac{1}{\sqrt{N}} \sum_{\mathbf{x}} \psi_{\sigma}(\mathbf{x}) e^{-i \mathbf{k} \cdot \mathbf{x}} \quad \Longleftrightarrow \quad \psi_{\sigma}(\mathbf{x})=\frac{1}{\sqrt{N}} \sum_{\mathbf{k}} \psi_{\mathbf{k} \sigma} e^{i \mathbf{k} \cdot \mathbf{x}} .
$$

In the above expressions, $\boldsymbol{\tau}$ is the impurity spin operator, $\mathbf{s}$ is the spin of the bath at the impurity site (defined as the origin), $\boldsymbol{\sigma}$ is the vector of Pauli matrices, $J_{\perp}$ and $J_{z}$ are the components of the antiferromagnetic coupling constant, and $N$ is the number of lattice sites of the bath. Note that the spinflip term involving the combinations $s^{ \pm} \equiv s^{x} \pm i s^{y}$ and $\tau^{ \pm}$is allowed to have a different coupling constant than the Ising-like final term; if $J_{\perp}=J_{z} \equiv J$, these terms can be combined to the simplified form $J_{\mathbf{s}} \cdot \boldsymbol{\tau}$. As we shall see later, the above model gives rise to the Kondo effect: the impurity spin is asymptotically free at high temperatures, but becomes strongly bound into a non-trivial many-body singlet state at low temperatures [15]. Crucially, the strong coupling regime cannot be treated perturbatively. 


\subsection{Microscopic origin of the Kondo model}

Before diving deeper into the physics of the Kondo model, it is instructive to take a step back and take a closer look at the physical origin of the model. To that end, we also consider a different model, namely the Anderson impurity model [16]. This model, proposed by Anderson in 1961, is essentially a generalization of the above: it still describes a bath of non-interacting itinerant electrons coupled to a single localized impurity, but the latter is now assumed to be a site that can at most contain two spin- $1 / 2$ fermions by Pauli's exclusion principle. The impurity site can therefore either be empty, singly occupied, or doubly occupied. In addition, the Coulomb repulsion of the electrons occupying the impurity site is included by introducing an interaction term that penalizes the doubly occupied state. Explicitly, the Hamiltonian of the Anderson impurity model is given by

$$
\hat{H}_{A}=\sum_{\mathbf{k}, \sigma}\left[\epsilon_{\mathbf{k}} \psi_{\mathbf{k} \sigma}^{\dagger} \psi_{\mathbf{k} \sigma}+\left(V_{\mathbf{k}} d_{\sigma}^{\dagger} \psi_{\mathbf{k} \sigma}+h . c .\right)\right]+\epsilon_{d} \sum_{\sigma} n_{d \sigma}+U n_{d \uparrow} n_{d \downarrow},
$$

where $d_{\sigma}$ denotes the electrons on the impurity site, $n_{d \sigma} \equiv d_{\sigma}^{\dagger} d_{\sigma}$ is the corresponding number operator, and $\epsilon_{d}$ and $U>0$ are respectively the onsite potential and the Coulomb repulsion acting on that site. Electrons are allowed to enter and exit the impurity site by the hybridization parameter $V_{\mathbf{k}}$, representing the overlap between the wave functions of the itinerant electrons and the bound states of the impurity potential. In many ways, the Anderson impurity model is the simplest model to describe impurities in a metal: it simply assumes the metal to be a lattice with some generic unspecified dispersion relation, with the addition of a single localized bound state that can have different occupation levels. This description is even valid for metals with several such impurities, given that the impurities are sufficiently dilute ${ }^{\top}$

Let us now consider the case in which the parameters satisfy $\epsilon_{d}<0$ and $U+\epsilon_{d}>0$, meaning that the on-site potential is attractive and that the Coulomb repulsion is stronger than this attractive potential. The singly occupied states $|\uparrow\rangle,|\downarrow\rangle$ correspond to an impurity energy of $\epsilon_{d}$, while the

\footnotetext{
${ }^{1}$ The condition of the impurities being "sufficiently dilute" can be made more quantitative by analysing the propagators of the bare model (i.e. a metal without any impurities). In particular, these propagators provide a natural length scale, called the correlation length, beyond which all correlations become negligible. If the typical distance between neighbouring impurities is much larger than this correlation length, then the impurities are independent of each other, such that the full system can adequately be approximated as a set of copies of a system with only a single impurity.
} 


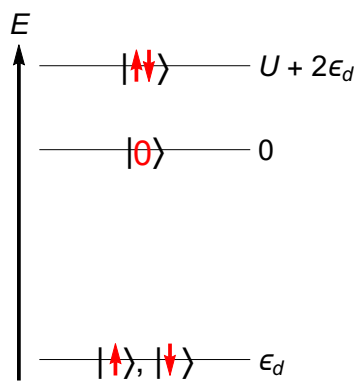

Figure 2.1: Illustration of the energies of the four possible impurity occupation states. The singly occupied states correspond to the lowest energy, such that they dominate the system's behaviour at low temperatures. In this particular example, we chose $U+\epsilon_{d}>-\epsilon_{d}$.

empty and doubly occupied states $|0\rangle$ and $|\uparrow \downarrow\rangle$ contribute an energy of 0 and $U+2 \epsilon_{d}$, respectively. The singly occupied states are therefore energetically favourable, see Fig. 2.1, and the low-energy dynamics of the model are dominated by excitations about these states, i.e. processes in which a singly occupied state must always return to a singly occupied state shortly after being excited. In the low-energy regime, the impurity can thus be approximated as a spin- $1 / 2$ degree of freedom that can only change (flip) by means of virtual intermediate states involving the bath. This is exactly the situation that the Kondo model from Eq. (2.1) aims to describe, explaining its microscopic origin.

In addition to this intuitive picture, the relation between the Anderson and Kondo models can be proven explicitly by means of a Schrieffer-Wolff transformation [3, 17, outlined below for later reference. The idea of this transformation is to derive an effective Hamiltonian by projecting the system onto the singly occupied states. This method requires first defining projection operators $\hat{P}_{i}$ that isolate the components of the wave function involving $i$ electrons on the impurity site. The appropriate projection operators corresponding to these three subspaces are given by

$$
\begin{aligned}
& \hat{P}_{0}=\left(1-n_{d \uparrow}\right)\left(1-n_{d \downarrow}\right), \\
& \hat{P}_{1}=n_{d \uparrow}\left(1-n_{d \downarrow}\right)+n_{d \downarrow}\left(1-n_{d \uparrow}\right), \\
& \hat{P}_{2}=n_{d \uparrow} n_{d \downarrow} .
\end{aligned}
$$

Letting the projectors $\hat{P}_{i}$ act on the Schrödinger equation $\hat{H}|\psi\rangle=E|\psi\rangle$ from the left, we find three independent components of the Schrödinger equation 
involving the three components of the wave function $\left|\psi_{i}\right\rangle \equiv \hat{P}_{i}|\psi\rangle$ :

$$
\sum_{j} \hat{H}_{i j}\left|\psi_{j}\right\rangle=E\left|\psi_{i}\right\rangle
$$

Here, $\hat{H}_{i j} \equiv \hat{P}_{i} \hat{H} \hat{P}_{j}$ are the components of the Hamiltonian that bring the occupancy of the impurity from $j$ to $i$, satisfying $\hat{H}_{j i}=\left(\hat{H}_{i j}\right)^{\dagger}$. These components can straightforwardly be deduced from Eq. (2.4) together with the above projection operators, for example

$$
\hat{H}_{11}=\hat{P}_{1}\left[\sum_{\mathbf{k}, \sigma} \epsilon_{\mathbf{k}} \psi_{\mathbf{k} \sigma}^{\dagger} \psi_{\mathbf{k} \sigma}+\epsilon_{d}\right], \quad \hat{H}_{12}=\sum_{\mathbf{k}, \sigma} V_{\mathbf{k}} \psi_{\mathbf{k} \sigma}^{\dagger} n_{d \bar{\sigma}} d_{\sigma},
$$

where the notation $\bar{\sigma}$ indicates the opposite spin of $\sigma$. It is especially helpful to note that $\hat{H}_{02}=\hat{H}_{20}=0$, which is due to the fact that Eq. 2.4 does not involve any processes transporting two electrons simultaneously. The effective Hamiltonian $\hat{H}_{\text {eff }}$ for the singly occupied states can now be found by realizing that it must satisfy its own Schrödinger equation, $\hat{H}_{\text {eff }}\left|\psi_{1}\right\rangle=E\left|\psi_{1}\right\rangle$. Using the $i=0$ and $i=2$ components from Eq. (2.8) to eliminate $\left|\psi_{0}\right\rangle$ and $\left|\psi_{2}\right\rangle$ from the $i=1$ equation, we can identify

$$
\hat{H}_{\text {eff }}=\hat{H}_{11}+\hat{H}_{12}\left(E \mathbb{1}-\hat{H}_{22}\right)^{-1} \hat{H}_{21}+\hat{H}_{10}\left(E \mathbb{1}-\hat{H}_{00}\right)^{-1} \hat{H}_{01},
$$

where $\mathbb{1}$ is the identity matrix. The three terms of this effective Hamiltonian correspond to three types of processes involving the singly occupied states: (i) processes that do not change the occupancy of the impurity site; (ii) processes that start with an additional electron hopping onto the impurity site and end with an electron leaving it, while the occupancy remains unchanged during the intermediate dynamics; (iii) processes that start with the electron hopping off the impurity site and end with an electron entering it, again without any intermediate changes to the occupancy. In the lowenergy limit of the case in which the singly occupied states are energetically favourable, these are the only remaining processes, further establishing the relation between the two models. Finally, the explicit connection can now be derived by plugging the components $\hat{H}_{i j}$ into Eq. 2.10 and applying the appropriate anticommutation relations. For a localized hybridization $V_{\mathbf{k}} \equiv V / \sqrt{N}$ and in terms of spin operators defined according to Eq. 2.2 and $\boldsymbol{\tau} \equiv \sum_{\sigma, \sigma^{\prime}} d_{\sigma}^{\dagger} \sigma_{\sigma \sigma^{\prime}} d_{\sigma^{\prime}} / 2$, the resulting effective Hamiltonian (up to a scattering term that reduces to a simple single-particle energy) is given by

$$
\hat{H}_{A, \mathrm{eff}}=\sum_{\mathbf{k}, \sigma} \epsilon_{\mathbf{k}} \psi_{\mathbf{k} \sigma}^{\dagger} \psi_{\mathbf{k} \sigma}+J \mathbf{s} \cdot \boldsymbol{\tau}+\mathcal{O}\left(V^{4} / \epsilon_{d}^{2}, V^{4} / U^{2}\right),
$$


with

$$
J=-\frac{2|V|^{2} U}{\epsilon_{d}\left(U+\epsilon_{d}\right)}>0 .
$$

Thus, the low-energy physics emerging from the Anderson impurity model for large on-site energy $\epsilon_{d}$ and interaction $U$ are identical to that of the isotropic Kondo model with antiferromagnetic coupling. The Kondo model is therefore indeed appropriate for investigating the low-temperature dynamics of a metal with dilute point-like impurities, where the Schrieffer-Wolff transformation dictates that the coupling constant $J$ must be strictly positive, i.e. antiferromagnetic.

With the above in mind, for the remainder of this chapter we will focus on the properties of the anisotropic Kondo model from Eq. (2.1). We will first review the resistance minimum that originally motivated Kondo to develop this model, and the so-called "Kondo problem" it introduces as $T \rightarrow 0$. Following that, we will discuss the concepts of strong correlations and the renormalization group that can be used to resolve these issues and understand the physics of this limit.

\subsection{Resistance minimum and the Kondo problem}

Typically, the resistivity of a metal decreases monotonically when lowering the temperature: it is phenomenologically given by

$$
R(T)=R_{0}+a T^{2}+b T^{5},
$$

where the quadratic term is due to Fermi liquid-like behaviour (i.e. electronelectron scattering), and the $T^{5}$ term is due to phonon scattering. The scattering rates decrease with the temperature, resulting in a decreasing resistance. However, since the 1930s it has been known experimentally that the resistivity can actually exhibit a minimum at a certain low temperature [18, see Fig. 2.2. By closely looking at the available experimental data, Kondo concluded that the existence of this minimum is likely a single-impurity effect ${ }^{2}$ and that it must be a magnetic effect. This motivated him to neglect all interactions between different impurities and consider the Hamiltonian from Eq. 2.1). Starting from the Kondo Hamiltonian, he then proceeded to

\footnotetext{
${ }^{2}$ Although the resistance minimum is a single-impurity effect, a finite density of impurities is still required to make a contribution. After all, a single microscopic impurity in a macroscopic system cannot significantly affect the system's macroscopic quantities.
} 


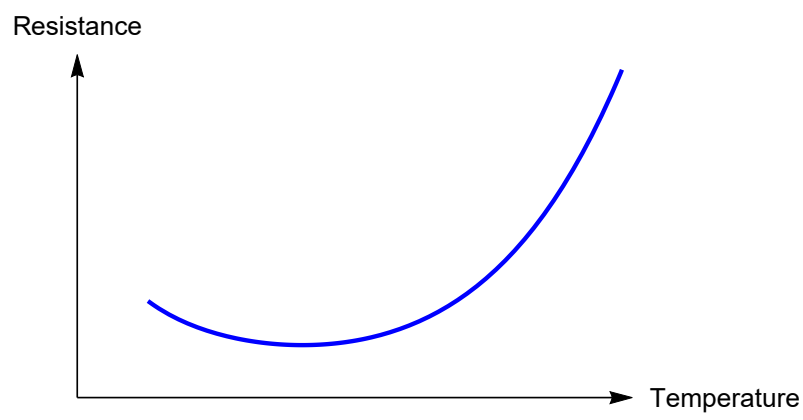

Figure 2.2: Sketch of the resistance of gold as a function of temperature, as it was first measured by De Haas, De Boer and Van den Berg in 1934 [18. The resistance profile shows a clear minimum at a certain low temperature.

perturbatively calculate the resistivity of the model by combining T-matrix formalism with the Drude formula. For the isotropic case and to leading order in $J$ (i.e. in the first Born approximation), this led to

$$
R(T)=R_{0}\left[1-2 \rho J \ln \left(\frac{k_{B} T}{\Lambda}\right)\right]+\mathcal{O}\left(J^{2}\right),
$$

see for example Ref. [3] for a detailed derivation. Here, $\rho$ is the density of states at the Fermi level, and $\Lambda$ the cut-off energy (commonly referred to as the bandwidth) of the conduction electrons. Reintroducing the $T^{2}$ and $T^{5}$ terms, the resistivity becomes

$$
R(T)=R_{0}+a T^{2}+b T^{5}-c \ln \left(\frac{k_{B} T}{\Lambda}\right)+\ldots,
$$

such that it indeed develops a minimum that is in excellent agreement with the experiments.

Although Kondo's perturbative approach correctly reproduces the resistance minimum, it also introduces another problem: according to the perturbative results, the resistivity diverges as the temperature goes to zero. However, when the temperature becomes sufficiently low, the correction term from Eq. 2.14 can no longer be small, such that the perturbative results are no longer valid. The suggestion that the resistivity diverges as $T \rightarrow 0$ is therefore premature. The temperature scale below which perturbation theory breaks down is called the Kondo temperature $T_{K}$, which is of the exponentially small order

$$
k_{B} T_{K} \sim \Lambda e^{-\frac{1}{\rho J}} .
$$


From the experiments, it is known that the resistance remains finite even for $T \ll T_{K}$. The next task is thus to find a proper theoretical description and a physical understanding of the system in the region $T \lesssim T_{K}$. This became known as the Kondo problem.

\subsection{Poor man's scaling and the renormalization group}

The solution to the Kondo problem lies in the concept of the renormalization group. An early version of this framework was developed by Anderson in 1970, who dubbed his technique "a poor man's derivation of scaling laws" [19]. We will now discuss this technique to some detail.

The main idea behind poor man's scaling is to find an effective Hamiltonian describing the low-lying excitations by systematically eliminating the outermost shell containing the highest-lying modes. The procedure for finding the effective Hamiltonian in each intermediate step is identical to the procedure from Sec. 2.1, although the subspaces and the associated projection operators are different. In order to eliminate the highest-lying modes, one has to divide the momentum-resolved operators $\psi_{\mathbf{k} \sigma}$ into two groups: those corresponding to electrons or holes with energy $\left|\epsilon_{\mathbf{k}}\right| \leq \Lambda / b$, and those with energy $\Lambda / b<\left|\epsilon_{\mathbf{k}}\right| \leq \Lambda$, where $\Lambda$ is again the bandwidth, and $b>1$ determines the width of the shell, see Fig. 2.3. We use this division to define the following three subspaces: the subspace of wave functions with no components in the outer shell (subspace 1), the subspace of wave functions that contain at least one electron with $\epsilon_{\mathbf{k}}>\Lambda / b$ (subspace 2), and the subspace of the remaining wave functions (all containing at least one hole with $\epsilon_{\mathbf{k}}<-\Lambda / b$, subspace 0 ). For large $\Lambda$, the direct connection between subspace 0 and subspace 2 goes to zero, such that elimination of the highestlying modes from the Schrödinger equation leads to the familiar expression

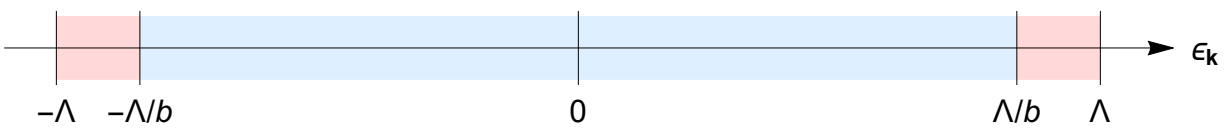

Figure 2.3: Sketch of the energy scales involved in the poor man's scaling procedure. During each iteration of the procedure, the modes residing in the outer shell with energy $\Lambda / b<\left|\epsilon_{\mathbf{k}}\right| \leq \Lambda$ are eliminated from the Schrödinger equation, such that the bandwidth is reduced from $\Lambda$ to $\Lambda / b$. The width of the outer shell $\Lambda(1-1 / b)$ is kept small with respect to the original bandwidth $\Lambda$. 
for the effective Hamiltonian acting on subspace 1,

$$
\hat{H}_{\text {eff }}=\hat{H}_{11}+\hat{H}_{12}\left(E \mathbb{1}-\hat{H}_{22}\right)^{-1} \hat{H}_{21}+\hat{H}_{10}\left(E \mathbb{1}-\hat{H}_{00}\right)^{-1} \hat{H}_{01} .
$$

Referring back to Eq. (2.1) and rescaling $J_{\perp}$ and $J_{z}$ by a factor $1 / N$ for conciseness, we can explicitly write down the components $\hat{H}_{i j}$, for example

$$
\begin{aligned}
\hat{H}_{11}=\hat{P}_{1}[ & \hat{H}_{0}+\frac{J_{\perp}}{2} \sum_{\mathbf{k}_{L}, \mathbf{k}_{L}^{\prime}}\left(\psi_{\mathbf{k}_{L} \uparrow}^{\dagger} \psi_{\mathbf{k}_{L}^{\prime} \downarrow} \tau^{-}+\psi_{\mathbf{k}_{L} \downarrow}^{\dagger} \psi_{\mathbf{k}_{L}^{\prime} \uparrow} \tau^{+}\right) \\
& \left.+\frac{J_{z}}{2} \sum_{\mathbf{k}_{L}, \mathbf{k}_{L}^{\prime}}\left(\psi_{\mathbf{k}_{L} \uparrow}^{\dagger} \psi_{\mathbf{k}_{L}^{\prime} \uparrow}-\psi_{\mathbf{k}_{L} \downarrow}^{\dagger} \psi_{\mathbf{k}_{L}^{\prime} \downarrow}\right) \tau^{z}\right], \\
\hat{H}_{12}=\hat{P}_{1}[ & \frac{J_{\perp}}{2} \sum_{\mathbf{k}_{L}, \mathbf{k}_{H}}\left(\psi_{\mathbf{k}_{L} \uparrow}^{\dagger} \psi_{\mathbf{k}_{H} \downarrow} \tau^{-}+\psi_{\mathbf{k}_{L} \downarrow}^{\dagger} \psi_{\mathbf{k}_{H} \uparrow} \tau^{+}\right) \\
+ & \left.\frac{J_{z}}{2} \sum_{\mathbf{k}_{L}, \mathbf{k}_{H}}\left(\psi_{\mathbf{k}_{L} \uparrow}^{\dagger} \psi_{\mathbf{k}_{H} \uparrow}-\psi_{\mathbf{k}_{L} \downarrow}^{\dagger} \psi_{\mathbf{k}_{H} \downarrow}\right) \tau^{z}\right],
\end{aligned}
$$

and $\hat{H}_{21}=\left(\hat{H}_{12}\right)^{\dagger}$, where $\hat{H}_{0}$ is the non-interacting kinetic part, $\mathbf{k}_{H}$ denotes the momenta corresponding to energies lying in the upper outer shell with $\epsilon_{\mathbf{k}_{H}}>\Lambda / b$, and $\mathbf{k}_{L}$ denotes all momenta corresponding to lower energies. Although the effective Hamiltonian does not yet have a particularly illuminating form, it should be noted that it is exact in the limit $\Lambda \rightarrow \infty$. To see how the desired information can be extracted, we will now consider the term that originates from taking the first term of Eq. 2.18) and its conjugate as an example. Acting on the the appropriate subspace, this term is given by the following expression:

$$
\frac{J_{\perp}^{2}}{4} \sum_{\mathbf{k}_{L}, \mathbf{k}_{L}^{\prime}, \mathbf{k}_{H}} \psi_{\mathbf{k}_{L} \uparrow}^{\dagger} \psi_{\mathbf{k}_{H} \downarrow} \tau^{-}\left(E \mathbb{1}-\hat{H}_{22}\right)^{-1} \psi_{\mathbf{k}_{H} \downarrow}^{\dagger} \psi_{\mathbf{k}_{L}^{\prime} \uparrow} \tau^{+} .
$$

Choosing the width of the outer shell to be small, the sum over $\mathbf{k}_{H}$ can be approximated as the density of states at the edge, $\rho$, times the width of the shell, $\Lambda(1-1 / b)$, while setting $\epsilon_{\mathbf{k}_{H}}=\Lambda$ in the summand. To leading order in $J_{\perp}$ and $J_{z}$, the term becomes

$$
\frac{J_{\perp}^{2} \rho \Lambda(1-1 / b)}{4} \sum_{\mathbf{k}_{L}, \mathbf{k}_{L}^{\prime}} \tau^{-} \tau^{+} \psi_{\mathbf{k}_{L} \uparrow}^{\dagger} \psi_{\mathbf{k}_{L}^{\prime} \uparrow} \frac{1}{E-\Lambda+\epsilon_{\mathbf{k}_{L}^{\prime}}},
$$

with $E$ being the energy measured with respect to the ground state energy. Finally noting that $\tau^{-} \tau^{+}=1 / 2-\tau^{z}$ and comparing the result to Eq. (2.17), 
we see that this additional term is of the same form as one of the previously existing terms, therefore acting as a renormalization of one of the parameters of the original Hamiltonian. In other words: this additional term in the effective Hamiltonian can be incorporated back into the original Hamiltonian by simply redefining the corresponding coupling constant. Repeating the above process for all other terms (see for example Ref. [3]), one finds that the full expressions for the renormalized coupling constants are given by

$$
\begin{aligned}
& J_{\perp}(b)=J_{\perp}-\frac{J_{\perp} J_{z} \rho \Lambda(1-1 / b)}{2}\left(\frac{1}{E-\Lambda+\epsilon_{\mathbf{k}}}+\frac{1}{E-\Lambda-\epsilon_{\mathbf{k}^{\prime}}}\right), \\
& J_{z}(b)=J_{z}-\frac{J_{\perp}^{2} \rho \Lambda(1-1 / b)}{2}\left(\frac{1}{E-\Lambda+\epsilon_{\mathbf{k}}}+\frac{1}{E-\Lambda-\epsilon_{\mathbf{k}^{\prime}}}\right)
\end{aligned}
$$

to leading order in $J_{\perp}$ and $J_{z}$. The effective Hamiltonian acting on subspace 1 is thus identical to the original Hamiltonian from Eq. (2.1), but with new renormalized coupling constants $J_{\perp}(b), J_{z}(b)$. When strictly considering the behaviour of the low-lying excitations, we furthermore have $E \ll \Lambda$ and $\epsilon_{\mathbf{k}} \ll \Lambda$, which simplifies these equations to

$$
J_{\perp}(b)=J_{\perp}+J_{\perp} J_{z} \rho(1-1 / b), \quad J_{z}(b)=J_{\perp}+J_{\perp}^{2} \rho(1-1 / b) .
$$

The above shows how the coupling constants are renormalized after a single scaling cycle. However, the goal of the poor man's scaling procedure is to find how the coupling constants are renormalized upon iteratively performing a series of these cycles until one reaches the desired energy scale, eliminating only an infinitesimal layer at a time. To this end, we define the parameter $l>0$ according to $b \equiv e^{l}$. Taking the limit of $l$ being infinitesimally small, we can write $(1-1 / b)=\mathrm{d} l$, such that

$$
\frac{\mathrm{d} J_{\perp}}{\mathrm{d} l}=J_{\perp} J_{z} \rho, \quad \frac{\mathrm{d} J_{z}}{\mathrm{~d} l}=J_{\perp}^{2} \rho .
$$

These equations are solved by the curves

$$
J_{z}^{2}-J_{\perp}^{2}=\text { const., }
$$

and the direction of the curves is found by noting that the derivative of $J_{z}$ is always positive. One particularly useful way of examining the behaviour dictated by the above equations is to draw the corresponding flow diagram, shown in Fig. 2.4. Given a set of initial parameters, this flow diagram tells us how the renormalized parameters of the effective model develop upon systematic elimination of the highest energetic modes. For the antiferromagnetic 


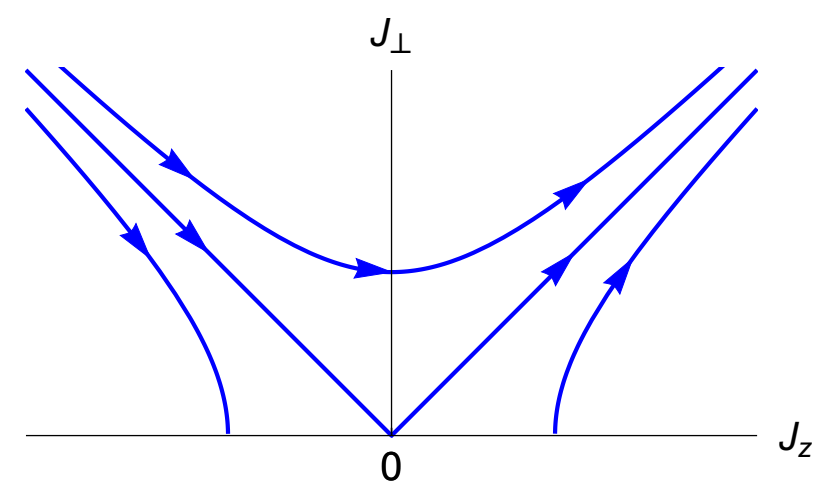

Figure 2.4: Scaling trajectories of the anisotropic Kondo model, as calculated using the poor man's scaling technique to lowest non-trivial order in the coupling constants $J_{\perp}$ and $J_{z}$.

model (i.e. $J_{\perp}>0$ and $J_{z}>0$ ), we thus see that the parameters eventually flow towards the infinitely strongly coupled isotropic point $J_{\perp}=J_{z} \rightarrow \infty$. This divergence is what causes the Kondo effect. Since the parameters flow towards isotropy, it is also insightful to solve the flow equations (2.24) for the isotropic case $J_{\perp}=J_{z} \equiv J$. The solution of the isotropic flow equation is given by

$$
J(l)=\frac{J}{1+\rho J \ln (\Lambda(l) / \Lambda)},
$$

where $J$ is the original (or "bare") coupling constant, $J(l)$ is the renormalized coupling constant, and $\Lambda(l) \equiv \Lambda / b=e^{-l} \Lambda$ is the "new" cut-off energy, which should be chosen of the order of the energy scale that is being studied (e.g. the thermal scale $k_{B} T$ ). From this expression, we see that there exists a cut-off energy scale $\Lambda\left(l^{*}\right)$ (defined as the Kondo energy scale) at which the effective coupling constant diverges:

$$
\Lambda\left(l^{*}\right) \equiv k_{B} T_{K}=\Lambda e^{-\frac{1}{\rho J}}=\Lambda(l) e^{-\frac{1}{\rho J(l)}} .
$$

This is exactly the Kondo energy scale that we previously encountered in Eq. 2.16). Moreover, the final equality of Eq. 2.27) demonstrates this quantity is scale invariant, as it is independent of $l$ and can therefore unambiguously be calculated at any point along the scaling trajectory. Using this final equality, we can also write

$$
\rho J(l)=\frac{1}{\ln \left(\Lambda(l) / k_{B} T_{K}\right)},
$$


such that the rescaled effective coupling constant is a universal function of the ratio of the cut-off energy $\Lambda(l)$ over the Kondo scale, independent of the original parameters of the model.

For future reference, let us briefly relate the above methods and results to the concept of the renormalization group. To do so, we first note that the poor man's scaling method is an example of one of the two steps that are usually performed in the more general renormalization group procedure. This approach, often formulated in the language of functional integrals (where each degree of freedom appears as an integration variable), consists of two steps. First, the high-momentum degrees of freedom are integrated out (equivalent to poor man's scaling), and second, the entire system is rescaled such that the bandwidth returns to its original value. In general, this procedure leads to several additional terms in the effective Hamiltonian, some of which can be absorbed by redefining the parameters of the existing terms, while the other terms remain separate and appear as completely new terms. If a parameter increases in size during this procedure, it is said to be relevant, and parameters that do not flow are called marginal. Meanwhile, a parameter that decreases in size and eventually flows to zero is called irrelevant, as it does not play any role in the effective low-energy model. In the case of the antiferromagnetic Kondo model, both coupling constants are therefore relevant parameters, while anisotropies $\Delta J_{z} \equiv J_{z}-J_{\perp}$ are irrelevant. On the other hand, for the ferromagnetic model with $J_{z}<0$ and $\left|J_{\perp}\right|=\left|J_{z}\right|$, both coupling constants are irrelevant. Finally, any possible newly generated terms are at least of the order $1 / \Lambda$, hence they are irrelevant as well, and the effective model preserves the form of the original Hamiltonian.

At this point, it is also important to stress that all of this section's derivations once again made use of lowest-order perturbation theory in the coupling constants. Combined with the observation that the coupling constants become large when $\Lambda(l)$ approaches $k_{B} T_{K}$, this means that the above analysis is not accurate for energy scales around or below the Kondo scale. As a result, the poor man's scaling approach does not solve the Kondo problem of finding an appropriate low-energy model that is also valid below the Kondo scale. However, as was suspected by Anderson and later confirmed by Wilson using his non-perturbative numerical renormalization group calculations [20], many of the conclusions drawn from poor man's scaling are still correct. In particular, the Kondo temperature is indeed a scaling invariant, and the renormalized coupling constants do indeed follow a universal function of $T / T_{K}$. Moreover, even though the coupling constants actually remain finite at the Kondo temperature, they do in fact diverge isotropically as $T / T_{K} \rightarrow 0$. The Kondo model therefore flows towards the so-called 
strong coupling fixed point as $T / T_{K} \rightarrow 0$, corresponding to an effectively infinitely strong coupling between the bath and the impurity. Meanwhile, the limit $T / T_{K} \rightarrow \infty$ (called the local moment fixed point) corresponds to weak coupling, due to the fact that the finite coupling becomes small with respect to large temperature scale $k_{B} T$. This is analogous to the concepts of confinement and asymptotic freedom from high energy physics [21, 22], essentially providing a condensed matter version of these phenomena.

\subsection{Physics of the strong coupling fixed point}

The statement that the Kondo model flows towards the isotropic strong coupling fixed point as $T / T_{K} \rightarrow 0$ suggests that the ground state of the Kondo model is identical to the ground state of the Hamiltonian $\hat{H}=J_{\mathbf{s}} \cdot \boldsymbol{\tau}$, since the kinetic term is infinitesimally small in the limit $J \rightarrow \infty$. For $J>0$, the ground state of the latter is simply a singlet state

$$
\frac{1}{\sqrt{2}}(|\uparrow \Downarrow\rangle-|\downarrow \uparrow\rangle)
$$

with the impurity spin $\boldsymbol{\tau}$ (represented by $\uparrow, \downarrow$ ) and the spin of the bath at the impurity site $\mathbf{s}$ (represented by $\Uparrow, \Downarrow$ ) pointing in opposite directions. In order to show that this singlet state is also the ground state of the full Kondo model, it is necessary to prove that it is stable with respect to small perturbations caused by the kinetic term. In 1974, Nozières did so by means of perturbations theory in $1 / J[15]$, confirming that the ground state of the Kondo model is indeed the above singlet state. Moreover, his analysis showed that the heat capacity and the magnetic susceptibility of the impurity in the limit $T / T_{K} \rightarrow 0$ are identical to those of a Fermi liquid, as previously discussed in Sec. 1.1.1. For this reason, we identify the effective low-energy model as a local Fermi liquid.

Despite the fact that the interactions become strong below $T_{K}$, in many ways the system appears only weakly interacting at low temperatures. Physically, this is due to the impurity spin being screened by the conduction electrons in its vicinity: the singlet state that is formed at the impurity site is non-magnetic and therefore essentially invisible to the other conduction electrons. These itinerant electrons do not directly feel the presence of the impurity spin anymore, removing any divergences that would otherwise be caused by infinitely strong magnetic interactions. Instead, the singlet state acts as a non-magnetic infinitely repulsive impurity, leading to more conventional simple impurity scattering. With this in mind, the physical picture 


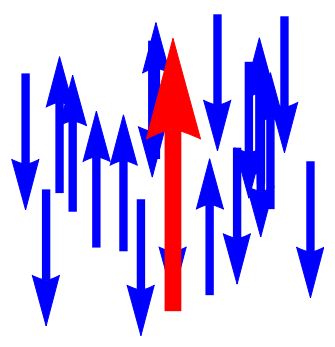

$T \gg T_{K}$

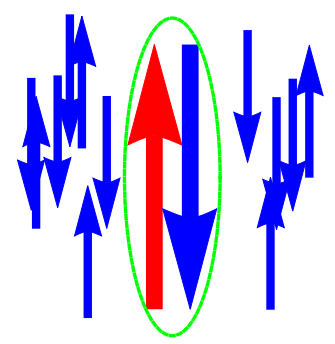

$T \ll T_{K}$

Figure 2.5: Illustration of the two limits of the Kondo model. Left: local moment fixed point, at which the impurity spin acts as an isolated local moment that only weakly interacts with the itinerant electrons. Right: strong coupling fixed point, characterized by the formation of a non-trivial singlet state between the impurity spin and the surrounding conduction electrons. This singlet state is non-magnetic, such that the impurity spin is screened from the rest of the bath.

behind the resistance minimum discussed in Sec. 2.2 finally becomes clear: as the temperature falls below $T_{K}$ (i.e. the effective coupling constant becomes large), the conduction electrons passing by the impurity spin develop the tendency to stay at the impurity site and form a singlet state. This effectively causes drag for the itinerant electrons, such that the resistance increases with the coupling constant. However, this effect does not extent all they way to zero temperature, since the singlet state becomes increasingly locked in. As such, the electrons gradually stop feeling the strong antiferromagnetic coupling, and the resistance saturates.

To conclude our discussion of the original Kondo model, let us summarize the most important aspects of its behaviour at different temperatures, illustrated in Fig. 2.5. First, at high temperatures the effective coupling is weak, such that the impurity spin acts as a more or less isolated local moment. On the other hand, for $T \lesssim T_{K}$ the effective coupling is strong, causing the formation of a non-magnetic singlet state of the impurity spin and its surrounding conduction electrons. Finally, as $T / T_{K} \rightarrow 0$ this screening of the impurity spin is complete, and the system becomes a Fermi liquid. 



\section{Chapter 3}

\section{The two-channel Kondo model}

The Kondo model has played a prominent role in the development of methods for studying strongly correlated materials, both on an experimental and a theoretical level [20, 23 26]. However, the Kondo model as it was described in the previous chapter still displays the properties of a relatively simple Fermi liquid (FL). A natural extension of the original (or "one-channel") Kondo model is the multichannel Kondo model [27]. In this model there are several independent baths, each with its own transport channel and all coupled to the same magnetic Kondo impurity. The ground states of multichannel Kondo systems can exhibit exotic non-Fermi liquid (NFL) behaviour due to frustrated screening of the impurity spin [28, 29, making the multichannel Kondo model a particularly interesting model to analyse. This chapter serves as a rigorous introduction to the two-channel Kondo (2CK) model, covering the model itself, its experimental realizations, the physics it leads to, its exactly solvable point, and how the latter can be used to study experimentally accessible observables.

\subsection{Model Hamiltonian and its ground states}

We start by introducing the most general anisotropic 2CK model, shown in Fig. 3.1 [13, 27]. The model consists of two baths (or "leads") and a local part, such that the Hamiltonian describing this model has the form $\hat{H}=\hat{H}_{\text {leads }}+\hat{H}_{\text {loc }}$. First, let us specify the leads. We model the threedimensional leads as effectively one-dimensional left-moving channels with Fermi velocity $v_{F}$ and a constant density of states [28, 30. This reduction from three-dimensional leads to a description in terms of one-dimensional left-movers is derived from the fact that the impurity is assumed to be a quantum dot that acts as a structureless point-like scatterer. The resulting rotational symmetry ensures that only simple s-wave scattering participates in the Kondo interaction, such that only the radial part of the wave function plays a role. The operators corresponding to the incoming and outgoing 


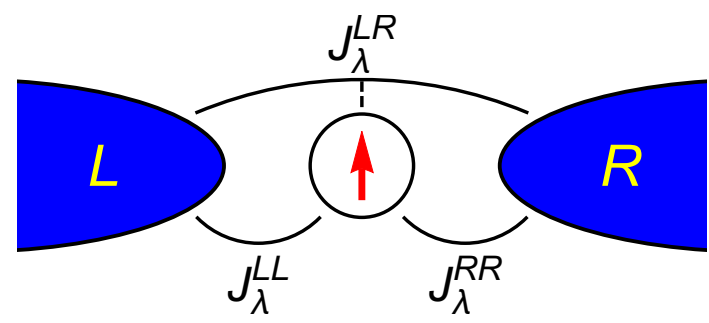

Figure 3.1: Schematic of the most general anisotropic 2CK model. The coupling constants $J_{\lambda}^{\alpha \beta}$ (where $\lambda=x, y, z$ ) are kept as general as possible, also allowing for impurity-mediated cotunnelling between the leads via $J_{\lambda}^{L R}$.

parts, both defined for a radial coordinate $r \geq 0$, are combined on a single directed one-dimensional chain with coordinate $x$ going from $-\infty$ to $\infty$. In particular, the incoming left-moving field is simply mapped to $x \geq 0$, while the outgoing right-moving field for $x \geq 0$ is replaced by a left-moving field for $x \leq 0$. In absence of any bias between the leads, we therefore have

$$
\hat{H}_{\text {leads }}=i \hbar v_{F} \sum_{\alpha} \sum_{\sigma} \int_{-\infty}^{\infty} \mathrm{d} x \psi_{\alpha \sigma}^{\dagger}(x) \partial_{x} \psi_{\alpha \sigma}(x),
$$

where $\psi_{\alpha \sigma}(x)$ are the chiral fermionic operators corresponding to the leftmoving fields representing the s-wave projected leads, $\alpha=L, R$ denotes the left and right leads, and $\sigma=\uparrow, \downarrow$ is the spin.

Meanwhile, the local part of the anisotropic $2 \mathrm{CK}$ model is kept as general as possible, such that it is described by the Hamiltonian

$$
\hat{H}_{\mathrm{loc}}=\sum_{\alpha, \beta} \sum_{\lambda} J_{\lambda}^{\alpha \beta} s_{\alpha \beta}^{\lambda} \tau^{\lambda},
$$

with

$$
\mathbf{s}_{\alpha \beta} \equiv \frac{1}{2} \sum_{\sigma, \sigma^{\prime}} \psi_{\alpha \sigma}^{\dagger}(0) \boldsymbol{\sigma}_{\sigma \sigma^{\prime}} \psi_{\beta \sigma^{\prime}}(0)
$$

Here, $J_{\lambda}^{\alpha \beta}=J_{\lambda}^{\beta \alpha}$ are the real coupling constants, $\mathbf{s}_{\alpha \beta}$ is the local electron spin density of the leads evaluated at the origin, and $\lambda=x, y, z$. Moreover, $\tau$ again represents the impurity spin- $1 / 2$ degree of freedom (from now on also referred to as "the dot"), while $\boldsymbol{\sigma}_{\sigma \sigma^{\prime}}$ are still the matrix elements of the vector of Pauli matrices. The terms with $\alpha=\beta$ are essentially identical 
to the interaction terms of the one-channel Kondo model from Eq. (2.1), but made more general by allowing the coupling in the $x-y$ plane to be anisotropic as well; for the special case $J_{x}^{\alpha \alpha}=J_{y}^{\alpha \alpha} \equiv J_{\perp}^{\alpha \alpha}$, they are again divided into spin-flip and Ising-like terms,

$$
\sum_{\alpha}\left[\frac{J_{\perp}^{\alpha \alpha}}{2}\left(s_{\alpha \alpha}^{+} \tau^{-}+s_{\alpha \alpha}^{-} \tau^{+}\right)+J_{z}^{\alpha \alpha} s_{\alpha \alpha}^{z} \tau^{z}\right] .
$$

However, in addition to these usual Kondo couplings between the individual baths and the impurity spin, there is also a set of coupling constants $J_{\lambda}^{L R}$ that allows for impurity-mediated cotunnelling between the leads. The reason for including this term can again be found in the Schrieffer-Wolff transformation of an Anderson impurity model, this time with two separate leads. Following the methods from Sec. 2.1, the effective Hamiltonian for a singly occupied Anderson impurity coupled to two leads must include virtual processes in which an electron is transported from one lead to the other. These processes are similar to their single-lead counterparts (discussed in Sec. 2.1) and in particular involve the same number of electron hoppings. The probability densities corresponding to the one- and two-lead processes are therefore of the same order of magnitude, such that the two-lead processes are not negligible. Mapped to the spin language, the virtual two-lead processes involve a hybridized spin density $\mathbf{s}_{\alpha \beta}$ (where $\alpha \neq \beta$ ), resulting in the impurity-mediated cotunnelling term that appears in Eq. (3.2). Finally, we also introduce a local constant magnetic field $B$ and couple it to the dot. The full Hamiltonian of the anisotropic 2CK model at zero bias between the leads is thus given by

$$
\hat{H}=i \hbar v_{F} \sum_{\alpha} \sum_{\sigma} \int_{-\infty}^{\infty} \mathrm{d} x \psi_{\alpha \sigma}^{\dagger}(x) \partial_{x} \psi_{\alpha \sigma}(x)+\sum_{\alpha, \beta} \sum_{\lambda} J_{\lambda}^{\alpha \beta} s_{\alpha \beta}^{\lambda} \tau^{\lambda}-B \tau_{z} .
$$

As we will discuss in more detail in Sec. 3.4 , the $2 \mathrm{CK}$ model can have two different ground states, depending on the coupling constants and the magnetic field: either both baths compete to screen the impurity spin, leading to a frustrated two-channel ground state, or one of the channels decouples from the rest of the system, resulting in the one-channel strong coupling fixed point between the remaining channel and the dot that was previously discussed in Sec. 2.4. In absence of a magnetic field and impurity-mediated cotunnelling, these ground states respectively correspond to symmetric and asymmetric coupling (i.e. $J_{\lambda}^{L L}=J_{\lambda}^{R R}$ and $J_{\lambda}^{L L} \neq J_{\lambda}^{R R}$ ). Intuitively, this is due to the fact that the system has a strong desire to screen the impurity 
spin in some way. The system usually achieves this by selecting whichever bath is coupled more strongly to screen the impurity, but in the symmetric case it is unable to choose one of the baths over the other.

Since the Schrieffer-Wolff transformation of a two-lead Anderson model dictates that $J_{\lambda}^{L R} \neq 0$, we also consider the effects that this cotunnelling has on the ground state of the system. To do so, we first write the coupling constants in matrix form:

$$
\mathbf{J}_{\lambda} \equiv\left(\begin{array}{ll}
J_{\lambda}^{L L} & J_{\lambda}^{L R} \\
J_{\lambda}^{L R} & J_{\lambda}^{R R}
\end{array}\right) .
$$

The above is written in the basis of left and right channels, associated with the left and right leads. It can be diagonalized by means of a unitary transformation $U$, leading to

$$
U^{\dagger} \mathbf{J}_{\lambda} U=\left(\begin{array}{cc}
J_{\lambda}^{e} & 0 \\
0 & J_{\lambda}^{o}
\end{array}\right)
$$

where

$$
J_{\lambda}^{e / o}=\frac{1}{2}\left(J_{\lambda}^{L L}+J_{\lambda}^{R R} \pm \sqrt{\left(J_{\lambda}^{L L}-J_{\lambda}^{R R}\right)^{2}+4\left(J_{\lambda}^{L R}\right)^{2}}\right)
$$

are the coupling constants corresponding to the independent "even" and "odd" channels (i.e. linear combinations of the left and right channels) [31]. From here, it is immediately clear that the two independent channels are only coupled symmetrically if both $J_{\lambda}^{L L}=J_{\lambda}^{R R}$ and $J_{\lambda}^{L R}=0$, such that the presence of a non-zero $J_{\lambda}^{L R}$ always leads to the one-channel ground state. As a result, a Kondo setup of which the microscopic origin is a simple twolead Anderson model can never achieve the frustrated two-channel ground state. In order to truly realize two-channel physics, it is therefore necessary to construct a more complicated setup in which all cotunnelling is blocked.

\subsection{First experimental realization}

The first experimental realization of true two-channel physics was achieved by Goldhaber-Gordon et al. using a quantum dot device simulating a more complicated Anderson impurity model 3234 . The model that describes the system consists of an interacting Anderson impurity, as previously seen in Eq. (2.4), coupled to three leads. Two of the leads, called the "source" and the "drain", are non-interacting baths in the thermodynamic limit. On the other hand, the third lead (or "right" lead) is a large but finite interacting 


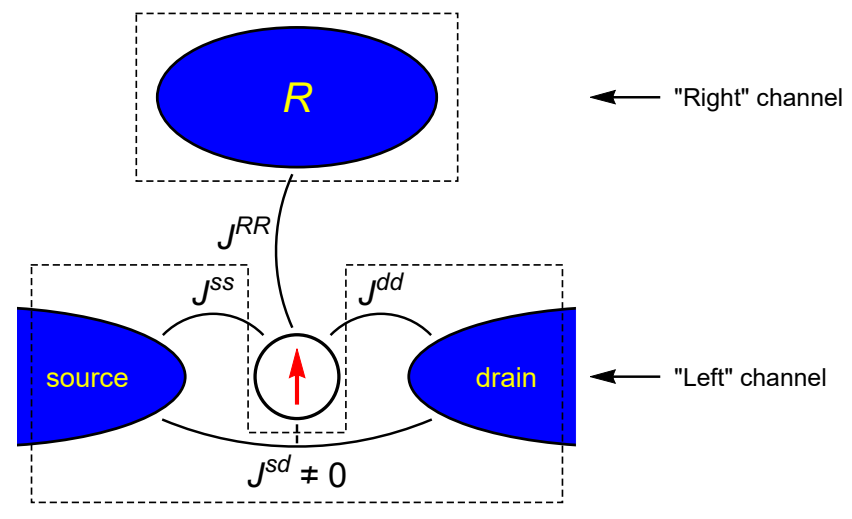

Figure 3.2: Schematic of Goldhaber-Gordon's experimental realization of the 2CK model 34. Lead $R$ is large but finite and has a charging energy that blocks all cotunnelling with the other leads, while the source and drain leads are non-interacting baths in the thermodynamic limit, such that they effectively form a single channel following the discussion from Sec. 3.1. The result is a $2 \mathrm{CK}$ model (the two channels being indicated by the dashed boxes) with $J^{L R}=0$, where $J^{R R}$ is simply the Kondo coupling of the finite interacting lead, and $J^{L L}$ is built up from the source/drain coupling constants $J^{s s}, J^{d d}$ and $J^{s d}$.

metallic island; in the low-energy regime, the interactions keep the number of electrons on this lead fixed. The Hamiltonian is thus given by

$$
\begin{aligned}
\hat{H}_{G-G}= & \sum_{\alpha} \sum_{\mathbf{k}, \sigma}\left[\epsilon_{\alpha \mathbf{k}} \psi_{\alpha \mathbf{k} \sigma}^{\dagger} \psi_{\alpha \mathbf{k} \sigma}+\left(V_{\alpha \mathbf{k}} d_{\sigma}^{\dagger} \psi_{\alpha \mathbf{k} \sigma}+\text { h.c. }\right)\right] \\
& +\epsilon_{d} \sum_{\sigma} n_{d \sigma}+U n_{d \uparrow} n_{d \downarrow}+\hat{H}_{R, \operatorname{int}}\left(\left\{\psi_{R \mathbf{k} \sigma}^{\dagger}\right\},\left\{\psi_{R \mathbf{k} \sigma}\right\}\right),
\end{aligned}
$$

where $\alpha=s, d, R$, and $\hat{H}_{R \text {,int }}$ governs the interactions on the right lead. Upon performing a Schrieffer-Wolff transformation (again following the procedure outlined in Sec. 2.1) and taking into account the interactions, one finds that the effective low-energy Hamiltonian corresponding to this Anderson model is of the isotropic form

$$
\hat{H}_{G-G, \mathrm{eff}}=\sum_{\alpha} \sum_{\mathbf{k}, \sigma} \epsilon_{\alpha \mathbf{k}} \psi_{\alpha \mathbf{k} \sigma}^{\dagger} \psi_{\mathbf{k} \sigma}+\sum_{\alpha} J^{\alpha \alpha} \mathbf{s}_{\alpha \alpha} \cdot \boldsymbol{\tau}+J^{s d} \mathbf{s}_{s d} \cdot \boldsymbol{\tau},
$$

schematically shown in Fig. 3.2. Crucially, the cotunnelling terms corresponding to $J^{s R}$ and $J^{d R}$ are absent from the above expression. The reason 
for this is that such terms would involve changing the number of electrons on the right lead, which is prevented by the interactions on the right lead.

As was discussed in the previous section, whenever two leads of a Kondo setup are connected to each other through cotunnelling, one of the channels (the odd channel) decouples from the dot in the limit $T \rightarrow 0$. The two leads should therefore be interpreted together as a single channel. As such, the source and drain leads of the Goldhaber-Gordon setup are understood to be the "left" channel, while the interacting lead provides the "right" channel. The absence of $J^{s R}$ and $J^{d R}$ furthermore ensures that these channels are completely independent of each other, such that the Goldhaber-Gordon setup realizes the isotropic $2 \mathrm{CK}$ model with $J^{L R}=0$. The remaining coupling constants $J^{L L}$ and $J^{R R}$ can be tweaked by tuning the hybridization parameters of the underlying Anderson model, which is in practice done by choosing appropriate values for the gate voltages that determine the tunnelling rates between the different leads and the dot. The Goldhaber-Gordon setup is thus able to access two-channel physics, indeed leading to the first experimental observation of the 2CK state in 2007 [33].

\subsection{The charge two-channel Kondo model}

In addition to the groundbreaking setup from the previous section, there has more recently been a second realization of the $2 \mathrm{CK}$ model by means of a charge two-channel Kondo (C2CK) device [11, 35]. It is this particular setup that forms the primary focus of this work. The C2CK device, as proposed in Refs. [36, 37] and experimentally realized in Ref. [11], fundamentally differs from the Goldhaber-Gordon setup in that does not originate from an Anderson impurity model. In fact, the device does not involve the actual spin of the impurity, and instead makes clever use of a charge-related degree of freedom to construct the 2CK Hamiltonian from the ground up.

Before discussing the corresponding effective model, we describe the components of the $\mathrm{C} 2 \mathrm{CK}$ device as shown in Fig. 3.3. The setup consists of a large metallic island (acting as a quantum dot with a continuous spectrum) connected to two separate metallic leads through quantum point contacts with highly tunable transmission coefficients $t_{L, R}[36,37$. There is a strong perpendicular magnetic field which serves two purposes: (i) the leads and the dot are in the quantum Hall regime, providing unidirectional edge channels; (ii) spin degeneracy is broken both in the dot and the leads, producing spin-polarized fermions. For this reason, we will henceforth omit the spin index. The number of electrons on the quantum dot is controlled by a gate 


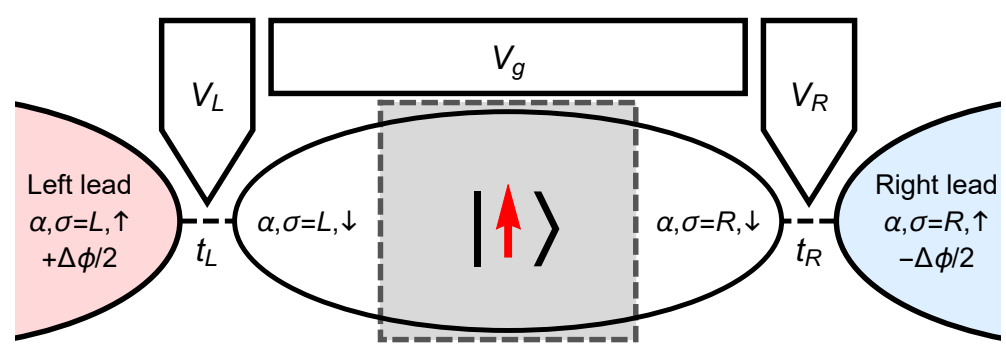

Figure 3.3: Schematic of the C2CK device, where $V_{L, R}$ govern the transmission coefficients $t_{L, R}$, and $V_{g}$ determines the charge on the dot. The dashed grey box denotes a massive "decoherer" that ensures that the left and right ends of the dot are "disconnected" from each other, i.e. there is no coherent transport between these two ends. The otherwise unused spin index of the itinerant states allows for distinction between electrons living on a lead $(\sigma=\uparrow)$ and electrons located on the large island $(\sigma=\downarrow)$, while the island itself has its own pseudospin that indicates the total number of electrons residing on it. The left and right leads can furthermore be kept at a general "potential" $+\Delta \phi / 2$ and $-\Delta \phi / 2$, respectively, where $\Delta \phi$ can for instance be a bias voltage or a temperature gradient.

voltage $V_{g}$. This gate voltage imposes an electrostatic energy $\sim\left(Q+e N^{\prime}\right)^{2}$, where $N^{\prime}$ is a dimensionless parameter proportional to $V_{g}, e$ is the (positive) elementary charge, and $Q$ is the (negative) charge on the quantum dot. If $V_{g}$ is tuned such that $N^{\prime}$ is half-integer, we have a two-fold degeneracy with either $N=N^{\prime}-1 / 2$ or $N+1=N^{\prime}+1 / 2$ electrons on the dot. Given that the charging energy $E_{C}$ is sufficiently large (i.e. $\left.E_{C} \gg k_{B} T\right) 1^{1}$ the dot states are restricted to $|N\rangle,|N+1\rangle$. The last step towards achieving a two-channel situation is to "disconnect" the two ends of the dot and thereby the two leads. This is achieved by adding a massive "decoherer", a metallic region, on top of the dot, which serves as a scatterer for electrons and inhibits coherent transport from one side of the dot to the other.

In order to formulate an effective model for the $\mathrm{C} 2 \mathrm{CK}$ device, we translate all components to the spin language that was also used for the general anisotropic $2 \mathrm{CK}$ model. First, we identify the dot states $|N\rangle,|N+1\rangle$ as pseudospin states $|\downarrow\rangle,|\uparrow\rangle$. Additionally, we label the itinerant electrons residing on the leads as "spin up" and those on the dot as "spin down", see Fig. 3.3. Note that in doing so, we distinguish between itinerant states on

\footnotetext{
${ }^{1}$ The charging energy is the energy cost of having $N-1$ or $N+2$ rather than $N$ or $N+1$ electrons on the dot. This is equal to $E_{C}=\left((3 e / 2)^{2}-(e / 2)^{2}\right) / 2 C=e^{2} / C$, where $C$ is the capacitance of the dot.
} 
Chapter 3. The two-channel Kondo model

Table 3.1: Summary of the mapping between the anisotropic spin 2CK model (with $J_{\lambda}^{L R}=0$ ) and the C2CK device, further elaborated on in the main text. Note especially that the Ising type interactions $J_{z}^{\alpha \alpha}$ do not exist in the C2CK device.

\begin{tabular}{|l||l|l|}
\hline & Spin & Charge \\
\hline Dot states & $|\uparrow\rangle,|\downarrow\rangle$ & $|N+1\rangle,|N\rangle$ \\
Itinerant states & $\psi_{\alpha \sigma}$ & $\psi_{\alpha \uparrow}$ (leads), $\psi_{\alpha \downarrow}$ (dot) \\
Spin-flip interactions & $J_{\perp}^{\alpha \alpha}$ & $t_{\alpha}$ \\
Ising type interactions & $J_{z}^{\alpha \alpha}$ & - \\
Magnetic field & $B$ & $\Delta V_{g}$ \\
\hline
\end{tabular}

the left and on the right side of the dot, which is made possible by virtue of the decoherer. In this pseudospin language, transport through the quantum dot goes via a lead electron tunnelling onto the quantum dot (changing the dot state from $|\downarrow\rangle$ to $|\uparrow\rangle$ while also flipping the "spin" of the itinerant electron), then another leaving the dot again (returning the dot state to $|\downarrow\rangle$ ). Any other transport processes are prohibited due to the presence of the gate voltage and the decoherer. Transport through the quantum dot is therefore equivalent to a sequence of spin-flip processes. From this observation it follows that the local part of the model is given by the first two terms of Eq. (3.4), where the coupling constants $J_{\perp}^{\alpha \alpha}$ depend on the transmission coefficients $t_{\alpha}$, and $J_{\lambda}^{L R}=0$. The dot spin operators $\tau^{ \pm}$are included to enforce the constraints on the number of particles on the dot, and as such can be thought of as a projector. Furthermore, we note that it is also possible to emulate a dot magnetic field by introducing a small detuning $\Delta V_{g}$ in the gate voltage, which adds a preference to one of the dot states over the other. We thus conclude that the full Hamiltonian of the C2CK model is indeed given by Eq. (3.5), but with certain constraints imposed on $J_{\lambda}^{\alpha \beta}$, most importantly $\left.J_{z}^{\alpha \beta}=0\right]^{2}$ The $\mathrm{C} 2 \mathrm{CK}$ device is thus equivalent to a special point of the general anisotropic $2 \mathrm{CK}$ model, the connection between the two models being summarized in Table 3.1. This equivalence forms the basis for the transport calculations of the following chapters.

As a final remark, we note that the redefinition of the spin label for the itinerant states requires careful consideration when applying a bias between

\footnotetext{
${ }^{2}$ In mapping the C2CK device to Eq. 3.5), we have assumed that both pseudospin states (i.e. the lead and dot electrons) have the same density of states and therefore the same Fermi velocity $v_{F}$. As was verified experimentally in Ref. 11, the low-temperature behaviour is not influenced by any asymmetries in $v_{F}$, such that the aforementioned assumption does not lead to loss of generality when studying two-channel physics.
} 
the leads. In particular, in the case of the usual spin $2 \mathrm{CK}$ model, both the spin up and the spin down states live on the leads; for the C2CK device, only the spin up states live on the leads, while the spin down states are by definition located on the dot. As a result, any bias between the leads of a C2CK device acts only on the (pseudo)spin up states, rather than on both spin species. In order to exploit the equivalence between the anisotropic $2 \mathrm{CK}$ model and the $\mathrm{C} 2 \mathrm{CK}$ device, any bias that is applied to the $\mathrm{C} 2 \mathrm{CK}$ device should therefore be incorporated in the $2 \mathrm{CK}$ model as a spin-dependent bias that does not affect spin down.

\subsection{Two-channel physics}

Having covered the anisotropic $2 \mathrm{CK}$ model and its experimental realizations, we will now return to its most general form, Eq. (3.5), and discuss the associated emergent physics. As was already alluded to in Sec. 3.1, the addition of a second channel introduces behaviour that is not present in the ordinary single channel Kondo model, including the possibility of a NFL ground state. The goal of this section is to establish a more complete picture of this two-channel behaviour.

As a starting point, we consider the special point with independent baths $\left(J_{\lambda}^{L R}=0\right)$, symmetric couplings $\left(J_{\lambda}^{L L}=J_{\lambda}^{R R}\right)$ and no local magnetic field $(B=0)$. At this point, both leads attempt to form a Kondo singlet at low temperature, however, the symmetry of the system prevents the leads from succeeding. The system is frustrated, and the residual dot entropy (i.e. the entropy of the full system minus the entropy of the same system without the dot) approaches $S_{\mathrm{imp}} / k_{B}=\frac{1}{2} \ln 2$ as the temperature goes to zero, characteristic of a Majorana degree of freedom [38. This stands in contrast to the vanishing residual entropy of a more general asymmetric setup, when one lead is coupled more strongly to the dot and succeeds in screening the dot by itself. In addition to having a non-zero residual entropy, this special point in parameter space also exhibits NFL behaviour, most notably in the temperature dependence of thermodynamic quantities. In particular, the heat capacity and the magnetic susceptibility are respectively proportional to $T \ln T$ and $\ln T$, contrary to the linear and constant temperature scaling in the case of a Fermi liquid [28, 29, 38]. For this reason, this point is referred to as the NFL quantum critical point.

More generally, if the baths are independent (i.e. $J_{\lambda}^{L R}=0$ ), the model supports several regimes [35, 39], which are illustrated in Fig. 3.4. When the temperature $T$ is large, the coupling between the dot and the leads is 


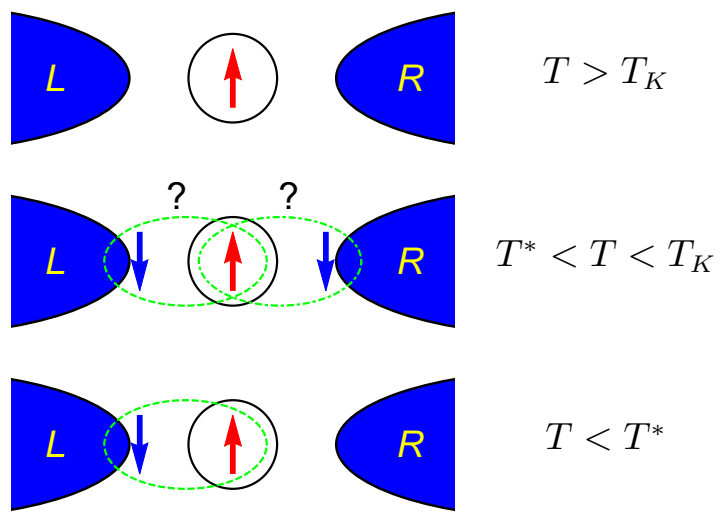

Figure 3.4: Distinct temperature regimes of the 2CK model. Top: local moment fixed point, with weak coupling and relatively little transport. Middle: NFL critical point, characterized by frustration. Bottom: FL regime, corresponding to a transmission node. This particular example originates from a small asymmetry between the leads, such that one lead forms a Kondo singlet with the dot, thereby decoupling the other lead.

weak. As a result, the dot region is now an independent local moment, and transport through the dot is weak as well. The system is thus in the local moment fixed point that we also encountered in the context of the single channel Kondo model, see Sec. 2.3. When the temperature is decreased below $T_{K}$, the Kondo effect sets in and the coupling between the dot and the leads becomes strong. In this region, the leads try to screen the impurity spin. If the couplings are symmetric, the spin is overscreened, resulting in frustration in the sense that both leads compete to screen the spin on equal footing. The system then approaches the NFL critical point as $T \ll T_{K}$, and both leads remain strongly coupled to the dot, enhancing transport between the leads $3^{3}$ However, if there is a small detuning present (e.g. a magnetic field, or an asymmetry in the couplings), there is an additional energy scale $k_{B} T^{*}$ below which the frustration is relieved. As $T \ll T^{*}$, the system will therefore instead flow towards the single channel FL ground state. This FL

\footnotetext{
${ }^{3}$ At first glance, this may seem to go against the common view that the Kondo effect increases the resistivity. It is therefore important to note that we are now referring to transport between two leads, as opposed to transport within a lead. The latter is indeed suppressed by the Kondo effect. However, transport between the leads always involves the dot, such that the Kondo-enhanced coupling of the dot with both leads actually further enables it, thus enhancing the conductance instead of suppressing it.
} 


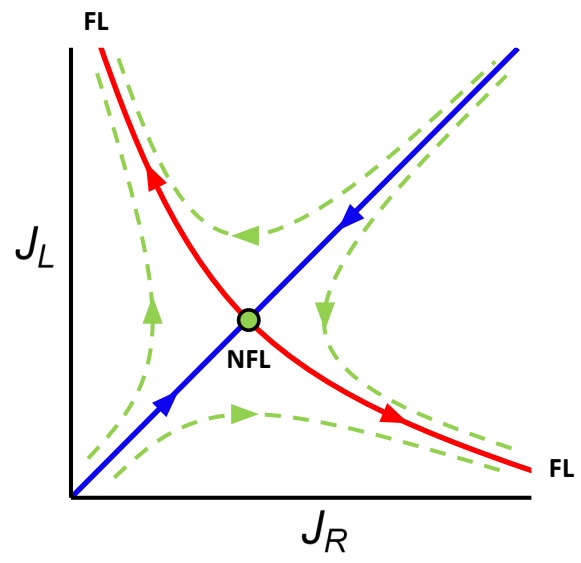

Figure 3.5: Cartoon of the RG flow of the isotropic 2CK model, in absence of cotunnelling between the leads. The solid inward-pointing arrows (blue) correspond to the symmetric case, i.e. the flow towards the NFL fixed point; the solid outwardpointing arrows (red) describe the flow away from this point, towards the FL region. The latter is known as the FL crossover.

ground state does not support any transport through the dot, for example due to a magnetic field locking the dot region into a single spin state, or due to an asymmetry allowing a single lead to screen the dot, decoupling the other lead. The crossover from the NFL region (where the temperature is still sufficiently large for the detuning to be negligible) to the FL region is called the Fermi liquid crossover. Note, however, that this FL crossover only shows universal behaviour for a clear separation of scales, i.e. $T^{*} \ll T_{K}$.

Constraining the model to be isotropic (setting $J_{\lambda}^{\alpha \alpha} \equiv J_{\alpha}$ and $J_{\lambda}^{L R}=0$ for all $\lambda$ ), the above is summarized by the renormalization group (RG) flow illustrated in Fig. 3.5 [27, 40]. Indeed, if the system is exactly symmetric, it stays symmetric upon scaling (blue arrows). However, if there is a small asymmetry, then the system first flows towards the NFL fixed point, but the detuning will keep growing (dashed arrows). This eventually sends the system towards one of the FL regions, meaning that the asymmetry $J_{\Delta} \equiv J_{L}-J_{R}$ (or any other detuning, e.g. the magnetic field) is a relevant parameter. The smaller the asymmetry, the smaller $T^{*}$, so the closer the system flows along the solid arrows. In the limit $T^{*} \ll T \ll T_{K}$, the system starts out infinitesimally close to the NFL fixed point and follows the universal FL crossover line (red arrows) exactly. 
With the isotropic RG flow in mind, it should be noted that the flow diagram of the anisotropic $2 \mathrm{CK}$ model contains additional axes corresponding to anisotropies. Anticipating the exactly solvable point of the model (covered in the next section), let us consider a specific anisotropy, with $J_{x}^{\alpha \beta}=J_{y}^{\alpha \beta} \equiv J_{\perp}^{\alpha \beta}$ and $J_{z}^{L R}=J_{z}^{R L}=0$. With this choice, Eq. 3.2 can be written as

$$
\hat{H}_{\mathrm{loc}}=\sum_{\alpha, \beta} \frac{J_{\perp}^{\alpha \beta}}{2}\left(s_{\alpha \beta}^{+} \tau^{-}+s_{\alpha \beta}^{-} \tau^{+}\right)+\left(J_{z}^{L L} s_{L L}^{z}+J_{z}^{R R} s_{R R}^{z}\right) \tau^{z},
$$

where $s_{\alpha \beta}^{ \pm}=s_{\alpha \beta}^{x} \pm i s_{\alpha \beta}^{y}$ and $\tau^{ \pm}=\tau^{x} \pm i \tau^{y}$ are again the raising and lowering operators corresponding to their respective spins. Returning to our discussion of the RG flow (again setting $J_{\perp}^{L R}=0$ ), the flow diagram of this anisotropic model has two additional axes, representing the anisotropies $\Delta J_{z}^{\alpha \alpha} \equiv J_{z}^{\alpha \alpha}-J_{\perp}^{\alpha \alpha}$. However, contrary to detunings, the anisotropies $\Delta J_{z}^{\alpha \alpha}$ are actually irrelevant parameters [41]. As a result, the system will always end up flowing towards a fixed point in the isotropic plane upon scaling. This in turn means that Ising type interactions $J_{z}^{\alpha \alpha}$ are generated by the RG flow as the energy scale of the system $T / T_{K}$ goes to zero. In terms of Fig. 3.5, systems with a non-zero $\Delta J_{z}^{\alpha \alpha}$ start at a different point out of the plane, but they all end up flowing along the universal FL crossover line. Motivated by this, we will next consider the exactly solvable point of the model (corresponding to a very specific value of $J_{z}^{L L}=J_{z}^{R R} \equiv J_{z}$ ), before using RG arguments to explain how the exact solution can be applied to study the C2CK model (which corresponds to $J_{z}=0$ ).

Finally, we elaborate on the previous statement that the FL crossover is universal [29, 42]. A result of this universality is that the nature of the detuning leading to the FL crossover temperature $T^{*}$ does not influence the FL crossover behaviour: the only difference between detunings is the expression for $T^{*}$ itself, while the behaviour as a function of $T / T^{*}$ remains invariant. This allows us to choose the most convenient detuning in our calculations. In particular, we use the dot magnetic field $B$ as our detuning, keeping the system itself symmetric (i.e. $J_{\perp}^{L L}=J_{\perp}^{R R}$ ).

\subsection{Exactly solvable point of the model}

Making use of the fact that Eq. 3.5) is a one-dimensional model, and restricting the coupling constant $J_{\lambda}^{\alpha \beta}$ to the values used in Eq. (3.11) together with $J_{z}^{L L}=J_{z}^{R R} \equiv J_{z}$, it is possible to map the 2CK model to an equivalent noninteracting resonant level model. This was first done by Emery and Kivelson 
in 1992 [43] and generalized to a non-equilibrium situation (with a timedependent bias between the leads) by Schiller and Hershfield [13. In short, the mapping highlighted in Refs. [13, 43] is done through a series of steps, starting with the bosonization of the fermionic fields, $\psi_{\alpha \sigma}(x) \propto e^{-i \Phi_{\alpha \sigma}(x)}$. Then, a change of basis is performed by taking new linear combinations of the old bosonic fields $\Phi_{L \uparrow}(x), \Phi_{L \downarrow}(x), \Phi_{R \uparrow}(x)$ and $\Phi_{R \downarrow}(x)$; the new fields are referred to as the charge, spin, flavour and spin-flavour modes, defined as

$$
\begin{aligned}
\Phi_{c}(x) & \equiv \frac{1}{2}\left(\Phi_{L \uparrow}(x)+\Phi_{L \downarrow}(x)+\Phi_{R \uparrow}(x)+\Phi_{R \downarrow}(x)\right), \\
\Phi_{s}(x) & \equiv \frac{1}{2}\left(\Phi_{L \uparrow}(x)-\Phi_{L \downarrow}(x)+\Phi_{R \uparrow}(x)-\Phi_{R \downarrow}(x)\right), \\
\Phi_{f}(x) & \equiv \frac{1}{2}\left(\Phi_{L \uparrow}(x)+\Phi_{L \downarrow}(x)-\Phi_{R \uparrow}(x)-\Phi_{R \downarrow}(x)\right), \\
\Phi_{s f}(x) & \equiv \frac{1}{2}\left(\Phi_{L \uparrow}(x)-\Phi_{L \downarrow}(x)-\Phi_{R \uparrow}(x)+\Phi_{R \downarrow}(x)\right) .
\end{aligned}
$$

After rewriting the Hamiltonian in terms of these new bosonic fields, a unitary transformation is performed and the fields are refermionized to find

$$
\begin{aligned}
\hat{H}= & i \hbar v_{F} \sum_{\nu} \int_{-\infty}^{\infty} \mathrm{d} x \psi_{\nu}^{\dagger}(x) \partial_{x} \psi_{\nu}(x)+\frac{J_{\perp}^{L R}}{2 \sqrt{2 \pi a_{0}}}\left(\psi_{f}^{\dagger}(0)-\psi_{f}(0)\right)\left(d^{\dagger}+d\right) \\
& +\frac{J^{+}}{2 \sqrt{2 \pi a_{0}}}\left(\psi_{s f}^{\dagger}(0)+\psi_{s f}(0)\right)\left(d^{\dagger}-d\right) \\
& +\frac{J^{-}}{2 \sqrt{2 \pi a_{0}}}\left(\psi_{s f}^{\dagger}(0)-\psi_{s f}(0)\right)\left(d^{\dagger}+d\right) \\
& +\left(B-\left(J_{z}-2 \pi \hbar v_{F}\right): \psi_{s}^{\dagger}(0) \psi_{s}(0):\right)\left(d^{\dagger} d-\frac{1}{2}\right) .
\end{aligned}
$$

In the above expression, $\nu=c, s, f, s f$, the constant $a_{0}$ is an ultraviolet cut-off originating from the lattice spacing encountered in the bosonization procedure, $d \equiv i \tau^{+}$is a fermionic operator representing the impurity spin, and the coupling constants $J^{ \pm}$are defined as

$$
J^{ \pm} \equiv \frac{1}{2}\left(J_{\perp}^{L L} \pm J_{\perp}^{R R}\right) .
$$

Eq. (3.16) has two important features. First, it immediately follows that the model is quadratic in fermionic operators and therefore non-interacting at the point

$$
J_{z}=2 \pi \hbar v_{F}
$$


where the last term of Eq. (3.16), an interaction term, vanishes. This exactly solvable point is a variation of the so-called Toulouse point of the one-channel Kondo model [44, and we will refer to this particular two-channel Toulouse point as the Emery-Kivelson (EK) point. At the EK point, the model is free and equivalent to a resonant level model. Second, the leads are coupled to Majorana fermions on the dot,

$$
a \equiv \frac{1}{\sqrt{2}}\left(d^{\dagger}+d\right), \quad b \equiv \frac{1}{i \sqrt{2}}\left(d^{\dagger}-d\right),
$$

such that the model is actually equivalent to a Majorana resonant level model when considering the EK point.

Before moving on, a remark on the stability of the EK point is in order. In an RG sense, this exactly solvable point (i.e. with the values of $J_{\lambda}^{\alpha \beta}$ as chosen in Eq. (3.11), together with $J_{z}=2 \pi \hbar v_{F}$ ) can only be stable under one of two conditions [13]: either

$$
J^{-}=J_{\perp}^{L R}=0,
$$

or

$$
J^{+}=0 .
$$

These conditions corresponds to diagonals of the flow diagram, similar to the one from Fig. 3.5. Indeed, deviations away from these diagonals are relevant parameters, destroying the condition $J_{z}^{L L}=J_{z}^{R R}$ upon scaling. As we are interested in the case when there is no cotunnelling between the leads, together with the remaining coupling constants having the same sign (i.e. both being antiferromagnetic), we will focus on the condition from Eq. (3.20). Note that the a Majorana fermion now completely decouples from the Hamiltonian if $B=0$. This indicates that the NFL critical point indeed has an uncoupled Majorana degree of freedom on the dot, accounting for the non-zero residual entropy of $\ln \sqrt{2}$.

Considering the stable EK point with $J_{\perp}^{L R}=0$ and symmetric couplings $J_{\perp}^{L L}=J_{\perp}^{R R} \equiv J_{\perp}$, we now have

$$
\hat{H}=\sum_{\nu} \sum_{k} \epsilon_{k} \psi_{\nu, k}^{\dagger} \psi_{\nu, k}+g_{\perp}\left(\psi_{s f}^{\dagger}(0)+\psi_{s f}(0)\right)\left(d^{\dagger}-d\right)+\frac{B}{2}\left(d^{\dagger} d-d d^{\dagger}\right),
$$

where $\epsilon_{k}=\hbar v_{F} k$ and $g_{\perp} \equiv J_{\perp} / 2 \sqrt{2 \pi a_{0}}$. As we shall see in the next section, this Hamiltonian can also be used in the context of a C2CK device, thus providing a way to perform exact calculations on the experimental setup. 


\subsection{Applicability of the Emery-Kivelson point}

As we have seen in the preceding sections, the exactly solvable EK point and the effective $\mathrm{C} 2 \mathrm{CK}$ model are both special points of the anisotropic 2CK model. Setting $J_{\lambda}^{L R}=0$ as usual, the only difference between these two special points is in their values for $J_{z}$. The distinction between the two is nevertheless important: the anisotropic $2 \mathrm{CK}$ model is only non-interacting for a very specific finite value of $J_{z}$, while the concept of Ising type interactions (i.e. any non-zero $J_{z}$ ) does not exist in the C2CK model. However, the observation that the EK point and the $\mathrm{C} 2 \mathrm{CK}$ model are so similar implies that the exact solvability of the former can be used as a starting point to analyse the latter. Indeed, as we have seen in Sec. 3.4, both the experimental setup (where $J_{z}=0$ ) and a setup prepared at the EK point (with $J_{z}=2 \pi \hbar v_{F}$ ) flow towards the same fixed point as $T / T_{K}$ goes to zero. Most importantly, for non-zero $T^{*}$ they both end up flowing along the same universal FL crossover line upon scaling. Any results from the region $T \ll T_{K}$ that are obtained at the EK point are therefore also applicable to the $\mathrm{C} 2 \mathrm{CK}$ experiments. Accordingly, the strategy for all subsequent calculations concerning transport in the $\mathrm{C} 2 \mathrm{CK}$ device is to first solve the problem at the EK point (which can be done exactly), then consider the limit $T / T_{K} \rightarrow 0$ and note the corresponding results are also valid for the C2CK device. Note, however, that the same arguments hold for any energy scale, so we have to be careful that all energy scales are taken to be much smaller than the Kondo energy scale $k_{B} T_{K}$, see Fig. 3.6. The simplest way to incorporate this is taking the limit $T_{K} \rightarrow \infty$ in all of the EK point results. The disadvantage of having to take this limit is that we cannot use the EK point to fully investigate the transition from the local moment fixed point to the NFL critical point, which happens at $T \sim T_{K}$. On the other hand, the $T_{K} \rightarrow \infty$ limit

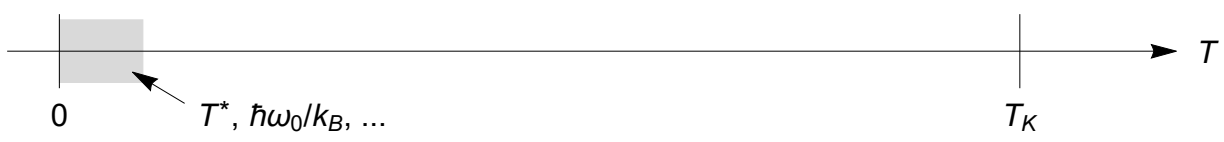

Figure 3.6: Rough sketch of the temperature scales. All energy scales (such as those associated with the FL crossover temperature $T^{*}$ and the external driving frequency $\omega_{0}$ ) are chosen to be much smaller than the Kondo energy scale $k_{B} T_{K}$, such that all temperature scales other than $T_{K}$ are located in the grey region. We restrict our study to this region (i.e. $T / T_{K} \rightarrow 0$ ), where the results from the EK point become exact for the $\mathrm{C} 2 \mathrm{CK}$ setup. 
does permit us to keep the FL crossover temperature $T^{*}$ finite, so we can still investigate the FL crossover in an exact manner.

Despite the fact that this method does not give the correct $\mathrm{C} 2 \mathrm{CK}$ results for $T \gtrsim T_{K}$, it is nevertheless possible to use the EK point as a starting point to study the true behaviour in this region. In order to do so, one has to expand the EK point solution about $J_{z}=2 \pi \hbar v_{F}$ [45]. In our case, such perturbations are a function of $T / T_{K}$, and will only vanish if $T / T_{K} \rightarrow 0$. Taking all of the above into account and restricting ourselves to the FL crossover, we use the Hamiltonian from Eq. (3.22) as the starting point for all of the $\mathrm{C} 2 \mathrm{CK}$ transport calculations that are to follow. 


\section{Chapter 4}

\section{Transport in two-channel setups}

Given their geometry, one of the most natural phenomena to study in $2 \mathrm{CK}$ setups is the transport between the different leads of these setups. Additional motivation is provided by the fact that the $2 \mathrm{CK}$ model supports a NFL fixed point with a decoupled Majorana degree of freedom, suggesting possible emergence of unconventional NFL and Majorana signatures in the observable currents. In this chapter we will cover the methods for calculating the transport properties of both the Goldhaber-Gordon and the $\mathrm{C} 2 \mathrm{CK}$ setups, paying special attention to transport of charge and heat. For the Goldhaber-Gordon setup the calculations are immediately carried out, while the complexity of those corresponding to the $\mathrm{C} 2 \mathrm{CK}$ device demands a more elaborate analysis. The latter will be addressed in the following chapters.

\subsection{Transport in the Goldhaber-Gordon setup}

First, we return to the Goldhaber-Gordon setup that was introduced in Sec. 3.2. Since the source and drain leads are the only proper reservoirs in this setup (the third lead being finite), we will specifically consider currents flowing between these two leads. Recalling that the source and drain leads together form a single channel in the associated effective 2CK model, we will thus be looking at transport within a single channel, as opposed to transport between two separate channels.

For models consisting of two non-interacting leads that are connected to an interacting central region by means of simple hybridization terms, the electronic transport between the leads is described by the Meir-Wingreen formula [46]. The derivation of this formula is based on the Keldysh formalism for non-equilibrium systems [47]; in Sec. 4.2.4 we will elaborate on the underlying methods by deriving a similar formula for the special case of the $\mathrm{C} 2 \mathrm{CK}$ device. As the model of Goldhaber-Gordon's 2CK setup from Eq. (3.9) does indeed have the required structure, we will study its transport properties by applying the Meir-Wingreen formula. Our first step is to 
impose a purely local impurity (i.e. $V_{\alpha \mathbf{k}}=V_{\alpha}$, where $\alpha=s, d$ ) and a constant density of states on the leads (i.e. $\rho_{\alpha}(\epsilon)=\rho_{\alpha}$, following from the fact that the leads can be understood as effectively one-dimensional). With these constraints, the level broadening functions $\Gamma_{\alpha}(\epsilon) \equiv \pi \rho_{\alpha}(\epsilon)\left|V_{\alpha}(\epsilon)\right|^{2}$ become completely independent of the energy $\epsilon$, thereby guaranteeing that the system has proportionate coupling, $\Gamma_{s}(\epsilon) \propto \Gamma_{d}(\epsilon)$. In the case of proportionate coupling, the Meir-Wingreen formula assumes the simplified form [46, 48]

$$
I_{Q}=\frac{2}{h} \int_{-\infty}^{\infty} \mathrm{d} \epsilon\left[n_{F}^{s}(\epsilon)-n_{F}^{d}(\epsilon)\right] Q(\epsilon) t(\epsilon),
$$

where $I_{Q}$ is the current corresponding to the "charge" of interest $Q(\epsilon)$ (e.g. electric charge or energy) that is transported from the source to the drain lead by the electrons hopping through the central region. In the above expression, $\epsilon$ is the energy measured with respect the equilibrium chemical potential of the full system, $n_{F}^{\alpha}(\epsilon)$ is the Fermi-Dirac distribution,

$$
n_{F}(\epsilon)=\frac{1}{e^{\epsilon / k_{B} T}+1},
$$

associated with lead $\alpha$ (assumed to be in thermal equilibrium), and

$$
t(\epsilon)=2 \pi \frac{\Gamma_{s} \Gamma_{d}}{\Gamma_{s}+\Gamma_{d}} A(\epsilon)
$$

plays the role of the T-matrix, with $A(\epsilon)$ being the spectral function of the impurity site (summed over both spin species). Note that the third lead (i.e. the large metallic island) does not appear directly in the above expressions despite the fact that it is part of the "central region". Nevertheless, this interacting lead can strongly influence the impurity site, thus entering the equation through $A(\epsilon)$. Intuitively, Eq. (4.1) can be understood by recognizing that the integrand is roughly the product of the density of the surplus of electrons on the source lead, the charge carried by each electron, and the transfer rate of the electrons, therefore integrating to the total transported charge per unit time.

Starting from Eq. 4.1), we will now calculate the electrical current (corresponding to $Q(\epsilon)=-e$ ) due to a small bias voltage $V$ between the leads and the energy current (with $Q(\epsilon)=\epsilon$ ) resulting from a small temperature gradient $\Delta T$, both in the limit $T \rightarrow 0$. The former can be incorporated by applying a symmetrized voltage $\pm V / 2$ to the source and drain leads (such 
that $\left.n_{F}^{s / d}(\epsilon)=n_{F}(\epsilon \pm e V / 2)\right)$, while in the latter case we write $T \rightarrow T \pm \Delta T / 2$ for the respective leads. In the limit of small $T, V$ and $\Delta T$, the product $\left[n_{F}^{s}(\epsilon)-n_{F}^{d}(\epsilon)\right] Q(\epsilon)$ is strongly peaked around $\epsilon=0$, such that the currents are determined by the low-energy behaviour of $t(\epsilon)$. First considering the FL regime, the appropriate expansion of the T-matrix is given by [42]

$$
t(\epsilon)=t(0)+\frac{1}{2} t^{\prime \prime}(0) \epsilon^{2}+\mathcal{O}\left(\epsilon^{4}\right) .
$$

With the help of the identities

$$
\begin{aligned}
\int_{-\infty}^{\infty} \mathrm{d} \epsilon n_{F}(\epsilon)\left(1-n_{F}(\epsilon)\right) & =k_{B} T, \\
\int_{-\infty}^{\infty} \mathrm{d} \epsilon \epsilon^{2} n_{F}(\epsilon)\left(1-n_{F}(\epsilon)\right) & =\frac{\pi^{2} k_{B}^{3} T^{3}}{3}, \\
\int_{-\infty}^{\infty} \mathrm{d} \epsilon \epsilon^{4} n_{F}(\epsilon)\left(1-n_{F}(\epsilon)\right) & =\frac{7 \pi^{4} k_{B}^{5} T^{5}}{15},
\end{aligned}
$$

we find

$$
\begin{aligned}
I_{c} & =-\frac{2 e}{h} \int_{-\infty}^{\infty} \mathrm{d} \epsilon\left[n_{F}(\epsilon+e V / 2)-n_{F}(\epsilon-e V / 2)\right] t(\epsilon) \\
& =\frac{2 e^{2} V}{h k_{B} T} \int_{-\infty}^{\infty} \mathrm{d} \epsilon n_{F}(\epsilon)\left(1-n_{F}(\epsilon)\right)\left(t(0)+\frac{1}{2} t^{\prime \prime}(0) \epsilon^{2}+\mathcal{O}\left(\epsilon^{4}\right)\right)+\mathcal{O}\left(V^{2}\right) \\
& =\frac{2 e^{2}}{h}\left(t(0)+\frac{\pi^{2} k_{B}^{2} T^{2}}{6} t^{\prime \prime}(0)+\mathcal{O}\left(T^{4}\right)\right) V+\mathcal{O}\left(V^{2}\right)
\end{aligned}
$$

for the electrical current due to a small bias voltage $V$ between the leads in the FL regime. Analogously, the energy current driven by a temperature gradient $\Delta T$ is given by

$$
\begin{aligned}
I_{E} & =\frac{2 \Delta T}{h k_{B} T^{2}} \int_{-\infty}^{\infty} \mathrm{d} \epsilon \epsilon^{2} n_{F}(\epsilon)\left(1-n_{F}(\epsilon)\right)\left(t(0)+\frac{1}{2} t^{\prime \prime}(0) \epsilon^{2}+\mathcal{O}\left(\epsilon^{4}\right)\right)+\mathcal{O}\left(\Delta T^{2}\right) \\
& =\frac{2 \pi^{2} k_{B}^{2} T}{3 h}\left(t(0)+\frac{7 \pi^{2} k_{B}^{2} T^{2}}{10} t^{\prime \prime}(0)+\mathcal{O}\left(T^{4}\right)\right) \Delta T+\mathcal{O}\left(\Delta T^{2}\right)
\end{aligned}
$$


If $t(0)$ is non-zero, the low-temperature currents can be obtained from the above expressions by only keeping the zeroth order term in the temperature expansions, resulting in expressions that also hold beyond the FL regime.

As was discussed in Sec. 3.4, the 2CK model has two possible ground states, namely the NFL fixed point and the one-channel FL ground state. In the case of the Goldhaber-Gordon setup, the FL ground state furthermore supports two microscopic realizations: the effective channel consisting of the source and drain leads is either the only channel that remains coupled to the impurity (thus screening the impurity on its own), or it is decoupled. Since all transport goes through the dot, the latter does not support any transport between the source and drain leads. This corresponds to $t(0)=0$ in Eqs. 4.8) and (4.9), and the low-temperature behaviour of the currents of a setup with a decoupled ground state is therefore determined by $t^{\prime \prime}(0)$ instead of $t(0)$. On the other hand, the strongly coupled one-channel ground state and the NFL fixed point respectively correspond to $t(0)=\gamma$ and $t(0)=$ $\gamma / 2$ [29, 42, 48, 49], where $\gamma=4 \Gamma_{s} \Gamma_{d} /\left(\Gamma_{s}+\Gamma_{d}\right)^{2}$ is a geometrical factor that depends on the details of the setup. As such, the qualitative temperature dependences of the currents are determined by whether the geometry allows the source/drain channel to remain coupled to the dot as $T \rightarrow 0$.

Finally, we touch on the possibility of thermopower in the GoldhaberGordon setup, i.e. an electrical current that is driven by a temperature gradient. In this case, the product $\left[n_{F}^{s}(\epsilon)-n_{F}^{d}(\epsilon)\right] Q(\epsilon)$ is odd in $\epsilon$, such that the even part of $t(\epsilon)$ vanishes upon integration. Since the linear term is absent from the T-matrix expansion from Eq. (4.4), we can conclude that the thermopower goes to zero with the temperature for either of the FL ground states. Moreover, following the discussion from Sec. 3.6, the critical theory of the NFL fixed point is given by the EK point Hamiltonian from Eq. (3.22) with $B=0$ and the particle-hole symmetric dispersion $\epsilon_{k}=\hbar v_{F} k$. The overall particle-hole symmetry of this critical theory guarantees that $t(\epsilon)$ is even in $\epsilon$ at the NFL fixed point, such that the thermopower does not only vanish for the two FL ground states, but at the NFL fixed point as well.

\subsection{Transport in the charge two-channel Kondo setup: methods and key quantities}

Contrary to the Goldhaber-Gordon setup, the Meir-Wingreen formula cannot be used to study transport in the C2CK device. The reason for this is that the C2CK model does not originate from an Anderson impurity model (or anything similar) and therefore lacks the structure from which the Meir- 
Wingreen formula was originally derived. The purpose of this section is to introduce two alternative methods for calculating the transport properties of the $\mathrm{C} 2 \mathrm{CK}$ device, respectively based on Keldysh formalism and linear response theory. Both methods rely on the exactly solvable point of the 2CK model in order to obtain exact results, thus using Eq. (3.22) as the "bare" model Hamiltonian. In the following, we will first discuss the general concept of conserved charges that are coupled to a bias by a simple potential term and introduce the associated current operators, before performing the Emery-Kivelson mapping to obtain the effective current operators in the equivalent non-interacting theory. Afterwards, we will cover the propagators of the model and how they can be exploited to calculate the time-dependent electrical currents using Keldysh techniques. Finally, the special case of a temperature gradient will be discussed, which cannot enter the Hamiltonian directly and instead requires linear response theory.

\subsubsection{Potentials and current operators}

The most important ingredients for transport calculations are the current operators. Before we define the current operators, however, let us first see how a general potential gradient between the two leads enters the Hamiltonian, using the electric charge and a bias voltage as an example. In order to avoid unnecessary clutter and to keep the dot region out of the potential term in the Hamiltonian, we apply the bias voltage symmetrically: the left lead is acted upon by a bias voltage of $V / 2$, while the right lead is subject to a bias voltage of $-V / 2$, such that the total voltage drop between the leads is equal to $V$. Given that $\sigma=\uparrow$ refers to the electrons in the leads, the additional term in the Hamiltonian due to a bias voltage is given by

$$
\hat{H}_{V}=-\frac{e V}{2} \int_{-\infty}^{\infty} \mathrm{d} x\left[\psi_{L \uparrow}^{\dagger}(x) \psi_{L \uparrow}(x)-\psi_{R \uparrow}^{\dagger}(x) \psi_{R \uparrow}(x)\right],
$$

which can simply be written as $\hat{H}_{V}=\hat{q} V$, with

$$
\hat{q} \equiv \frac{1}{2}\left(\hat{q}_{L}-\hat{q}_{R}\right), \quad \hat{q}_{\alpha}=-e \int_{-\infty}^{\infty} \mathrm{d} x \psi_{\alpha \uparrow}^{\dagger}(x) \psi_{\alpha \uparrow}(x)=-e \hat{N}_{\alpha} .
$$

Here, the object $\hat{q}_{\alpha}$ is the operator of the total charge on lead $\alpha=L, R$, while $\hat{N}_{\alpha}$ is the number operator corresponding to this lead. The above contribution can be generalized to a general "charge" operator $\hat{Q}_{\alpha}$ on lead 
$\alpha$ that is being coupled to a general "potential" drop between the leads $\Delta \phi$. Also allowing the charge and the potential to be time-dependent, we obtain the following general minimal coupling contribution to the Hamiltonian:

$$
\hat{H}_{\phi}(t)=\hat{Q}(t) \Delta \phi(t), \quad \hat{Q}(t) \equiv \frac{1}{2}\left(\hat{Q}_{L}(t)-\hat{Q}_{R}(t)\right) .
$$

Additionally, we define the current operator $\hat{I}_{Q}$ corresponding to the general charge $\hat{Q}$. Applying the continuity equation and imposing that the total charge is conserved, the operator corresponding to the current leaving lead $\alpha$ is given by $\hat{I}_{Q_{\alpha}}=-\frac{\mathrm{d} \hat{Q}_{\alpha}}{\mathrm{d} t}$. Now the most sensible way to define the current flowing through the dot region is to take the average of the current leaving the left lead and the current entering the right lead. This gives

$$
\hat{I}_{Q} \equiv \frac{1}{2}\left(-\frac{\mathrm{d} \hat{Q}_{L}}{\mathrm{~d} t}+\frac{\mathrm{d} \hat{Q}_{R}}{\mathrm{~d} t}\right)=-\frac{\mathrm{d} \hat{Q}}{\mathrm{~d} t}=\frac{i}{\hbar}[\hat{Q}, \hat{H}],
$$

with $\hat{H}$ being the full Hamiltonian (i.e. Eq. 3.22 with the addition of $\hat{H}_{\phi}$ ). For charge transport, we thus have $\hat{I}_{c}=\frac{e}{2} \frac{\mathrm{d}}{\mathrm{d} t}\left(\hat{N}_{L}-\hat{N}_{R}\right)$, while for energy transport the current operator is given by $\left.\hat{I}_{E}=-\frac{1}{2} \mathrm{~d} d \hat{H}_{L}-\hat{H}_{R}\right)$, where $\hat{H}_{\alpha}$ is the part of the Hamiltonian corresponding to lead $\alpha$. From the first law of thermodynamics at constant volume, $\mathrm{d} E=\mathrm{d} Q+\mu \mathrm{d} N$ ( $\mathrm{d} Q$ referring to heat and $\mu$ being the chemical potential), it follows that the heat current operator is given by $\hat{I}_{h}=\hat{I}_{E}-\mu \hat{I}_{N}$.

\subsubsection{Emery-Kivelson mapping of the current operators}

As was discussed in Sec. 3.6, our main strategy concerning the C2CK device is to use the exactly solvable EK point. It is therefore necessary to apply the Emery-Kivelson mapping [43] (as briefly outlined in Sec. 3.5) to the current operators as well. We will do so by performing the mapping on the "charge" operators $\hat{Q}_{c}=-\frac{e}{2}\left(\hat{N}_{L}-\hat{N}_{R}\right)$ and $\hat{Q}_{E}=\frac{1}{2}\left(\hat{H}_{L}-\hat{H}_{R}\right)$, then working out the commutators of these operators with the full (mapped) Hamiltonian from Eq. (3.22) 1. More details on the bosonization and refermionization [30, 50, 51] used in the mapping procedure can be found in Appendix A.1.

The first part of the mapping procedure is the introduction of a bosonic field $\Phi_{\alpha \sigma}(x)$ for each of the fermionic fields $\psi_{\alpha \sigma}(x)$ :

$$
\psi_{\alpha \sigma}(x)=\frac{1}{\sqrt{2 \pi a_{0}}} e^{i \phi_{\alpha \sigma}} e^{-i \Phi_{\alpha \sigma}(x)} ;
$$

\footnotetext{
${ }^{1}$ It is also possible to calculate the commutators first and only then going through the mapping procedure, but that is a much more difficult route.
} 
the exponentials $e^{i \phi_{\alpha \sigma}}$ act as Klein factors to enforce the correct anticommutation relations between the fermionic fields. Following the usual bosonization prescription, the various components of the charge operators transform according to

$$
\begin{aligned}
\int_{-\infty}^{\infty} \mathrm{d} x \psi_{\alpha \sigma}^{\dagger}(x) \psi_{\alpha \sigma}(x) & =\frac{1}{2 \pi} \int_{-\infty}^{\infty} \mathrm{d} x \partial_{x} \Phi_{\alpha \sigma}(x), \\
\int_{-\infty}^{\infty} \mathrm{d} x \psi_{\alpha \sigma}^{\dagger}(x) \partial_{x} \psi_{\alpha \sigma}(x) & =-\frac{i}{4 \pi} \int_{-\infty}^{\infty} \mathrm{d} x\left(\partial_{x} \Phi_{\alpha \sigma}(x)\right)^{2},
\end{aligned}
$$

where normal ordering of the fermionic fields is implied. Plugging the above into the charge operators and writing the resulting expressions in terms of the $\nu=c, s, f, s f$ fields from Eqs. (3.12)-(3.15), we find

$$
\begin{aligned}
& \hat{Q}_{c}=-\frac{e}{4 \pi} \int_{-\infty}^{\infty} \mathrm{d} x\left(\partial_{x} \Phi_{f}(x)+\partial_{x} \Phi_{s f}(x)\right) \\
& \hat{Q}_{E}=\frac{\hbar v_{F}}{8 \pi} \int_{-\infty}^{\infty} \mathrm{d} x\left(\partial_{x} \Phi_{c}(x)+\partial_{x} \Phi_{s}(x)\right)\left(\partial_{x} \Phi_{f}(x)+\partial_{x} \Phi_{s f}(x)\right) .
\end{aligned}
$$

The next step of the Emery-Kivelson mapping procedure is the unitary transformation $\hat{\mathcal{O}} \rightarrow \hat{U} \hat{\mathcal{O}} \hat{U}^{\dagger}$, with $\hat{U}=e^{i \chi_{s} \tau^{z}}$ and $\chi_{s} \equiv \Phi_{s}(0)-\phi_{s}$. Using the commutation relation

$$
\left[\Phi_{\mu}(x), \partial_{x} \Phi_{\nu}\left(x^{\prime}\right)\right]=2 \pi i \delta_{\mu, \nu} \delta\left(x-x^{\prime}\right)
$$

together with $d \equiv i \tau^{+}$(such that $\tau^{z}=-\left(d^{\dagger} d-1 / 2\right)$ ), it is straightforward to show that

$$
\begin{aligned}
\hat{Q}_{E} \rightarrow & \frac{\hbar v_{F}}{8 \pi} \int_{-\infty}^{\infty} \mathrm{d} x\left(\partial_{x} \Phi_{c}(x)+\partial_{x} \Phi_{s}(x)\right)\left(\partial_{x} \Phi_{f}(x)+\partial_{x} \Phi_{s f}(x)\right) \\
& +\left.\frac{\hbar v_{F}}{4}\left(d^{\dagger} d-\frac{1}{2}\right)\left(\partial_{x} \Phi_{f}(x)+\partial_{x} \Phi_{s f}(x)\right)\right|_{x=0}
\end{aligned}
$$

under this unitary transformation, while $\hat{Q}_{c}$ remains unchanged. The final step of the mapping procedure consists of refermionization. Using relations 
similar to those involved in the initial bosonization step and noting that

$$
\int_{-\infty}^{\infty} \mathrm{d} x \psi_{\mu}^{\dagger}(x) \psi_{\mu}(x) \psi_{\nu}^{\dagger}(x) \psi_{\nu}(x)=\frac{1}{4 \pi^{2}} \int_{-\infty}^{\infty} \mathrm{d} x\left(\partial_{x} \Phi_{\mu}(x)\right)\left(\partial_{x} \Phi_{\nu}(x)\right)
$$

for $\mu \neq \nu$ (normal ordering of the fermionic fields again implied, see the appendix), we find that the charge operators finally become

$$
\begin{aligned}
\hat{Q}_{c}= & -\frac{e}{2} \int_{-\infty}^{\infty} \mathrm{d} x\left(\psi_{f}^{\dagger}(x) \psi_{f}(x)+\psi_{s f}^{\dagger}(x) \psi_{s f}(x)\right) \\
\hat{Q}_{E}= & \frac{\pi \hbar v_{F}}{2} \int_{-\infty}^{\infty} \mathrm{d} x\left(\psi_{c}^{\dagger}(x) \psi_{c}(x)+\psi_{s}^{\dagger}(x) \psi_{s}(x)\right)\left(\psi_{f}^{\dagger}(x) \psi_{f}(x)+\psi_{s f}^{\dagger}(x) \psi_{s f}(x)\right) \\
& +\frac{\pi \hbar v_{F}}{2}\left(: \psi_{f}^{\dagger}(0) \psi_{f}(0):+: \psi_{s f}^{\dagger}(0) \psi_{s f}(0):\right)\left(d^{\dagger} d-\frac{1}{2}\right)
\end{aligned}
$$

We now calculate the current operators by Fourier transforming the charge operators to momentum space and evaluating the commutators with the Hamiltonian from Eq. (3.22). Starting with electric transport:

$$
\begin{aligned}
\hat{I}_{c} & =-\frac{i e}{2 \hbar} \sum_{k}\left[\psi_{f, k}^{\dagger} \psi_{f, k}+\psi_{s f, k}^{\dagger} \psi_{s f, k}, \hat{H}\right] \\
& =-\frac{i e g_{\perp}}{2 \hbar \sqrt{L}} \sum_{k}\left(\psi_{s f, k}^{\dagger}-\psi_{s f, k}\right)\left(d^{\dagger}-d\right),
\end{aligned}
$$

where $L$ is a length scale originating from Fourier transforming the $\psi_{\nu}$ fields. ${ }^{2}$ Although more cumbersome, the energy current operator can be obtained in the same way, leading to

$$
\begin{aligned}
\hat{I}_{E}= & \frac{i \pi v_{F} g_{\perp}}{2 L^{3 / 2}} \sum_{k, k^{\prime}, k^{\prime \prime}}\left(\psi_{c, k^{\prime}}^{\dagger} \psi_{c, k^{\prime \prime}}+\psi_{s, k^{\prime}}^{\dagger} \psi_{s, k^{\prime \prime}}\right)\left(\psi_{s f, k}^{\dagger}-\psi_{s f, k}\right)\left(d^{\dagger}-d\right) \\
& +\frac{i \pi v_{F} g_{\perp}}{4 L^{3 / 2}} \sum_{k, k^{\prime}, k^{\prime \prime}}\left(2 \psi_{f, k^{\prime}}^{\dagger} \psi_{f, k^{\prime \prime}}+\delta_{k^{\prime}, k^{\prime \prime}}\right)\left(\psi_{s f, k}^{\dagger}+\psi_{s f, k}\right)\left(d^{\dagger}+d\right) \\
& +\frac{i \pi v_{F}}{4 L} \sum_{k, k^{\prime}}\left(\epsilon_{k^{\prime}}-\epsilon_{k}\right)\left(\psi_{f, k}^{\dagger} \psi_{f, k^{\prime}}+\psi_{s f, k}^{\dagger} \psi_{s f, k^{\prime}}\right)\left(d^{\dagger} d-d d^{\dagger}\right) .
\end{aligned}
$$

\footnotetext{
${ }^{2}$ By writing the sums over $x$ as integrals, we implicitly rescaled the position space fields by a factor $1 / \sqrt{a}$, where $a$ is the lattice constant of the underlying lattice. In terms of the Fourier transform convention from Eq. 2.3), this requires replacing $N$ (the number of lattice sites on a given lead) with $L=a N$ (the length of the one-dimensional chain).
} 
The most striking difference between the two current operators is that the energy current operator (and by extension the heat current operator) is much more complicated than the charge current operator. This originates from the fact that heat transport itself is a more complicated concept: while electric transport only involves charge-carrying excitations, heat transport involves all modes that are supported by the system. As a result, the mapping of a strongly interacting system to an effective non-interacting model comes at the price of a significantly more complicated heat current operator. In terms of the Emery-Kivelson mapping procedure, this fundamental difference between charge and heat transport emerges during the unitary transformation. In particular, the operator corresponding to electric charge does not pick up additional terms due to the fact that the spin modes $\Phi_{s}$ do not carry charge and therefore commute with $\hat{Q}_{c}$. On the other hand, the spin modes do carry energy, resulting in $\hat{Q}_{E}$ obtaining several additional terms upon performing the unitary transformation. Indeed, the entirety of the second and third lines of Eq. 4.25) can be traced back to this step.

\subsubsection{Equilibrium propagators of the model}

In the case that a simple general charge $Q$ is minimally coupled to some bias in accordance with Eq. 4.12), the observable time- and temperaturedependent current can be calculated by taking the expectation value of the associated current operator with respect to the full Hamiltonian. This requires finding the propagators of the model. Following the usual functional integral formalism to construct the action corresponding to Eq. (3.22) and integrating out the charge and spin modes, we obtain the following expression for the full equilibrium Green function of the system $3^{3}$

$$
\mathbf{G} \equiv\left(\begin{array}{ccc}
\mathbf{L} & \mathbf{G}_{l d} & \mathbf{G}_{l r} \\
\mathbf{G}_{d l} & \mathbf{D} & \mathbf{G}_{d r} \\
\mathbf{G}_{r l} & \mathbf{G}_{r d} & \mathbf{R}
\end{array}\right)=\left(\begin{array}{ccc}
\mathbf{L}_{0}^{-1} & -\mathbf{g}_{\perp} / \hbar & 0 \\
-\mathbf{g}_{\perp}^{\dagger} / \hbar & \mathbf{D}_{0}^{-1} & 0 \\
0 & 0 & \mathbf{R}_{0}^{-1}
\end{array}\right)^{-1}
$$

independent of the basis of the components. Here, $\mathbf{L}, \mathbf{R}$, and $\mathbf{D}$ are the full Green functions corresponding to the spin-flavour modes, the flavour modes,

${ }^{3}$ For notational convenience, we will from now on use the similarities with a regular resonant level model to identify "spin-flavour" as "left", and "flavour" as "right", leading to the uses of the letters $L$ and $R$ in the expressions. Including the latter in Eq. 4.26) is not necessary in absence of a channel asymmetry (as the flavour modes are then decoupled from the rest of the system), but we include it here for completeness. Also note that this left/right nomenclature is unrelated to the original left and right leads that we started with. 
and the dot, respectively. Moreover, $\mathbf{L}_{0}, \mathbf{R}_{0}$ and $\mathbf{D}_{0}$ are the corresponding "bare" Green functions in absence of tunnelling $\left.\right|^{4}$ while $\mathbf{g}_{\perp}$ governs the coupling between the spin-flavour modes and the dot. Block inversion of the right-hand side of Eq. (4.26) gives

$$
\begin{aligned}
\mathbf{D} & =\left(\mathbf{D}_{0}^{-1}-\mathbf{\Sigma}_{d}\right)^{-1}, \quad \mathbf{\Sigma}_{d} \equiv \frac{1}{\hbar^{2}} \mathbf{g}_{\perp}^{\dagger} \cdot \mathbf{L}_{0} \cdot \mathbf{g}_{\perp}, \\
\mathbf{G}_{l d} & =\frac{1}{\hbar} \mathbf{L}_{0} \cdot \mathbf{g}_{\perp} \cdot \mathbf{D}, \\
\mathbf{L} & =\mathbf{L}_{0}+\frac{1}{\hbar^{2}} \mathbf{L}_{0} \cdot \mathbf{g}_{\perp} \cdot \mathbf{D} \cdot \mathbf{g}_{\perp}^{\dagger} \cdot \mathbf{L}_{0},
\end{aligned}
$$

where $\boldsymbol{\Sigma}_{d}$ can be identified as the self-energy of the dot. All possible propagators can thus be calculated from the full Green function on the dot, together with bare quantities. This essentially reduced the problem of finding the currents to obtaining a single Green function.

In order to calculate the necessary Green functions, it is important to keep in mind that all tunnelling happens via Majorana modes, i.e. operators such as $a \equiv\left(d^{\dagger}+d\right) / \sqrt{2}$ and $b \equiv\left(d^{\dagger}-d\right) / i \sqrt{2}$. This Majorana character can properly be incorporated by switching to the Nambu spinor basis, for example working with $\mathbf{d}^{\dagger} \equiv\left(d^{\dagger} d\right)$. Doing so, the action becomes

$$
S=\frac{\hbar}{2} \int_{-\infty}^{\infty} \mathrm{d} t \bar{\psi} \cdot \mathbf{G}^{-1} \cdot \psi,
$$

where $\psi, \bar{\psi}$ are vectors containing all of the Grassmann fields, and the factor $1 / 2$ is included to compensate for the fact that switching to the Nambu basis effectively doubles each term. In momentum space, all components of the tunnelling matrix (labelled by index $k$ ) can be deduced from Eq. 3.22, and are given by

$$
\mathbf{g}_{\perp, k}=\frac{g_{\perp}}{\sqrt{L}}\left(\begin{array}{ll}
-1 & 1 \\
-1 & 1
\end{array}\right) \equiv \frac{g_{\perp}}{\sqrt{L}} \mathbf{g},
$$

independent of $k$. Moreover, all of the momentum space components of the Green functions are $2 \times 2$ matrices as well. Using the observation that the bare Hamiltonian (i.e. in absence of tunnelling) is symmetric in $\nu=f, s f$, together with the fact that it does not contain any superconducting pairs

\footnotetext{
${ }^{4}$ The word "bare" can have two different meanings: sometimes it refers to the system in absence of a bias, $\Delta \phi=0$; at other times it refers to the absence of tunnelling, $g_{\perp}=0$. We elaborate on the meaning whenever the meaning is not clear from the context.
} 
such as $\psi_{\nu, k} \psi_{\nu, k^{\prime}}$, the components of the bare propagators are found to be of the form

$$
\mathbf{L}_{0, k k^{\prime}}=\mathbf{R}_{0, k k^{\prime}}=\delta_{k, k^{\prime}} \mathbf{L}_{0, k}=\delta_{k, k^{\prime}}\left(\begin{array}{cc}
L_{0, k, 1} & 0 \\
0 & L_{0, k, 2}
\end{array}\right), \quad \mathbf{D}_{0}=\left(\begin{array}{cc}
D_{0,1} & 0 \\
0 & D_{0,2}
\end{array}\right) .
$$

In momentum space, the Green functions from Eqs. (4.28) and 4.29) become

$$
\begin{aligned}
\mathbf{G}_{l d, k} & =\frac{g_{\perp}}{\hbar \sqrt{L}} \mathbf{L}_{0, k} \cdot \mathbf{g} \cdot \mathbf{D}, \\
\mathbf{L}_{k k^{\prime}} & =\delta_{k, k^{\prime}} \mathbf{L}_{0, k}+\frac{g_{\perp}^{2}}{\hbar^{2} L} \mathbf{L}_{0, k} \cdot \mathbf{g} \cdot \mathbf{D} \cdot \mathbf{g}^{\dagger} \cdot \mathbf{L}_{0, k^{\prime}},
\end{aligned}
$$

with the dot self-energy being equal to

$$
\boldsymbol{\Sigma}_{d}=\frac{g_{\perp}^{2}}{\hbar^{2}} \mathbf{g}^{\dagger} \cdot\left(\frac{1}{L} \sum_{k} \mathbf{L}_{0, k}\right) \cdot \mathbf{g} \equiv \frac{g_{\perp}^{2}}{\hbar^{2}} \mathbf{g}^{\dagger} \cdot \mathbf{L}_{0}^{\prime} \cdot \mathbf{g} .
$$

For future reference, we also introduce the Majorana Green functions on the dot, corresponding to the Majorana fermions $a$ and $b$; they are given by

$$
\begin{aligned}
D_{a a} & =\frac{1}{2}\left(D_{11}+D_{12}+D_{21}+D_{22}\right), \\
D_{b b} & =\frac{1}{2}\left(D_{11}-D_{12}-D_{21}+D_{22}\right), \\
D_{a b} & =\frac{1}{2 i}\left(D_{11}-D_{12}+D_{21}-D_{22}\right), \\
D_{b a} & =\frac{1}{2 i}\left(-D_{11}-D_{12}+D_{21}+D_{22}\right),
\end{aligned}
$$

where $D_{i j}$ are the original components of the $2 \times 2$ matrix D. Finally, it should be noted that all of the above fields and Green functions have implied time-dependence.

Whenever the system is driven out of equilibrium, evaluating the propagators requires the use of Keldysh techniques, see the next section. Moreover, the presence of a minimal coupling term could potentially change the structure of the Hamiltonian, thereby invalidating most of this section's equations. However, when the system is in equilibrium (which is for example the case in linear response theory), the above propagators can be evaluated using Matsubara techniques. In absence of a bias and in terms of fermionic Matsubara frequencies $\omega_{n}$, the necessary Green functions are given by

$$
\mathbf{L}_{0, k}\left(i \omega_{n}\right)=\hbar\left(\begin{array}{cc}
\left(i \hbar \omega_{n}-\epsilon_{k}\right)^{-1} & 0 \\
0 & \left(i \hbar \omega_{n}+\epsilon_{k}\right)^{-1}
\end{array}\right),
$$




$$
\mathbf{D}\left(i \omega_{n}\right) \equiv \mathbf{G}_{d d}\left(i \omega_{n}\right)=\int_{-\infty}^{\infty} \mathrm{d} \epsilon \frac{\boldsymbol{\rho}(\epsilon)}{i \hbar \omega_{n}-\epsilon}, \quad \boldsymbol{\rho}(\epsilon) \equiv-\frac{1}{\pi} \operatorname{Im}\left[\mathbf{D}^{\mathrm{R}}(\epsilon)\right],
$$

where $\boldsymbol{\rho}$ can be interpreted as a density of states [4, and the retarded dot Green function is given by

$$
\mathbf{D}^{\mathrm{R}}(\epsilon)=\frac{\hbar}{\epsilon(\epsilon+i \Gamma)-B^{2}}\left(\begin{array}{cc}
\epsilon+B+\frac{i}{2} \Gamma & \frac{i}{2} \Gamma \\
\frac{i}{2} \Gamma & \epsilon-\frac{i}{B} \Gamma+\frac{i}{2} \Gamma
\end{array}\right) .
$$

Here, the parameter $\Gamma$ has been introduced for notational convenience and for later reference; it is defined as

$$
\Gamma \equiv 2 g_{\perp}^{2} \frac{\mathrm{d} k}{\mathrm{~d} \epsilon_{k}}=\frac{2 g_{\perp}^{2}}{\hbar v_{F}}=\frac{J_{\perp}^{2}}{4 \pi a_{0} \hbar v_{F}},
$$

and is in essence identical to the level broadening functions that were encountered in Sec. 4.1. A full derivation of the dot propagator from Eq. 4.42 can be found in Appendix A.2.

\subsubsection{Non-equilibrium techniques: Keldysh formalism}

With the help of the mapped current operators and the propagator structure from the previous sections, we will now introduce our first method for studying transport in the $\mathrm{C} 2 \mathrm{CK}$ device, particularly concentrating on charge transport. For this first method, we will utilize non-equilibrium techniques to calculate the observable currents of the $2 \mathrm{CK}$ model at the EK point in presence of a finite bias voltage. The procedure is inspired by the elaborate investigation of time-dependent transport through a central region by Jauho, Wingreen and Meir in Ref. [52, and is essentially an extension of their methods to transport involving Majorana degrees of freedom instead of fermionic ones. This extension to the EK point was first performed by Schiller and Hershfield in the context of the spin variant of the 2CK model in Refs. [12, 13, which we will now adapt to the C2CK device by applying the appropriate bias term and current operator.

In the case of charge transport, the full Hamiltonian (including the minimal coupling term with a time-dependent bias voltage) is given by

$$
\begin{aligned}
\hat{H}= & \sum_{\nu=f, s f} \sum_{k}\left(\epsilon_{k}-\frac{e V(t)}{2}\right) \psi_{\nu, k}^{\dagger} \psi_{\nu, k}+\frac{g_{\perp}}{\sqrt{L}} \sum_{k}\left(\psi_{s f, k}^{\dagger}+\psi_{s f, k}\right)\left(d^{\dagger}-d\right) \\
& +\frac{B}{2}\left(d^{\dagger} d-d d^{\dagger}\right) .
\end{aligned}
$$


Note that the $\nu=c, s$ modes have been omitted (integrated out) because they do not couple to the potential or the impurity, nor do they appear in the current operator from Eq. (4.24), implying that they do not influence any of the transport quantities of interest. The most important observation for solving the model is that the above Hamiltonian is still quadratic, such that it can be treated exactly for any $V(t)$. Moreover, the minimally coupled Hamiltonian from Eq. (4.44) has exactly the same form as the bare one from Eq. (3.22), with the bias voltage only acting as a time-dependent shift in the dispersion relation. The non-equilibrium propagators (subject to a bias voltage) therefore have the same structure as the equilibrium propagators from the previous section. However, as the Hamiltonian contains an explicit time-dependence, standard Matsubara techniques cannot be used, and we will instead use Keldysh techniques [47] to calculate the necessary correlators. More information about the Keldysh structure employed in this section can be found in Appendix A.3.

Following the Keldysh prescription, each Green function gains an additional matrix structure of the form

$$
\mathbf{G}=\left(\begin{array}{cc}
\mathbf{G}^{\mathrm{R}} & \mathbf{G}^{\mathrm{K}} \\
0 & \mathbf{G}^{\mathrm{A}}
\end{array}\right)
$$

where $\mathbf{G}^{\mathrm{R} / \mathrm{A}}$ are the retarded and advanced Green functions, while $\mathbf{G}^{\mathrm{K}}$ is the so-called Keldysh component of the Green function. The desired two-point functions are proportional to the Keldysh Green functions and can in general be obtained in the following way:

$$
\left\langle\psi_{\mu} \psi_{\nu}^{\dagger}\right\rangle=\frac{i}{2} G_{\mu \nu}^{\mathrm{K}}
$$

Returning to the current operator from Eq. (4.24), we can thus write the expectation value of the charge current as follows:

$$
I_{c}(t) \equiv\left\langle\hat{I}_{c}\right\rangle(t)=-\frac{e g_{\perp}}{4 \hbar \sqrt{L}} \sum_{k}\left(G_{l d, k, 11}^{\mathrm{K}}+G_{l d, k, 22}^{\mathrm{K}}-G_{l d, k, 12}^{\mathrm{K}}-G_{l d, k, 21}^{\mathrm{K}}\right)(t, t) .
$$

Here, the first two indices (i.e. $l d$ ) are the ones from Eq. (4.26), the final two indices refer to the Nambu spinor structure, and the letter $\mathrm{K}$ refers the Keldysh structure. From Eq. (4.33), it furthermore follows that the required Green function is of the form 5

$$
\frac{1}{\sqrt{L}} \sum_{k} \mathbf{G}_{l d, k}(t, t)=\frac{g_{\perp}}{\hbar}\left(\mathbf{L}_{0}^{\prime} \cdot \mathbf{g} \cdot \mathbf{D}\right)(t, t),
$$

\footnotetext{
${ }^{5}$ Matrix multiplication of the form $(\mathbf{A} \cdot \mathbf{B})\left(t, t^{\prime}\right)$ is short for $\int_{-\infty}^{\infty} \mathrm{d} t^{\prime \prime} \mathbf{A}\left(t, t^{\prime \prime}\right) \cdot \mathbf{B}\left(t^{\prime \prime}, t^{\prime}\right)$.
} 
where the dot self-energy is given by Eq. 4.35). In terms of the Majorana propagators from Eqs. 4.36-4.39), this sum becomes

$$
\begin{aligned}
\frac{1}{\sqrt{L}} \sum_{k} \mathbf{G}_{l d, k} & =\frac{g_{\perp}}{\hbar}\left(\begin{array}{ll}
L_{0,1}^{\prime}\left(D_{21}-D_{11}\right) & L_{0,1}^{\prime}\left(D_{22}-D_{12}\right) \\
L_{0,2}^{\prime}\left(D_{21}-D_{11}\right) & L_{0,2}^{\prime}\left(D_{22}-D_{12}\right)
\end{array}\right) \\
& =\frac{g_{\perp}}{\hbar}\left(\begin{array}{ll}
L_{0,1}^{\prime}\left(-D_{b b}+i D_{b a}\right) & L_{0,1}^{\prime}\left(D_{b b}+i D_{b a}\right) \\
L_{0,2}^{\prime}\left(-D_{b b}+i D_{b a}\right) & L_{0,2}^{\prime}\left(D_{b b}+i D_{b a}\right)
\end{array}\right) .
\end{aligned}
$$

Plugging the above back into Eq. (4.47), we find the following expression for the charge current:

$$
I_{c}(t)=\frac{e g_{\perp}^{2}}{2 \hbar^{2}}\left(\left(L_{0,1}^{\prime}-L_{0,2}^{\prime}\right) D_{b b}\right)^{\mathrm{K}}(t, t),
$$

where $L_{0,1}^{\prime}$ and $L_{0,2}^{\prime}$ can respectively be interpreted as particle and hole propagators. It should be noted that the system becomes completely timeindependent in the dc limit (corresponding to $V(t)=$ const.), such that Green functions assume the form $G\left(t, t^{\prime}\right)=G\left(t-t^{\prime}\right)$. As a result, the dc current loses its time-dependence as expected.

The difficulty in finding propagators in any non-equilibrium problem is related to finding the corresponding non-equilibrium density matrix. In thermal equilibrium, the density matrix is given by $\hat{\rho}_{0}=\exp \left[-(\hat{H}-\mu \hat{N}) / k_{B} T\right]$, while out of equilibrium one has to solve the quantum Boltzmann equation. The latter is usually not possible in an exact manner. We will circumvent this problem by assuming that the bare flavour and spin-flavour modes are in thermal equilibrium, with the bias voltage only acting on the tunnel junctions between the leads and the dot. Any corrections to this assumption are inversely proportional to the system size, such that they go to zero in the thermodynamic limit. Using what we have seen in Sec. 4.2.3, the only full Green function that we need for the calculation of the currents is the one on the dot. While this coupled dot is still very much out of equilibrium, we can make use of Eqs. (4.27) and (4.35) to see that the non-equilibrium behaviour can be expressed in terms of bare Green functions, thereby avoiding any direct calculation of the non-equilibrium density matrix.

With the above in mind, evaluation of Eq. 4.50 nevertheless requires a method for dealing with the Keldysh structure. From Eq. 4.45), it immediately follows that the components necessary for calculating the currents are of the form

$$
(\mathbf{A} \cdot \mathbf{B})^{\mathrm{K}}=\mathbf{A}^{\mathrm{R}} \cdot \mathbf{B}^{\mathrm{K}}+\mathbf{A}^{\mathrm{K}} \cdot \mathbf{B}^{\mathrm{A}} .
$$

In order to evaluate such expressions, we can employ the usual methods for the retarded and advanced Green functions, while the Keldysh components 
have to be calculated using the auxiliary relation

$$
\mathbf{G}^{\mathrm{K}}=\mathbf{G}^{\mathrm{R}} \cdot \mathbf{F}-\mathbf{F} \cdot \mathbf{G}^{\mathrm{A}} .
$$

The Hermitian matrix $\mathbf{F}$ can in principle be found by solving the quantum Boltzmann equation. In thermal equilibrium, the fluctuation-dissipation theorem (FDT) holds, and the solution is given by

$$
\mathbf{F}(\epsilon)=\left(1-2 n_{F}(\epsilon)\right) \mathbb{1} \equiv f(\epsilon) \mathbb{1},
$$

see e.g. Ref. 47] for a formal derivation. It is important to stress that this expression for the matrix $\mathbf{F}$ is only valid in thermal equilibrium and cannot be used in general. However, as discussed above, the flavour and spin-flavour modes both act as baths in the thermodynamic limit, such that the bare Green functions corresponding to these modes can be assumed to satisfy the FDT. For these modes, the bias voltage can therefore simply be interpreted as a time-dependent shift in the chemical potential [52].

We close this section with a couple of remarks. (i) The expression of the current from Eq. (4.50) is very similar to the proportionate coupling MeirWingreen formula that was discussed in Sec. 4.1. The only differences are that Eq. 4.50 includes the possibility of a time-dependent potential, involves Majorana degrees of freedom, and originates from charge and current operators that are specific to the $\mathrm{C} 2 \mathrm{CK}$ device. (ii) The methods from this section are not restricted to electric transport, and can in principle be applied to any minimally coupled charge and any current. Note, however, that the operator of a different minimally coupled charge could potentially introduce an interaction term to the Hamiltonian through the Emery-Kivelson mapping, thereby dismissing the exact solvability and necessitating the use of perturbation theory.

\subsubsection{Linear response theory}

In the previous sections, we have discussed currents resulting from a bias directly entering the Hamiltonian. However, the case of heat transport introduces additional problems, so the goal of this section is to formulate a method that is well-suited to study heat transport that is caused by a temperature gradient between the leads. The reason that we cannot directly calculate the expectation value of the heat current operator is two-fold. First, the temperature gradient cannot enter the Hamiltonian in the same way as the bias voltage, since temperature is a concept that has no microscopic meaning. The usual solution for this problem is to instead apply different 
temperatures to the Fermi-Dirac distributions of each lead. This brings us to the second problem: as was discussed in Sec. 4.2.4, direct calculation of any current depends heavily on the mapped lead modes being in thermal equilibrium. This means that they must all obey the Fermi-Dirac distribution with a certain temperature. However, these $\nu=c, s, f, s f$ modes are composite modes that consist of modes living on both leads (see Eqs. (3.12)-(3.15)). As a result, if the original leads are in thermal equilibrium but at different temperatures, then the mapped lead modes cannot be assigned a definite temperature and are therefore no longer in thermal equilibrium. Studying heat transport thus requires a way to circumvent these two problems. The solution is found in linear response theory [53], where we only consider the linear susceptibility to a certain bias, evaluated at zero bias.

As a starting point, we again consider a general "charge" $\hat{Q}$ coupled to a general "potential" $\Delta \phi$, previously introduced in Eq. 4.12. For real time $t$, the full expectation value of the current corresponding to $\hat{Q}$ is given by

$$
\left\langle\hat{I}_{Q}\right\rangle(t)=\int_{-\infty}^{\infty} \mathrm{d} t^{\prime} \chi\left(t, t^{\prime}\right) \Delta \phi\left(t^{\prime}\right)+\mathcal{O}\left(\Delta \phi^{2}\right)
$$

Using the fact that the bare system is time-independent (such that the susceptibility obeys $\left.\chi\left(t, t^{\prime}\right)=\chi\left(t-t^{\prime}\right)\right)$, the Fourier transform of this equation is simply

$$
\left\langle\hat{I}_{Q}\right\rangle(\omega)=\chi(\omega) \Delta \phi(\omega)+\mathcal{O}\left(\Delta \phi^{2}\right) .
$$

Furthermore defining $\langle\ldots\rangle_{0}$ to be the expectation value in absence of a potential gradient (i.e. the static equilibrium case), the susceptibility can be obtained from

$$
\chi(\omega)=\frac{i}{\hbar \omega}\left(C^{\mathrm{R}}(\omega)-C^{\mathrm{R}}(0)\right),
$$

where $C^{\mathrm{R}}(\omega)$ is the Fourier transform of the retarded current autocorrelator,

$$
C^{\mathrm{R}}(\omega)=\int_{-\infty}^{\infty} \mathrm{d} \Delta t C^{\mathrm{R}}(\Delta t) e^{i \omega \Delta t}=\int_{-\infty}^{\infty} \mathrm{d} \Delta t\left(-i \theta(\Delta t)\left\langle\left[\hat{I}_{Q}(\Delta t), \hat{I}_{Q}(0)\right]\right\rangle_{0}\right) e^{i \omega \Delta t} .
$$

The above is known as the Kubo formula [54, 55]; a short derivation of this formula can be found in Appendix A.4. It provides a way to calculate the linear susceptibility of some current $I_{Q}$ to a potential drop $\Delta \phi$ between the leads, purely in terms of "bare" equilibrium quantities. In order to evaluate 
the right-hand side of Eq. 4.56), we will first calculate the imaginary time correlation function, defined as

$$
C^{\tau}\left(\tau_{1}-\tau_{2}\right) \equiv-\left\langle T_{\tau} \hat{I}_{Q}\left(\tau_{1}\right) \hat{I}_{Q}\left(\tau_{2}\right)\right\rangle_{0},
$$

where $T_{\tau}$ is the time ordering operator for the imaginary time $\tau=i t$. We do this by switching to bosonic Matsubara frequencies $\Omega_{n} \equiv \frac{2 \pi n}{\hbar \beta}$ (with $\beta \equiv$ $\left.1 / k_{B} T\right)$, using the bosonic frequencies over the fermionic ones because the current operators only contain even powers of fermionic operators:

$$
C^{\tau}\left(i \Omega_{n}\right)=\int_{0}^{\hbar \beta} \mathrm{d} \tau C^{\tau}(\tau) e^{i \Omega_{n} \tau} .
$$

The susceptibility in terms of real frequency $\omega$ is now found by performing analytic continuation on the correlation function, writing $C^{\tau}\left(i \Omega_{n>0}\right) \rightarrow$ $\left.C\left(\omega+i 0^{+}\right) \equiv C^{\mathrm{R}}(\omega)[4]\right]^{6}$ Finally, the dc limit can be obtained by taking the limit $\omega \rightarrow 0$, in particular $\chi_{\mathrm{dc}}=\lim _{\omega \rightarrow 0} \chi(\omega)$.

Since the Kubo formula only involves the evaluation of equilibrium expectation values, utilizing this formalism inherently avoids the problem of the modes not being in equilibrium. However, the other problem (i.e. a temperature gradient not being able to enter the Hamiltonian) requires a different approach. The solution was first proposed by Luttinger in 1964 [56]. The idea behind Luttinger's solution is that the temperature is not the only field that couples to the energy density; in particular, a gravitational field does so as well. The advantage of a gravitational field is that it can enter the Hamiltonian in the general way outlined in Eq. (4.12). In absence of a chemical potential $\mu$, the heat current subject to both a temperature gradient and a gradient in a gravitational field $\psi$ is phenomenologically given by

$$
I_{h}=\chi_{T} \frac{\Delta T}{T}+\chi_{\psi} \Delta \psi
$$

to lowest order in the gradients, where $\Delta T$ and $\Delta \psi$ respectively denote the drop in temperature and gravitational field between the leads. Luttinger showed that the corresponding linear susceptibilities are equal to each other, $\chi_{T}=\chi_{\psi}$. Therefore, one can calculate the susceptibility $\chi_{\psi}$ due to a gravitational field in absence of a temperature gradient, then use this result to find

\footnotetext{
${ }^{6}$ The poles and branch cuts of the the analytically continued function $C(z \in \mathbb{C})$ are all located on the real axis, such that $C(z)$ can be a different analytic function for $\operatorname{Im}[z]>0$ and $\operatorname{Im}[z]<0$. Since we are interested in points with $\operatorname{Im}\left[z=\omega+i 0^{+}\right]>0$, we only have to consider the points on the positive imaginary axis, i.e. $i \Omega_{n>0}$.
} 
the heat current due to a temperature gradient in absence of a gravitational field. To rephrase, we can find the heat current due to a temperature gradient by first calculating $\chi_{\psi}$ (which is in turn done by considering a minimal coupling term), then setting $\chi_{T}=\chi_{\psi}$ and calculating $I_{h}=\chi_{T} \Delta T / T$. While the full heat current is no longer exact (since we neglect the higher order terms in $\Delta T / T$ ), the linear susceptibility $\chi_{T}$ can be obtained exactly.

Finally, we consider the linear response currents in presence of both a bias voltage and a temperature gradient, again with $\mu=0$. Phenomenologically, the equations for the charge and heat currents can be written as

$$
\left(\begin{array}{l}
I_{c} \\
I_{h}
\end{array}\right)=\left(\begin{array}{ll}
\chi_{11} & \chi_{12} \\
\chi_{21} & \chi_{22}
\end{array}\right)\left(\begin{array}{c}
V \\
\Delta T / T
\end{array}\right)
$$

where $\chi_{11} \sim\left\langle\hat{I}_{c} \hat{I}_{c}\right\rangle_{0}$ and $\chi_{22} \sim\left\langle\hat{I}_{E} \hat{I}_{E}\right\rangle_{0}$ are respectively the isolated charge and heat susceptibilities, while $\chi_{12} \sim\left\langle\hat{I}_{c} \hat{I}_{E}\right\rangle_{0}$ and $\chi_{21} \sim\left\langle\hat{I}_{E} \hat{I}_{c}\right\rangle_{0}$ represent thermopower [56]. In this more general situation, the heat current is consequently given by $I_{h}=\chi_{21} V+\chi_{22} \Delta T / T$. Defining the heat conductance $\kappa$ through $I_{h} \equiv \kappa \Delta T$, the heat conductance can assume two different forms: (i) in absence of a bias voltage (i.e. $V=0$ ), the heat conductance satisfies $T \kappa=\chi_{22}$; (ii) in absence of an electrical current (i.e. $I_{c}=0$ ), the heat conductance is given by $T \kappa=\chi_{22}-\chi_{12} \chi_{21} / \chi_{11}$. In the latter case, a bias voltage of $V=-\left(\chi_{12} / \chi_{11}\right) \Delta T / T$ has been applied to cancel the thermopower that emerges as a result of the non-zero temperature gradient. In general, it is therefore necessary to specify which quantity (either $V$ or $I_{c}$ ) is kept equal to zero when evaluating the heat conductance. 


\section{Chapter 5}

\section{Time-dependent charge transport at the exactly solvable point}

Following the general discussion of the methods concerning transport in the C2CK device from the previous chapter, we will now perform an elaborate technical analysis of charge transport due to a time-dependent bias voltage at the exactly solvable EK point, using the Keldysh techniques that were introduced in Sec. 4.2.4. In doing so, we will continue to follow the core methods from Refs. [12, 13, 52, although it should be noted that the application of these methods to the context of the $\mathrm{C} 2 \mathrm{CK}$ device requires a different execution and physical interpretation, thus leading to different results. Three distinct cases will be discussed: a constant bias voltage (corresponding to dc), a sinusoidal driving voltage (representing ac), and a sudden quantum quench that can be used to study relaxation to a new steady state.

\subsection{Electrical current as a voltage-dependent integral}

In order to calculate the charge current for the bias voltages listed above, it is useful to write the current as a voltage-dependent integral. We will do so by starting with the result from Eq. 4.50 and working out the local bare lead propagator $\mathbf{L}_{0}^{\prime}$ in the wide-band limit, i.e. assuming that the dispersion relation $\epsilon_{k}=\hbar v_{F} k$ holds for all momenta $k$ from $-\infty$ to $\infty$. In the wide-band limit, the continuum limit can furthermore be obtained by writing

$$
\frac{1}{L} \sum_{k} \rightarrow \int_{-\infty}^{\infty} \frac{\mathrm{d} k}{2 \pi}=\frac{1}{v_{F}} \int_{-\infty}^{\infty} \frac{\mathrm{d} \epsilon_{k}}{2 \pi \hbar} .
$$

Both of these limits will be assumed for all subsequent calculations. First, for the retarded and advanced components of $\mathbf{L}_{0}^{\prime}$ we use the following relation:

$$
\left(\delta\left(t-t^{\prime}\right)\left(i \partial_{t^{\prime}}-\epsilon_{k}\left(t^{\prime}\right) / \hbar \pm i 0^{+}\right)\right)^{-1}=\mp i \theta\left( \pm\left(t-t^{\prime}\right)\right) e^{-\frac{i}{\hbar} \int_{t^{\prime}}^{t} \mathrm{~d} t^{\prime \prime} \epsilon_{k}\left(t^{\prime \prime}\right)},
$$


which can easily be checked using the definition of the inverse. In absence of a bias voltage, the retarded and advanced components of the local bare propagator of the spin-flavour modes are thus given by

$$
\begin{aligned}
& \left.\left(\mathbf{L}_{0}^{\prime}\right)^{\mathrm{R} / \mathrm{A}}\left(t, t^{\prime}\right)\right|_{V=0} \\
& =\frac{1}{v_{F}} \int_{-\infty}^{\infty} \frac{\mathrm{d} \epsilon_{k}}{2 \pi \hbar}\left(\begin{array}{cc}
\left(\delta\left(t-t^{\prime}\right)\left(i \partial_{t^{\prime}}-\epsilon_{k} / \hbar \pm i 0^{+}\right)\right)^{-1} & 0 \\
0 & \left(\delta\left(t-t^{\prime}\right)\left(i \partial_{t^{\prime}}+\epsilon_{k} / \hbar \pm i 0^{+}\right)\right)^{-1}
\end{array}\right) \\
& =\mp \frac{i}{v_{F}} \theta\left( \pm\left(t-t^{\prime}\right)\right) \int_{-\infty}^{\infty} \frac{\mathrm{d} \epsilon_{k}}{2 \pi \hbar}\left(\begin{array}{cc}
e^{-\frac{i \epsilon_{k}}{\hbar}\left(t-t^{\prime}\right)} & 0 \\
0 & e^{\frac{i \epsilon_{k}}{\hbar}\left(t-t^{\prime}\right)}
\end{array}\right) \\
& =\mp \frac{i}{2 v_{F}} \delta\left(t-t^{\prime}\right) \mathbb{1}_{2},
\end{aligned}
$$

where $\mathbb{1}_{2}$ is the $2 \times 2$ identity matrix in the Nambu spinor basis. Turning on the bias voltage (which is done by writing $\epsilon_{k} \rightarrow \epsilon_{k}-e V(t) / 2$ in the exponent, see Eq. 4.44) ) leaves the right-hand side unmodified:

$$
\begin{aligned}
\left(\mathbf{L}_{0}^{\prime}\right)^{\mathrm{R} / \mathrm{A}}\left(t, t^{\prime}\right) & =\mp \frac{i}{2 v_{F}} \delta\left(t-t^{\prime}\right)\left(\begin{array}{cc}
e^{\frac{i e}{2 \hbar} \int_{t^{\prime}}^{t} \mathrm{~d} t^{\prime \prime} V\left(t^{\prime \prime}\right)} & 0 \\
0 & e^{-\frac{i e}{2 \hbar} \int_{t^{\prime}}^{t} \mathrm{~d} t^{\prime \prime} V\left(t^{\prime \prime}\right)}
\end{array}\right) \\
& =\mp \frac{i}{2 v_{F}} \delta\left(t-t^{\prime}\right) \mathbb{1}_{2} .
\end{aligned}
$$

For the Keldysh component of $\mathbf{L}_{0}^{\prime}$, we turn to Eqs. 4.52 and 4.53):

$$
\begin{array}{rl}
\left(\mathbf{L}_{0}^{\prime}\right)^{\mathrm{K}}\left(t, t^{\prime}\right) & =-\frac{i}{v_{F}} \int_{-\infty}^{\infty} \frac{\mathrm{d} \epsilon}{2 \pi \hbar}\left(f(\epsilon) e^{-\frac{i \epsilon}{\hbar}\left(t-t^{\prime}\right)+\frac{i e}{2 \hbar} \int_{t^{\prime}}^{t} \mathrm{~d} t^{\prime \prime} V\left(t^{\prime \prime}\right)}\right. \\
0 & 0 \\
0 & \left.f(-\epsilon) e^{\frac{i \epsilon}{\hbar}\left(t-t^{\prime}\right)-\frac{i e}{2 \hbar} \int_{t^{\prime}}^{t} \mathrm{~d} t^{\prime \prime} V\left(t^{\prime \prime}\right)}\right) \\
& =-\frac{i}{v_{F}} \int_{-\infty}^{\infty} \frac{\mathrm{d} \epsilon}{2 \pi \hbar} f(\epsilon) e^{-\frac{i \epsilon}{\hbar}\left(t-t^{\prime}\right)}\left(\begin{array}{cc}
e^{\frac{i e}{2 \hbar} \int_{t^{\prime}}^{t} \mathrm{~d} t^{\prime \prime} V\left(t^{\prime \prime}\right)} & 0 \\
0 & \left.e^{-\frac{i e}{2 \hbar} \int_{t^{\prime}}^{t} \mathrm{~d} t^{\prime \prime} V\left(t^{\prime \prime}\right)}\right),
\end{array}\right)
\end{array}
$$

where we dropped the subscript $k$ from the integration variable. Note that the components $\mathbf{D}^{\mathrm{R} / \mathrm{A}}$ of the full dot Green function are completely determined by $\mathbf{D}_{0}^{\mathrm{R} / \mathrm{A}}$ and $\left(\mathbf{L}_{0}^{\prime}\right)^{\mathrm{R} / \mathrm{A}}$, such that the bias voltage can only enter 
through Keldysh Green functions. Consequently, the equilibrium expression for $\mathbf{D}^{\mathrm{R}}(\epsilon)$ from Eq. 4.42 remains valid even in presence of a bias voltage.

Let us now use the above expressions and their properties to further evaluate the charge current. Plugging everything back into Eq. 4.50) and using Eq. (4.51) together with $\left(\mathbf{L}_{0}^{\prime}\right)^{\mathrm{R}} \propto \mathbb{1}_{2}$ to see that the term proportional to $D_{b b}^{\mathrm{K}}$ vanishes, we find

$$
\begin{aligned}
I_{c}(t)= & \frac{e g_{\perp}^{2}}{2 \hbar^{2}} \int_{-\infty}^{\infty} \mathrm{d} t^{\prime}\left(\left(L_{0,1}^{\prime}\right)^{\mathrm{K}}\left(t, t^{\prime}\right)-\left(L_{0,2}^{\prime}\right)^{\mathrm{K}}\left(t, t^{\prime}\right)\right) D_{b b}^{\mathrm{A}}\left(t^{\prime}, t\right) \\
= & -\frac{i e g_{\perp}^{2}}{2 \hbar^{2} v_{F}} \int_{-\infty}^{\infty} \frac{\mathrm{d} \epsilon}{2 \pi \hbar} f(\epsilon) \\
& \times \int_{-\infty}^{\infty} \mathrm{d} t^{\prime} e^{-\frac{i \epsilon}{\hbar}\left(t-t^{\prime}\right)}\left(e^{\frac{i e}{2 \hbar} \int_{t^{\prime}}^{t} \mathrm{~d} t^{\prime \prime} V\left(t^{\prime \prime}\right)}-e^{-\frac{i e}{2 \hbar} \int_{t^{\prime}}^{t} \mathrm{~d} t^{\prime \prime} V\left(t^{\prime \prime}\right)}\right) D_{b b}^{\mathrm{A}}\left(t^{\prime}, t\right) .
\end{aligned}
$$

Inspired by the work on a regular resonant level model from Ref. [52], a different (and often more convenient) way of writing the charge current is obtained by noting that $\hat{I}_{c}$ is a Hermitian operator, together with the observation that $\left(L_{0,2}^{\prime}\right)^{\mathrm{K}}\left(t, t^{\prime}\right)=\left(\left(L_{0,1}^{\prime}\right)^{\mathrm{K}}\left(t, t^{\prime}\right)\right)^{*}$. The latter is a consequence of the fact that $f(\epsilon)$ is an odd function in $\epsilon$. As a result, the first line of Eq. (5.6) reveals that the Majorana dot Green function $D_{b b}^{\mathrm{A}}\left(t, t^{\prime}\right)$ must be completely imaginary. This can be used to write

$$
\begin{aligned}
I_{c}(t) & =\frac{e g_{\perp}^{2}}{\hbar^{2}} \int_{-\infty}^{\infty} \mathrm{d} t^{\prime} \operatorname{Im}\left[-\frac{i}{v_{F}} \int_{-\infty}^{\infty} \frac{\mathrm{d} \epsilon}{2 \pi \hbar} f(\epsilon) e^{-\frac{i \epsilon}{\hbar}\left(t-t^{\prime}\right)+\frac{i e}{2 \hbar} \int_{t^{\prime}}^{t} \mathrm{~d} t^{\prime \prime} V\left(t^{\prime \prime}\right)} i D_{b b}^{\mathrm{A}}\left(t^{\prime}, t\right)\right] \\
& =\frac{e \Gamma}{2 \hbar} \operatorname{Im}\left[\int_{-\infty}^{\infty} \frac{\mathrm{d} \epsilon}{2 \pi \hbar} f(\epsilon) A(\epsilon, t)\right]
\end{aligned}
$$

with

$$
A(\epsilon, t) \equiv \int_{-\infty}^{\infty} \mathrm{d} t^{\prime} e^{-\frac{i \epsilon}{\hbar}\left(t-t^{\prime}\right)+\frac{i e}{2 \hbar} \int_{t^{\prime}}^{t} \mathrm{~d} t^{\prime \prime} V\left(t^{\prime \prime}\right)} D_{b b}^{\mathrm{A}}\left(t^{\prime}, t\right) .
$$

This equation is the most general expression for the charge current, only depending on the bias voltage and a Majorana component of the Green function on the dot. As such, it forms the basis for finding the charge 
current for any time-dependent bias voltage, the problem now having been reduced to finding the auxiliary function $A(\epsilon, t)$.

Since the bare dot Green function $\mathbf{D}_{0}$ has not been specified in the derivation of Eqs. (5.7) and (5.8), these results are even valid for more general on-site dot behaviour. However, we will restrict ourselves to the model at hand, where the bare on-site dot behaviour is fully determined by the magnetic field B. As is shown in Appendix A.2, the necessary Majorana dot Green function is given by

$$
\begin{aligned}
D_{b b}^{\mathrm{R} / \mathrm{A}}\left(t, t^{\prime}\right)= & \mp i \theta\left( \pm\left(t-t^{\prime}\right)\right) e^{\mp \frac{\Gamma}{2 \hbar}\left(t-t^{\prime}\right)}\left[\cosh \left(\frac{1}{2 \hbar} \sqrt{\Gamma^{2}-4 B^{2}}\left(t-t^{\prime}\right)\right)\right. \\
& \left.\mp \frac{\Gamma}{\sqrt{\Gamma^{2}-4 B^{2}}} \sinh \left(\frac{1}{2 \hbar} \sqrt{\Gamma^{2}-4 B^{2}}\left(t-t^{\prime}\right)\right)\right],
\end{aligned}
$$

while its Fourier transform is equal to

$$
D_{b b}^{\mathrm{R} / \mathrm{A}}(\epsilon)=\frac{\hbar \epsilon}{\epsilon(\epsilon \pm i \Gamma)-B^{2}} .
$$

Plugging this back into the general expression for the current, we now have an integral expression that solely depends on the voltage function $V(t)$.

Before applying the above framework, we note that the same methods can be used to calculate the charge current in presence of a channel asymmetry. Without explicitly showing the derivation, we point out that the expectation value of the modified current operator is given by

$$
\begin{aligned}
I_{c}(t)= & \frac{e}{2 \hbar} \operatorname{Im}\left[\int_{-\infty}^{\infty} \frac{\mathrm{d} \epsilon}{2 \pi \hbar} f(\epsilon)\left(\Gamma_{\perp} A_{\perp}(\epsilon, t)+\Gamma_{\Delta} A_{\Delta}(\epsilon, t)\right)\right] \\
& +\frac{e g_{\perp} g_{\Delta}}{2 \hbar^{2} v_{F}}\left(D_{b a}^{\mathrm{K}}(t, t)-D_{a b}^{\mathrm{K}}(t, t)\right)
\end{aligned}
$$

in absence of a magnetic field, where

$$
A_{\perp / \Delta}(\epsilon, t) \equiv \int_{-\infty}^{\infty} \mathrm{d} t^{\prime} e^{-\frac{i e}{\hbar}\left(t-t^{\prime}\right)+\frac{i e}{2 \hbar} \int_{t^{\prime}}^{t} \mathrm{~d} t^{\prime \prime} V\left(t^{\prime \prime}\right)} D_{b b / a a}^{\mathrm{A}}\left(t^{\prime}, t\right)
$$

and $\Gamma_{\perp / \Delta} \equiv 2 g_{\perp / \Delta}^{2} / \hbar v_{F} \equiv\left(J^{ \pm}\right)^{2} / 4 \pi a_{0} \hbar v_{F}$, using the parameters as they were defined in Sec. 3.5. The asymmetric case thus also requires calculating Keldysh components of the dot Green function, which can be done using

$$
\mathbf{D}^{\mathrm{K}}(t, t)=\int_{-\infty}^{\infty} \mathrm{d} t^{\prime} \int_{-\infty}^{\infty} \mathrm{d} t^{\prime \prime} \mathbf{D}^{\mathrm{R}}\left(t, t^{\prime}\right) \cdot \Sigma_{d}^{\mathrm{K}}\left(t^{\prime}, t^{\prime \prime}\right) \cdot \mathbf{D}^{\mathrm{A}}\left(t^{\prime \prime}, t\right),
$$


see Appendix A.3. When considering the full dot Green function, one should furthermore take into account that the inverse Green function from Eq. (4.26) now contains hybridization terms between the dot and the flavour modes as well (the latter being represented by $\mathbf{R}_{0}^{-1}$ ), complicating the propagator structure and significantly increasing the number of terms that have to be calculated. As such, although the methods for studying transport in the asymmetric case are indeed a straightforward generalization of the previous methods, the technical details become a lot more involved. It is for this reason that we only cover the symmetric case explicitly (where we use the presence of a magnetic field as a suitable alternative to channel asymmetries), and only occasionally comment on the asymmetric case.

\subsection{The dc solution}

We first consider dc, with $V(t)=V$. In this case the function $A(\epsilon, t)$ reduces to $D_{b b}^{\mathrm{A}}(\epsilon-e V / 2)$ (see also Appendix A.2). Using Eq. (5.6), we find

$$
I_{c}(t)=-\frac{i e \Gamma}{4 \hbar} \int_{-\infty}^{\infty} \frac{\mathrm{d} \epsilon}{2 \pi \hbar}\left[f\left(\epsilon+\frac{e V}{2}\right)-f\left(\epsilon-\frac{e V}{2}\right)\right] D_{b b}^{\mathrm{A}}(\epsilon) .
$$

The combination $f(\epsilon+e V / 2)-f(\epsilon-e V / 2)$ is even in $\epsilon$, so the odd part of $D_{b b}^{\mathrm{A}}(\epsilon)$ does not contribute to the overall integral. Furthermore, one can use the expression from Eq. 5.10 to show that the even part of $D_{b b}^{\mathrm{A}}(\epsilon)$ is exactly equal to the imaginary part. The above can thus be written as

$$
I_{c}(t)=-\frac{e \Gamma}{2} \int_{-\infty}^{\infty} \frac{\mathrm{d} \epsilon}{2 \pi \hbar}\left[n_{F}\left(\epsilon-\frac{e V}{2}\right)-n_{F}\left(\epsilon+\frac{e V}{2}\right)\right] \operatorname{Im}\left[\frac{\epsilon}{\epsilon(\epsilon+i \Gamma)-B^{2}}\right],
$$

where we also used $f(\epsilon+e V / 2)-f(\epsilon-e V / 2)=2\left(n_{F}(\epsilon-e V / 2)-n_{F}(\epsilon+e V / 2)\right)$, together with $\operatorname{Im}\left[D_{b b}^{\mathrm{A}}(\epsilon)\right]=-\operatorname{Im}\left[D_{b b}^{\mathrm{R}}(\epsilon)\right]$. Note that the latter object is simply $\pi$ times the spectral function corresponding to the $b$ Majorana fermion and that the expression is indeed completely independent of $t$. Moreover, Eq. (5.15) is in agreement with the known results for the anisotropic spin 2CK model from Ref. [13] :

\footnotetext{
${ }^{1}$ The details of the expression for the dc charge current differ from Ref. 13. The reason for this is that the charge and current operators of the $\mathrm{C} 2 \mathrm{CK}$ model only involve
} 
Our next goal is to evaluate the remaining integral. We do this by making use of the Matsubara representation of the Fermi-Dirac distribution,

$$
n_{F}(\epsilon)=\frac{1}{\beta} \sum_{n=-\infty}^{\infty} \frac{e^{i \omega_{n} 0^{+}}}{i \hbar \omega_{n}-\epsilon}, \quad \omega_{n} \equiv \frac{\pi(2 n+1)}{\hbar \beta},
$$

where $\omega_{n}$ are the fermionic Matsubara frequencies. As before, the chemical potential $\mu$ is absent from this expression due to our choice to measure all energies with respect to it. Plugging this back into Eq. 5.15) and splitting the sum into two parts, we find

$$
\begin{aligned}
I_{c}= & \frac{e \Gamma}{2 \pi \hbar \beta} \sum_{n=0}^{\infty} \operatorname{Re}\left[\int_{-\infty}^{\infty} \mathrm{d} \epsilon \frac{\Gamma \epsilon^{2}}{\epsilon^{4}+\left(\Gamma^{2}-2 B^{2}\right) \epsilon^{2}+B^{4}}\right. \\
& \left.\times\left(\frac{e^{i \omega_{n} 0^{+}}}{i \hbar \omega_{n}-(\epsilon-e V / 2)}-\frac{e^{i \omega_{n} 0^{+}}}{i \hbar \omega_{n}-(\epsilon+e V / 2)}\right)\right],
\end{aligned}
$$

where we used that the sum from $-\infty$ to -1 is simply the complex conjugate of the sum from 0 to $\infty$. The remaining integral can now be evaluated using contour integration. Closing the contour in the lower half of the imaginary plane and assuming $4 B^{2}<\Gamma^{2}$, the enclosed poles are located at

$$
\epsilon=-i \epsilon_{ \pm} \equiv-i \Gamma \sqrt{\frac{1}{2}-\left(\frac{B}{\Gamma}\right)^{2} \pm \sqrt{\frac{1}{4}-\left(\frac{B}{\Gamma}\right)^{2}}} .
$$

The residue of the diverging part of the integrand at these poles is given by

$$
\operatorname{Res}\left(\frac{\Gamma \epsilon^{2}}{\epsilon^{4}+\left(\Gamma^{2}-2 B^{2}\right) \epsilon^{2}+B^{4}},-i \epsilon_{ \pm}\right)= \pm \frac{i \epsilon_{ \pm}}{2 \Gamma \sqrt{1-4\left(\frac{B}{\Gamma}\right)^{2}}} .
$$

Using the residue theorem, we now find

$$
\begin{aligned}
I_{c}= & \frac{e}{2 \hbar \beta} \frac{1}{\sqrt{1-4\left(\frac{B}{\Gamma}\right)^{2}}} \sum_{\alpha= \pm} \alpha \epsilon_{\alpha} \\
& \times \sum_{n=0}^{\infty} \operatorname{Re}\left[\frac{1}{i \hbar \omega_{n}+\left(i \epsilon_{\alpha}+e V / 2\right)}-\frac{1}{i \hbar \omega_{n}+\left(i \epsilon_{\alpha}-e V / 2\right)}\right],
\end{aligned}
$$

pseudospin up, rather than both spin species. The most important difference is that the spin $2 \mathrm{CK}$ model does not support any charge transport for our choice of parameters (i.e. $J^{-}=0$ and $J_{\perp}^{L R}=0$ ), while the C2CK model does. Subsequent references to "known" results mostly refer to the spin $2 \mathrm{CK}$ model, taking into account that they cannot be applied to the $\mathrm{C} 2 \mathrm{CK}$ model before being rederived with the appropriate operators. 
where we discarded the factor $e^{i \omega_{n} 0^{+}}$. The latter is allowed because this factor only becomes important in the large- $n$ limit, while the rest of the summand scales with $n^{-2}$ and does therefore not contribute in this limit. To finish the calculation, we make use of the digamma function. This function is defined as the first derivative of the logarithm of the gamma function, and has the property that it commutes with complex conjugation:

$$
\Psi(z) \equiv \frac{\mathrm{d} \ln \Gamma(z)}{\mathrm{d} z}, \quad \Psi\left(z^{*}\right)=(\Psi(z))^{*} .
$$

For the purpose of transport calculations at non-zero temperatures, the most important property of the digamma function is that it can be represented by the following sum:

$$
\Psi(a)-\Psi(b)=(a-b) \sum_{n=0}^{\infty} \frac{1}{(n+a)(n+b)},
$$

where $\operatorname{Re}[a]>0$ and $\operatorname{Re}[b]>0$. More information about the digamma function, including a derivation of the latter identity, can be found in Appendix A.5. Using the above identity, it follows that

$$
\begin{aligned}
\sum_{n=0}^{\infty} \operatorname{Re} & {\left[\frac{1}{i \hbar \omega_{n}+\left(i \epsilon_{\alpha} \pm e V / 2\right)}\right] } \\
& =\left(\frac{\beta}{2 \pi}\right)^{2} \sum_{n=0}^{\infty} \frac{ \pm e V / 2}{\left(n+\frac{1}{2}+\frac{\epsilon_{\alpha} \mp i e V / 2}{2 \pi k_{B} T}\right)\left(n+\frac{1}{2}+\frac{\epsilon_{\alpha} \pm i e V / 2}{2 \pi k_{B} T}\right)} \\
& =\frac{i \beta}{4 \pi}\left[\Psi\left(\frac{1}{2}+\frac{\epsilon_{\alpha} \mp i e V / 2}{2 \pi k_{B} T}\right)-\Psi\left(\frac{1}{2}+\frac{\epsilon_{\alpha} \pm i e V / 2}{2 \pi k_{B} T}\right)\right] \\
& =\frac{\beta}{2 \pi} \operatorname{Im}\left[\Psi\left(\frac{1}{2}+\frac{\epsilon_{\alpha} \pm i e V / 2}{2 \pi k_{B} T}\right)\right] .
\end{aligned}
$$

This finally gives us the following exact expression for the dc current of the charge variant of the $2 \mathrm{CK}$ model at the EK point:

$$
\begin{aligned}
I_{c}= & \frac{e}{2 \pi \hbar} \frac{1}{\sqrt{1-4\left(\frac{B}{\Gamma}\right)^{2}}} \\
& \times\left(\epsilon_{+} \operatorname{Im}\left[\Psi\left(\frac{1}{2}+\frac{\epsilon_{+}+i e V / 2}{2 \pi k_{B} T}\right)\right]-\epsilon_{-} \operatorname{Im}\left[\Psi\left(\frac{1}{2}+\frac{\epsilon_{-}+i e V / 2}{2 \pi k_{B} T}\right)\right]\right) .
\end{aligned}
$$



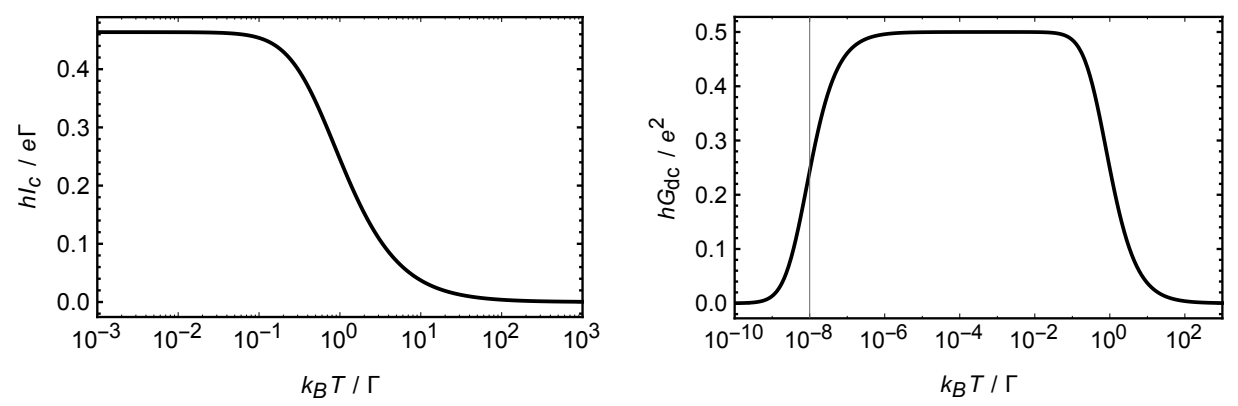

Figure 5.1: Dc electric transport of the charge variant of the $2 \mathrm{CK}$ model at the EK point. Left: dc current as a function of temperature for $\mathrm{eV} / \Gamma=1$ and $B^{2} / \Gamma^{2}=0$. Right: de differential conductance with $V \rightarrow 0$ and $B^{2} / \Gamma^{2}=10^{-8}$. The vertical line corresponds to the FL crossover temperature.

Having found the current as a function of the bias voltage, another interesting quantity to study is the differential conductance. For our purposes, we define it as

$$
G \equiv\left\langle\frac{\mathrm{d} I_{c}(t)}{\mathrm{d} V(t)}\right\rangle_{t}
$$

where $\langle\ldots\rangle_{t}$ denotes the time average. In the dc case, this gives us

$$
\begin{aligned}
G_{\mathrm{dc}}= & \frac{e^{2}}{4 \pi \hbar} \frac{1}{\sqrt{1-4\left(\frac{B}{\Gamma}\right)^{2}}}\left(\frac{\epsilon_{+}}{2 \pi k_{B} T} \operatorname{Re}\left[\psi^{(1)}\left(\frac{1}{2}+\frac{\epsilon_{+}+i e V / 2}{2 \pi k_{B} T}\right)\right]\right. \\
& \left.-\frac{\epsilon_{-}}{2 \pi k_{B} T} \operatorname{Re}\left[\psi^{(1)}\left(\frac{1}{2}+\frac{\epsilon_{-}+i e V / 2}{2 \pi k_{B} T}\right)\right]\right),
\end{aligned}
$$

where $\psi^{(1)}(z)$ is the trigamma function, i.e. the derivative of $\Psi(z)$. From here, the linear response conductance can be obtained by simply taking the limit $V \rightarrow 0$ :

$$
\begin{aligned}
G_{\mathrm{dc}} \stackrel{V \rightarrow 0}{ } & \frac{e^{2}}{4 \pi \hbar} \frac{1}{\sqrt{1-4\left(\frac{B}{\Gamma}\right)^{2}}} \\
& \times\left(\frac{\epsilon_{+}}{2 \pi k_{B} T} \psi^{(1)}\left(\frac{1}{2}+\frac{\epsilon_{+}}{2 \pi k_{B} T}\right)-\frac{\epsilon_{-}}{2 \pi k_{B} T} \psi^{(1)}\left(\frac{1}{2}+\frac{\epsilon_{-}}{2 \pi k_{B} T}\right)\right) .
\end{aligned}
$$

Fig. 5.1 shows examples of the dc current and conductance as functions of the temperature. Indeed, we witness the FL crossover as the temperature falls below a certain temperature scale $T^{*}$, in this case $k_{B} T^{*}=B^{2} / \Gamma$. 
As was explained in Sec. 3.6, the EK point results are only exact for the C2CK device if $T \ll T_{K}$. In order to take this limit, we first have to identify the Kondo scale. As can be seen in Fig. 5.1, the transition from the NFL region to the local moment region happens when the temperature scale becomes of the order of $\Gamma$, such that $k_{B} T_{K} \sim \Gamma$. This can be understood from the system's energy scales: the Kondo effect originates from the coupling between the dot and the leads, so the Kondo energy scale must be the energy scale corresponding to these interactions. Meanwhile, the FL crossover scale can be read off to be $k_{B} T^{*}=B^{2} / \Gamma$. In the limit $B^{2} \ll \Gamma^{2}$, i.e. when $T^{*} \ll T_{K}$, we have $\epsilon_{+} \rightarrow \Gamma \sim k_{B} T_{K}$ and $\epsilon_{-} \rightarrow B^{2} / \Gamma=k_{B} T^{*}$. Combining this with sending $T_{K} \rightarrow \infty$ and using the limit

$$
\lim _{x \rightarrow 0} \frac{1}{x} \psi^{(1)}\left(\frac{1}{2}+\frac{1}{x}\right)=1,
$$

we recover the known dc conductance [13, 35, 57, 58,

$$
G_{\mathrm{dc}}=\frac{e^{2}}{2 h}\left(1-\frac{T^{*}}{2 \pi T} \operatorname{Re}\left[\psi^{(1)}\left(\frac{1}{2}+\frac{T^{*}}{2 \pi T}+\frac{i e V}{4 \pi k_{B} T}\right)\right]\right) .
$$

This is the the main result of this section: it is the exact charge conductance of the C2CK model along the FL crossover, valid for the experimental setup ${ }^{2}$ One has to be careful, however, with using this result to make predictions involving experiments, as it requires both the temperature and the bias voltage to be much smaller than the Kondo scale. Nevertheless, it is worth mentioning that it is quite extraordinary for it to be possible to find such exact results for non-zero temperatures.

\subsection{The ac solution}

Next, we discuss the ac case, which is represented by the sinusoidal bias voltage $V(t)=V_{0}+\Delta V \cos \left(\omega_{0} t\right)$. The periodicity of the system makes it particularly suitable to be analysed using Floquet theory (analogous to

\footnotetext{
${ }^{2}$ Following the discussion from Sec. 3.4 the result from Eq. 5.29 is the same for both a magnetic field and a channel asymmetry. Indeed, using Eq. 5.11 and repeating this section's procedure, one finds that a channel asymmetry leads to the same dc conductance, but with $k_{B} T^{*}=\left(J_{\perp}^{L L}-J_{\perp}^{R R}\right)^{2} / 16 \pi a_{0} \hbar v_{F}$ instead of $k_{B} T^{*}=B^{2} / \Gamma$. It should furthermore be noted that these two explicit expressions for $T^{*}$ might not be particularly useful for practical purposes (e.g. for experiments). Instead, one can simply define $T^{*}$ as the temperature at which the zero bias dc conductance is equal to half its maximum value, where we use that $\left(1-\psi^{(1)}(1 / 2+1 / 2 \pi) / 2 \pi\right)=0.503 \ldots \approx 1 / 2$.
} 
Bloch theory for spatial periodicity), involving a discrete decomposition of $A(\epsilon, t)$ into frequency-dependent eigenstates. With the above sinusoidal bias voltage, this function is given by

$$
A(\epsilon, t)=\int_{-\infty}^{\infty} \mathrm{d} t^{\prime} e^{-\frac{i\left(\epsilon-e V_{0} / 2\right)}{\hbar}\left(t-t^{\prime}\right)+\frac{i e \Delta V}{2 \hbar} \int_{t^{\prime}}^{t} \mathrm{~d} t^{\prime \prime} \cos \left(\omega_{0} t^{\prime \prime}\right)} D_{b b}^{\mathrm{A}}\left(t^{\prime}, t\right) .
$$

The trick to evaluate this expression is to note that all terms of $D_{b b}^{\mathrm{A}}\left(t^{\prime}, t\right)$ are of the form $c \theta\left(t-t^{\prime}\right) e^{-z\left(t-t^{\prime}\right)}$, with $\operatorname{Re}[z]>0$. To find an analytic expression for the function $A(\epsilon, t)$, we consider the following sum [52], involving Bessel functions of the first kind $J_{n}(\alpha)$ (see also Appendix A.6):

$$
\sum_{n=-\infty}^{\infty} e^{-i n \omega t} J_{n}(\alpha)=e^{-i \alpha \sin (\omega t)}
$$

Using this identity, all terms of $A(\epsilon, t)$ are thus of the form

$$
\begin{aligned}
& e^{-\left(z+i\left(\epsilon-e V_{0} / 2\right) / \hbar\right) t+\frac{i e \Delta V}{2 \hbar \omega_{0}} \sin \left(\omega_{0} t\right)} \int_{-\infty}^{t} \mathrm{~d} t^{\prime} e^{\left(z+i\left(\epsilon-e V_{0} / 2\right) / \hbar\right) t^{\prime}-\frac{i e \Delta V}{2 \hbar \omega_{0}} \sin \left(\omega_{0} t^{\prime}\right)} \\
& =e^{-\left(z+i\left(\epsilon-e V_{0} / 2\right) / \hbar\right) t+\frac{i e \Delta V}{2 \hbar \omega_{0}} \sin \left(\omega_{0} t\right)} \sum_{n=-\infty}^{\infty} J_{n}\left(\frac{e \Delta V}{2 \hbar \omega_{0}}\right) \int_{-\infty}^{t} \mathrm{~d} t^{\prime} e^{\left(z+i\left(\epsilon-e V_{0} / 2-n \hbar \omega_{0}\right) / \hbar\right) t^{\prime}} \\
& =\sum_{n=-\infty}^{\infty} J_{n}\left(\frac{e \Delta V}{2 \hbar \omega_{0}}\right) \frac{e^{i\left(\frac{e \Delta V}{2 \hbar \omega_{0}} \sin \left(\omega_{0} t\right)-n \omega_{0} t\right)}}{z+i\left(\epsilon-e V_{0} / 2-n \hbar \omega_{0}\right) / \hbar} .
\end{aligned}
$$

In absence of $\Delta V$ (i.e. in the dc case), this same object would have read

$$
\frac{1}{z+i\left(\epsilon-e V_{0} / 2\right) / \hbar}=\sum_{n=-\infty}^{\infty} J_{n}\left(\frac{e \Delta V}{2 \hbar \omega_{0}}\right) \frac{e^{i\left(\frac{e \Delta V}{2 \hbar \omega_{0}} \sin \left(\omega_{0} t\right)-n \omega_{0} t\right)}}{z+i\left(\epsilon-e V_{0} / 2\right) / \hbar},
$$

where the right-hand side is completely independent of the dimensionless dummy parameter $e \Delta V / 2 \hbar \omega_{0}$. The dc case can therefore be generalized to the ac case by plugging in the above Bessel function and exponent, and subsequently writing $\epsilon \rightarrow \epsilon-n \hbar \omega_{0}$. Using that $A_{\mathrm{dc}}(\epsilon, t)=D_{b b}^{\mathrm{A}}\left(\epsilon-e V_{0} / 2\right)$, this procedure yields the following ac expression for $A(\epsilon, t)$ :

$$
A(\epsilon, t)=\sum_{n=-\infty}^{\infty} J_{n}\left(\frac{e \Delta V}{2 \hbar \omega_{0}}\right) e^{i\left(\frac{e \Delta V}{2 \hbar \omega_{0}} \sin \left(\omega_{0} t\right)-n \omega_{0} t\right)} D_{b b}^{\mathrm{A}}\left(\epsilon-\frac{e V_{0}}{2}-n \hbar \omega_{0}\right) .
$$


Returning to Eq. (5.7) and applying this result, we now find

$$
\begin{aligned}
I_{c}(t)= & \frac{e \Gamma}{2 \hbar} \operatorname{Im}\left[e^{i \frac{e \Delta V}{2 \hbar \omega_{0}} \sin \left(\omega_{0} t\right)} \sum_{n=-\infty}^{\infty} e^{-i n \omega_{0} t} J_{n}\left(\frac{e \Delta V}{2 \hbar \omega_{0}}\right)\right. \\
& \left.\times \int_{-\infty}^{\infty} \frac{\mathrm{d} \epsilon}{2 \pi \hbar} f\left(\epsilon+\frac{e V_{0}}{2}+n \hbar \omega_{0}\right) D_{b b}^{\mathrm{A}}(\epsilon)\right] .
\end{aligned}
$$

To make sense of this expression, we furthermore replace $f\left(\epsilon+e V_{0} / 2+n \hbar \omega_{0}\right)$ by $f\left(\epsilon+e V_{0} / 2+n \hbar \omega_{0}\right)-f(\epsilon)$. We can do this because the added term is odd in $\epsilon$, while not containing any $n$-dependence. Lacking any $n$-dependence, all the prefactors in front of the integral of this additional term are together equal to 1 , so this term is proportional to the integral over $f(\epsilon)$ (an odd function) times the imaginary part of $D_{b b}^{\mathrm{A}}(\epsilon)$. As we discussed before, the latter is even, so the integral vanishes and this additional term is therefore equal to zero. Applying this trick together with some of the observations also used for the dc case, we obtain

$$
\begin{aligned}
I_{c}(t)= & e \Gamma \operatorname{Im}\left[e^{i \frac{e \Delta V}{2 \hbar \omega_{0}} \sin \left(\omega_{0} t\right)} \sum_{n=-\infty}^{\infty} e^{-i n \omega_{0} t} J_{n}\left(\frac{e \Delta V}{2 \hbar \omega_{0}}\right)\right. \\
& \left.\times \int_{-\infty}^{\infty} \frac{\mathrm{d} \epsilon}{2 \pi \hbar}\left[n_{F}(\epsilon)-n_{F}\left(\epsilon-\frac{e V_{0}}{2}-n \hbar \omega_{0}\right)\right] \frac{\epsilon}{\epsilon(\epsilon+i \Gamma)-B^{2}}\right],
\end{aligned}
$$

agreeing with the ac results for the anisotropic spin 2CK model from Ref. [12].

The problem with further evaluation of the ac current is that the sum over the Bessel functions cannot be performed exactly. In order to still extract any of the desired information from Eq. (5.36), we will write it in terms of its Fourier components, using the convention

$$
I_{c}(t)=\sum_{n=-\infty}^{\infty} I_{n} e^{-i n \omega_{0} t} \quad \Longleftrightarrow \quad I_{n}=\frac{1}{T} \int_{-T / 2}^{T / 2} \mathrm{~d} t I_{c}(t) e^{i n \omega_{0} t},
$$

where $T$ is the period of the current, and $\omega_{0}=2 \pi / T$ is the corresponding frequency (which is the same as our previous $\omega_{0}$ due to the periodicity of 
the underlying Hamiltonian). Using Eq. (5.31), the Fourier components of the time-dependences of the current can be written as

$$
\begin{aligned}
\frac{1}{T} \int_{-T / 2}^{T / 2} \mathrm{~d} t e^{i\left(n \mp n^{\prime}\right) \omega_{0} t \pm \frac{i e \Delta V}{2 \hbar \omega_{0}} \sin \left(\omega_{0} t\right)} & =\frac{1}{T} \sum_{m=-\infty}^{\infty} \int_{-T / 2}^{T / 2} \mathrm{~d} t e^{i\left( \pm m+n \mp n^{\prime}\right) \omega_{0} t} J_{m}\left(\frac{e \Delta V}{2 \hbar \omega_{0}}\right) \\
& =\sum_{m=-\infty}^{\infty} \delta_{ \pm m,-n \pm n^{\prime}} J_{m}\left(\frac{e \Delta V}{2 \hbar \omega_{0}}\right)=J_{n^{\prime} \mp n}\left(\frac{e \Delta V}{2 \hbar \omega_{0}}\right) .
\end{aligned}
$$

Additionally, we use $\operatorname{Im}[f(t) z]=\left(f(t) z-(f(t) z)^{*}\right) / 2 i$ to find that the Fourier components of a function of this form are simply $\left(f_{n} z-\left(f^{*}\right)_{n} z^{*}\right) / 2 i$, where $\left(f^{*}\right)_{n}$ are the Fourier components of the complex conjugate of $f(t)$. The Fourier components of the current are therefore given by

$$
\begin{aligned}
I_{n}= & -\frac{i e \Gamma}{2} \sum_{n^{\prime}=-\infty}^{\infty} J_{n^{\prime}}\left(\frac{e \Delta V}{2 \hbar \omega_{0}}\right) \int_{-\infty}^{\infty} \frac{\mathrm{d} \epsilon}{2 \pi \hbar}\left[n_{F}(\epsilon)-n_{F}\left(\epsilon-\frac{e V_{0}}{2}-n^{\prime} \hbar \omega_{0}\right)\right] \\
& \times\left(J_{n^{\prime}-n}\left(\frac{e \Delta V}{2 \hbar \omega_{0}}\right) \frac{\epsilon}{\epsilon(\epsilon+i \Gamma)-B^{2}}-J_{n^{\prime}+n}\left(\frac{e \Delta V}{2 \hbar \omega_{0}}\right) \frac{\epsilon}{\epsilon(\epsilon-i \Gamma)-B^{2}}\right) \\
= & \frac{i e \Gamma}{2} \sum_{n^{\prime}=-\infty}^{\infty} J_{n^{\prime}}\left(\frac{e \Delta V}{2 \hbar \omega_{0}}\right) J_{n^{\prime}+n}\left(\frac{e \Delta V}{2 \hbar \omega_{0}}\right) \int_{-\infty}^{\infty} \frac{\mathrm{d} \epsilon}{2 \pi \hbar}\left(\frac{2 i \Gamma \epsilon^{2} n_{F}(\epsilon)}{\epsilon^{4}+\left(\Gamma^{2}-2 B^{2}\right) \epsilon^{2}+B^{4}}\right. \\
& \left.-\frac{\epsilon n_{F}\left(\epsilon-e V_{0} / 2-n^{\prime} \hbar \omega_{0}\right)}{\epsilon(\epsilon-i \Gamma)-B^{2}}+\frac{\epsilon n_{F}\left(\epsilon-e V_{0} / 2-\left(n^{\prime}+n\right) \hbar \omega_{0}\right)}{\epsilon(\epsilon+i \Gamma)-B^{2}}\right) .
\end{aligned}
$$

While it is possible to fully evaluate the remaining integral, it is quite cumbersome and not very insightful, as one would still be left with sums that cannot be solved exactly. We will therefore not perform the calculation here (the interested reader can find further evaluation of the current for $B=0$ in Appendix A.7), and instead we will focus on the differential conductance. Nevertheless, a plot of the resulting current at a point residing on the FL crossover line is shown in the left panel of Fig. 5.2, illustrating the dynamics of the system.

Let us return to the previous definition of the differential conductance from Eq. (5.25). To keep things concise, we now set the constant offset $V_{0}$ to zero and only consider the small- $\Delta V$ limit. In other words: we restrict ourselves to the linear response regime, specifically considering the linear susceptibility of the current to a strictly ac bias voltage, evaluated in absence 

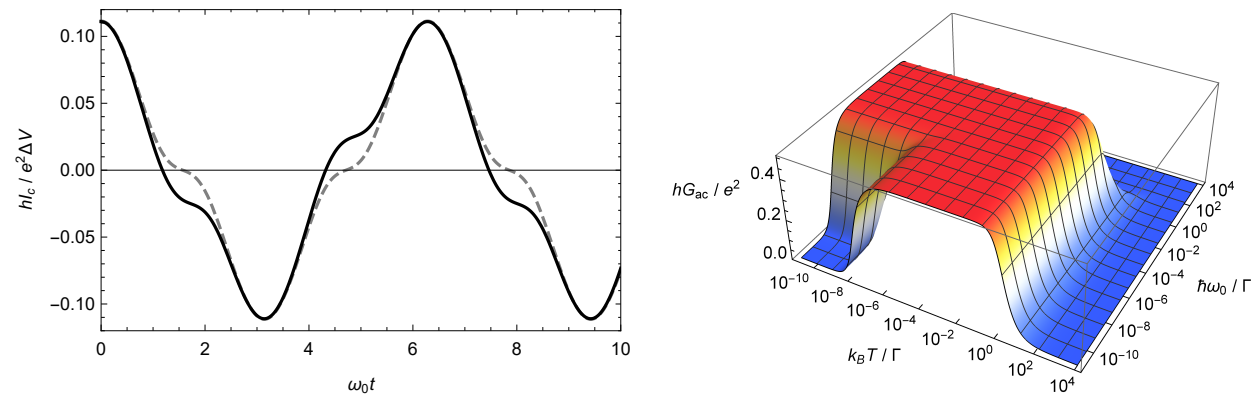

Figure 5.2: Ac electric transport of the charge variant of the $2 \mathrm{CK}$ model at the EK point. Left: example of the ac current (solid) versus its adiabatic limit $I_{c, \mathrm{dc}}(V(t))$ (dashed) as a function of time, with $T_{K} \rightarrow \infty, V_{0}=0, T / T^{*}=\hbar \omega_{0} / k_{B} T^{*}=0.1$ and $e \Delta V / k_{B} T^{*}=2$. Note that this point lies on the FL crossover line, such that this current profile is also applicable to the $\mathrm{C} 2 \mathrm{CK}$ device following the discussion from Sec.3.6 Right: ac differential conductance in the $V \rightarrow 0$ limit, with $T^{*} / T_{K}=10^{-8}$.

of a bias voltage. First, we expand the current in terms of $\Delta V$. We do this by employing the following expansions (see Appendix A.6):

$$
J_{0}(\alpha)=1+\mathcal{O}\left(\alpha^{2}\right), \quad J_{n \neq 0}(\alpha)=\frac{(\operatorname{sgn}(n))^{|n|}}{|n| !}\left(\frac{\alpha}{2}\right)^{|n|}+\mathcal{O}\left(\alpha^{|n|+2}\right) .
$$

Plugging these back into Eq. (5.39), it immediately follows that

$$
I_{n} \propto\left(\frac{e \Delta V}{2 \hbar \omega_{0}}\right)^{|n|}+\mathcal{O}\left(\left(e \Delta V / 2 \hbar \omega_{0}\right)^{|n|+2}\right) .
$$

The full time-dependent current can therefore be written as

$$
\begin{aligned}
I_{c}(t) & =I_{0}+I_{1} e^{-i \omega_{0} t}+I_{1}^{*} e^{i \omega_{0} t}+\mathcal{O}\left(\left(e \Delta V / 2 \hbar \omega_{0}\right)^{2}\right) \\
& =I_{0}+2 \operatorname{Re}\left[I_{1}\right] \cos \left(\omega_{0} t\right)+2 \operatorname{Im}\left[I_{1}\right] \sin \left(\omega_{0} t\right)+\mathcal{O}\left(\left(e \Delta V / 2 \hbar \omega_{0}\right)^{2}\right),
\end{aligned}
$$

where we used the reality condition for the current $I_{c}(t)$ to write $I_{-1}=I_{1}^{*}$. From here, we can calculate the linear response differential conductance:

$$
\begin{aligned}
G_{\mathrm{ac}} & =\left\langle\frac{\partial I_{c}(t)}{\partial t} / \frac{\partial V(t)}{\partial t}\right\rangle_{t} \\
& =\frac{2 \operatorname{Re}\left[I_{1}\right]}{\Delta V}-\frac{2 \operatorname{Im}\left[I_{1}\right]}{\Delta V}\left\langle\frac{\cos \left(\omega_{0} t\right)}{\sin \left(\omega_{0} t\right)}\right\rangle_{t}{ }_{t}^{0}
\end{aligned}
$$


Next, we expand the Fourier component $I_{1}$, using

$$
\begin{aligned}
& J_{n}(\alpha) J_{n+1}(\alpha) \\
& \quad=\left(\delta_{n, 0}+\frac{\alpha}{2}\left(\delta_{n, 1}-\delta_{n,-1}\right)+\mathcal{O}\left(\alpha^{2}\right)\right)\left(\delta_{n,-1}+\frac{\alpha}{2}\left(\delta_{n, 0}-\delta_{n,-2}\right)+\mathcal{O}\left(\alpha^{2}\right)\right) \\
& \quad=\frac{\alpha}{2}\left(\delta_{n, 0}-\delta_{n,-1}\right)+\mathcal{O}\left(\alpha^{2}\right) .
\end{aligned}
$$

Combining all of the above and carefully simplifying the result, we obtain the following linear response differential conductance of the $\mathrm{C} 2 \mathrm{CK}$ model at the EK point due to a purely ac bias voltage:

$$
\begin{aligned}
G_{\mathrm{ac}}= & \frac{e^{2} \Gamma}{4 \hbar \omega_{0}} \operatorname{Im}\left[\int _ { - \infty } ^ { \infty } \frac { \mathrm { d } \epsilon } { 2 \pi \hbar } \left(\frac{\epsilon n_{F}\left(\epsilon-\hbar \omega_{0}\right)}{\epsilon(\epsilon-i \Gamma)-B^{2}}+\frac{\epsilon n_{F}\left(\epsilon+\hbar \omega_{0}\right)}{\epsilon(\epsilon+i \Gamma)-B^{2}}\right.\right. \\
& \left.\left.-\frac{\epsilon n_{F}(\epsilon)}{\epsilon(\epsilon-i \Gamma)-B^{2}}-\frac{\epsilon n_{F}(\epsilon)}{\epsilon(\epsilon+i \Gamma)-B^{2}}\right)\right] \\
= & \frac{e^{2} \Gamma}{4 \hbar \omega_{0}} \int_{-\infty}^{\infty} \frac{\mathrm{d} \epsilon}{2 \pi \hbar}\left[n_{F}\left(\epsilon-\hbar \omega_{0}\right)-n_{F}\left(\epsilon+\hbar \omega_{0}\right)\right] \frac{\Gamma \epsilon^{2}}{\epsilon^{4}+\left(\Gamma^{2}-2 B^{2}\right) \epsilon^{2}+B^{4}} .
\end{aligned}
$$

This final integral is essentially the same as the one we already solved when discussing the dc current. Applying this dc result, the differential conductance finally becomes

$$
\begin{aligned}
G_{\mathrm{ac}}= & \frac{e^{2}}{4 \pi \hbar^{2} \omega_{0}} \frac{1}{\sqrt{1-4\left(\frac{B}{\Gamma}\right)^{2}}} \\
& \times\left(\epsilon_{+} \operatorname{Im}\left[\Psi\left(\frac{1}{2}+\frac{\epsilon_{+}+i \hbar \omega_{0}}{2 \pi k_{B} T}\right)\right]-\epsilon_{-} \operatorname{Im}\left[\Psi\left(\frac{1}{2}+\frac{\epsilon_{-}+i \hbar \omega_{0}}{2 \pi k_{B} T}\right)\right]\right) .
\end{aligned}
$$

It is worth noting that the dc limit $\omega_{0} \rightarrow 0$ of this expression is indeed equal to Eq. 5.27). Moreover, as is shown in the right panel of Fig. 5.2, the temperature and frequency dependences of the conductance are very similar to each other. The reason for this is that any energy scale can play the role of temperature in order for Kondo physics to occur, such that introducing an energy scale to the system by means of a driving voltage has the same effect as thermal fluctuations. 
Finally, we again take the limit $T_{K} \rightarrow \infty$, using the same identification of temperature scales as in the previous section. Using the asymptotic series $\Psi(z)=\ln z+\mathcal{O}(1 / z)$, we obtain the following expression for the linear response ac conductance along the FL crossover:

$$
G_{\mathrm{ac}}=\frac{e^{2}}{2 h}\left(1-\frac{k_{B} T^{*}}{\hbar \omega_{0}} \operatorname{Im}\left[\Psi\left(\frac{1}{2}+\frac{T^{*}}{2 \pi T}+\frac{i \hbar \omega_{0}}{2 \pi k_{B} T}\right)\right]\right) .
$$

In the limit $\Delta V \rightarrow 0$ and tuning $T_{K}$ to be much larger than all other energy scales, this expression remains exact in the context of the $\mathrm{C} 2 \mathrm{CK}$ device.

\subsection{Relaxation to a new steady state}

Our third and final example is a sudden quantum quench at $t=0$, where the bias voltage jumps from $V_{0}$ to $V_{0}+\Delta V$. Explicitly, the bias voltage is given by $V(t)=V_{0}+\Delta V \theta(t)$. We will use this voltage profile to study the relaxation of the current to a new steady state. Since the general calculations involving $T \neq 0$ are rather cumbersome while not revealing any particularly interesting additional behaviour, we restrict the present discussion to the NFL fixed point. The time-dependent electrical current corresponding to this quantum critical point is obtained by setting $B=0$ and $T=0$ in the EK point calculations, then taking the limit $e V(t) / \Gamma \rightarrow 0$.

Given a zero magnetic field, the advanced Majorana Green function of interest reduces to

$$
\left.D_{b b}^{\mathrm{A}}\left(t^{\prime}, t\right)\right|_{B=0}=i \theta\left(t-t^{\prime}\right) e^{-\frac{\Gamma}{\hbar}\left(t-t^{\prime}\right)},
$$

greatly simplifying the expression for $A(\epsilon, t)$ from Eq. (5.8). In order to calculate $A(\epsilon, t)$, we first perform the integral over the Heaviside step function:

$$
\int_{t^{\prime}}^{t} \mathrm{~d} t^{\prime \prime} \theta\left(t^{\prime \prime}\right)=\left(t-t^{\prime} \theta\left(t^{\prime}\right)\right) \theta\left(t-t^{\prime}\right) \theta(t)-\left(t^{\prime}-t \theta(t)\right) \theta\left(t^{\prime}-t\right) \theta\left(t^{\prime}\right) .
$$

The second term of the right-hand side does not contribute, because $t^{\prime} \leq t$ in the expression for $A(\epsilon, t)$. Moreover, the first term vanishes when $t<0$, such that $A(\epsilon, t<0)$ is equal to its initial dc value. On the other, the above integral effectively becomes $\left(t-t^{\prime} \theta\left(t^{\prime}\right)\right)$ when $t>0$. We thus find

$$
\begin{aligned}
A(\epsilon, t)= & i \hbar\left(\frac{1}{\Gamma+i(\epsilon-e V(t) / 2)}+\theta(t) e^{-\frac{\Gamma+i\left(\epsilon-e\left(V_{0}+\Delta V\right) / 2\right)}{\hbar} t}\right. \\
& \left.\times\left(\frac{1}{\Gamma+i\left(\epsilon-e V_{0} / 2\right)}-\frac{1}{\Gamma+i\left(\epsilon-e\left(V_{0}+\Delta V\right) / 2\right)}\right)\right) .
\end{aligned}
$$


Referring back to Eq. (5.7) and using that $f(\epsilon)=1-2 \theta(-\epsilon)$ in the limit $T \rightarrow 0$, we only have to calculate the integral

$$
\operatorname{Im}\left[\int_{-\infty}^{a} \frac{\mathrm{d} \epsilon}{2 \pi \hbar} A(\epsilon, t)\right]
$$

with $a=0$ and $a \rightarrow \infty$. For the first term of Eq. (5.50), the required integral is straightforward to evaluate:

$$
\begin{aligned}
\operatorname{Im}\left[\int_{-\infty}^{a} \frac{\mathrm{d} \epsilon}{2 \pi \hbar} \frac{i \hbar}{\Gamma+i(\epsilon-e V(t) / 2)}\right] & =\int_{-\infty}^{a} \frac{\mathrm{d} \epsilon}{2 \pi} \frac{\Gamma}{\Gamma^{2}+(\epsilon-e V(t) / 2)^{2}} \\
& =\frac{1}{2 \pi}\left(\frac{\pi}{2}+\arctan \left(\frac{2 a-e V(t)}{2 \Gamma}\right)\right) \\
& = \begin{cases}\frac{1}{2 \pi}\left(\frac{\pi}{2}-\arctan \left(\frac{e V(t)}{2 \Gamma}\right)\right), & a=0, \\
\frac{1}{2}, & a \rightarrow \infty .\end{cases}
\end{aligned}
$$

For the other terms, we define $x_{\epsilon} \equiv\left(i \Gamma-\left(\epsilon-e\left(V_{0}+\Delta V\right) / 2\right)\right) t / \hbar$ and integrate by substitution, using the variable $y \equiv x_{\epsilon} / x_{a}$ :

$$
\begin{aligned}
& \int_{-\infty}^{a} \frac{\mathrm{d} \epsilon}{2 \pi \hbar} \frac{e^{-\frac{\Gamma+i\left(\epsilon-e\left(V_{0}+\Delta V\right) / 2\right)}{\hbar} t}}{\Gamma+i\left(\epsilon-e\left(V_{0}+\Delta V\right) / 2\right)} \\
& =i \int_{1}^{\infty} \frac{\mathrm{d} y}{2 \pi \hbar} \frac{e^{i x_{a} y}}{y}=\frac{i}{2 \pi \hbar} E_{1}\left(-i x_{a}\right)=\frac{i}{2 \pi \hbar} E_{1}\left(\frac{\Gamma+i\left(a-e\left(V_{0}+\Delta V\right) / 2\right)}{\hbar} t\right),
\end{aligned}
$$

where we used that $\Gamma t / \hbar \geq 0$ for all terms of this forms to introduce the exponential integral,

$$
E_{1}(z) \equiv \int_{1}^{\infty} \mathrm{d} y \frac{e^{-z y}}{y}, \quad \operatorname{Re}[z] \geq 0 .
$$

Similarly, we find

$$
\int_{-\infty}^{a} \frac{\mathrm{d} \epsilon}{2 \pi \hbar} \frac{e^{-\frac{\Gamma+i\left(\epsilon-e\left(V_{0}+\Delta V\right) / 2\right)}{\hbar} t}}{\Gamma+i\left(\epsilon-e V_{0} / 2\right)}=\frac{i}{2 \pi \hbar} e^{\frac{i e \Delta V}{2 \hbar} t} E_{1}\left(\frac{\Gamma+i\left(a-e V_{0} / 2\right)}{\hbar} t\right) .
$$



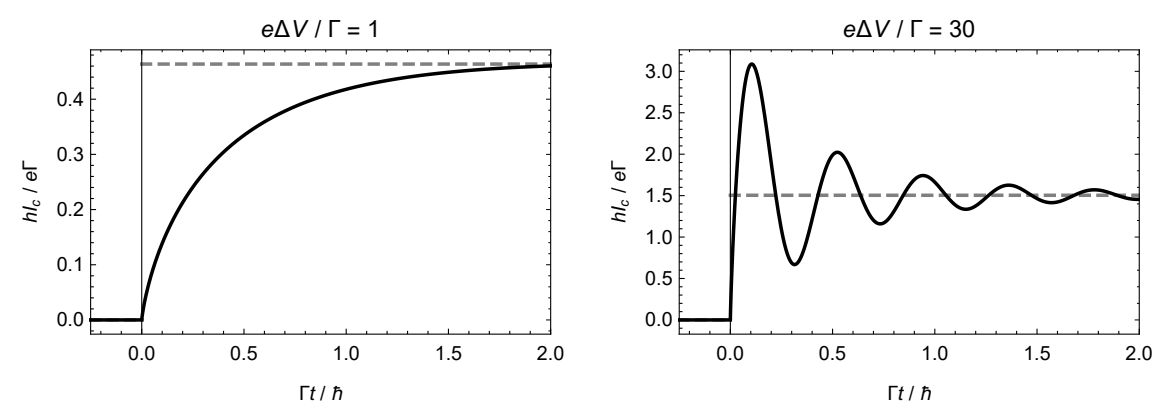

Figure 5.3: Electrical current of the charge variant of the $2 \mathrm{CK}$ model at the EK point due to a sudden quench in the bias voltage, with $B=0$ and $T=0$. In these examples, the initial bias voltage is zero, which then jumps to a non-zero value $\Delta V$ at $t=0$. The dashed lines denote the dc currents associated with the instantaneous bias voltage, i.e. the steady state solutions. Note that these plots are not applicable to the $\mathrm{C} 2 \mathrm{CK}$ device due to the fact that $e \Delta V / \Gamma$ does not go to zero.

We furthermore use the asymptotic series of the exponential integral (which can be found by integrating Eq. (5.54) by parts),

$$
E_{1}(z)=\frac{e^{-z}}{z}\left(1+\mathcal{O}\left(\frac{1}{z}\right)\right)
$$

to see that both of the above exponential integrals go to zero as $a \rightarrow \infty$. Plugging everything back into Eq. (5.7), we find the exact charge current at the EK point:

$$
\begin{aligned}
I_{c}(t)= & \frac{e \Gamma}{2 \pi \hbar}\left(\arctan \left(\frac{e V(t)}{2 \Gamma}\right)\right. \\
& \left.-\theta(t) \operatorname{Im}\left[E_{1}\left(\frac{\Gamma-i e\left(V_{0}+\Delta V\right) / 2}{\hbar} t\right)-e^{\frac{i e \Delta V}{2 \hbar} t} E_{1}\left(\frac{\Gamma-i e V_{0} / 2}{\hbar} t\right)\right]\right) .
\end{aligned}
$$

Note that everything except the first term vanishes if $\Delta V=0$, such that we indeed recover the zero temperature limit of the dc solution from Eq. (5.24). Moreover, the current also reduces to the dc solution when $t \rightarrow \infty$, but with the new bias voltage $V_{0}+\Delta V$. Figs. 5.3 and 5.4 show a couple of examples of the current as a function of the dimensionless time $\Gamma t / \hbar$.

The current exhibits several features. (i) As is illustrated in Fig. 5.3, the current is constant before the quantum quench disturbs the system at $t=0$, 


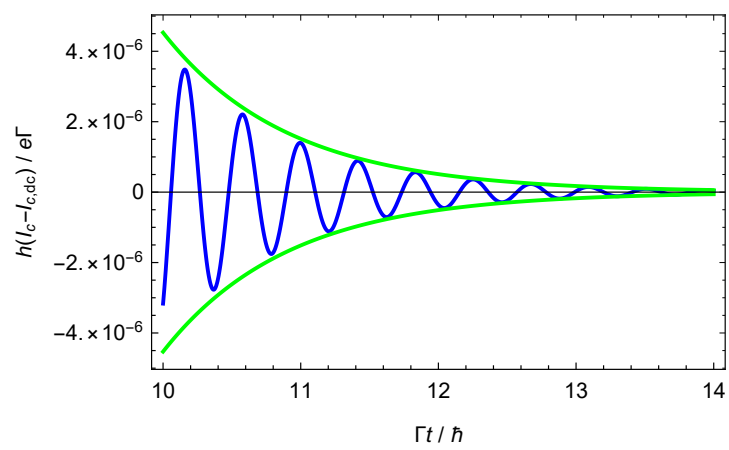

Figure 5.4: Oscillations of the current about its new steady state solution (blue) versus its approximated envelope (green) for $\Gamma t / \hbar \gg 1, B=0$ and $T=0$, calculated using Eqs. (5.57) and (5.59). The bias voltage is given by $V_{0}=0$ and $e \Delta V / \Gamma=30$.

after which it slowly moves towards its new steady state value. The new steady state is only completely reached as $t \rightarrow \infty$. (ii) The behaviour is very similar to that of a damped harmonic oscillator, with the system being either underdamped, critically damped or overdamped, depending on the value of $e\left(V_{0}+\Delta V\right) / \Gamma$. (iii) The oscillations of the current become larger and faster for increasing $e\left(V_{0}+\Delta V\right) / \Gamma$. The reason for this is that the tunnelling rate from the left to the right lead increases with $V_{0}+\Delta V$, resulting in a faster reaction of the current to deviations from the steady state. This increase in the tunnelling rate furthermore causes the current to increasingly overshoot its new desired value, leading to a larger amplitude.

It is also worth considering the asymptotic behaviour of the current, and in particular the relaxation of the current towards the new steady state. To do so, we use the same asymptotic series as before (see Eq. (5.56) ) to find

$$
\begin{aligned}
I_{c}(t) \stackrel{\Gamma t \gg \hbar}{\approx} & \frac{e \Gamma}{2 \pi \hbar}\left(\arctan \left(\frac{e\left(V_{0}+\Delta V\right)}{2 \Gamma}\right)\right. \\
& \left.\quad-\frac{e^{-\frac{\Gamma}{\hbar} t}}{t} \operatorname{Im}\left[\left(\frac{\hbar}{\Gamma-i e\left(V_{0}+\Delta V\right) / 2}-\frac{\hbar}{\Gamma-i e V_{0} / 2}\right) e^{\frac{i e\left(V_{0}+\Delta V\right)}{2 \hbar} t}\right]\right) .
\end{aligned}
$$

The convenient aspect of this expression is that all oscillatory behaviour is contained in the final (complex) exponential. The envelope of this oscillatory function, which contains the information about the decay towards the new steady state, can be calculated by using the fact that the amplitude of an 
oscillatory function $z e^{i \theta}$ is simply the absolute value $|z|$. For large $t$, the envelope of the current is thus given by

$$
\begin{aligned}
I_{c, \mathrm{env}}(t) \stackrel{\Gamma t \gg \hbar}{ } I_{c, \mathrm{dc}} \pm \frac{e \Gamma}{2 \pi \hbar}\left|\frac{\hbar}{\Gamma-i e\left(V_{0}+\Delta V\right) / 2}-\frac{\hbar}{\Gamma-i e V_{0} / 2}\right| \frac{e^{-\frac{\Gamma}{\hbar} t}}{t} \\
\quad=I_{c, \mathrm{dc}} \pm \frac{e^{2} \Gamma \Delta V}{4 \pi \sqrt{\left(\Gamma^{2}+(e V / 2)^{2}\right)\left(\Gamma^{2}+(e(V+\Delta V) / 2)^{2}\right)}} \frac{e^{-\frac{\Gamma}{\hbar} t}}{t} .
\end{aligned}
$$

Fig. 5.4 shows an example of the exact current at the EK point together with the approximated envelope for large $t$, and confirms the validity of the above approximation. We conclude that the relaxation of the current towards its new steady state solution is governed by the factor $e^{-\Gamma t / \hbar} / t$, with $\hbar / \Gamma$ being the associated relaxation time scale.

Since this section's results correspond to $B=0$ and $T=0$, the only remaining energy scales are $e V(t)$ and $\Gamma \sim k_{B} T_{K}$. The NFL fixed point can therefore be reached by taking the limit $e V(t) / \Gamma \rightarrow 0$. Using this limit, we find the following asymptotic expression for the charge current of the $\mathrm{C} 2 \mathrm{CK}$ device at the NFL fixed point:

$$
I_{c}(t) \stackrel{\Gamma t \gg \hbar}{\approx} \frac{e^{2} \Delta V}{2 h}\left(1+\frac{V_{0}}{\Delta V}-\frac{e^{-\Gamma t / \hbar}}{\Gamma t / \hbar} \cos \left(\frac{e\left(V_{0}+\Delta V\right)}{2 \hbar} t\right)\right) .
$$

From this expression it is immediately clear that the two remaining energy scales play distinct roles: the Kondo scale determines the relaxation time $t_{\text {rel }}$ through $t_{\text {rel }}=\hbar / \Gamma \sim \hbar / k_{B} T_{K}$, while the oscillations about the steady state solution have a period $t_{\text {osc }}$ that only depends on the driving energy scale through $t_{\mathrm{osc}}=2 h / e\left(V_{0}+\Delta V\right)$. However, we observe that $t_{\mathrm{rel}} \ll t_{\mathrm{osc}}$ at the NFL fixed point, such that the oscillations are very slow compared to the damping. The system is thus strongly overdamped, in the sense that the deviations of the experimentally observable current from its new steady state value will decay exponentially, without any apparent oscillations.

To summarize, we have seen how the conductance along the FL crossover of the $\mathrm{C} 2 \mathrm{CK}$ model can in principle be calculated for any time-dependent bias voltage, explicitly performing the calculations for three specific examples. We conclude this chapter by noting that the framework developed in this chapter allows for many more exact solutions, for example transport driven by a step-like modulation or a Dirac comb. 



\section{Chapter 6}

\section{Linear susceptibilities of the charge two-channel Kondo model}

So far, we have seen how electric transport in the $\mathrm{C} 2 \mathrm{CK}$ model can be treated exactly using Keldysh techniques. The main goal of this chapter is to find the differential conductance corresponding to heat transport as well. Contrary to the case of a bias voltage, fast changes in the temperature gradient are not realistic, so we will not consider any time-dependences in the temperature gradient beyond the dc solution. Moreover, due to the complexity of the heat current operator we will focus only on the NFL fixed point rather than the full FL crossover. However, as discussed in Sec. 4.2.5, even with these restrictions the strategy from the previous chapter cannot be used to find the heat current due to a temperature gradient between the leads, motivating the use of linear response theory.

Our starting point is the set of ingredients from Sec. 4.2, in particular the Kubo formula from Eq. 4.56). Referring back to the current operators from Eqs. 4.24 and 4.25), usage of the Kubo formula requires calculating four-, six- and eight-point functions. Crucially, all expectation values are taken in absence of a potential gradient and will be evaluated at the EK point, so they are all non-interacting. As a result, Wick's theorem can be applied to write all $2 n$-point functions in terms of equilibrium propagators. We will now use this observation to work out the dc linear response susceptibilities corresponding to both the charge current (along the FL crossover) and the heat current (at the NFL fixed point) of the $\mathrm{C} 2 \mathrm{CK}$ model.

\subsection{Charge transport}

We first apply linear response theory to charge transport, serving as a pedagogical example before moving on to the much more involved case of heat transport. Referring back to Eqs. 4.24 and 4.58), one can immediately write down the required four-point function. For four-point functions of 
Grassmann variables, Wick's theorem reads

$$
\langle a b c d\rangle_{0}=\langle a b\rangle_{0}\langle c d\rangle_{0}+\langle a d\rangle_{0}\langle b c\rangle_{0}-\langle a c\rangle_{0}\langle b d\rangle_{0},
$$

where $\langle\ldots\rangle_{0}$ again refers to the expectation value in absence of a bias. For the four-point function of interest,

$$
\begin{aligned}
C_{c}^{\tau}(\tau>0)= & \frac{e^{2} g_{\perp}^{2}}{4 \hbar^{2} L} \sum_{k, k^{\prime}}\left\langle\left(\psi_{s f, k}^{\dagger}(\tau)-\psi_{s f, k}(\tau)\right)\left(d^{\dagger}(\tau)-d(\tau)\right)\right. \\
& \left.\times\left(\psi_{s f, k^{\prime}}^{\dagger}(0)-\psi_{s f, k^{\prime}}(0)\right)\left(d^{\dagger}(0)-d(0)\right)\right\rangle_{0},
\end{aligned}
$$

the summand can thus be expressed as

$$
\begin{aligned}
& \left\langle\left(\psi_{s f, k}^{\dagger}(\tau)-\psi_{s f, k}(\tau)\right)\left(d^{\dagger}(\tau)-d(\tau)\right)\right\rangle_{0}\left\langle\left(\psi_{s f, k^{\prime}}^{\dagger}(0)-\psi_{s f, k^{\prime}}(0)\right)\left(d^{\dagger}(0)-d(0)\right)\right\rangle_{0} \\
& +\left\langle\left(\psi_{s f, k}^{\dagger}(\tau)-\psi_{s f, k}(\tau)\right)\left(d^{\dagger}(0)-d(0)\right)\right\rangle_{0}\left\langle\left(d^{\dagger}(\tau)-d(\tau)\right)\left(\psi_{s f, k^{\prime}}^{\dagger}(0)-\psi_{s f, k^{\prime}}(0)\right)\right\rangle_{0} \\
& -\left\langle\left(\psi_{s f, k}^{\dagger}(\tau)-\psi_{s f, k}(\tau)\right)\left(\psi_{s f, k^{\prime}}^{\dagger}(0)-\psi_{s f, k^{\prime}}(0)\right)\right\rangle_{0}\left\langle\left(d^{\dagger}(\tau)-d(\tau)\right)\left(d^{\dagger}(0)-d(0)\right)\right\rangle_{0} .
\end{aligned}
$$

The first term of this autocorrelator is equal to $-\left\langle\hat{I}_{c}(\tau)\right\rangle_{0}\left\langle\hat{I}_{c}(0)\right\rangle_{0}$ and therefore vanishes; after all, there is no net transport in absence of a potential. This term corresponds to the disconnected bubble diagrams that would normally cancel by expanding the partition function, see Appendix A.4. The remaining propagators are all of the form

$$
\begin{aligned}
& \left\langle\left(\alpha^{\dagger}(\tau)-\alpha(\tau)\right)\left(\beta^{\dagger}\left(\tau^{\prime}\right)-\beta\left(\tau^{\prime}\right)\right)\right\rangle_{0} \\
& =\left\langle\alpha^{\dagger}(\tau) \beta^{\dagger}\left(\tau^{\prime}\right)\right\rangle_{0}+\left\langle\alpha(\tau) \beta\left(\tau^{\prime}\right)\right\rangle_{0}-\left\langle\alpha(\tau) \beta^{\dagger}\left(\tau^{\prime}\right)\right\rangle_{0}-\left\langle\alpha^{\dagger}(\tau) \beta\left(\tau^{\prime}\right)\right\rangle_{0} \\
& =-G_{\alpha \beta, 21}\left(\tau-\tau^{\prime}\right)-G_{\alpha \beta, 12}\left(\tau-\tau^{\prime}\right)+G_{\alpha \beta, 11}\left(\tau-\tau^{\prime}\right)+G_{\alpha \beta, 22}\left(\tau-\tau^{\prime}\right) \\
& \equiv \sum_{\mu \nu}^{\prime} G_{\alpha \beta, \mu \nu}\left(\tau-\tau^{\prime}\right)
\end{aligned}
$$

where $\mu, \nu$ denote the components in the Nambu basis, while the prime indicates a signed sum over the components. Plugging this back in, taking into account that the bubble diagrams vanish and writing the Green functions in terms of fermionic Matsubara frequencies $\omega_{n} \equiv \frac{\pi(2 n+1)}{\hbar \beta}$, we obtain

$$
\begin{aligned}
C_{c}^{\tau}(\tau & >0) \\
& =-\frac{e^{2} g_{\perp}^{2}}{4 \hbar^{2} L} \sum_{k, k^{\prime}} \sum_{\mu \nu}^{\prime} \sum_{\rho \sigma}^{\prime}\left(G_{l d, k, \mu \nu}(\tau) G_{l d, k^{\prime}, \rho \sigma}(-\tau)+G_{l l, k k^{\prime}, \mu \nu}(\tau) G_{d d, \rho \sigma}(\tau)\right)
\end{aligned}
$$




$$
\begin{aligned}
= & -\frac{e^{2} g_{\perp}^{2}}{4 \hbar^{2} L} \sum_{k, k^{\prime}} \sum_{\mu \nu}^{\prime} \sum_{\rho \sigma}^{\prime} \frac{1}{(\hbar \beta)_{n, n^{\prime}}^{2}=-\infty} \sum^{\infty}\left(G_{l d, k, \mu \nu}\left(i \omega_{n}\right) G_{l d, k^{\prime}, \rho \sigma}\left(i \omega_{n^{\prime}}\right) e^{-i\left(\omega_{n}-\omega_{n^{\prime}}\right) \tau}\right. \\
& \left.+G_{l l, k k^{\prime}, \mu \nu}\left(i \omega_{n}\right) G_{d d, \rho \sigma}\left(i \omega_{n^{\prime}}\right) e^{-i\left(\omega_{n}+\omega_{n^{\prime}}\right) \tau}\right) .
\end{aligned}
$$

Subsequently transforming the entire expression to bosonic Matsubara frequencies $\Omega_{n} \equiv \frac{2 \pi n}{\hbar \beta}$, we find

$$
\begin{aligned}
C_{c}^{\tau}\left(i \Omega_{n}\right)= & -\frac{e^{2} g_{\perp}^{2}}{4 \hbar^{2} L} \frac{1}{\hbar \beta} \sum_{k, k^{\prime}} \sum_{\mu \nu}^{\prime} \sum_{\rho \sigma}^{\prime} \sum_{n^{\prime}=-\infty}^{\infty}\left(G_{l d, k, \mu \nu}\left(i \omega_{n^{\prime}}\right) G_{l d, k^{\prime}, \rho \sigma}\left(i \omega_{n^{\prime}-n}\right)\right. \\
& \left.+G_{l l, k k^{\prime}, \mu \nu}\left(i \omega_{n^{\prime}}\right) G_{d d, \rho \sigma}\left(-i \omega_{n^{\prime}-n}\right)\right)
\end{aligned}
$$

where it should be noted that $\omega_{n^{\prime}-n}=\omega_{n^{\prime}}-\Omega_{n}$. For analytic continuation to real frequencies, i.e. $C_{c}^{\tau}\left(i \Omega_{n>0}\right) \rightarrow C_{c}^{\mathrm{R}}(\omega)$, the positive frequencies are sufficient, so we will restrict the calculations to $n>0$ from now on. Next, we plug in the Green functions that were listed in Sec. 4.2.3. Working out the matrix multiplications, the participating Green functions are given by

$$
\begin{aligned}
\sum_{\mu \nu}^{\prime} G_{d d, \mu \nu}\left(i \omega_{n}\right)= & 2 D_{b b}\left(i \omega_{n}\right), \\
\sum_{\mu \nu}^{\prime} G_{l d, k, \mu \nu}\left(i \omega_{n}\right)= & \frac{4 g_{\perp}}{\sqrt{L}} \frac{\epsilon_{k}}{\left(\hbar \omega_{n}\right)^{2}+\epsilon_{k}^{2}} D_{b b}\left(i \omega_{n}\right), \\
\sum_{\mu \nu}^{\prime} G_{l l, k k^{\prime}, \mu \nu}\left(i \omega_{n}\right)= & -2 i \hbar \delta_{k, k^{\prime}} \frac{\hbar \omega_{n}}{\left(\hbar \omega_{n}\right)^{2}+\epsilon_{k}^{2}} \\
& +\frac{8 g_{\perp}^{2}}{L} \frac{\epsilon_{k}}{\left(\hbar \omega_{n}\right)^{2}+\epsilon_{k}^{2}} \frac{\epsilon_{k^{\prime}}}{\left(\hbar \omega_{n}\right)^{2}+\epsilon_{k^{\prime}}^{2}} D_{b b}\left(i \omega_{n}\right) .
\end{aligned}
$$

Taking the continuum limit for the sums over $k, k^{\prime}$ and using that all terms that are odd in either $\epsilon_{k}$ or $\epsilon_{k^{\prime}}$ vanish upon integration, the four-point function simplifies to

$$
C_{c}^{\tau}\left(i \Omega_{n>0}\right)=\frac{i e^{2} \Gamma}{2} \frac{1}{\hbar \beta} \sum_{n^{\prime}=-\infty}^{\infty} \int_{-\infty}^{\infty} \frac{\mathrm{d} \epsilon_{k}}{2 \pi \hbar} \frac{\hbar \omega_{n^{\prime}}}{\left(\hbar \omega_{n^{\prime}}\right)^{2}+\epsilon_{k}^{2}} D_{b b}\left(-i \omega_{n^{\prime}-n}\right) .
$$

For future reference, we note that this autocorrelator can be interpreted as a one-loop diagram, with one half of the loop corresponding to a Majorana component of $\mathbf{L}_{0, k}\left(i \omega_{n^{\prime}}\right)$, and the other half to $D_{b b}\left(-i \omega_{n^{\prime}-n}\right)$. 
Let us now consider the remaining sum and integral. Evaluating the integral over $\epsilon_{k} !^{1]}$

$$
\begin{aligned}
C_{c}^{\tau}\left(i \Omega_{n>0}\right) & =\frac{i e^{2} \Gamma}{2 \hbar \beta} \sum_{n^{\prime}=0}^{\infty} \int_{-\infty}^{\infty} \frac{\mathrm{d} \epsilon_{k}}{2 \pi \hbar} \frac{\hbar \omega_{n^{\prime}}}{\left(\hbar \omega_{n^{\prime}}\right)^{2}+\epsilon_{k}^{2}}\left(D_{b b}\left(-i \omega_{n^{\prime}-n}\right)-D_{b b}\left(i \omega_{n^{\prime}+n}\right)\right) \\
& =\frac{i e^{2} \Gamma}{4 \hbar^{2} \beta} \sum_{n^{\prime}=0}^{\infty}\left(D_{b b}\left(-i \omega_{n^{\prime}-n}\right)-D_{b b}\left(i \omega_{n^{\prime}+n}\right)\right)
\end{aligned}
$$

where we used the definition of the fermionic Matsubara frequencies to rewrite the sum over the negative frequencies as a sum over positive ones. In order to make any further progress, it is necessary to find an explicit expression for the $b b$ component of the dot Green function. According to Eqs. (4.41) and (5.10), this component is given by

$$
D_{b b}\left(i \omega_{n}\right)=\frac{\hbar \Gamma}{\pi} \int_{-\infty}^{\infty} \mathrm{d} \epsilon \frac{\epsilon^{2}}{\epsilon^{4}+\left(\Gamma^{2}-2 B^{2}\right) \epsilon^{2}+B^{4}} \frac{1}{i \hbar \omega_{n}-\epsilon} .
$$

We evaluate this integral by returning to contour integration. If $\omega_{n}>0$, we choose a semicircle in the lower half of the imaginary plane to close the contour. Doing so while assuming that $4 B^{2}<\Gamma^{2}$, the contour integral is essentially the same as in Sec. 5.2, with the poles being located at $\epsilon=-i \epsilon_{ \pm}$, see again Eq. (5.18). Meanwhile, if $\omega_{n}<0$, we choose to close the contour in the upper half of the imaginary plane, such that the poles are located at $\epsilon=i \epsilon_{ \pm}$. The corresponding residue picks up an additional minus sign that is cancelled again by taking into account the change in integration direction. Using the knowledge from Sec. 5.2, we therefore find

$$
D_{b b}\left(i \omega_{n}\right)=-\frac{i \hbar}{\Gamma} \frac{1}{\sqrt{1-4\left(\frac{B}{\Gamma}\right)^{2}}}\left(\frac{\epsilon_{+}}{\hbar \omega_{n}+\operatorname{sgn}\left(\omega_{n}\right) \epsilon_{+}}-\frac{\epsilon_{-}}{\hbar \omega_{n}+\operatorname{sgn}\left(\omega_{n}\right) \epsilon_{-}}\right) .
$$

\footnotetext{
${ }^{1}$ This result for the integral assumes that $\omega_{n^{\prime}}$ remains finite, which is not true for all terms of the sum. The actual expression involves $\arctan \left(\Lambda / \hbar \omega_{n^{\prime}}\right)$, where $\Lambda$ is the energy bandwidth (which is usually taken to infinity whenever possible), effectively introducing a cut-off $N$ in the sum over $n^{\prime}$. Although the naive introduction of a hard cut-off $N$ does lead to errors in the expression for the current autocorrelator $C_{c}^{\tau}\left(i \Omega_{n>0}\right)$, the desired dc limit of the linear susceptibility is still exact due to the fact that the erroneous region $\hbar \omega_{n^{\prime}} \sim \Lambda$ does not contribute to the linear order term in $n$. The latter follows from the fact that the autocorrelator only contains the combination $D_{b b}\left(-i \omega_{n^{\prime}-n}\right)-D_{b b}\left(i \omega_{n^{\prime}+n}\right)$ : for terms in the region $\hbar \omega_{n^{\prime}} \sim \Lambda \rightarrow \infty$ (i.e. $n^{\prime} \gg n$ ), this combination is both analytic and even in $n$, see Eq. 6.13). The errors introduced by writing $\arctan \left(\Lambda / \hbar \omega_{n^{\prime}}\right) \rightarrow$ $\operatorname{sgn}\left(\omega_{n^{\prime}}\right) \pi / 2$ therefore only depend on even powers of $n$.
} 
Plugging this back in:

$$
\begin{aligned}
C_{c}^{\tau}\left(i \Omega_{n>0}\right)= & -\frac{e^{2}}{4 \hbar \beta} \frac{1}{\sqrt{1-4\left(\frac{B}{\Gamma}\right)^{2}}} \sum_{\alpha= \pm} \alpha \epsilon_{\alpha} \\
& \times \sum_{n^{\prime}=0}^{\infty}\left(\frac{1}{\hbar \omega_{n^{\prime}-n}+\operatorname{sgn}\left(\omega_{n^{\prime}-n}\right) \epsilon_{\alpha}}+\frac{1}{\hbar \omega_{n^{\prime}+n}+\epsilon_{\alpha}}\right) \\
= & -\frac{e^{2}}{4 \hbar \beta} \frac{1}{\sqrt{1-4\left(\frac{B}{\Gamma}\right)^{2}}} \sum_{\alpha= \pm} \alpha \frac{\beta \epsilon_{\alpha}}{2 \pi} \\
& \times \sum_{n^{\prime}=0}^{\infty}\left(\frac{1}{n^{\prime}-n+\frac{1}{2}+\operatorname{sgn}\left(n^{\prime}-n+\frac{1}{2}\right) \frac{\beta \epsilon_{\alpha}}{2 \pi}}+\frac{1}{n^{\prime}+n+\frac{1}{2}+\frac{\beta \epsilon_{\alpha}}{2 \pi}}\right) \\
= & -\frac{e^{2}}{2 \hbar \beta} \frac{1}{\sqrt{1-4\left(\frac{B}{\Gamma}\right)^{2}}} \sum_{\alpha= \pm} \alpha \frac{\beta \epsilon_{\alpha}}{2 \pi} \\
& \times\left(\sum_{n^{\prime}=0}^{\infty} \frac{1}{n^{\prime}+\frac{1}{2}+\frac{\beta \epsilon_{\alpha}}{2 \pi}}-\sum_{n^{\prime}=0}^{n-1} \frac{1}{n^{\prime}+\frac{1}{2}+\frac{\beta \epsilon_{\alpha}}{2 \pi}}\right) .
\end{aligned}
$$

The first sum of the final equality diverges; it is proportional to $\ln \Lambda$, where $\Lambda$ is the energy bandwidth, see footnote 1 on the previous page. This term is a constant independent of the external Matsubara frequency $\Omega_{n}$, and as a result it does not contribute to the linear susceptibility after performing analytic continuation and applying Eq. 4.56). Working out the second sum (see Appendix A.5 for more details):

$$
C_{c}^{\tau}\left(i \Omega_{n>0}\right)=\text { const. }+\frac{e^{2}}{2 \hbar \beta} \frac{1}{\sqrt{1-4\left(\frac{B}{\Gamma}\right)^{2}}} \sum_{\alpha= \pm} \alpha \frac{\beta \epsilon_{\alpha}}{2 \pi} \Psi\left(\frac{1}{2}+\frac{\beta \epsilon_{\alpha}}{2 \pi}+n\right) .
$$

Finally, we perform the analytic continuation to real frequencies to find

$$
C_{c}^{\mathrm{R}}(\omega)=\text { const. }+\frac{e^{2}}{2 \hbar \beta} \frac{1}{\sqrt{1-4\left(\frac{B}{\Gamma}\right)^{2}}} \sum_{\alpha= \pm} \alpha \frac{\beta \epsilon_{\alpha}}{2 \pi} \Psi\left(\frac{1}{2}+\frac{\beta \epsilon_{\alpha}}{2 \pi}-i \frac{\beta \hbar \omega}{2 \pi}\right) .
$$

Returning to Eq. (4.56) and taking the limit $\omega \rightarrow 0$, we find the dc susceptibility of the charge current:

$$
\chi_{c, \mathrm{dc}}=\frac{e^{2}}{2 h} \frac{1}{\sqrt{1-4\left(\frac{B}{\Gamma}\right)^{2}}}\left(\frac{\beta \epsilon_{+}}{2 \pi} \psi^{(1)}\left(\frac{1}{2}+\frac{\beta \epsilon_{+}}{2 \pi}\right)-\frac{\beta \epsilon_{-}}{2 \pi} \psi^{(1)}\left(\frac{1}{2}+\frac{\beta \epsilon_{-}}{2 \pi}\right)\right) .
$$


Also taking the limits $B^{2} \ll \Gamma^{2}$ (such that we can identify $\epsilon_{+} \approx \Gamma$ as the Kondo energy scale $k_{B} T_{K}$ and $\epsilon_{-} \approx B^{2} / \Gamma$ as the FL crossover energy scale $\left.k_{B} T^{*}\right)$ and $T \ll T_{K}$, we again recover the known charge conductance $G$ of the $\mathrm{C} 2 \mathrm{CK}$ model, but evaluated at $V=0$ :

$$
G=\chi_{c, \mathrm{dc}}=\frac{e^{2}}{2 h}\left(1-\frac{T^{*}}{2 \pi T} \psi^{(1)}\left(\frac{1}{2}+\frac{T^{*}}{2 \pi T}\right)\right) .
$$

The first equality follows from the definition of $G$ from Eq. (5.25) combined with the linear response current $I_{c}=\chi_{c, \mathrm{dc}} V$. Note that Eq. $(6.17)$ is identical to Eq. (5.27) even before taking either of the limits, confirming that the Kubo formula indeed gives the same results as Keldysh formalism.

\subsection{Heat transport}

Following the example of charge transport, we will now consider the significantly more involved case of heat transport. Setting $\mu=0$ for simplicity (i.e. measuring all energies with respect to the equilibrium chemical potential of the system), the heat current operator is equal to the energy current operator from Eq. 4.25). We begin by decomposing the heat current operator into five separate terms: $\hat{I}_{h}=\sum_{i=1}^{5} \hat{I}_{i}$, with

$$
\begin{aligned}
& \hat{I}_{1}=-\frac{\pi v_{F} g_{\perp}}{\sqrt{2} L^{3 / 2}} \sum_{k, k^{\prime}, k^{\prime \prime}}\left(\psi_{c, k^{\prime}}^{\dagger} \psi_{c, k^{\prime \prime}}+\psi_{s, k^{\prime}}^{\dagger} \psi_{s, k^{\prime \prime}}\right)\left(\psi_{s f, k}^{\dagger}-\psi_{s f, k}\right) b, \\
& \hat{I}_{2}=\frac{i \pi v_{F} g_{\perp}}{\sqrt{2} L^{3 / 2}} \sum_{k, k^{\prime}, k^{\prime \prime}} \psi_{f, k^{\prime}}^{\dagger} \psi_{f, k^{\prime \prime}}\left(\psi_{s f, k}^{\dagger}+\psi_{s f, k}\right) a, \\
& \hat{I}_{3}=\frac{i \Lambda g_{\perp}}{2^{3 / 2} \hbar \sqrt{L}} \sum_{k}\left(\psi_{s f, k}^{\dagger}+\psi_{s f, k}\right) a, \\
& \hat{I}_{4}=\frac{\pi v_{F}}{2 L} \sum_{k, k^{\prime}}\left(\epsilon_{k^{\prime}}-\epsilon_{k}\right) \psi_{f, k}^{\dagger} \psi_{f, k^{\prime}} a b, \\
& \hat{I}_{5}=\frac{\pi v_{F}}{2 L} \sum_{k, k^{\prime}}\left(\epsilon_{k^{\prime}}-\epsilon_{k}\right) \psi_{s f, k}^{\dagger} \psi_{s f, k^{\prime}} a b .
\end{aligned}
$$

Here, $a$ and $b$ again refer to the dot Majorana operators, and $\Lambda$ is the energy cut-off that is introduced by writing $\int_{-\infty}^{\infty} \mathrm{d} \epsilon_{k} \rightarrow \int_{-\Lambda}^{\Lambda} \mathrm{d} \epsilon_{k}$. In addition, it is useful to decompose the heat current autocorrelator in a similar way:

$$
C_{h}^{\tau}(\tau>0)=-\sum_{i, j=1}^{5}\left\langle\hat{I}_{i}(\tau) \hat{I}_{j}(0)\right\rangle_{0} \equiv \sum_{i, j=1}^{5} C_{i j}(\tau) .
$$


The main task of this section is thus the identification and subsequent evaluation of all non-zero components of $C_{h}^{\tau}(\tau>0)$, most of which are eight-point functions. The complexity of this task makes it exceedingly difficult to calculate the heat conductance along the FL crossover exactly. Instead, we will restrict ourselves to the NFL fixed point for all calculations involving heat transport, setting the magnetic field to zero ${ }^{2}$

First, we eliminate all components $C_{i j}(\tau)$ that are equal to zero. The first crucial observation is that the $\nu=c, s, f$ modes are all decoupled from the rest of the system in absence of a potential gradient. As is shown in Appendix A.8 the bubble diagrams of the form $\sum_{k, k^{\prime}}\left\langle\psi_{\nu, k}^{\dagger}(\tau) \psi_{\nu, k^{\prime}}(\tau)\right\rangle_{0}$ (i.e. the excitation densities) with $\nu \neq s f$ are therefore all equal to zero. Using Wick's theorem, this already eliminates twelve components, namely $C_{1 i}$ with $i \neq 1, C_{23}, C_{25}$, and their conjugates. Moreover, the flavour modes only contribute to the kinetic energy, such that the fields with different momenta are uncorrelated. As such, the correlator $\left\langle\psi_{f, k}^{\dagger}(\tau) \psi_{f, k^{\prime}}(\tau)\right\rangle_{0}$ is proportional to $\delta_{k, k^{\prime}}$, and the product of this correlator with $\left(\epsilon_{k^{\prime}}-\epsilon_{k}\right)$ is therefore equal to zero as well. This eliminates the components $C_{34}, C_{43}, C_{45}$ and $C_{54}$. Finally, in absence of a magnetic field the combination $\left(C_{35}+C_{53}\right)$ also vanishes as a consequence of the fact that they contain bubble diagrams, but in a non-trivial way. The latter is shown explicitly in Appendix A.8.

As follows from the above considerations, only the diagonal components $C_{i i}$ and the combination $\left(C_{24}+C_{42}\right)$ are non-zero and can potentially contribute to the heat conductance. We will now discuss each of these components separately; supplementary material can be found in Appendix A.9.

\section{- Diagonal component $C_{11}$}

Using that the charge and spin modes are decoupled from the spin-flavour modes and the dot, the first component can be written as

$$
\begin{aligned}
& C_{11}(\tau)=\frac{\left(\pi v_{F} g_{\perp}\right)^{2}}{4 L^{3}} \\
& \times \sum_{\substack{k, k^{\prime}, k^{\prime \prime} \\
q, q^{\prime}, q^{\prime \prime}}}\left\langle\left(\psi_{s f, k}^{\dagger}(\tau)-\psi_{s f, k}(\tau)\right)\left(d^{\dagger}(\tau)-d(\tau)\right)\left(\psi_{s f, q}^{\dagger}(0)-\psi_{s f, q}(0)\right)\left(d^{\dagger}(0)-d(0)\right)\right\rangle_{0} \\
& \times\left\langle\left(\psi_{c, k^{\prime}}^{\dagger}(\tau) \psi_{c, k^{\prime \prime}}(\tau)+\psi_{s, k^{\prime}}^{\dagger}(\tau) \psi_{s, k^{\prime \prime}}(\tau)\right)\left(\psi_{c, q^{\prime}}^{\dagger}(0) \psi_{c, q^{\prime \prime}}(0)+\psi_{s, q^{\prime}}^{\dagger}(0) \psi_{s, q^{\prime \prime}}(0)\right)\right\rangle_{0} .
\end{aligned}
$$

\footnotetext{
${ }^{2}$ We already know that the heat conductance between the leads must be zero in the FL regime, since one of the leads is completely decoupled in that regime. It is therefore only the crossover region that remains challenging with the methods used in this chapter.
} 
To simplify the third line, we refer to the previous observation that the excitation densities corresponding to both the charge modes and the spin modes are equal to zero. The cross terms do therefore not contribute. Meanwhile, the second line is identical to the charge current autocorrelator (up to a constant prefactor) that was already evaluated in the previous section. Eliminating all vanishing terms from the third line and applying the result from Eq. (6.5) to the second line, we find

$$
\begin{aligned}
& C_{11}(\tau) \\
& =-\frac{\left(\pi v_{F} g_{\perp}\right)^{2}}{4 L^{3}} \sum_{\substack{k, k^{\prime}, k^{\prime \prime} \\
q, q^{\prime}, q^{\prime \prime}}} \sum_{\mu \nu}^{\prime} \sum_{\rho \sigma}^{\prime}\left(G_{l d, k, \mu \nu}(\tau) G_{l d, q, \rho \sigma}(-\tau)+G_{l l, k q, \mu \nu}(\tau) G_{d d, \rho \sigma}(\tau)\right) \\
& \quad \times\left(G_{c c, k^{\prime} q^{\prime \prime}, 22}(\tau) G_{c c, k^{\prime \prime} q^{\prime}, 11}(\tau)+G_{s s, k^{\prime} q^{\prime \prime}, 22}(\tau) G_{s s, k^{\prime \prime} q^{\prime}, 11}(\tau)\right) .
\end{aligned}
$$

From Eq. (6.8) it follows that the first term on the right-hand side is odd in both $k$ and $q$, and therefore vanishes upon integrating over these momenta. Transformed to Matsubara frequencies, the above thus becomes

$$
\begin{aligned}
& C_{11}\left(i \Omega_{n}\right)=-\frac{\left(\pi v_{F} g_{\perp}\right)^{2}}{4 L^{3}} \frac{1}{(\hbar \beta)^{3}} \\
& \times \sum_{\substack{k, k^{\prime}, k^{\prime \prime} \\
q, q^{\prime}, q^{\prime \prime}}} \sum_{\mu \nu}^{\prime} \sum_{\rho \sigma} \sum_{n^{\prime}, n^{\prime \prime}, n^{\prime \prime \prime}} G_{l l, k q, \mu \nu}\left(-i\left(\omega_{n^{\prime}}+\omega_{n^{\prime \prime}}+\omega_{n^{\prime \prime \prime}}-\Omega_{n}\right)\right) G_{d d, \rho \sigma}\left(i \omega_{n^{\prime \prime \prime}}\right) \\
& \times\left(G_{c c, k^{\prime} q^{\prime \prime}, 22}\left(i \omega_{n^{\prime}}\right) G_{c c, k^{\prime \prime} q^{\prime}, 11}\left(i \omega_{n^{\prime \prime}}\right)+G_{s s, k^{\prime} q^{\prime \prime}, 22}\left(i \omega_{n^{\prime}}\right) G_{s s, k^{\prime \prime} q^{\prime}, 11}\left(i \omega_{n^{\prime \prime}}\right)\right),
\end{aligned}
$$

where the sums over $n^{\prime}, n^{\prime \prime}$ and $n^{\prime \prime \prime}$ all go from $-\infty$ to $\infty$. Since the charge and spin modes are completely decoupled, the corresponding Green functions satisfy $\mathbf{G}_{c c, k k^{\prime}}\left(i \omega_{n}\right)=\mathbf{G}_{s s, k k^{\prime}}\left(i \omega_{n}\right)=\delta_{k, k^{\prime}} \mathbf{L}_{0, k}\left(i \omega_{n}\right)$, see Eq. 4.40). Plugging in the expressions from Eqs. (6.7) and (6.9), omitting the terms that are odd in any of the momenta and relabelling the remaining momenta:

$$
\begin{aligned}
C_{11}\left(i \Omega_{n}\right)= & \frac{2\left(\pi v_{F} g_{\perp}\right)^{2}}{(L \beta)^{3}} \sum_{k, k^{\prime}, k^{\prime \prime}} \sum_{n^{\prime}, n^{\prime \prime}, n^{\prime \prime \prime}} \frac{1}{i \hbar \omega_{n^{\prime}}-\epsilon_{k}} \frac{1}{i \hbar \omega_{n^{\prime \prime}}-\epsilon_{k^{\prime}}} \\
& \times \frac{1}{i \hbar\left(\omega_{n^{\prime}}+\omega_{n^{\prime \prime}}+\omega_{n^{\prime \prime \prime}}-\Omega_{n}\right)-\epsilon_{k^{\prime \prime}}} D_{b b}\left(i \omega_{n^{\prime \prime \prime}}\right) .
\end{aligned}
$$

Having found an explicit formula for the three-loop diagram $C_{11}\left(i \Omega_{n}\right)$, we continue by evaluating two of the Matsubara sums. Using the Matsubara representation of the Fermi-Dirac distribution from Eq. (5.16), a simple 
partial fraction decomposition leads to the following identity:

$$
\frac{1}{\beta} \sum_{n=-\infty}^{\infty} \frac{1}{i \hbar \omega_{n}-\epsilon} \frac{1}{i \hbar \omega_{n}-\epsilon^{\prime}}=\frac{n_{F}(\epsilon)-n_{F}\left(\epsilon^{\prime}\right)}{\epsilon-\epsilon^{\prime}} .
$$

Furthermore, it is straightforward to show that $n_{F}\left(\epsilon-i \hbar \Omega_{n}\right)=n_{F}(\epsilon)$ and $n_{F}\left(\epsilon-i \hbar \omega_{n}\right)=-n_{B}(\epsilon)$ for bosonic and fermionic Matsubara frequencies, respectively, where $n_{B}(\epsilon)$ is the Bose-Einstein distribution. Applying Eq. 6.29) twice and taking the continuum limit of all momentum sums, we obtain

$$
\begin{aligned}
C_{11}\left(i \Omega_{n}\right)= & \frac{\Gamma}{8 \pi \hbar^{2} \beta} \int_{-\infty}^{\infty} \mathrm{d} \epsilon_{k} \int_{-\infty}^{\infty} \mathrm{d} \epsilon_{k^{\prime}} \int_{-\infty}^{\infty} \mathrm{d} \epsilon_{k^{\prime \prime}} \sum_{n^{\prime}=-\infty}^{\infty} D_{b b}\left(i \omega_{n^{\prime}}\right) \\
& \times \frac{\left(n_{F}\left(\epsilon_{k^{\prime}}\right)-n_{F}\left(\epsilon_{k^{\prime \prime}}\right)\right)\left(n_{F}\left(\epsilon_{k}\right)+n_{B}\left(\epsilon_{k^{\prime \prime}}-\epsilon_{k^{\prime}}\right)\right)}{i \hbar \omega_{n^{\prime}-n}-\left(\epsilon_{k^{\prime \prime}}-\epsilon_{k}-\epsilon_{k^{\prime}}\right)} .
\end{aligned}
$$

Also switching to new variables $\epsilon \equiv\left(\epsilon_{k}+\epsilon_{k^{\prime}}-\epsilon_{k^{\prime \prime}}\right) / 2, \epsilon^{\prime} \equiv\left(\epsilon_{k}-\epsilon_{k^{\prime}}-\epsilon_{k^{\prime \prime}}\right) / 2$, $\epsilon^{\prime \prime} \equiv \epsilon_{k}+\epsilon_{k^{\prime}}+\epsilon_{k^{\prime \prime}}:$

$$
\begin{aligned}
C_{11}\left(i \Omega_{n}\right) & \frac{\Gamma}{8 \pi \hbar^{2} \beta} \int_{-\infty}^{\infty} \mathrm{d} \epsilon \int_{-\infty}^{\infty} \mathrm{d} \epsilon^{\prime} \int_{-\infty}^{\infty} \mathrm{d} \epsilon^{\prime \prime} \sum_{n^{\prime}=-\infty}^{\infty} D_{b b}\left(i \omega_{n^{\prime}}\right) \\
& \times \frac{\left(n_{F}\left(\epsilon-\epsilon^{\prime}\right)-n_{F}\left(-\epsilon+\epsilon^{\prime \prime} / 2\right)\right)\left(n_{F}\left(\epsilon^{\prime}+\epsilon^{\prime \prime} / 2\right)+n_{B}\left(-2 \epsilon+\epsilon^{\prime}+\epsilon^{\prime \prime} / 2\right)\right)}{i \hbar \omega_{n^{\prime}-n}+2 \epsilon} \\
= & \frac{\Gamma}{4 \pi \hbar^{2} \beta} \int_{-\infty}^{\infty} \mathrm{d} \epsilon \int_{-\infty}^{\infty} \mathrm{d} \epsilon^{\prime} \sum_{n^{\prime}=-\infty}^{\infty} \frac{\left(\epsilon+\epsilon^{\prime}\right) \cosh (\beta \epsilon)}{\sinh (\beta \epsilon)+\sinh \left(\beta \epsilon^{\prime}\right)} \frac{1}{i \hbar \omega_{n^{\prime}-n}+2 \epsilon} D_{b b}\left(i \omega_{n^{\prime}}\right) \\
= & \frac{\Gamma}{4 \pi \hbar^{2} \beta} \int_{-\infty}^{\infty} \mathrm{d} \epsilon \sum_{n^{\prime}=-\infty}^{\infty}\left(\frac{\pi^{2}}{2 \beta^{2}}+2 \epsilon^{2}\right) \frac{1}{i \hbar \omega_{n^{\prime}-n}+2 \epsilon} D_{b b}\left(i \omega_{n^{\prime}}\right) \\
\rightarrow & -\frac{\Gamma}{4 \pi \hbar \beta} \int_{-\Lambda^{\prime}}^{\Lambda^{\prime}} \mathrm{d} \epsilon \sum_{n^{\prime}=-\infty}^{\infty}\left(\frac{\pi^{2}}{2 \beta^{2}}+2 \epsilon^{2}\right) \frac{\hbar \omega_{n^{\prime}-n}}{\left(\hbar \omega_{n^{\prime}-n}\right)^{2}+(2 \epsilon)^{2}} \frac{1}{\hbar \omega_{n^{\prime}}+\operatorname{sgn}\left(\omega_{n^{\prime}}\right) \Gamma},
\end{aligned}
$$

where $\Lambda^{\prime}=3 \Lambda / 2$ is the cut-off of the redefined variable $\epsilon$, and we used Eq. 6.13 with $B=0$ for the dot Green function. Next, we write out the 
Matsubara frequencies explicitly, perform the final integral, and take the limit $\Lambda^{\prime} \rightarrow \infty$ (see again footnote 1 of the previous section) to find

$$
\begin{aligned}
C_{11}\left(i \Omega_{n}\right) & =-\frac{\Gamma}{16 \pi \hbar \beta^{2}} \\
& \times \sum_{n^{\prime}=-\infty}^{\infty} \frac{\pi^{2} \operatorname{sgn}\left(n^{\prime}-n+\frac{1}{2}\right)\left(\frac{1}{2}-2\left(n^{\prime}-n+\frac{1}{2}\right)^{2}\right)+4 \beta \Lambda^{\prime}\left(n^{\prime}-n+\frac{1}{2}\right)}{n^{\prime}+\frac{1}{2}+\operatorname{sgn}\left(n^{\prime}+\frac{1}{2}\right) \frac{\beta \Gamma}{2 \pi}} .
\end{aligned}
$$

The most obvious way to calculate the linear susceptibility is to expand the current autocorrelator in $n$ and extract the linear part. However, this is only possible if the correlator is analytic, which the summand of the above expression is not. To work around this, we split the sum into different parts in which the sign functions reduce to constants. Again restricting ourselves to $n>0$, the three different parts are: (i) $n^{\prime}<0$, with both sign functions equal to -1 ; (ii) $0 \leq n^{\prime}<n$, where one of the sign functions is -1 while the other is +1 ; (iii) $n^{\prime} \geq n$, with both sign functions equal to +1 . Writing $n^{\prime} \rightarrow-n^{\prime}-1$ in the first part, using $\sum_{n^{\prime}=n}^{\infty}=\sum_{n^{\prime}=0}^{\infty}-\sum_{n^{\prime}=0}^{n-1}$ in the third part, and subsequently combining the parts that sum over $n^{\prime} \in\{0, \ldots, n-1\}$, we obtain the following analytic form:

$$
\begin{aligned}
C_{11}\left(i \Omega_{n>0}\right)= & -\frac{\Gamma}{16 \pi \hbar \beta^{2}}\left(-2 \pi^{2} \sum_{n^{\prime}=0}^{n-1} \frac{\frac{1}{2}-2\left(n^{\prime}-n+\frac{1}{2}\right)^{2}}{n^{\prime}+\frac{1}{2}+\frac{\beta \Gamma}{2 \pi}}\right. \\
& +\sum_{n^{\prime}=0}^{\infty} \frac{\pi^{2}\left(\frac{1}{2}-2\left(n^{\prime}+n+\frac{1}{2}\right)^{2}\right)+4 \beta \Lambda^{\prime}\left(n^{\prime}+n+\frac{1}{2}\right)}{n^{\prime}+\frac{1}{2}+\frac{\beta \Gamma}{2 \pi}} \\
& \left.+\sum_{n^{\prime}=0}^{\infty} \frac{\pi^{2}\left(\frac{1}{2}-2\left(n^{\prime}-n+\frac{1}{2}\right)^{2}\right)+4 \beta \Lambda^{\prime}\left(n^{\prime}-n+\frac{1}{2}\right)}{n^{\prime}+\frac{1}{2}+\frac{\beta \Gamma}{2 \pi}}\right) .
\end{aligned}
$$

Similar to what we have seen in the case of charge transport, the second and third lines of the above expression diverge, being proportional to $\Lambda^{2}$. However, these lines combined only contain terms that are either constant or quadratic in $n$. For the purpose of finding the linear susceptibility, the above autocorrelator therefore simplifies to

$$
C_{11}\left(i \Omega_{n>0}\right)=\text { const. }+\frac{\pi \Gamma}{8 \hbar \beta^{2}} \sum_{n^{\prime}=0}^{n-1} \frac{\frac{1}{2}-2\left(n^{\prime}-n+\frac{1}{2}\right)^{2}}{n^{\prime}+\frac{1}{2}+\frac{\beta \Gamma}{2 \pi}}+\mathcal{O}\left(\Omega_{n}^{2}\right) .
$$


Finally evaluating the remaining sum, expanding the result to linear order in $n$ (see Appendix A.9), and performing analytic continuation to real frequencies, we find

$$
C_{11}^{\mathrm{R}}(\omega)=\text { const. }-\frac{i \Gamma}{16 \hbar \beta}\left[\frac{\beta \Gamma}{\pi}+\left(\frac{1}{2}-\frac{\beta^{2} \Gamma^{2}}{2 \pi^{2}}\right) \psi^{(1)}\left(\frac{1}{2}+\frac{\beta \Gamma}{2 \pi}\right)\right] \hbar \omega+\mathcal{O}\left(\omega^{2}\right) .
$$

If we furthermore identify $\beta \Gamma$ as $T_{K} / T \rightarrow \infty$ and utilize the expansion of the trigamma function (see Appendix A.5

$$
\frac{1}{x} \psi^{(1)}\left(\frac{1}{2}+\frac{1}{x}\right)=1-\frac{x^{2}}{12}+\mathcal{O}\left(x^{4}\right),
$$

we find that this term of the heat current autocorrelator reduces to

$$
C_{11}^{\mathrm{R}}(\omega)=\text { const. }-\frac{i \pi \omega}{12 \beta^{2}}+\mathcal{O}\left(\omega^{2}\right)
$$

at the NFL fixed point.

\section{- The flavour terms: $C_{22}+C_{44}+C_{24}+C_{42}$}

In order to evaluate these terms, several additional Green functions are required. Referring back to Sec. 4.2 .3 and working out the matrix multiplications, they are given by

$$
\begin{aligned}
\sum_{\mu \nu} G_{d d, \mu \nu}\left(i \omega_{n}\right)= & 2 D_{a a}\left(i \omega_{n}\right)=\frac{2}{i \omega_{n}} \\
\sum_{\mu \nu} G_{l d, k, \mu \nu}\left(i \omega_{n}\right)= & \frac{4 g_{\perp}}{\sqrt{L}} \frac{\hbar \omega_{n}}{\left(\hbar \omega_{n}\right)^{2}+\epsilon_{k}^{2}} D_{b a}\left(i \omega_{n}\right)=0 \\
\sum_{\mu \nu} G_{l l, k k^{\prime}, \mu \nu}\left(i \omega_{n}\right)= & -2 i \hbar \delta_{k, k^{\prime}} \frac{\hbar \omega_{n}}{\left(\hbar \omega_{n}\right)^{2}+\epsilon_{k}^{2}} \\
& -\frac{8 g_{\perp}^{2}}{L} \frac{\hbar \omega_{n}}{\left(\hbar \omega_{n}\right)^{2}+\epsilon_{k}^{2}} \frac{\hbar \omega_{n}}{\left(\hbar \omega_{n}\right)^{2}+\epsilon_{k^{\prime}}^{2}} D_{b b}\left(i \omega_{n}\right),
\end{aligned}
$$

where the unprimed sums denote normal sums over all components. Moreover, we note that

$$
\begin{aligned}
G_{l d, k, 11}\left(i \omega_{n}\right)-G_{l d, k, 22}\left(i \omega_{n}\right)-G_{l d, k, 12}\left(i \omega_{n}\right)+G_{l d, k, 21}\left(i \omega_{n}\right) \\
=\frac{4 i g_{\perp}}{\sqrt{L}} \frac{\hbar \omega_{n}}{\left(\hbar \omega_{n}\right)^{2}+\epsilon_{k}^{2}} D_{b b}\left(i \omega_{n}\right) .
\end{aligned}
$$


In these four equations, we used that the $a$ Majorana fermion is completely free in absence of a magnetic field, and has a zero energy. Going through the same procedure as for $C_{11}$ and using that the sum over all components of $\mathbf{G}_{l d, k}\left(i \omega_{n}\right)$ is equal to zero, we find

$$
\begin{aligned}
& C_{22}\left(i \Omega_{n}\right) \\
& =-\frac{\left(\pi v_{F} g_{\perp}\right)^{2}}{4 L^{3}} \frac{1}{(\hbar \beta)^{3}} \sum_{\substack{k, k^{\prime}, k^{\prime \prime} \\
q, q^{\prime}, q^{\prime \prime}}} \sum_{\mu \nu} \sum_{\rho \sigma} \sum_{n^{\prime}, n^{\prime \prime}, n^{\prime \prime \prime}} G_{f f, k^{\prime} q^{\prime \prime}, 22}\left(i \omega_{n^{\prime}}\right) G_{f f, k^{\prime \prime} q^{\prime}, 11}\left(i \omega_{n^{\prime \prime}}\right) \\
& \quad \times G_{l l, k q, \mu \nu}\left(i \omega_{n^{\prime \prime \prime}}\right) G_{d d, \rho \sigma}\left(-i\left(\omega_{n^{\prime}}+\omega_{n^{\prime \prime}}+\omega_{n^{\prime \prime \prime}}-\Omega_{n}\right)\right) \\
& =\frac{\hbar\left(\pi v_{F} g_{\perp}\right)^{2}}{(L \beta)^{3}} \sum_{k, k^{\prime}, k^{\prime \prime}} \sum_{n^{\prime}, n^{\prime \prime}, n^{\prime \prime \prime}}\left(1+\frac{4 g_{\perp}^{2}}{\hbar L} \sum_{k^{\prime \prime \prime}} \frac{1}{i \hbar \omega_{n^{\prime \prime \prime}}-\epsilon_{k^{\prime \prime \prime}}} D_{b b}\left(i \omega_{n^{\prime \prime \prime}}\right)\right) \\
& \quad \times \frac{1}{i \hbar \omega_{n^{\prime}}-\epsilon_{k}} \frac{1}{i \hbar \omega_{n^{\prime \prime}}-\epsilon_{k^{\prime}}} \frac{1}{i \hbar \omega_{n^{\prime \prime \prime}}-\epsilon_{k^{\prime \prime}}} \frac{1}{i \hbar\left(\omega_{n^{\prime}}+\omega_{n^{\prime \prime}}+\omega_{n^{\prime \prime \prime}}-\Omega_{n}\right)} .
\end{aligned}
$$

Also evaluating the sums in the same way as for $C_{11}$ (i.e. performing two frequency sums using Eq. 6.29), taking the continuum limit of the momentum sums, introducing the coordinates $\epsilon \equiv\left(\epsilon_{k}+\epsilon_{k^{\prime}}\right) / 2, \epsilon^{\prime} \equiv \epsilon_{k}-\epsilon_{k^{\prime}}$, and evaluating the integrals over $\epsilon_{k^{\prime \prime}}, \epsilon_{k^{\prime \prime \prime}}$ and $\left.\epsilon^{\prime}\right)$ :

$$
C_{22}\left(i \Omega_{n}\right)=-\frac{\Gamma}{8 \hbar \beta} \int_{-\Lambda}^{\Lambda} \mathrm{d} \epsilon \sum_{n^{\prime}=-\infty}^{\infty} \frac{\epsilon}{\tanh (\beta \epsilon)} \frac{\hbar \omega_{n^{\prime}-n}}{\left(\hbar \omega_{n^{\prime}-n}\right)^{2}+(2 \epsilon)^{2}} \frac{\hbar \omega_{n^{\prime}}}{\left|\hbar \omega_{n^{\prime}}\right|+\Gamma} .
$$

Before going any further, we also calculate the component

$$
\begin{aligned}
C_{44}(\tau)= & \frac{\left(\pi v_{F}\right)^{2}}{4 L^{2}} \sum_{\substack{k, k^{\prime} \\
q, q^{\prime}}}\left(\epsilon_{k^{\prime}}-\epsilon_{k}\right)\left(\epsilon_{q^{\prime}}-\epsilon_{q}\right)\langle a(\tau) a(0)\rangle_{0}\langle b(\tau) b(0)\rangle_{0} \\
& \times\left\langle\psi_{f, k}^{\dagger}(\tau) \psi_{f, k^{\prime}}(\tau) \psi_{f, q}^{\dagger}(0) \psi_{f, q^{\prime}}(0)\right\rangle_{0} .
\end{aligned}
$$

Once again following the same procedure as for the previous components, this becomes

$$
\begin{aligned}
C_{44}\left(i \Omega_{n}\right)= & \frac{\left(\pi v_{F}\right)^{2}}{4 L^{2} \beta^{3}} \sum_{k, k^{\prime}} \sum_{n^{\prime}, n^{\prime \prime}, n^{\prime \prime \prime}}\left(\epsilon_{k^{\prime}}-\epsilon_{k}\right)^{2} \frac{1}{i \hbar \omega_{n^{\prime}}+\epsilon_{k}} \frac{1}{i \hbar \omega_{n^{\prime \prime}}-\epsilon_{k^{\prime}}} \\
& \times \frac{1}{i \hbar\left(\omega_{n^{\prime}}+\omega_{n^{\prime \prime}}+\omega_{n^{\prime \prime \prime}}-\Omega_{n}\right)} D_{b b}\left(i \omega_{n^{\prime \prime \prime}}\right)
\end{aligned}
$$




$$
=-\frac{1}{2 \hbar \beta} \int_{-\Lambda}^{\Lambda} \mathrm{d} \epsilon \sum_{n^{\prime}=-\infty}^{\infty} \frac{\epsilon^{3}}{\tanh (\beta \epsilon)} \frac{\hbar \omega_{n^{\prime}-n}}{\left(\hbar \omega_{n^{\prime}-n}\right)^{2}+(2 \epsilon)^{2}} \frac{1}{\hbar \omega_{n^{\prime}}+\operatorname{sgn}\left(\omega_{n^{\prime}}\right) \Gamma} .
$$

Finally, without explicitly going through the calculation, the combination $\left(C_{24}+C_{42}\right)$ can analogously be derived to be equal to

$$
C_{24}\left(i \Omega_{n}\right)+C_{42}\left(i \Omega_{n}\right)=-\frac{\Gamma}{\hbar \beta} \int_{-\Lambda}^{\Lambda} \mathrm{d} \epsilon \sum_{n^{\prime}=-\infty}^{\infty} \frac{\epsilon^{3}}{\tanh (\beta \epsilon)} \frac{1}{\left(\hbar \omega_{n^{\prime}-n}\right)^{2}+(2 \epsilon)^{2}} \frac{1}{\left|\hbar \omega_{n^{\prime}}\right|+\Gamma} .
$$

We now extract the contribution of the above four components to the linear susceptibility by combining the components and discussing them together, starting with Eqs. (6.43) and (6.46). Combined, these three terms can be written as

$$
\begin{aligned}
C_{22}\left(i \Omega_{n}\right)+ & C_{24}\left(i \Omega_{n}\right)+C_{42}\left(i \Omega_{n}\right) \\
= & -\frac{\Gamma}{2 \hbar \beta} \int_{-\Lambda}^{\Lambda} \mathrm{d} \epsilon \sum_{n^{\prime}=-\infty}^{\infty} \frac{\epsilon^{3}}{\tanh (\beta \epsilon)} \frac{1}{\left(\hbar \omega_{n^{\prime}-n}\right)^{2}+(2 \epsilon)^{2}} \frac{1}{\left|\hbar \omega_{n^{\prime}}\right|+\Gamma} \\
& -\frac{\Gamma}{8 \hbar \beta} \int_{-\Lambda}^{\Lambda} \mathrm{d} \epsilon \sum_{n^{\prime}=-\infty}^{\infty} \frac{\epsilon}{\tanh (\beta \epsilon)} \frac{1}{\left|\hbar \omega_{n^{\prime}}\right|+\Gamma} \\
& -\frac{\Gamma \Omega_{n}}{8 \beta} \int_{-\Lambda}^{\Lambda} \mathrm{d} \epsilon \sum_{n^{\prime}=-\infty}^{\infty} \frac{\epsilon}{\tanh (\beta \epsilon)} \frac{\hbar \omega_{n^{\prime}-n}}{\left(\hbar \omega_{n^{\prime}-n}\right)^{2}+(2 \epsilon)^{2}} \frac{1}{\left|\hbar \omega_{n^{\prime}}\right|+\Gamma} .
\end{aligned}
$$

The final two lines of this expression do not contribute to the linear susceptibility: the second term does not depend on $n$ at all, while the third term is at least quadratic on $\Omega_{n}$ (to see this, simply note that the summand is odd in $\omega_{n^{\prime}}$ if $\left.n=0\right)$. With that in mind, we unite the four components. Splitting the remaining sums over $n^{\prime}$ into an $n^{\prime}<0$ part and an $n^{\prime} \geq 0$ part, and writing $n^{\prime} \rightarrow-n^{\prime}-1$ in the former, we find

$$
\begin{aligned}
C_{22}\left(i \Omega_{n}\right)+ & C_{44}\left(i \Omega_{n}\right)+C_{24}\left(i \Omega_{n}\right)+C_{42}\left(i \Omega_{n}\right) \\
= & \text { const. }-\frac{1}{2 \hbar \beta} \int_{-\Lambda}^{\Lambda} \mathrm{d} \epsilon \sum_{n^{\prime}=0}^{\infty} \frac{\epsilon^{3}}{\tanh (\beta \epsilon)} \frac{1}{\hbar \omega_{n^{\prime}}+\Gamma} \\
& \times\left(\frac{\hbar \omega_{n^{\prime}+n}+\Gamma}{\left(\hbar \omega_{n^{\prime}+n}\right)^{2}+(2 \epsilon)^{2}}+\frac{\hbar \omega_{n^{\prime}-n}+\Gamma}{\left(\hbar \omega_{n^{\prime}-n}\right)^{2}+(2 \epsilon)^{2}}\right)+\mathcal{O}\left(\Omega_{n}^{2}\right) .
\end{aligned}
$$


Contrary to the previously calculated autocorrelators, the remaining integral cannot be evaluated exactly. As such, we are required to expand in $n$ before having evaluated all of the sums and integrals. Formally, this is the incorrect order of operations, therefore leading to incorrect results if not done carefully. For example, although Eq. (6.48) seems to imply that the remaining sum only contributes to even powers of $n$, this is not necessarily true. The reason for this is hidden in the fact that $\omega_{n^{\prime}-n}<0$ for some of the terms, such that the identities from Appendix A.5 cannot be applied directly. As a result, the sum over the terms involving $\omega_{n^{\prime}-n}$ evaluates to a different analytic function than the sum over the terms that depend on $\omega_{n^{\prime}+n}$. The evaluated sum is thus of the form $(f(-n)+g(n))$ instead of $(f(-n)+f(n))$, therefore generally supplying odd powers of $n$ as well. Taking this into account, we have to explicitly evaluate the sum before expanding it in $n$, see Appendix A.9. Doing so, we find that the resulting power series does indeed contain odd powers of $n$, but the linear term is missing. The combination of components from Eq. 6.48 does therefore not contribute to the linear susceptibility.

\section{- Diagonal component $C_{33}$}

The component $C_{33}$ is very similar to $C_{22}$, such that we can straightforwardly modify the previous steps to find

$$
\begin{aligned}
C_{33}\left(i \Omega_{n}\right) & =-\frac{\left(\Lambda g_{\perp}\right)^{2}}{16 \hbar^{2} L} \frac{1}{\hbar \beta} \sum_{k, k^{\prime}} \sum_{\mu \nu} \sum_{\rho \sigma} \sum_{n^{\prime}=-\infty}^{\infty} G_{l l, k k^{\prime}, \mu \nu}\left(i \omega_{n^{\prime}}\right) G_{d d, \rho \sigma}\left(-i \omega_{n^{\prime}-n}\right) \\
& =-\frac{\Gamma \Lambda^{2}}{16 \hbar \beta} \sum_{n^{\prime}=-\infty}^{\infty} \frac{1}{\hbar \omega_{n^{\prime}-n}} \frac{\hbar \omega_{n^{\prime}}}{\left|\hbar \omega_{n^{\prime}}\right|+\Gamma} .
\end{aligned}
$$

As is shown in Appendix A.9, the latter sum does not contain a linear term in $n$ after evaluation. Consequently, this component does also not contribute to the linear susceptibility.

\section{- Diagonal component $C_{55}$}

This component is by far the most complicated due to the fact that the spin-flavour modes are coupled to the $b$ Majorana mode, combined with the fact that the propagators corresponding to these modes contain non-zero off-diagonal components. Keeping that in mind, Wick's theorem gives us 15 terms to consider. As is discussed in Appendix A.9, five of these terms are vanishing bubble diagrams, while the remaining four bubble diagrams do not have a linear term. For the purpose of finding the linear susceptibility, we therefore only have to consider six terms. Without explicitly performing the lengthy calculation, we note that these combined terms can be expressed 

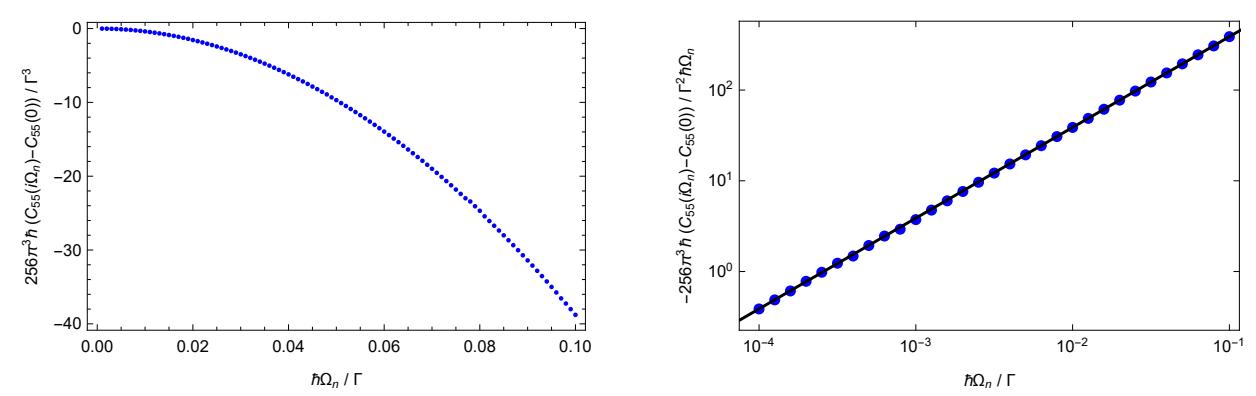

Figure 6.1: The component $C_{55}\left(i \Omega_{n}\right)$ at the NFL fixed point, numerically calculated as a function of dimensionless Matsubara frequency $\hbar \Omega_{n} / \Gamma$ with $\Lambda / \Gamma=10^{2}$. Left: $C_{55}\left(i \Omega_{n}\right)$ minus its zeroth order term, rescaled by a constant prefactor to make it dimensionless. Right: log-log plot of minus the same object, divided by the dimensionless frequency. The solid line is a function of the form $y=a x$ (its slope in the log-log plot therefore being equal to 1), confirming that the susceptibility is perfectly linear in the frequency over this domain. Note that these curves are independent of temperature in the regime $T \ll T_{K}$.

in the following way:

$$
\begin{aligned}
& C_{55}\left(i \Omega_{n}\right) \\
& =\text { const. }+C_{44}\left(i \Omega_{n}\right)-\frac{\left(\pi \hbar v_{F} g_{\perp}\right)^{2}}{(L \hbar \beta)^{3}} \sum_{k, k^{\prime}, k^{\prime \prime}} \sum_{n, n^{\prime}, n^{\prime \prime}}\left(\epsilon_{k}-\epsilon_{k^{\prime}}\right)\left(\epsilon_{k}-\epsilon_{k^{\prime \prime}}\right) \\
& \quad \times \frac{1}{i \hbar \omega_{n^{\prime \prime \prime}}-\epsilon_{k}}\left(\frac{1}{i \hbar \omega_{n^{\prime}}+\epsilon_{k^{\prime}}}-\frac{1}{i \hbar \omega_{n^{\prime \prime}}+\epsilon_{k^{\prime}}}\right) \frac{1}{i \hbar \omega_{n^{\prime \prime}}+\epsilon_{k^{\prime \prime}}} \\
& \quad \times \frac{1}{i \hbar\left(\omega_{n^{\prime}}+\omega_{n^{\prime \prime}}+\omega_{n^{\prime \prime \prime}}-\Omega_{n}\right)} D_{b b}\left(i \omega_{n^{\prime}}\right) D_{b b}\left(i \omega_{n^{\prime \prime}}\right)+\mathcal{O}\left(\Omega_{n}^{2}\right) .
\end{aligned}
$$

In Appendix A.9, we show that the isolated component $C_{44}$ does in fact contain a linear term in $\Omega_{n}$, however, this term goes to zero at the NFL fixed point. As such, $C_{44}$ does not contribute to the linear susceptibility at this point, and we can instead focus on the other terms.

Contrary to all of the previously calculated terms, the remaining terms cannot be calculated exactly, nor can they successfully be expanded in $\Omega_{n}$ before evaluation. The reason for this is the presence of an additional $D_{b b}$ propagator that is interwoven in the sums. Instead of using analytical methods, we therefore calculate the sums numerically as a function of $\Omega_{n}$, and show that the corresponding contribution to the linear susceptibility goes to zero at the NFL fixed point, see Appendix A.9 for the details. The results 

KONDO MODEL

for $\beta \Gamma \rightarrow \infty$ (i.e. at the NFL fixed point) are shown in Fig. 6.1, where we have set the only remaining parameter $\Lambda / \Gamma$ to $10^{2}$ as an example. As can be deduced from the left panel, the lowest non-trivial order term of the component $C_{55}\left(i \Omega_{n}\right)$ is quadratic in $\Omega_{n}$, similar to what we have seen for most of the other components. In addition, the right panel shows a log-log plot of the corresponding contribution to the linear susceptibility $\chi_{55}\left(i \Omega_{n}\right)$ up to a constant prefactor. Upon analytically continuing the data to real frequencies, the plot confirms that this contribution to the susceptibility is perfectly linear in $\omega$ over the entire small- $\omega$ region, such that it goes to zero in the dc limit $\omega \rightarrow 0$.

To summarize, we have shown that only the component $C_{11}$ has a linear term in the frequency at the NFL fixed point. Explicitly, we thus find that the full NFL heat current autocorrelator is given by

$$
C_{h}^{\mathrm{R}}(\omega)=\text { const. }-\frac{i \pi \omega}{12 \beta^{2}}+\mathcal{O}\left(\omega^{2}\right) .
$$

From Eq. 4.56), we now finally obtain the following heat susceptibility:

$$
\chi_{h, \mathrm{dc}}=\frac{\pi^{2} k_{B}^{2} T^{2}}{6 h} .
$$

Returning to the discussion from the final paragraph of Sec. 4.2.5, we briefly consider the off-diagonal terms from Eq. (4.61), which involve correlation functions of the form $\left\langle\hat{I}_{c}(\tau) \hat{I}_{E}(0)\right\rangle_{0}$ and $\left\langle\hat{I}_{E}(\tau) I_{c}(0)\right\rangle_{0}$. Referring back to Eqs. (4.24) and (6.19)-(6.23), we immediately see that any terms involving $\hat{I}_{1}, \hat{I}_{2}$ or $\hat{I}_{4}$ are proportional to vanishing bubble diagrams, see again the first part of this section and Appendix A.8. Moreover, the charge current operator does not contain the $a$ Majorana fermion, such that the products of $\hat{I}_{c}$ with either $\hat{I}_{3}$ or $\hat{I}_{5}$ contain exactly one $a$ operator. At the NFL fixed point, the a Majorana fermion is completely decoupled from all other modes, and all terms involving $\hat{I}_{3}$ and $\hat{I}_{5}$ are therefore equal to zero as well. We thus conclude that the off-diagonal terms from Eq. (4.61) are equal to zero at the NFL fixed point, and as such the temperature gradient does not induce thermopower. Consequently, the two choices $V=0$ and $I_{c}=0$ coincide, such that the heat conductance $\kappa$ is unambiguously given by

$$
\kappa=\frac{\chi_{h, \mathrm{dc}}}{T}=\frac{\pi^{2} k_{B}^{2} T}{6 h}
$$

at the NFL fixed point of the C2CK model. 

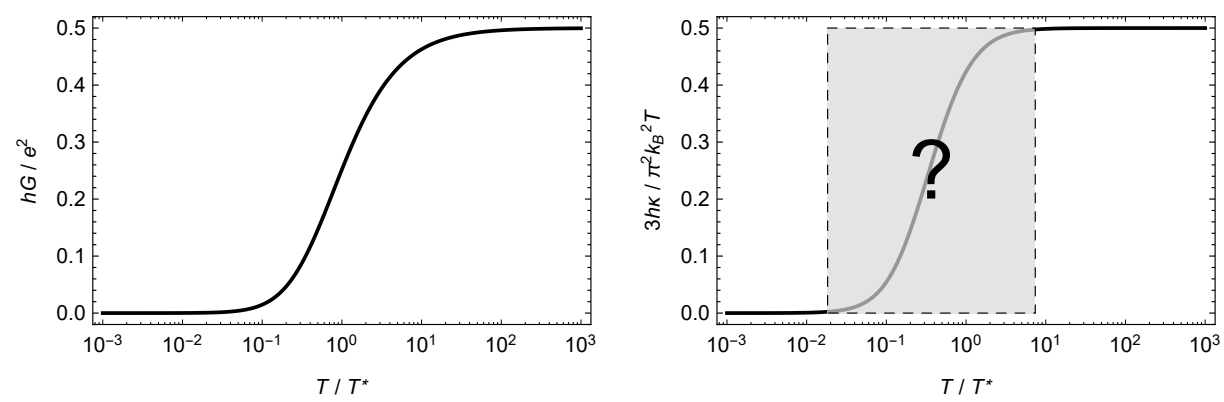

Figure 6.2: Linear response charge conductance $G$ and heat conductance $\kappa$ as functions of dimensionless temperature $T / T^{*}$, both in the limit $T_{K} \rightarrow \infty$. The FL crossover region is not known exactly in the case of the heat conductance.

Finally, this chapter's main results (i.e. the linear response charge and heat conductances from Eqs. (6.18) and (6.53) are plotted in Fig. 6.2, illustrating how the system approaches the NFL fixed point before being driven towards the FL ground state. 



\title{
Chapter 7
}

\section{Wiedemann-Franz law in a non-Fermi liquid and Majorana central charge}

\begin{abstract}
Although previous work on charge transport in the C2CK setup has demonstrated a precise quantitative agreement between theoretical predictions and experimental measurements for the entire universal scaling curve [11, 35. (thereby confirming the theoretical description), there has not yet been any direct experimental evidence of either the NFL or the Majorana character of the system. However, the heat conductance of the NFL fixed point from Eq. (6.53) provides a new angle for studying the C2CK device. Below we will discuss the physical implications of the results from the previous chapters, in particular those concerning the Wiedemann-Franz law at the NFL fixed point and the conformal central charge of the dot.
\end{abstract}

\subsection{Wiedemann-Franz law at the non-Fermi liquid fixed point}

In 1853, Wiedemann and Franz discovered that the electrical and thermal conductivities $\sigma$ and $\kappa$ of conventional metals satisfy a remarkable relation, namely $L \equiv \lim _{T \rightarrow 0} \kappa / T \sigma=L_{0}\left[9\right.$. Here, $L_{0}=\pi^{2} k_{B}^{2} / 3 e^{2}$ is the Lorenz number [10], which is completely independent of the microscopic details and thermodynamic quantities of the setup, and instead only involves fundamental constants. Physically, this relation is caused by the fact that charge and heat are carried by the same itinerant electrons in these metals. The WiedemannFranz law also holds in the context of many nanoelectronic structures [48] (where the conductances play the role of the conductivities); this remains true even in presence of strong electronic correlations, provided that the system can adequately be described by Fermi liquid theory at low temperatures. On the other hand, violations of the Wiedemann-Franz law have been observed in various systems with NFL properties [59 68]. The reason 
for this is that the effective charge and heat carriers in a non-Fermi liquid are not simply bare electrons or even dressed fermionic quasiparticles, but more complicated objects that do not follow FL theory, such that the Wiedemann-Franz law can indeed not be expected to hold. A violation of the Wiedemann-Franz law is therefore often used as an empirical rule of thumb to identify NFL physics.

The C2CK setup provides a rare opportunity to test the WiedemannFranz law at an exactly solvable NFL quantum critical point. Plugging in the exact results from Eqs. (6.18) (with $T^{*}=0$ ) and (6.53), we find

$$
\frac{\kappa}{T G}=\frac{\pi^{2} k_{B}^{2}}{3 e^{2}}
$$

for the NFL fixed point of the C2CK model. The Wiedemann-Franz law is thus satisfied at the NFL fixed point, contrary to the established expectations. On the other hand, the Wiedemann-Franz law is expected to be violated in the FL regime. This can be understood quantitatively by first expanding the conductances in terms of $T / T^{*}$. Doing so, we have

$$
\frac{\kappa}{T G}=\frac{\kappa_{0}+\kappa_{2}\left(T / T^{*}\right)^{2}+\ldots}{T\left(G_{0}+G_{2}\left(T / T^{*}\right)^{2}+\ldots\right)},
$$

where $\kappa_{0,2}$ and $G_{0,2}$ are the first non-zero Taylor coefficients of the conductances 1 Usually, the Wiedemann-Franz law is a statement about the ratio $\kappa_{0} / T G_{0}$. However, in the case of the FL regime both $\kappa_{0}$ and $G_{0}$ are equal to zero (since all transport is blocked at zero temperature), such that $\lim _{T \rightarrow 0} \kappa / T G=\lim _{T \rightarrow 0} \kappa_{2} / T G_{2}$ instead. As such, the Wiedemann-Franz law cannot be expected to hold despite the FL nature of the fixed point. Moreover, from a physical point of view the Lorenz ratio $L$ is expected to be enhanced, which can be understood by realizing that the FL ground state corresponds to a transmission node of the system [69]. Generally, the heat current (and by extension the entropy current) is less sensitive to transmission nodes than the charge current. This is due to the fact that the entropy current is inherently incoherent, while the charge current is not. Coherent currents are more easily blocked, and so the ratio of entropy (or heat) conductance to charge conductance is expected to be enhanced when approaching a transmission node.

It should be noted that both the charge and the heat conductance of the NFL fixed point have an additional factor 1/2 compared to most simple FL

\footnotetext{
${ }^{1}$ The linear coefficient $G_{1}$ can explicitly be shown to vanish by expanding Eq. 6.18 in $T / T^{*}$, while $\kappa_{1}$ is expected to vanish in accordance with Fermi liquid theory.
} 
quantum dot setups, however, the ratio of the two remains unchanged 2 The above therefore demonstrates that it is possible for the Wiedemann-Franz law to be satisfied in a non-Fermi liquid, even though the charge and heat conductances are both individually influenced by the NFL physics. We have thus found a counterexample to the common lore that there is a one-toone relationship between violation of the Wiedemann-Franz law and NFL physics. Although this result is specific to the C2CK setup, the existence of a counterexample proves that violation of the Wiedemann-Franz law cannot be used as a definition of non-Fermi liquids, and that interpreting such a violation as an indication of NFL physics should be done with care.

\subsection{Central charge of the critical system}

The NFL fixed point of the C2CK model is a critical one-dimensional system that can be described by a conformal field theory in $1+1$ dimensions, characterized by the central charge $c$. For such conformal field theories in the unitary limit (i.e. with a transmission coefficient of 1 ), the heat current through a junction is given by

$$
I_{h}=\frac{c \pi^{2}}{6 h} k_{B}^{2}\left(T_{L}^{2}-T_{R}^{2}\right)=\frac{c \pi^{2}}{3 h} k_{B}^{2} T \Delta T+\mathcal{O}\left((\Delta T)^{2}\right),
$$

where $T_{L}=T+\Delta T$ and $T_{R}=T$ are respectively the temperatures of the left and right leads, and $c$ is the central charge of the degrees of freedom involved in the transport processes [70]. This allows us to identify the linear response heat conductance as

$$
\kappa=\frac{c \pi^{2} k_{B}^{2} T}{3 h} .
$$

The C2CK result from Eq. (6.53) thus implies a central charge of $c=1 / 2$, characteristic of the one-dimensional Majorana fermions appearing in the tunnelling term of the Hamiltonian, Eq. (3.22) [29]. Combined with the fact that the heat current is an experimentally observable quantity, this demonstrates that heat transport measurements would provide a direct way

\footnotetext{
${ }^{2}$ At first glance, this shared factor of $1 / 2$ may even seem trivial by interpreting it as a consequence of charge and heat being carried by the same Majorana modes. However, given the complexity of the heat current operator and its dependence on all modes (see Eq. (4.25), this naive interpretation cannot be be used to a priori predict the value of the Lorenz ratio.
} 
to experimentally verify the (usually very elusive) fractionalized Majorana nature of the excitations at the NFL critical point.

We stress that charge transport cannot be used to measure the central charge, despite the fact that the electrical conductance suggestively picks up the same factor of $1 / 2$ as the heat conductance. This is because the electrical conductance generally depends on the geometry of the setup and is therefore not simply proportional to the central charge. For example, the three-channel fixed point of the generalized charge Kondo setup has a charge conductance of $G=2 \sin ^{2}(\pi / 5) e^{2} / h$ [71, which has nothing to do with its central charge. As such, the heat conductance is uniquely suited to experimental observation of the central charge.

\subsection{Comparison with the Goldhaber-Gordon setup}

In addition to studying the critical properties of the C2CK device, we briefly return to the transport properties of Goldhaber-Gordon's spin 2CK setup from Sec. 4.1 and compare the results. As follows from Eqs. 4.8 and 4.9 (setting $\mu=0$ ) together with the values for $t(0)$ as they were discussed in Sec. 4.1, we find that the linear response conductances are given by

$$
G=\frac{\gamma e^{2}}{h}, \quad \kappa=\frac{\gamma \pi^{2} k_{B}^{2} T}{3 h}
$$

at the NFL critical point, where $\gamma$ is again the geometrical factor given by $\gamma=4 \Gamma_{s} \Gamma_{d} /\left(\Gamma_{s}+\Gamma_{d}\right)^{2}$. Moreover, for the one-channel FL ground state in which the source/drain channel remains strongly coupled to the impurity, they become

$$
G=\frac{2 \gamma e^{2}}{h}, \quad \kappa=\frac{2 \gamma \pi^{2} k_{B}^{2} T}{3 h},
$$

while the low-temperature corrections to the FL ground state with a decoupled source/drain channel result in

$$
G=\frac{\pi^{2} e^{2} k_{B}^{2} T^{2}}{3 h} t^{\prime \prime}(0), \quad \kappa=\frac{7 \pi^{4} k_{B}^{4} T^{3}}{15 h} t^{\prime \prime}(0) .
$$

The conductances of the spin $2 \mathrm{CK}$ setup are therefore not universal and depend on the system geometry through $\gamma$. This stands in contrast to the C2CK setup, where the conductances are indeed universal. There is also no interpretation in terms of the central charge, since the Goldhaber-Gordon setup is not in the required unitary limit (the transmission coefficient depends on $\gamma$ instead of being equal to 1). However, the above does still allow 
us to test the Wiedemann-Franz law. In particular, the Wiedemann-Franz law is satisfied at the NFL fixed point as well as in the strongly coupled FL ground state. As such, the critical Goldhaber-Gordon setup provides another example of a non-Fermi liquid that satisfies the Wiedemann-Franz law, further strengthening the conclusions drawn from the $\mathrm{C} 2 \mathrm{CK}$ calculations. On the other hand, the decoupled FL ground state corresponds to a transmission node (similar to the FL ground state of the C2CK setup), and the lowest order corrections lead to $\lim _{T \rightarrow 0} \kappa / T G=7 \pi^{2} k_{B}^{2} / 5 e^{2} \neq L_{0}$. The Lorenz ratio of this particular FL ground state is thus enhanced by a factor $21 / 5$, consistent with our predictions for the C2CK setup.

\subsection{Conclusion}

In this work we theoretically studied charge and heat transport in the C2CK system that was recently experimentally realized [11, complemented by a brief analysis of the spin 2CK setup from Refs. [32 34. We did so by exploiting the exactly solvable effective Emery-Kivelson theory together with RG arguments, paying special attention to the NFL fixed point and the universal FL crossover. Most importantly, we utilized linear response methods to find that the exact expression for the linear heat conductance at the NFL fixed point is given by $\kappa=\pi^{2} k_{B}^{2} T / 6 h$. This novel result was used to show that the Wiedemann-Franz law is satisfied at this point, crucially providing a counterexample to the common lore that violation of the Wiedemann-Franz law is a defining feature of non-Fermi liquids. Furthermore, we showed that heat transport provides direct access to the central charge $c=1 / 2$ of the critical theory, thereby exposing the elusive Majorana nature of the effective degrees of freedom that mediate transport through the dot. Given the high tunability of the setup from Ref. [11] and its capability to probe the NFL critical point, these results are within reach of existing experiments. 



\section{Chapter 8}

\section{Extensions}

In this final chapter, we will discuss several extensions of the research presented in this thesis, such as possible methods for calculating the heat conductance along the FL crossover and perturbations away from the EK point. Afterwards, we will conclude with a brief outlook on future research directions concerning multichannel Kondo models, both on a theoretical and an experimental level.

\subsection{Heat transport away from the non-Fermi liquid fixed point}

So far, we have only considered heat transport at the NFL fixed point and in the FL ground state, arguing that it is much more difficult to calculate the heat conductance along the FL crossover. Moreover, the methods from Chapter 6 are ill-suited to numerical evaluation of the required linear susceptibilities. The reason for the latter is that Matsubara techniques inherently involve analytic continuation to real frequencies, while some of the required Green functions are not analytic. As we have seen in Chapter 6 and Appendix A.9, working around this problem demands significant analytical effort before numerical methods can be applied, and even then the calculations are restricted to the limit $\beta \Gamma \rightarrow \infty$. To complement our results, we will therefore propose two alternative strategies for performing linear response heat transport calculations away from the NFL fixed point.

The first strategy is to calculate the current autocorrelator from Eq. 4.57) directly, without falling back to Matsubara techniques. This can be done by using the Keldysh techniques from Chapter 5. Although this may seem to contradict the previous arguments for Keldysh techniques not being suitable for heat transport calculations, the usual problems are avoided by combining Keldysh formalism with linear response theory. In particular, and contrary to Chapter 5, the goal is now to calculate an equilibrium expectation value 
in absence of any bias between the leads, which is well within reach of the more general non-equilibrium Keldysh formalism. The actual calculation is nevertheless exceedingly complicated, even more so than those from Sec. 6.2 due to the added Keldysh matrix structure and the general complexity of finding the Keldysh component of a propagator. However, the calculation ultimately only involves integration without the subtleties and complications of analytic continuation, making this method much more suitable for a numerical study of heat transport along the FL crossover.

Alternatively, the low-temperature corrections to the heat conductance of the FL ground state can be studied with perturbation theory, making it possible to calculate the corresponding Lorenz ratio. To do so, one first has to recall that the FL ground state is equivalent to a one-channel setup in which one of the leads (say the right lead) is completely decoupled from the rest of the system, i.e. $J_{\lambda}^{R R} / J_{\perp}^{L L} \rightarrow 0$. The idea is to treat $J_{\lambda}^{R R}$ as a small perturbation to the one-channel setup, while keeping $J_{\lambda}^{L R}=0$. Similar to the $2 \mathrm{CK}$ model, the one-channel Kondo model has an exactly solvable point corresponding to a specific value of $J_{z}^{L L}$ [44, 51]. At this original Toulouse point, the unperturbed system (i.e. with $J_{\lambda}^{R R}=0$ ) is described by the following non-interacting effective Hamiltonian:

$$
\hat{H}_{0}=\sum_{\nu=c, s} \sum_{k} \epsilon_{k} \psi_{\nu, k}^{\dagger} \psi_{\nu, k}+\sum_{k, \sigma} \epsilon_{k} \psi_{R k \sigma}^{\dagger} \psi_{R k \sigma}+\frac{J_{\perp}^{L L}}{2 \sqrt{2 \pi a_{0}}}\left(\psi_{s}^{\dagger}(0) d+d^{\dagger} \psi_{s}(0)\right),
$$

where $d \equiv \tau^{-}$represents the dot, and $\psi_{\nu, k}$ are composite fields consisting of all modes $\psi_{L k \sigma}$ living on the left lead (similar to the $\nu=c, s, f, s f$ fields from the Emery-Kivelson mapping procedure). With respect to this mapped Hamiltonian, the coupling between the right lead to the dot enters through the interaction terms

$$
\begin{aligned}
\hat{H}_{I}= & \frac{J_{\perp}^{R R}}{2}\left(\psi_{R \uparrow}^{\dagger}(0) \psi_{R \downarrow}(0) d+\psi_{R \downarrow}^{\dagger}(0) \psi_{R \uparrow}(0) d^{\dagger}\right) \\
& +\frac{J_{z}^{R R}}{2}\left(\psi_{R \uparrow}^{\dagger}(0) \psi_{R \uparrow}(0)-\psi_{R \downarrow}^{\dagger}(0) \psi_{R \downarrow}(0)\right)\left(d^{\dagger} d-\frac{1}{2}\right) \\
= & \frac{J_{\perp}^{R R}}{2}\left[s_{R R}^{x}\left(d^{\dagger}+d\right)-i s_{R R}^{y}\left(d^{\dagger}-d\right)\right]+J_{z}^{R R} s_{R R}^{z}\left(d^{\dagger} d-\frac{1}{2}\right) .
\end{aligned}
$$

Note that the one-channel Toulouse point Hamiltonian $\hat{H}_{0}$ does not contain any Majorana operators. However, the addition of the right lead to the onechannel setup naturally involves the Majorana combinations $\left(d^{\dagger}+d\right)$ and $\left(d^{\dagger}-d\right)$, demonstrating how Majorana modes can emerge in two-channel 
models. With the above in mind, the corrections to the heat conductance of the FL ground state can now be found by deriving the appropriate current operator from Eqs. (8.1) and 8.2), then calculating the required expectation value using perturbation theory in $J_{\lambda}^{R R}$. The resulting perturbative expression for the linear susceptibility in terms of bare Green functions can subsequently be evaluated using either Matsubara or Keldysh techniques. While the final integrals are still rather complicated, this strategy reduces the complexity of the problem to the same level as that of the heat transport calculations from Sec. 6.2, thus potentially allowing for analytical results.

\subsection{Beyond the universal Fermi liquid crossover}

As was mentioned in Sec. 3.6, perturbation theory can be used to find the corrections to the NFL results in terms of a finite $T / T_{K}$. In this section, we will explicitly calculate the corrections to the linear response charge conductance away from the EK point to lowest order in $\lambda \equiv 2 \pi \hbar v_{F}-J_{z}$ and $T / T_{K}$. Our starting point is the interaction term from Eq. (3.16),

$$
\begin{aligned}
\hat{H}_{I} & =\lambda: \psi_{s}^{\dagger}(0) \psi_{s}(0):\left(d^{\dagger} d-\frac{1}{2}\right) \\
& =\frac{i \lambda}{L} b a \sum_{k, k^{\prime}}: \psi_{s, k}^{\dagger} \psi_{s, k^{\prime}}:
\end{aligned}
$$

which we will treat as a perturbation to the non-interacting Hamiltonian from Eq. (3.22) [45]. In order to understand how the linear susceptibility changes in presence of this interaction term, we will have to find the corrections to Eq. (6.10), which should be understood as

$$
C_{c}^{\tau}\left(i \Omega_{n>0}\right)=-\frac{e^{2} \Gamma}{8 \pi \hbar^{3} \beta} \sum_{n^{\prime}=-\infty}^{\infty} \int_{-\infty}^{\infty} \mathrm{d} \epsilon_{k} \operatorname{Tr}\left[\mathbf{L}_{0, k}\left(i \omega_{n^{\prime}}\right)\right] D_{b b}\left(-i \omega_{n^{\prime}-n}\right) .
$$

Since the interaction term does not involve spin-flavour modes, the bare propagators corresponding to those modes remain unchanged. Our first objective is thus to find the corrections to the $b b$ component of the dot Green function in presence of a non-zero $\lambda$. While doing so, we will set the magnetic field $B$ to zero (i.e. $T^{*}=0$ ), restricting the analysis purely to the NFL regime and removing any effects of the FL regime in the process.

We approximate the full $b b$ component of the dot Green function $D_{b b}^{\text {full }}\left(i \omega_{n}\right)$ in presence of interactions by employing standard Feynman techniques. Using that the interaction Hamiltonian from Eq. 8.3 provides a four-point 
Table 8.1: Definitions of the different components of the Feynman diagrams. The arrow in the fourth diagram indicates the propagation direction of $\psi_{s, k}$.

\begin{tabular}{|c|c||c|}
\hline Expression & Diagram & Vertex \\
\hline \hline$D_{b b}^{\text {full }}\left(i \omega_{n}\right)$ & $\underline{\underline{\omega_{n}}}$ \\
\hline$D_{b b}\left(i \omega_{n}\right)$ & $\overline{\omega_{n}}$ \\
\hline$D_{a a}\left(i \omega_{n}\right)$ & $\sim \omega_{n}$ \\
\hline$\frac{1}{L} \sum_{k} G_{s, k}\left(i \omega_{n}\right)$ & $-\overrightarrow{\omega_{n}}$ & \\
\hline
\end{tabular}

vertex involving two $\psi_{s, k}$ legs, an $a$ leg and a $b$ leg, the Feynman rules lead to the following diagrammatic expression for $D_{b b}^{\text {full }}\left(i \omega_{n}\right)$ :

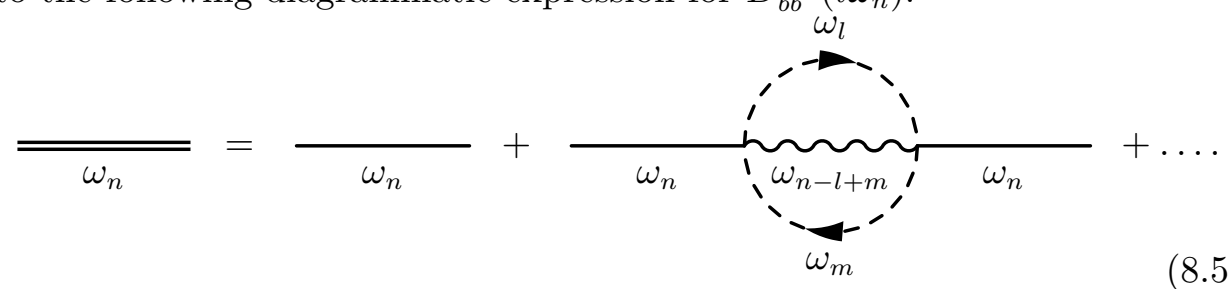

Here, each vertex comes with a prefactor $i \lambda / \hbar^{2} \beta$ and a sum over Matsubara frequencies; the definitions of the other components can be found in Table 8.1. Reading off the above Feynman diagrams, we find that the lowest order of the self-energy is given by

$\Sigma\left(i \omega_{n}\right)=-\frac{\lambda^{2}}{\hbar^{2} L^{2}} \frac{1}{(\hbar \beta)^{2}} \sum_{n^{\prime}, n^{\prime \prime}} \sum_{k, k^{\prime}} D_{a a}\left(-i\left(\omega_{n^{\prime}}-\omega_{n^{\prime \prime}}-\omega_{n}\right)\right) G_{s, k}\left(i \omega_{n^{\prime}}\right) G_{s, k^{\prime}}\left(i \omega_{n^{\prime \prime}}\right)$,

where $G_{s, k}\left(i \omega_{n}\right)$ is shorthand notation for $G_{s s, k k, 11}\left(i \omega_{n}\right)$. A more detailed and explicit derivation of this expression can be found in Appendix A.10.

Using that the $a$ and $\psi_{s, k}$ modes are completely isolated from the rest of the system if $B=0$ and $\lambda=0$, and taking the continuum limit of the sums over $k$ and $k^{\prime}$, we have

$$
\begin{aligned}
\Sigma\left(i \omega_{n}\right)= & -\frac{\lambda^{2}}{\hbar v_{F}^{2}} \frac{1}{\beta^{2}} \int_{-\infty}^{\infty} \frac{\mathrm{d} \epsilon_{k}}{2 \pi \hbar} \int_{-\infty}^{\infty} \frac{\mathrm{d} \epsilon_{k^{\prime}}}{2 \pi \hbar} \sum_{n^{\prime}, n^{\prime \prime}} \frac{1}{i \hbar\left(\omega_{n}-\omega_{n^{\prime}}+\omega_{n^{\prime \prime}}\right)} \\
& \times \frac{1}{i \hbar \omega_{n^{\prime}}-\epsilon_{k}} \frac{1}{i \hbar \omega_{n^{\prime \prime}}-\epsilon_{k^{\prime}}} .
\end{aligned}
$$


Furthermore applying Eq. (6.29) twice, together with the substitutions $\epsilon \equiv$ $\left(\epsilon_{k}+\epsilon_{k^{\prime}}\right) / 2, \epsilon^{\prime} \equiv \epsilon_{k}-\epsilon_{k^{\prime}}$, the self-energy becomes

$$
\begin{aligned}
\Sigma\left(i \omega_{n}\right) & =\frac{\lambda^{2}}{\hbar v_{F}^{2}} \int_{-\infty}^{\infty} \frac{\mathrm{d} \epsilon_{k}}{2 \pi \hbar} \int_{-\infty}^{\infty} \frac{\mathrm{d} \epsilon_{k^{\prime}}}{2 \pi \hbar} \frac{\left(n_{F}(0)-n_{F}\left(\epsilon_{k^{\prime}}\right)\right)\left(n_{F}\left(\epsilon_{k}\right)+n_{B}\left(\epsilon_{k^{\prime}}\right)\right)}{i \hbar \omega_{n}-\left(\epsilon_{k}-\epsilon_{k^{\prime}}\right)} \\
& =\frac{\lambda^{2}}{4 \hbar v_{F}^{2}} \int_{-\infty}^{\infty} \frac{\mathrm{d} \epsilon_{k}}{2 \pi \hbar} \int_{-\infty}^{\infty} \frac{\mathrm{d} \epsilon_{k^{\prime}}}{2 \pi \hbar} \frac{\cosh \left(\beta\left(\epsilon_{k}+\epsilon_{k^{\prime}}\right) / 2\right)}{\cosh \left(\beta \epsilon_{k} / 2\right) \cosh \left(\beta \epsilon_{k^{\prime}} / 2\right)} \frac{1}{i \hbar \omega_{n}-\left(\epsilon_{k}+\epsilon_{k^{\prime}}\right)} \\
& =\frac{\lambda^{2}}{2 \hbar v_{F}^{2}} \int_{-\infty}^{\infty} \frac{\mathrm{d} \epsilon}{2 \pi \hbar} \int_{-\infty}^{\infty} \frac{\mathrm{d} \epsilon^{\prime}}{2 \pi \hbar} \frac{\cosh (\beta \epsilon)}{\cosh (\beta \epsilon)+\cosh \left(\beta \epsilon^{\prime} / 2\right)} \frac{1}{i \hbar \omega_{n}-2 \epsilon} \\
& =\frac{\lambda^{2}}{\pi \hbar^{2} v_{F}^{2}} \int_{-\infty}^{\infty} \frac{\mathrm{d} \epsilon}{2 \pi \hbar} \frac{\epsilon}{\tanh (\beta \epsilon)\left(i \hbar \omega_{n}-2 \epsilon\right)}
\end{aligned}
$$

where we wrote $\epsilon_{k^{\prime}} \rightarrow-\epsilon_{k^{\prime}}$ in the second line. In order to deal with the remaining UV divergence, we reintroduce the energy cut-off $\Lambda$. Noting that the real part of the integrand is odd in $\epsilon$, we obtain

$$
\Sigma\left(i \omega_{n}\right)=-\frac{i \omega_{n} \lambda^{2}}{2 \pi^{2} \hbar^{2} v_{F}^{2}} \int_{-\Lambda}^{\Lambda} \mathrm{d} \epsilon \frac{\epsilon}{\tanh (\beta \epsilon)} \frac{1}{\left(\hbar \omega_{n}\right)^{2}+(2 \epsilon)^{2}},
$$

which diverges logarithmically as $\Lambda \rightarrow \infty$.

We now return to the autocorrelator from Eq. (8.4), replacing $D_{b b}\left(i \omega_{n}\right)$ with $D_{b b}^{\text {full }}\left(i \omega_{n}\right)$ and evaluating the momentum integral. The methods for dealing with momentum integrals remain unchanged with respect to Sec.6.1. such that the correlation function becomes

$$
\begin{aligned}
C_{c}^{\tau}\left(i \Omega_{n>0}\right) \\
=\frac{i e^{2} \Gamma}{4 \hbar^{2} \beta} \sum_{n^{\prime}=0}^{\infty}\left(D_{b b}^{\text {full }}\left(-i \omega_{n^{\prime}-n}\right)-D_{b b}^{\text {full }}\left(i \omega_{n^{\prime}+n}\right)\right) \\
=-\frac{i e^{2} \Gamma}{4 \hbar^{2} \beta} \sum_{n^{\prime}=0}^{\infty}\left(\left(D_{b b}\left(i \omega_{n^{\prime}-n}\right)\right)^{2} \Sigma\left(i \omega_{n^{\prime}-n}\right)+\left(D_{b b}\left(i \omega_{n^{\prime}+n}\right)\right)^{2} \Sigma\left(i \omega_{n^{\prime}+n}\right)\right) \\
\\
\quad+\left.C_{c}^{\tau}\left(i \Omega_{n>0}\right)\right|_{\lambda=0}+\mathcal{O}\left(\lambda^{4}\right),
\end{aligned}
$$

where we used the fact that both $D_{b b}\left(i \omega_{n}\right)$ and $\Sigma\left(i \omega_{n}\right)$ are odd functions of $\omega_{n}$. Reading off $D_{b b}\left(i \omega_{n}\right)$ from Eq. 6.13) (taking the limit $B \rightarrow 0$ ) and 
splitting the sum in the same way as in Eq. (6.14), the lowest order correction to the current autocorrelator can be written as

$$
\begin{aligned}
\Delta C_{c}^{\tau}\left(i \Omega_{n>0}\right) & \\
= & \frac{i e^{2} \Gamma}{2 \beta}\left(\sum_{n^{\prime}=0}^{\infty} \frac{\Sigma\left(i \omega_{n^{\prime}}\right)}{\left(\hbar \omega_{n^{\prime}}+\Gamma\right)^{2}}-\sum_{n^{\prime}=0}^{n-1} \frac{\Sigma\left(i \omega_{n^{\prime}}\right)}{\left(\hbar \omega_{n^{\prime}}+\Gamma\right)^{2}}\right) \\
& =\text { const. }-\frac{e^{2} \Gamma \lambda^{2}}{4 \pi^{2} \hbar^{3} v_{F}^{2} \beta} \int_{-\Lambda}^{\Lambda} \mathrm{d} \epsilon \sum_{n^{\prime}=0}^{n-1} \frac{\epsilon}{\tanh (\beta \epsilon)} \frac{1}{\left(\hbar \omega_{n^{\prime}}+\Gamma\right)^{2}} \frac{\hbar \omega_{n^{\prime}}}{\left(\hbar \omega_{n^{\prime}}\right)^{2}+(2 \epsilon)^{2}} .
\end{aligned}
$$

The sum over the Matsubara frequencies can be evaluated by using the partial fraction decomposition

$$
\begin{aligned}
\frac{1}{\left(\hbar \omega_{n^{\prime}}+\Gamma\right)^{2}} \frac{\hbar \omega_{n^{\prime}}}{\left(\hbar \omega_{n^{\prime}}\right)^{2}+(2 \epsilon)^{2}} & =\frac{1}{\left(\hbar \omega_{n^{\prime}}+\Gamma\right)^{2}} \frac{\Gamma}{\Gamma^{2}+(2 \epsilon)^{2}}-\frac{1}{\hbar \omega_{n^{\prime}}+\Gamma} \frac{\Gamma^{2}-(2 \epsilon)^{2}}{\left(\Gamma^{2}+(2 \epsilon)^{2}\right)^{2}} \\
& +\frac{1}{\hbar \omega_{n^{\prime}}-2 i \epsilon} \frac{1}{2(\Gamma+2 i \epsilon)^{2}}+\frac{1}{\hbar \omega_{n^{\prime}}+2 i \epsilon} \frac{1}{2(\Gamma-2 i \epsilon)^{2}}
\end{aligned}
$$

and applying the digamma function identities from Appendix A.5. Subsequently expanding the result to linear order in $\Omega_{n}$ and to lowest order in $1 / \beta \Gamma$, we find

$$
\begin{aligned}
& \Delta C_{c}^{\tau}\left(i \Omega_{n>0}\right) \\
& =\text { const. }-\frac{e^{2} \beta \Gamma \lambda^{2}}{32 \pi^{4} \hbar^{3} v_{F}^{2}} \int_{-\beta \Lambda}^{\beta \Lambda} \mathrm{d}(\beta \epsilon) \frac{\beta \epsilon}{\tanh (\beta \epsilon)} \\
& \quad \times\left[\left(\psi^{(1)}\left(\frac{1}{2}-\frac{i \beta \epsilon}{\pi}\right)+\psi^{(1)}\left(\frac{1}{2}+\frac{i \beta \epsilon}{\pi}\right)\right) \frac{\hbar \Omega_{n}}{(\beta \Gamma)^{2}}+\mathcal{O}\left(\Omega_{n}^{2},(1 / \beta \Gamma)^{3}\right)\right] .
\end{aligned}
$$

Finally evaluating the remaining integral in the wide-band limit $\Lambda \rightarrow \infty$ and performing analytic continuation to real frequencies, we find the explicit lowest order correction to the dc linear susceptibility:

$$
\Delta \chi_{c, \mathrm{dc}}=-\frac{\pi^{3} e^{2} \lambda^{2}}{16 h^{3} v_{F}^{2}} \frac{1}{\beta \Gamma}+\mathcal{O}\left((1 / \beta \Gamma)^{2}\right) .
$$


Identifying $1 / \beta \Gamma$ as $T / T_{K}$, the charge conductance is therefore equal to

$$
G=\frac{e^{2}}{2 h}\left(1-\frac{\pi^{3} \lambda^{2}}{8 h^{2} v_{F}^{2}} \frac{T}{T_{K}}+\ldots\right)
$$

when approaching the local moment fixed point from below, where the dots contain all higher order terms in products of $\lambda^{2}$ and $T / T_{K}$. This agrees with the results from previous research $[72-74$. Note that the full linear term in $T / T_{K}$ is by itself a series in $\lambda^{2}$, while $\lambda$ is not necessarily small.

A few remarks are in order. (i) The linear order correction (in $T / T_{K}$ ) is found to remain finite as $\Lambda \rightarrow \infty$. However, if the cut-off is taken all the way to infinity, the higher order terms take over, since they are proportional to $\ln \Lambda$. As a result, the lowest order correction to the dc conductance is only linear in $T / T_{K}$ if the cut-off is finite. This is always the case in real systems. (ii) The leading order correction to the EK point NFL conductance is quadratic in $T / T_{K}$, as can be read off from Eq. (6.17) by using Eq. (6.36). This stands in contrast to the temperature dependence away from the EK point, which we found to be linear. We thus find that the lowest order correction to the NFL conductance is linear in $T / T_{K}$ at a general point in parameter space (including the $\mathrm{C} 2 \mathrm{CK}$ model), but that the linear term vanishes at the fundamentally different EK point (i.e. $\lambda=0$ ), where the lowest order correction is quadratic. This linear behaviour of the C2CK NFL conductance agrees with the known experimental results [11, 35]. (iii) The lowest order correction to the conductance vanishes as $T / T_{K}$ goes to zero, independent of $\lambda$. This is a manifestation of the irrelevance of the anisotropy $\Delta J_{z} \equiv J_{z}-J_{\perp}$ : no matter the starting point (which is dictated by the parameter $\lambda$ ), the RG flow ensures that $\Delta J_{z}$ effectively goes to zero with the energy scale (in this case $T / T_{K}$ ), such that the EK point results become exact regardless of $\lambda$. (iv) Looking back at the methods used in this section, it becomes clear that analytically calculating the corrections to the heat conductance remains challenging: the lowest order self-energy is a twoloop diagram, so finding the corrections to the heat conductance (which by itself involves many three-loop diagrams) would require calculating five-loop diagrams. That is a task well beyond the scope of this work.

\subsection{The one-dimensional two-lead Kondo lattice}

Finally, we consider a one-dimensional lattice extension of the 2CK model from Sec. 3.1, providing a look into the field of Kondo lattices while illustrating how a simple extension can pave the way towards novel multichannel 


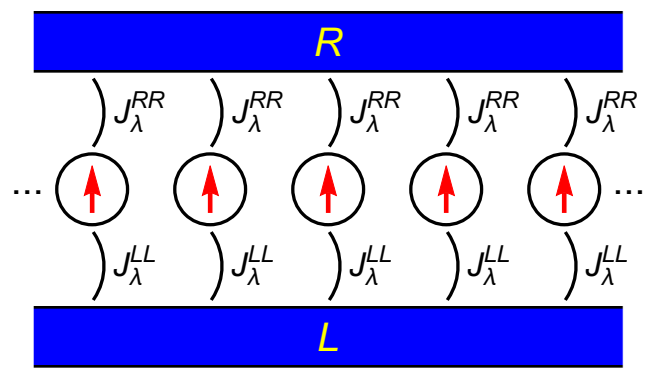

Figure 8.1: Schematic of the one-dimensional lattice extension of the 2CK model. Note that impurity-mediated cotunnelling between the leads is not included in the present discussion, such that $J_{\lambda}^{L R}$ is absent.

Kondo physics. Specifically, we construct a two-lead Kondo lattice by taking two identical metallic leads, which we connect to a chain of independent Kondo impurities at equal spacing, see Fig. 8.1. The introduction of a onedimensional lattice structure breaks the rotational symmetry of the original 2CK model; as a result, the leads can no longer be described by effective left-movers only. Instead, we enforce a one-dimensional nature on the leads by modelling them as chains as well, such that the leads host both leftand right-moving electrons. Furthermore assuming a symmetric configuration (i.e. setting $J_{\lambda}^{L L}=J_{\lambda}^{R R} \equiv J_{\lambda}$ ), and in absence of $J_{\lambda}^{L R}$ and $B$, the Hamiltonian of the lattice model is given by

$$
\begin{aligned}
\hat{H}= & i \hbar v_{F} \sum_{\alpha} \sum_{\sigma} \int_{-\infty}^{\infty} \mathrm{d} x\left[\psi_{l \alpha \sigma}^{\dagger}(x) \partial_{x} \psi_{l \alpha \sigma}(x)-\psi_{r \alpha \sigma}^{\dagger}(x) \partial_{x} \psi_{r \alpha \sigma}(x)\right] \\
& +\sum_{j, \eta, \alpha}\left[\frac{J_{\perp}}{2}\left(\psi_{\eta \alpha \uparrow}^{\dagger}\left(x_{j}\right) \psi_{\eta \alpha \downarrow}\left(x_{j}\right) \tau_{j}^{-}+\psi_{\eta \alpha \downarrow}^{\dagger}\left(x_{j}\right) \psi_{\eta \alpha \uparrow}\left(x_{j}\right) \tau_{j}^{+}\right)\right. \\
& \left.+\frac{J_{z}}{2}\left(\psi_{\eta \alpha \uparrow}^{\dagger}\left(x_{j}\right) \psi_{\eta \alpha \uparrow}\left(x_{j}\right)-\psi_{\eta \alpha \downarrow}^{\dagger}\left(x_{j}\right) \psi_{\eta \alpha \downarrow}\left(x_{j}\right)\right) \tau_{j}^{z}\right],
\end{aligned}
$$

see also Sec. 3.1. Here, the operators $\psi_{\eta \alpha \sigma}(x)$ with $\eta=l, r$ respectively correspond to the left- and right-moving electrons on lead $\alpha=L, R$ with spin $\sigma=\uparrow, \downarrow$. These electrons are coupled to a one-dimensional lattice of localized spin states $\boldsymbol{\tau}_{j}$ (acting as the Kondo impurities, located at the positions $x_{j}$ ) through the anisotropic coupling constant $J_{\lambda}$, still with $J_{x}=J_{y} \equiv J_{\perp}$. It is important to note that this is a four-channel model: in each of the two leads, 
electrons are transported in both directions. This is different from the threedimensional single impurity model, for which radial symmetry is used to map each lead to a single one-dimensional channel. However, we also note that the above Hamiltonian only includes forward scattering of the electrons on the impurities; in presence of backscattering (which can either be a relevant or an irrelevant perturbation [75, 76]), left- and right-movers can turn into each other by bouncing off the impurities. In this case, the left- and right-moving modes on a given lead are therefore coupled to each other through impuritymediated cotunnelling. Following the discussion from Sec. 3.1, these two channels thus combine into a single effective channel at low temperatures, such that the full model reduces to a two-channel model. Nevertheless, the one-dimensional two-lead Kondo lattice provides a way to study novel fourchannel Kondo physics whenever backscattering is suppressed.

The single lead version of the above Kondo lattice model has previously been studied by Zachar, Kivelson and Emery in Ref. [75]. One of their main results was that the ground state can exhibit antiferromagnetic ordering in the Kondo impurities, despite being a complicated NFL two-channel setup. Inspired by their work, our first step towards understanding the behaviour of the two-lead model is to bosonize it, using the bosonization identities

$$
\begin{gathered}
\psi_{\eta \alpha \sigma}(x)=\frac{1}{\sqrt{2 \pi a_{0}}} e^{-i \varphi_{\eta \alpha \sigma}(x)}, \quad \psi_{\eta \alpha \sigma}^{\dagger}(x)=\frac{1}{\sqrt{2 \pi a_{0}}} e^{i \varphi_{\eta \alpha \sigma}(x)}, \\
\varphi_{\eta \alpha \sigma}(x)=\sqrt{\pi}\left[\Theta_{\alpha \sigma}(x) \pm \Phi_{\alpha \sigma}(x)\right] \pm k_{F} x, \quad \Theta_{\alpha \sigma}(x)=\int_{-\infty}^{x} \mathrm{~d} x^{\prime} \Pi_{\alpha \sigma}\left(x^{\prime}\right),
\end{gathered}
$$

where the plus and minus signs respectively correspond to $\eta=l$ and $\eta=r$, and the bosonic fields $\Phi_{\alpha \sigma}(x)$ and $\Pi_{\alpha \sigma}(x)$ are chosen to be conjugate. At the equivalent of the EK point (in this case $J_{z}=\pi \hbar v_{F}$ ), the bosonization and subsequent unitary transformation result in

$$
\begin{aligned}
& \hat{H}=\frac{\hbar v_{F}}{2} \sum_{\nu} \int_{-\infty}^{\infty} \mathrm{d} x\left[\left(\Pi_{\nu}(x)\right)^{2}+\left(\partial_{x} \Phi_{\nu}(x)\right)^{2}\right]+\sum_{j} \frac{2 J_{\perp}}{\pi a_{0}} \\
& \times\left[\tau_{j}^{x} \cos \left(\sqrt{\pi} \Phi_{s}\left(x_{j}\right)+\pi \sum_{m} \theta\left(x_{m}-x_{j}\right) \tau_{m}^{z}\right) \cos \left(\sqrt{\pi} \Theta_{s f}\left(x_{j}\right)\right) \cos \left(\sqrt{\pi} \Phi_{s f}\left(x_{j}\right)\right)\right. \\
& \left.-\tau_{j}^{y} \sin \left(\sqrt{\pi} \Phi_{s}\left(x_{j}\right)+\pi \sum_{m} \theta\left(x_{m}-x_{j}\right) \tau_{m}^{z}\right) \sin \left(\sqrt{\pi} \Theta_{s f}\left(x_{j}\right)\right) \sin \left(\sqrt{\pi} \Phi_{s f}\left(x_{j}\right)\right)\right],
\end{aligned}
$$


where again $\nu=c, s, f, s f$. When compared to the single lead model from Ref. [75], for which the coupling terms can similarly be mapped to

$$
\begin{aligned}
\frac{J_{\perp}}{\pi a_{0}} \sum_{j} \tau_{j}^{x} \cos \left(\sqrt{2 \pi} \Phi_{s}(j)+2 \pi \sum_{m} \theta\left(x_{m}-x_{j}\right) \tau_{m}^{z}\right) \\
=\frac{J_{\perp}}{\pi a_{0}} \sum_{j}(-1)^{j} \tau_{j}^{x} \cos \left(\sqrt{2 \pi} \Phi_{s}(j)\right),
\end{aligned}
$$

there are some significant differences. Most importantly, the prefactor of the step function is only half the size of the corresponding one-lead analogue, such that these terms result in phase shifts of $\pm \pi / 2$ between the lattice sites, instead of $\pm \pi$. As such, there is no alternating coupling, and the explicit $\tau_{j}^{z}$-dependence remains important. The unitary transformation required to remove $\tau_{j}^{x}$ or $\tau_{j}^{y}$ from the Hamiltonian thus necessarily depends on $\Phi_{s}\left(x_{j}\right), \Theta_{s f}\left(x_{j}\right), \Phi_{s f}\left(x_{j}\right)$ and $\tau_{j}^{z}$, and as a result there exists no unitary transformation that removes $\tau_{j}^{x}$ or $\tau_{j}^{y}$ without also introducing an even more complicated $\tau_{j}^{z}$-dependence. It therefore also follows that there is no component of the spin that commutes with the Hamiltonian. Consequently, the two-lead model does not have an antiferromagnetically ordered ground state, contrary to the one-lead model. Secondly, we observe that some of the bosonic fields remain coupled to each other, preventing refermionization to a non-interacting model. However, the single impurity four-channel Kondo model can also not be refermionized to a non-interacting model (in that case due to the scaling dimension of the boundary cosine operator), but can still be studied to a large extend by exploiting the fact that the refermionized model is equivalent to an impurity in the well-known interacting Luttinger liquid [51, 77, 78]. It is therefore reasonable to suspect that a similar strategy will also work for the two-lead Kondo lattice, providing a solid starting point for studying this four-channel lattice model.

\subsection{Outlook}

Following the extensions discussed in this chapter, we conclude with a summary of the possible future directions for research on multichannel Kondo physics. On a theoretical level, the most obvious next step is to make (possibly numerical) predictions regarding heat transport along the whole FL crossover, or at least for the lowest order corrections to the heat conductance in the FL regime. Additionally, the same methods can be applied in an attempt to qualitatively derive the lowest order corrections to the heat 
conductance as a function of $T / T_{K}$. Such results would offer further insight into the transition from the local moment fixed point to the NFL critical point, in particular concerning the Wiedemann-Franz law and the emergence of the Majorana degrees of freedom. To complement these theoretical predictions, it would be interesting to see experimental heat transport measurements on the C2CK device along the entire universal scaling curve; this would further establish our current understanding of $2 \mathrm{CK}$ physics and our conclusions regarding the Wiedemann-Franz law, while providing a direct experimental verification of the elusive Majorana nature of the excitations at the NFL critical point. Finally, it would also be interesting to extend our study to three- and four-channel Kondo systems, such as the lattice model from Sec. 8.3 and the charge three-channel Kondo setup. The latter is very challenging from a theoretical perspective: the three-channel Kondo model does not have a Toulouse point (such that it cannot be solved exactly), and perturbation theory cannot be applied because it breaks down at the three-channel NFL critical point. However, a three-channel version of the C2CK setup has recently been realized as well, such that three-channel heat transport measurements are also within experimental reach [71]. 



\section{Appendix}

\section{A.1 Bosonization}

In this section, we will derive the bosonization formulas used in Sec. 4.2.2, following Refs. [50, 51. The first realization necessary to derive these relations is that the dispersion relation has been linearized around the Fermi level, such that the one-dimensional fermionic fields are only allowed to move with constant velocity $v_{F}$ in either direction. Introducing coordinates $z=i\left(x+v_{F} t\right)=v_{F} \tau+i x$ and $\bar{z}=-i\left(x-v_{F} t\right)=v_{F} \tau-i x$, the fields can be divided into left-movers $\psi(x, t)=\psi(z)$ and right-movers $\bar{\psi}(x, t)=\bar{\psi}(\bar{z})$. Since all fermionic fields in the anisotropic $2 \mathrm{CK}$ model are left-movers, we will now only consider fields of the form $\psi(z)$, noting that $\partial_{x}=i\left(\partial_{z}-\partial_{\bar{z}}\right)=i \partial_{z}$ when acting on these fields.

The bosonization ansatz is that one can introduce a bosonic left-moving field $\Phi(z)$ that is related to its fermionic counterpart through the relations

$$
\psi(z)=A \eta e^{-i \lambda \Phi(z)}, \quad \psi^{\dagger}(z)=A \eta e^{i \lambda \Phi(z)},
$$

where $A$ and $\lambda$ are real positive constants, and $\eta$ is a Klein factor that is introduced to ensure the anticommutation of different types of fermions (i.e. $\left\{\eta_{i}, \eta_{j}\right\}=2 \delta_{i, j}$ for fermion species $i$ and $j$ ). The operator exponentials in the above expressions are understood as normal ordered. In order to work with normal ordered operator exponentials of this form, we take notice of a useful formula:

$$
: e^{i \alpha \Phi(z)}:: e^{i \beta \Phi\left(z^{\prime}\right)}:=: e^{i \alpha \Phi(z)+i \beta \Phi\left(z^{\prime}\right)}: e^{-\alpha \beta\left\langle\Phi(z) \Phi\left(z^{\prime}\right)\right\rangle},
$$

which is a direct consequence of the Campbell-Baker-Hausdorff formula (see e.g. Ref. [50]). Furthermore, the fermionic and bosonic two-point functions can be calculated by performing mode expansions of the fields 11 leading to

$$
\left\langle\psi^{\dagger}(z) \psi\left(z^{\prime}\right)\right\rangle=\frac{1}{2 \pi} \frac{1}{z-z^{\prime}}, \quad\left\langle\Phi(z) \Phi\left(z^{\prime}\right)\right\rangle=\ln \left(\frac{a_{0}}{z-z^{\prime}}\right)
$$

\footnotetext{
${ }^{1}$ The same mode expansions can also be used to derive the correct (anti)commutation relations for the fields, including the one from Eq. 4.19.
} 
for $\tau>\tau^{\prime} ; a_{0}$ is a small regularization parameter that must be included in the mode expansion of the bosonic fields, and is often taken to be of the order of the lattice spacing of the lattice on which the calculation was performed [78. Combining all of the above equations and writing the Klein factor as an exponential, the desired bosonization identity is found to be

$$
\psi(z)=\frac{1}{\sqrt{2 \pi a_{0}}} e^{i \phi} e^{-i \Phi(z)},
$$

which is the identity used in the Emery-Kivelson mapping procedure.

In the remainder of this section, we will use the above to derive Eqs. (4.15), (4.16) and (4.21) from the main text. To do so, we make use of Wick's theorem, stating that a normal ordered product is equal to the corresponding time ordered product minus all possible contractions. For equal-time products, the use of time ordering and two-point functions requires pointsplitting, which we implement by evaluating all creation operators at an imaginary time $\delta / v_{F}$ later than the annihilation operators. First considering a density term:

$$
\begin{aligned}
: \psi^{\dagger}(z+\delta) \psi(z): & =T_{\tau} \psi^{\dagger}(z+\delta) \psi(z)-\left\langle\psi^{\dagger}(z+\delta) \psi(z)\right\rangle \\
& =\frac{1}{2 \pi a_{0}}: e^{i \Phi(z+\delta)-i \Phi(z)}: e^{\langle\Phi(z+\delta) \Phi(z)\rangle}-\frac{1}{2 \pi \delta} \\
& =\frac{1}{2 \pi \delta}\left(: e^{i \partial_{z} \Phi(z) \delta+\mathcal{O}\left(\delta^{2}\right)}:-1\right) \\
& =\frac{i}{2 \pi} \partial_{z} \Phi(z)+\mathcal{O}(\delta),
\end{aligned}
$$

Taking the limit $\delta \rightarrow 0$ and using $i \partial_{z}=\partial_{x}$, this immediately leads to Eq. (4.15). Evaluation of kinetic terms is more subtle, and we find them by considering an overall derivative instead:

$$
\begin{aligned}
& : \psi^{\dagger}(z+\delta) \partial_{z} \psi(z): \\
& \quad=\partial_{z}: \psi^{\dagger}\left(z^{\prime}\right) \psi(z):||_{z^{\prime} \rightarrow z+\delta} \\
& \quad=\left.\partial_{z}\left[\frac{1}{2 \pi} \frac{1}{z^{\prime}-z}\left(: e^{i \Phi\left(z^{\prime}\right)-i \Phi(z)}:-1\right)\right]\right|_{z^{\prime} \rightarrow z+\delta} \\
& \quad=\left.\partial_{z}\left[\frac{1}{2 \pi} \frac{1}{z^{\prime}-z}\left(i\left(\Phi\left(z^{\prime}\right)-\Phi(z)\right)-\frac{1}{2}\left(\Phi\left(z^{\prime}\right)-\Phi(z)\right)^{2}+\ldots\right)\right]\right|_{z^{\prime} \rightarrow z+\delta} \\
& \quad=\frac{i}{4 \pi} \partial_{z}^{2} \Phi(z)+\frac{1}{4 \pi}\left(\partial_{z} \Phi(z)\right)^{2}+\mathcal{O}(\delta) .
\end{aligned}
$$


The final line of this equation is obtained by first evaluating the derivative, then replacing $z^{\prime} \rightarrow z+\delta$ and expanding the result in $\delta$. Integrating over $x$ and again taking the limit $\delta \rightarrow 0$, we find

$$
\begin{aligned}
\int_{-\infty}^{\infty} \mathrm{d} x: \psi^{\dagger}(x) \partial_{x} \psi(x): & =-\frac{i}{4 \pi} \int_{-\infty}^{\infty} \mathrm{d} x\left(\partial_{x} \Phi(x)\right)^{2}+\left.\frac{1}{4 \pi} \partial_{x} \Phi(x)\right|_{-\infty} ^{\infty} \\
& =-\frac{i}{4 \pi} \int_{-\infty}^{\infty} \mathrm{d} x\left(\partial_{x} \Phi(x)\right)^{2}+\frac{1}{2}: \psi^{\dagger}(x) \psi(x):\left.\right|_{-\infty} ^{\infty} .
\end{aligned}
$$

The boundary term on the right-hand side is equal to the difference in density at both ends of the infinite one-dimensional system and therefore equal to zero, such that the above is the same as Eq. (4.16). Finally, the bosonization of interaction terms is a straightforward extension of the bosonization of density terms. Distinguishing between two different fermion species $\alpha \neq \beta$ such that $\psi_{\alpha}(z)$ and $\psi_{\beta}^{\dagger}\left(z^{\prime}\right)$ anticommute, and $\Phi_{\alpha}(z)$ and $\Phi_{\beta}\left(z^{\prime}\right)$ commute, we simply find

$$
\begin{aligned}
: \psi_{\alpha}^{\dagger}(z+\delta) \psi_{\alpha}(z) \psi_{\beta}^{\dagger}(z+\delta) \psi_{\beta}(z): & =: \psi_{\alpha}^{\dagger}(z+\delta) \psi_{\alpha}(z):: \psi_{\beta}^{\dagger}(z+\delta) \psi_{\beta}(z): \\
& =-\frac{1}{4 \pi^{2}}\left(\partial_{z} \Phi_{\alpha}(z)\right)\left(\partial_{z} \Phi_{\beta}(z)\right)+\mathcal{O}(\delta)
\end{aligned}
$$

Similar to the previous bosonization formulas, this straightforwardly leads to Eq. 4.21.

\section{A.2 Majorana Green functions on the dot}

In the following, we will calculate the necessary components of the dot Green function $\mathbf{D}\left(t, t^{\prime}\right)$ in presence of a bias voltage. Referring back to the selfenergy from Eq. 4.35) and the Hamiltonian from Eq. 4.44, and using structures from Eqs. (4.31) and 4.32), we find

$$
\begin{aligned}
\mathbf{D} & =\left(\mathbf{D}_{0}^{-1}-\frac{g_{\perp}^{2}}{\hbar^{2}} \mathbf{g}^{\dagger} \cdot \mathbf{L}_{0}^{\prime} \cdot \mathbf{g}\right)^{-1} \\
& =\left(\left.\mathbf{D}_{0}^{-1}\right|_{B=0}-\frac{B}{\hbar} \delta\left(t-t^{\prime}\right)\left(\begin{array}{cc}
1 & 0 \\
0 & -1
\end{array}\right)-\frac{g_{\perp}^{2}}{\hbar^{2}}\left(L_{0,1}^{\prime}+L_{0,2}^{\prime}\right)\left(\begin{array}{cc}
1 & -1 \\
-1 & 1
\end{array}\right)\right)^{-1} .
\end{aligned}
$$


Let us now look at the retarded and advanced components of this Green function, using Eq. (5.4):

$$
\begin{aligned}
\mathbf{D}^{\mathrm{R} / \mathrm{A}}\left(t, t^{\prime}\right) & =\left(\delta\left(t-t^{\prime}\right)\left(\begin{array}{cc}
i \partial_{t^{\prime}}-\frac{B}{\hbar} \pm \frac{i g_{\perp}^{2}}{\hbar^{2} v_{F}} & \mp \frac{i g_{\perp}^{2}}{\hbar^{2} v_{F}} \\
\mp \frac{i g_{\perp}^{2}}{\hbar^{2} v_{F}} & i \partial_{t^{\prime}}+\frac{B}{\hbar} \pm \frac{i g_{\perp}^{2}}{\hbar^{2} v_{F}}
\end{array}\right)\right)^{-1} \\
& =\left(\delta\left(t-t^{\prime}\right)\left(\begin{array}{cc}
i \partial_{t^{\prime}} \pm \frac{1}{\hbar}\left(\mp B+\frac{i}{2} \Gamma\right) & \mp \frac{i \Gamma}{2 \hbar} \\
\mp \frac{i \Gamma}{2 \hbar} & i \partial_{t^{\prime}} \pm \frac{1}{\hbar}\left( \pm B+\frac{i}{2} \Gamma\right)
\end{array}\right)\right)^{-1},
\end{aligned}
$$

where the convergence factor $i 0^{+}$has been omitted by virtue of the presence of a non-zero imaginary part. As seen in the main text, it is convenient to consider the Fourier transform of the dot Green function as well. In particular, we work out the following object, which also appears in the expressions for the currents:

$$
\begin{aligned}
\mathbf{D}^{\mathrm{R} / \mathrm{A}}(\epsilon) & =\int_{-\infty}^{\infty} \mathrm{d} \Delta t e^{\frac{i e}{\hbar} \Delta t} \mathbf{D}^{\mathrm{R} / \mathrm{A}}(\Delta t) \\
& =\int_{-\infty}^{\infty} \mathrm{d} t^{\prime} e^{-\frac{i \epsilon}{\hbar}\left(t-t^{\prime}\right)} \mathbf{D}^{\mathrm{R} / \mathrm{A}}\left(t^{\prime}, t\right),
\end{aligned}
$$

where we used that $\mathbf{D}^{\mathrm{R} / \mathrm{A}}\left(t, t^{\prime}\right)=\mathbf{D}^{\mathrm{R} / \mathrm{A}}\left(t-t^{\prime}\right)$, while in the second line we wrote $\Delta t \equiv t^{\prime}-t$, with $t$ a constant. This is allowed due to the fact that the time-dependence of the Hamiltonian does not influence any of the necessary components (see again Eq. (5.4) ) and can therefore be treated as fully time-independent. First calculating the Fourier transform of the inverse of $\mathbf{D}^{\mathrm{R} / \mathrm{A}}\left(t, t^{\prime}\right)$ :

$$
\begin{aligned}
\left(\mathbf{D}^{\mathrm{R} / \mathrm{A}}\right)^{-1}(\epsilon) & \\
\quad= & \int_{-\infty}^{\infty} \mathrm{d} t^{\prime} \delta\left(t^{\prime}-t\right)\left(\begin{array}{cc}
i \partial_{t} \pm \frac{1}{\hbar}\left(\mp B+\frac{i}{2} \Gamma\right) & \mp \frac{i \Gamma}{2 \hbar}
\end{array} \underset{\partial_{t} \pm \frac{1}{\hbar}\left( \pm B+\frac{i}{2 \hbar} \Gamma\right)}{\mp}\right) e^{-\frac{i \epsilon}{\hbar}\left(t-t^{\prime}\right)} \\
& =\frac{1}{\hbar}\left(\begin{array}{cc}
\epsilon-B \pm \frac{i}{2} \Gamma & \mp \frac{i}{2} \Gamma \\
\mp \frac{i}{2} \Gamma & \epsilon+B \pm \frac{i}{2} \Gamma
\end{array}\right)
\end{aligned}
$$

Inverting this matrix, we obtain

$$
\mathbf{D}^{\mathrm{R} / \mathrm{A}}(\epsilon)=\frac{\hbar}{\epsilon(\epsilon \pm i \Gamma)-B^{2}}\left(\begin{array}{cc}
\epsilon+B \pm \frac{i}{2} \Gamma & \pm \frac{i}{2} \Gamma \\
\pm \frac{i}{2} \Gamma & \epsilon-B \pm \frac{i}{2} \Gamma
\end{array}\right)
$$


Referring back to Eqs. 4.36)-(4.39), it also immediately follows that the Fourier transformed Majorana Green functions are given by

$$
\begin{aligned}
D_{a a}^{\mathrm{R} / \mathrm{A}}(\epsilon) & =\frac{\hbar(\epsilon \pm i \Gamma)}{\epsilon(\epsilon \pm i \Gamma)-B^{2}}, \\
D_{b b}^{\mathrm{R} / \mathrm{A}}(\epsilon) & =\frac{\hbar \epsilon}{\epsilon(\epsilon \pm i \Gamma)-B^{2}}, \\
D_{a b}^{\mathrm{R} / \mathrm{A}}(\epsilon) & =\frac{-i \hbar B}{\epsilon(\epsilon \pm i \Gamma)-B^{2}}=-D_{b a}^{\mathrm{R} / \mathrm{A}}(\epsilon) .
\end{aligned}
$$

Although in some cases (such as the dc and the ac cases from the main text) it suffices to know the Fourier transform of the dot Green function, it is often necessary to have an expression for $\mathbf{D}^{\mathrm{R} / \mathrm{A}}\left(t, t^{\prime}\right)$ itself. This expression can be found by evaluating the following integral:

$$
\mathbf{D}^{\mathrm{R} / \mathrm{A}}\left(t, t^{\prime}\right)=\int_{-\infty}^{\infty} \frac{\mathrm{d} \epsilon}{2 \pi \hbar} e^{-\frac{i \epsilon}{\hbar}\left(t-t^{\prime}\right)} \frac{\hbar}{\epsilon(\epsilon \pm i \Gamma)-B^{2}}\left(\begin{array}{cc}
\epsilon+B \pm \frac{i}{2} \Gamma & \pm \frac{i}{2} \Gamma \\
\pm \frac{i}{2} \Gamma & \epsilon-B \pm \frac{i}{2} \Gamma
\end{array}\right) .
$$

We evaluate this integral using contour integration. The poles of the integrand are located at $\epsilon_{ \pm, n}=\mp i \Gamma / 2+(-1)^{n} \sqrt{B^{2}-\Gamma^{2} / 4}=\mp i(\Gamma / 2 \mp$ $(-1)^{n} \sqrt{\Gamma^{2} / 4-B^{2}}$ ) (where $n=0,1$ ), so in the case of the retarded (advanced) component, they are both located in the lower (upper) half of the imaginary plane for any real $B$. Properly closing the contour, we thus find that $\mathbf{D}^{\mathrm{R}(\mathrm{A})}\left(t, t^{\prime}\right)$ is zero if $t<t^{\prime}\left(t>t^{\prime}\right)$, and non-zero otherwise. This confirms that it is indeed a retarded (advanced) function. Meanwhile, the denominator in the integrand can be written as $(-1)^{n} i \sqrt{\Gamma^{2}-4 B^{2}}\left(\epsilon-\epsilon_{ \pm, n}\right)$ to linear order around the poles. Applying the residue theorem:

$$
\begin{aligned}
\mathbf{D}^{\mathrm{R} / \mathrm{A}}\left(t, t^{\prime}\right)= & \mp i \theta\left( \pm\left(t-t^{\prime}\right)\right) \\
& \times \sum_{n} \operatorname{Res}\left(\frac{e^{-\frac{i \epsilon}{\hbar}\left(t-t^{\prime}\right)}}{\epsilon(\epsilon \pm i \Gamma)-B^{2}}\left(\begin{array}{cc}
\epsilon+B \pm \frac{i}{2} \Gamma & \pm \frac{i}{2} \Gamma \\
\pm \frac{i}{2} \Gamma & \epsilon-B \pm \frac{i}{2} \Gamma
\end{array}\right), \epsilon_{ \pm, n}\right) \\
= & \mp i \theta\left( \pm\left(t-t^{\prime}\right)\right) \frac{-i}{\sqrt{\Gamma^{2}-4 B^{2}}} \\
& \times\left[e^{-\frac{i \epsilon_{ \pm}, 0}{\hbar}\left(t-t^{\prime}\right)}\left(\begin{array}{cc}
\epsilon_{ \pm, 0}+B \pm \frac{i}{2} \Gamma & \pm \frac{i}{2} \Gamma \\
\pm \frac{i}{2} \Gamma & \epsilon_{ \pm, 0}-B \pm \frac{i}{2} \Gamma
\end{array}\right)\right. \\
& \left.-e^{-\frac{i \epsilon_{ \pm, 1}}{\hbar}\left(t-t^{\prime}\right)}\left(\begin{array}{cc}
\epsilon_{ \pm, 1}+B \pm \frac{i}{2} \Gamma & \pm \frac{i}{2} \Gamma \\
\pm \frac{i}{2} \Gamma & \epsilon_{ \pm, 1}-B \pm \frac{i}{2} \Gamma
\end{array}\right)\right],
\end{aligned}
$$


where the overall sign in front emerges from the integration direction. Writing out the components of the above matrix, we find

$$
\begin{aligned}
D_{11}^{\mathrm{R} / \mathrm{A}}\left(t, t^{\prime}\right)= & \mp i \theta\left( \pm\left(t-t^{\prime}\right)\right) e^{\mp \frac{\Gamma}{2 \hbar}\left(t-t^{\prime}\right)}\left[\cosh \left(\frac{1}{2 \hbar} \sqrt{\Gamma^{2}-4 B^{2}}\left(t-t^{\prime}\right)\right)\right. \\
& \left.-\frac{2 i B}{\sqrt{\Gamma^{2}-4 B^{2}}} \sinh \left(\frac{1}{2 \hbar} \sqrt{\Gamma^{2}-4 B^{2}}\left(t-t^{\prime}\right)\right)\right], \\
D_{12}^{\mathrm{R} / \mathrm{A}}\left(t, t^{\prime}\right)= & -i \theta\left( \pm\left(t-t^{\prime}\right)\right) e^{\mp \frac{\Gamma}{2 \hbar}\left(t-t^{\prime}\right)} \\
& \times \frac{\Gamma}{\sqrt{\Gamma^{2}-4 B^{2}}} \sinh \left(\frac{1}{2 \hbar} \sqrt{\Gamma^{2}-4 B^{2}}\left(t-t^{\prime}\right)\right),
\end{aligned}
$$

and

$$
D_{21}^{\mathrm{R} / \mathrm{A}}\left(t, t^{\prime}\right)=D_{12}^{\mathrm{R} / \mathrm{A}}\left(t, t^{\prime}\right), \quad D_{22}^{\mathrm{R} / \mathrm{A}}\left(t, t^{\prime}\right)=\left.D_{11}^{\mathrm{R} / \mathrm{A}}\left(t, t^{\prime}\right)\right|_{B \rightarrow-B} .
$$

Meanwhile, the Majorana Green functions are given by

$$
\begin{aligned}
D_{a a}^{\mathrm{R} / \mathrm{A}}\left(t, t^{\prime}\right)= & \mp i \theta\left( \pm\left(t-t^{\prime}\right)\right) e^{\mp \frac{\Gamma}{2 \hbar}\left(t-t^{\prime}\right)}\left[\cosh \left(\frac{1}{2 \hbar} \sqrt{\Gamma^{2}-4 B^{2}}\left(t-t^{\prime}\right)\right)\right. \\
& \left. \pm \frac{\Gamma}{\sqrt{\Gamma^{2}-4 B^{2}}} \sinh \left(\frac{1}{2 \hbar} \sqrt{\Gamma^{2}-4 B^{2}}\left(t-t^{\prime}\right)\right)\right], \\
D_{b b}^{\mathrm{R} / \mathrm{A}}\left(t, t^{\prime}\right)= & \mp i \theta\left( \pm\left(t-t^{\prime}\right)\right) e^{\mp \frac{\Gamma}{2 \hbar}\left(t-t^{\prime}\right)}\left[\cosh \left(\frac{1}{2 \hbar} \sqrt{\Gamma^{2}-4 B^{2}}\left(t-t^{\prime}\right)\right)\right. \\
& \left.\mp \frac{\Gamma}{\sqrt{\Gamma^{2}-4 B^{2}}} \sinh \left(\frac{1}{2 \hbar} \sqrt{\Gamma^{2}-4 B^{2}}\left(t-t^{\prime}\right)\right)\right], \\
D_{a b}^{\mathrm{R} / \mathrm{A}}\left(t, t^{\prime}\right)= & \pm i \theta\left( \pm\left(t-t^{\prime}\right)\right) e^{\mp \frac{\Gamma}{2 \hbar}\left(t-t^{\prime}\right)} \\
& \times \frac{2 B}{\sqrt{\Gamma^{2}-4 B^{2}}} \sinh \left(\frac{1}{2 \hbar} \sqrt{\Gamma^{2}-4 B^{2}}\left(t-t^{\prime}\right)\right),
\end{aligned}
$$

and $D_{b a}^{\mathrm{R} / \mathrm{A}}\left(t, t^{\prime}\right)=-D_{a b}^{\mathrm{R} / \mathrm{A}}\left(t, t^{\prime}\right)$. For future reference, note in particular that $D_{b a}^{\mathrm{R} / \mathrm{A}}\left(t, t^{\prime}\right)$ is proportional to the magnetic field $B$. This remains true upon analytically continuing to imaginary time $\tau$, such that the time-ordered expectation value $\left\langle T_{\tau} b(\tau) a\left(\tau^{\prime}\right)\right\rangle$ goes to zero with the magnetic field.

\section{A.3 Notes on the Keldysh structure}

In the case of fermions, the Keldysh formalism for non-equilibrium problems [47] starts with the action as a functional of conjugate Grassmann 
fields $\psi$ and $\bar{\psi}$, involving integration over the closed time contour $\mathcal{C}$ (which consists of a forward branch from $-\infty$ to $+\infty$, and a backward branch from $+\infty$ to $-\infty)$. The fields are then doubled: one field for the forward branch, and one for the backward branch. Following the Keldysh prescription of doubling and rotating the fields (such that we have two new fields $\psi_{1}$ and $\psi_{2}$ for each field $\psi$ ), the action becomes

$$
S=\hbar \int_{-\infty}^{\infty} \mathrm{d} t \bar{\psi} \cdot G^{-1} \otimes \gamma^{\mathrm{cl}} \cdot \psi,
$$

with

$$
\psi \equiv\left(\psi_{1} \psi_{2}\right)^{T}, \quad \gamma^{\mathrm{cl}} \equiv\left(\begin{array}{cc}
1 & 0 \\
0 & 1
\end{array}\right) .
$$

In this Keldysh rotated (1,2)-basis, the full Green function $\mathbf{G}$ assumes a triangular structure:

$$
\mathbf{G}=\left(\begin{array}{cc}
G^{\mathrm{R}} & G^{\mathrm{K}} \\
0 & G^{\mathrm{A}}
\end{array}\right)
$$

Here, $G^{\mathrm{R} / \mathrm{A}}$ are the usual retarded and advanced Green functions, while $G^{\mathrm{K}}$ is referred to as the Keldysh component of the Green function. In general, expectation values relate to these components in the following way:

$$
\left\langle\psi_{\alpha} \psi_{\beta}^{\dagger}\right\rangle=\frac{1}{2}\left\langle\psi_{\alpha, 1} \bar{\psi}_{\beta, 2}\right\rangle=\frac{i}{2} G_{\alpha \beta}^{\mathrm{K}},
$$

where the labels $\alpha, \beta$ have been added to distinguish between the different fields of a multicomponent system, such as the leads and the dot of a quantum dot device.

As discussed in the main text, the Keldysh Green function of a system in thermal equilibrium can be found by using the FDT. However, the dot region is not in equilibrium, so the Keldysh component on the dot has to be found in a different way. In order to find this component, we apply block inversion to the triangular Keldysh structure. This gives

$$
\begin{gathered}
\left(\mathbf{G}^{-1}\right)^{\mathrm{R} / \mathrm{A}}=\left(\mathbf{G}^{\mathrm{R} / \mathrm{A}}\right)^{-1}, \\
\left(\mathbf{G}^{-1}\right)^{\mathrm{K}}=-\left(\mathbf{G}^{\mathrm{R}}\right)^{-1} \cdot \mathbf{G}^{\mathrm{K}} \cdot\left(\mathbf{G}^{\mathrm{A}}\right)^{-1},
\end{gathered}
$$

which can be combined to find

$$
\mathbf{D}^{\mathrm{K}}=-\mathbf{D}^{\mathrm{R}} \cdot\left(\left(\mathbf{D}_{0}^{-1}\right)^{\mathrm{K}}-\boldsymbol{\Sigma}_{d}^{\mathrm{K}}\right) \cdot \mathbf{D}^{\mathrm{A}}
$$


for the Keldysh component of the full Green function on the dot. Furthermore combining Eqs. 4.52), 4.53 and A.30 with the general properties of the (bare) retarded and advanced Green functions, we see that the term

$$
\left(\mathbf{D}_{0}^{-1}\right)^{\mathrm{K}}=2 i 0^{+} \mathbf{F}
$$

is just a regulator. The above Keldysh component therefore reduces to

$$
\mathbf{D}^{\mathrm{K}}=\mathbf{D}^{\mathrm{R}} \cdot \Sigma_{d}^{\mathrm{K}} \cdot \mathbf{D}^{\mathrm{A}},
$$

eliminating the need to find the out-of-equilibrium matrix $\mathbf{F}$ on the dot.

Another thing to note is that the FDT from Eq. 4.53) should in general be applied to Wigner transformed Green functions. To see why the FDT manifests itself in the way that it does in Sec. 5.1, consider a simple timeindependent and homogeneous model. In this case, the Wigner transform simply reduces to the usual Fourier transform. Applying the FDT:

$$
\begin{aligned}
G_{0, k}^{\mathrm{K}}(\epsilon) & =f(\epsilon)\left(G_{0, k}^{\mathrm{R}}(\epsilon)-G_{0, k}^{\mathrm{A}}(\epsilon)\right) \\
& =f(\epsilon)\left(\frac{\hbar}{\epsilon-\epsilon_{k}+i 0^{+}}-\frac{\hbar}{\epsilon-\epsilon_{k}-i 0^{+}}\right) \\
& =-2 i \hbar f(\epsilon) \frac{0^{+}}{\left(\epsilon-\epsilon_{k}\right)^{2}+\left(0^{+}\right)^{2}} \\
& =-2 \pi i \hbar f(\epsilon) \delta\left(\epsilon-\epsilon_{k}\right) .
\end{aligned}
$$

Here, $\epsilon_{k}$ is a function of the wave number $k$, while the energy $\epsilon$ is the variable conjugate to the time $t$. Fourier transforming the the energy back to time:

$$
\begin{aligned}
G_{0, k}^{\mathrm{K}}\left(t, t^{\prime}\right) & =\int_{-\infty}^{\infty} \frac{\mathrm{d} \epsilon}{2 \pi \hbar} G_{0, k}^{\mathrm{K}}(\epsilon) e^{-\frac{i \epsilon}{\hbar}\left(t-t^{\prime}\right)} \\
& =-i f\left(\epsilon_{k}\right) e^{-\frac{i \epsilon_{k}}{\hbar}\left(t-t^{\prime}\right)} .
\end{aligned}
$$

To obtain the additional time-dependence due to the bias voltage as seen in Eq. (5.5), we subsequently return to the assumption that the bias voltage only acts on the junction without influencing the flavour and spin-flavour modes themselves (i.e. keeping $f\left(\epsilon_{k}\right)$ invariant), such that it can be incorporated by replacing the $\epsilon_{k}$ in the exponent with $\epsilon_{k}-e V(t) / 2$ [52].

\section{A.4 Kubo formula}

The Kubo formula from Eq. 4.56 can be obtained by using functional integral formalism in imaginary time $\tau$. The contribution of the potential 
drop to the Euclidean action is $S_{\phi}=\int_{0}^{\hbar \beta} \mathrm{d} \tau \hat{H}_{\phi}(\tau)=\int_{0}^{\hbar \beta} \mathrm{d} \tau \hat{Q}(\tau) \Delta \phi(\tau)$, from where it follows that

$$
\begin{aligned}
\left\langle\hat{I}_{Q}\right\rangle(\tau)= & \frac{1}{Z} \int \mathcal{D} \psi \hat{I}_{Q}(\tau) e^{-\frac{S_{0}}{\hbar}-\frac{S_{\phi}}{\hbar}} \\
= & \frac{\int \mathcal{D} \psi \hat{I}_{Q}(\tau)\left(1-\frac{1}{\hbar} \int_{0}^{\hbar \beta} \mathrm{d} \tau^{\prime} \hat{Q}\left(\tau^{\prime}\right) \Delta \phi\left(\tau^{\prime}\right)+\mathcal{O}\left(\Delta \phi^{2}\right)\right) e^{-\frac{S_{0}}{\hbar}}}{\int \mathcal{D} \psi\left(1-\frac{1}{\hbar} \int_{0}^{\hbar \beta} \mathrm{d} \tau^{\prime} \hat{Q}\left(\tau^{\prime}\right) \Delta \phi\left(\tau^{\prime}\right)+\mathcal{O}\left(\Delta \phi^{2}\right)\right) e^{-\frac{S_{0}}{\hbar}}} \\
= & \frac{\int \mathcal{D} \psi \hat{I}_{Q}(\tau)\left(1-\frac{1}{\hbar} \int_{0}^{\hbar \beta} \mathrm{d} \tau^{\prime} \hat{Q}\left(\tau^{\prime}\right) \Delta \phi\left(\tau^{\prime}\right)+\mathcal{O}\left(\Delta \phi^{2}\right)\right) e^{-\frac{S_{0}}{\hbar}}}{Z_{0}\left(1-\frac{1}{\hbar} \int_{0}^{\hbar \beta} \mathrm{d} \tau^{\prime}\left\langle\hat{Q}\left(\tau^{\prime}\right)\right\rangle_{0} \Delta \phi\left(\tau^{\prime}\right)+\mathcal{O}\left(\Delta \phi^{2}\right)\right)} \\
= & \left\langle\hat{I}_{Q}(\tau)\right\rangle_{0}-\frac{1}{\hbar} \int_{0}^{\hbar \beta} \mathrm{d} \tau^{\prime}\left\langle T_{\tau} \hat{I}_{Q}(\tau) \hat{Q}\left(\tau^{\prime}\right)\right\rangle_{0} \Delta \phi\left(\tau^{\prime}\right) \\
& +\frac{1}{\hbar} \int_{0}^{\hbar \beta} \mathrm{d} \tau^{\prime}\left\langle\hat{I}_{Q}(\tau)\right\rangle_{0}\left\langle\hat{Q}\left(\tau^{\prime}\right)\right\rangle_{0} \Delta \phi\left(\tau^{\prime}\right)+\mathcal{O}\left(\Delta \phi^{2}\right) \\
= & \int_{0}^{\hbar \beta} \mathrm{d} \tau^{\prime} \chi\left(\tau, \tau^{\prime}\right) \Delta \phi\left(\tau^{\prime}\right)+\mathcal{O}\left(\Delta \phi^{2}\right) .
\end{aligned}
$$

Here, the susceptibility $\chi\left(\tau, \tau^{\prime}\right)$ has been defined as

$$
\chi\left(\tau, \tau^{\prime}\right) \equiv-\frac{1}{\hbar}\left\langle T_{\tau} \hat{I}_{Q}(\tau) \hat{Q}\left(\tau^{\prime}\right)\right\rangle_{0},
$$

while the differential $\mathcal{D} \psi$ refers to all Grassmann fields $\psi_{\alpha \sigma}$ and $\bar{\psi}_{\alpha \sigma}$, and those contained in the dot spin operator $\tau$. In the above, $\langle\ldots\rangle$ denotes the full expectation value, while $\langle\ldots\rangle_{0}$ refers to the expectation value in absence of a potential gradient; the zeroth order term vanishes because there is no transport if the potential gradient is zero, and the bubble diagrams $\sim\left\langle\hat{I}_{Q}(\tau)\right\rangle_{0}\left\langle\hat{Q}\left(\tau^{\prime}\right)\right\rangle_{0}$ vanish for the same reason. Moreover, the time ordering operator $T_{\tau}$ originates from the slicing procedure used in the derivation of the functional integral formalism. It is now important to note that the susceptibility is an imaginary time Green function,

$$
\chi\left(\tau, \tau^{\prime}\right)=\frac{1}{\hbar} C_{I Q}^{\tau}\left(\tau-\tau^{\prime}\right),
$$


where we used the fact that the bare Hamiltonian (i.e. in absence of $\Delta \phi$ ) is time-independent to justify the statement that any bare two-point function $\left\langle\hat{A}(\tau) \hat{B}\left(\tau^{\prime}\right)\right\rangle_{0}$ can be written as a function only depending on the time difference $\left(\tau-\tau^{\prime}\right)$. Fourier transforming Eq. A.36) to Matsubara frequencies, analytically continuing to real frequencies and inverse Fourier transforming back to time, Eq. A.36 becomes

$$
\left\langle\hat{I}_{Q}\right\rangle(t)=\int_{-\infty}^{\infty} \mathrm{d} t^{\prime} \chi\left(t, t^{\prime}\right) \Delta \phi\left(t^{\prime}\right)+\mathcal{O}\left(\Delta \phi^{2}\right),
$$

with

$$
\chi\left(t, t^{\prime}\right)=\frac{1}{\hbar} C_{I Q}^{\mathrm{R}}\left(t-t^{\prime}\right)=-\frac{i}{\hbar} \theta\left(t-t^{\prime}\right)\left\langle\left[\hat{I}_{Q}(t), \hat{Q}\left(t^{\prime}\right)\right]\right\rangle_{0} .
$$

This gives us an expression for the linear response susceptibility of the current corresponding to charge $Q$ due to a potential drop $\Delta \phi$, in terms of a "bare" expectation value.

The above susceptibility can also be written as a current-current correlation function. To do so, we introduce another potential $A$ such that $\Delta \phi(t)=-\partial_{t} A(t)$. Integrating Eq. A.39 by parts and using the definition of the current from Eq. 4.13), we find

$$
\begin{aligned}
& \left\langle\hat{I}_{Q}\right\rangle(t) \\
& =\int_{-\infty}^{\infty} \mathrm{d} t^{\prime}\left(-\frac{1}{\hbar} C^{\mathrm{R}}\left(t-t^{\prime}\right)+\frac{i}{\hbar} \delta\left(t-t^{\prime}\right)\left\langle\left[\hat{I}_{Q}(t), \hat{Q}(t)\right]\right\rangle_{0}\right) A\left(t^{\prime}\right)+\mathcal{O}\left(\Delta \phi^{2}\right),
\end{aligned}
$$

where we applied the product rule to obtain two terms, and $C^{\mathrm{R}}\left(t-t^{\prime}\right)$ is the current autocorrelator from Eq. (4.57). Note that the boundary terms from integrating by parts vanish due to the procedure of adiabatically switching the potential on and off in the distant past and future (also used in the Keldysh formalism from Sec. 4.2.4), such that $A(t \rightarrow-\infty)=A(t \rightarrow \infty)=0$. Furthermore realizing that the Fourier transform of the equation $\Delta \phi(t)=$ $-\partial_{t} A(t)$ is simply $\Delta \phi(\omega)=i \omega A(\omega)$, we find

$$
\begin{aligned}
\left\langle\hat{I}_{Q}\right\rangle(\omega) & =\left(-\frac{1}{\hbar} C^{\mathrm{R}}(\omega)+\frac{i}{\hbar}\left\langle\left[\hat{I}_{Q}(t), \hat{Q}(t)\right]\right\rangle_{0}\right) A(\omega)+\mathcal{O}\left(\Delta \phi^{2}\right) \\
& =\frac{i}{\hbar \omega}\left(C^{\mathrm{R}}(\omega)-i\left\langle\left[\hat{I}_{Q}(t), \hat{Q}(t)\right]\right\rangle_{0}\right) \Delta \phi(\omega)+\mathcal{O}\left(\Delta \phi^{2}\right),
\end{aligned}
$$


where it should be noted that the term $i\left\langle\left[\hat{I}_{Q}(t), \hat{Q}(t)\right]\right\rangle_{0}$ is a (real) constant, again due to the fact that the bare system is time-independent. Starting from Eq. (4.57) and using the fact that the current operator is defined as minus the time derivative of the charge operator, we can relate this constant to the zero frequency current autocorrelator:

$$
\begin{aligned}
C^{\mathrm{R}}(\omega=0) & =-i \int_{-\infty}^{0} \mathrm{~d} \Delta t\left\langle\left[\hat{I}_{Q}(t), \hat{I}_{Q}(t+\Delta t)\right]\right\rangle_{0} \\
& =i\left\langle\left[\hat{I}_{Q}(t), \hat{Q}(t)\right]\right\rangle_{0}-i\left\langle\left[\hat{I}_{Q}(t), \hat{Q}(-\infty)\right]\right\rangle_{0},
\end{aligned}
$$

the final term being zero. With this, we finally arrive at

$$
\chi(\omega)=\frac{i}{\hbar \omega}\left(C^{\mathrm{R}}(\omega)-C^{\mathrm{R}}(0)\right),
$$

which is the expression used in the main text.

In the dc case, the potential drop is given by $\Delta \phi(\omega)=2 \pi \Delta \phi \delta(\omega)$. As a result, the dc susceptibility is equal to the $\omega \rightarrow 0$ limit of $\chi(\omega)$, as can be seen by inverse Fourier transforming Eq. A.42). In this limit, the above result can be further simplified by noting that $\operatorname{Re}\left[C^{\mathrm{R}}(\omega)\right]=\operatorname{Re}\left[C^{\mathrm{R}}(-\omega)\right]$ and $\operatorname{Im}\left[C^{\mathrm{R}}(\omega)\right]=-\operatorname{Im}\left[C^{\mathrm{R}}(-\omega)\right]\left[79\right.$. The real part of $\left(C^{\mathrm{R}}(\omega)-C^{\mathrm{R}}(0)\right)$ is therefore at least quadratic in $\omega$, such that the dc susceptibility becomes

$$
\chi_{\mathrm{dc}}=\lim _{\omega \rightarrow 0} \frac{1}{\hbar \omega}\left(-\operatorname{Im}\left[C^{\mathrm{R}}(\omega)\right]\right) .
$$

This is the form of the Kubo formula often found in literature.

\section{A.5 Digamma function}

The digamma function is defined as

$$
\Psi(z) \equiv \frac{\mathrm{d} \ln \Gamma(z)}{\mathrm{d} z}=\frac{1}{\Gamma(z)} \frac{\mathrm{d} \Gamma(z)}{\mathrm{d} z},
$$

where $\Gamma(z)$ is the gamma function and $z$ is a complex number. Differentiating the relation $\Gamma(z+1)=z \Gamma(z)$ (where $\operatorname{Re}[z]>0$ ) with respect to $z$, it immediately follows that the digamma function satisfies a somewhat similar relation, namely $\Psi(z+1)=\Psi(z)+1 / z$. Consider now the following sum, 
with $\operatorname{Re}[a]>0$ and $\operatorname{Re}[b]>0$ :

$$
\begin{aligned}
(a-b) \sum_{n=0}^{\infty} \frac{1}{(n+a)(n+b)} & =\sum_{n=0}^{\infty}\left(\frac{1}{n+b}-\frac{1}{n+a}\right) \\
& =\sum_{n=0}^{\infty} \int_{0}^{1} \mathrm{~d} x\left(x^{n+b-1}-x^{n+a-1}\right) \\
& =\int_{0}^{1} \mathrm{~d} x \frac{x^{b-1}-x^{a-1}}{1-x} .
\end{aligned}
$$

To evaluate this integral, we introduce a more general version of this integral,

$$
I(\delta) \equiv \int_{0}^{1} \mathrm{~d} x\left(x^{b-1}-x^{a-1}\right)(1-x)^{\delta-1}=B(b, \delta)-B(a, \delta),
$$

with $\delta>0$, and $B(x, y)$ being the beta function, satisfying the well-known relation $\Gamma(x) \Gamma(y)=B(x, y) \Gamma(x+y)$. The above sum can now be evaluated:

$$
\begin{aligned}
& (a-b) \sum_{n=0}^{\infty} \frac{1}{(n+a)(n+b)} \\
& =\lim _{\delta \rightarrow 0^{+}} I(\delta)=\lim _{\delta \rightarrow 0^{+}}(B(b, \delta)-B(a, \delta)) \\
& =\lim _{\delta \rightarrow 0^{+}} \Gamma(\delta)\left(\frac{\Gamma(b)}{\Gamma(b+\delta)}-\frac{\Gamma(a)}{\Gamma(a+\delta)}\right) \\
& =\lim _{\delta \rightarrow 0^{+}} \Gamma(\delta+1)\left(\frac{\Gamma(b)-\Gamma(b+\delta)}{\delta} \frac{1}{\Gamma(b+\delta)}-\frac{\Gamma(a)-\Gamma(a+\delta)}{\delta} \frac{1}{\Gamma(a+\delta)}\right) \\
& =\Psi(a)-\Psi(b) .
\end{aligned}
$$

In addition, we can recursively apply the relation $\Psi(a+1)=\Psi(a)+1 / a$ to find another sum:

$$
\begin{aligned}
& \Psi(a+n)=\Psi(a+n-1)+\frac{1}{a+n-1} \\
& =\Psi(a+n-2)+\frac{1}{a+n-2}+\frac{1}{a+n-1} \\
& =\Psi(a)+\sum_{n^{\prime}=0}^{n-1} \frac{1}{n^{\prime}+a},
\end{aligned}
$$


where $n \in \mathbb{N}_{>0}$ and $\operatorname{Re}[a]>0$. Using this, we can evaluate the following sum as well:

$$
\begin{aligned}
\sum_{n^{\prime}=0}^{n-1} & \frac{1}{n^{\prime}+a} \frac{1}{n^{\prime}-b-n+1} \\
& =-\frac{1}{a+b+n-1}\left(\sum_{n^{\prime}=0}^{n-1} \frac{1}{n^{\prime}+a}-\sum_{n^{\prime}=0}^{n-1} \frac{1}{n^{\prime}-b-n+1}\right) \\
& =-\frac{1}{a+b+n-1}\left(\sum_{n^{\prime}=0}^{n-1} \frac{1}{n^{\prime}+a}+\sum_{n^{\prime}=0}^{n-1} \frac{1}{n^{\prime}+b}\right) \\
& =\frac{1}{a+b+n-1}(\Psi(a)-\Psi(a+n)+\Psi(b)-\Psi(b+n)),
\end{aligned}
$$

where the second term of the third line involves a redefinition according to $n^{\prime} \rightarrow-n^{\prime}+n-1$. Finally, we use Eq. A.50 to evaluate one final sum:

$$
\begin{aligned}
\sum_{n^{\prime}=0}^{n-1} \frac{1}{\left(n^{\prime}+a\right)^{2}} & =\lim _{\delta \rightarrow 0^{+}} \sum_{n^{\prime}=0}^{n-1} \frac{1}{\left(n^{\prime}+a\right)\left(n^{\prime}+a+\delta\right)} \\
& =\lim _{\delta \rightarrow 0^{+}}-\frac{1}{\delta} \sum_{n^{\prime}=0}^{n-1}\left(\frac{1}{n^{\prime}+a+\delta}-\frac{1}{n^{\prime}+a}\right) \\
& =\lim _{\delta \rightarrow 0^{+}}-\frac{1}{\delta}(\Psi(a+n+\delta)-\Psi(a+\delta)-\Psi(a+n)+\Psi(a)) \\
& =\psi^{(1)}(a)-\psi^{(1)}(a+n),
\end{aligned}
$$

where $\psi^{(1)}(z)$ is the trigamma function (i.e. the derivative of the digamma function with respect to $z$ ).

Let us also briefly consider two specific expansions of the trigamma function. Using the known values of the gamma function and its derivatives at $z=1 / 2$, the first one is simple:

$$
x \psi^{(1)}\left(\frac{1}{2}+x\right)=x \psi^{(1)}\left(\frac{1}{2}\right)+\mathcal{O}\left(x^{2}\right)=\frac{\pi^{2} x}{2}+\mathcal{O}\left(x^{2}\right) .
$$

The second one is more complicated, and requires the asymptotic series

$$
\Psi(x) \sim \ln x-\frac{1}{2 x}-\frac{1}{12 x^{2}}+\mathcal{O}\left((1 / x)^{4}\right),
$$

valid for real variable $x \gg 1$, which can be derived from the Stirling series. Taking the derivative of this asymptotic series and plugging in the argument 
we are interested in:

$$
\frac{1}{x} \psi^{(1)}\left(\frac{1}{2}+\frac{1}{x}\right)=\frac{1}{x}\left(\frac{x}{1+x / 2}+\frac{x^{2}}{2(1+x / 2)^{2}}+\frac{x^{3}}{6(1+x / 2)^{3}}\right)+\mathcal{O}\left(x^{4}\right) .
$$

Also applying the binomial series,

$$
(1+x)^{n}=\sum_{k=0}^{\infty} \frac{n !}{(n-k) ! k !} x^{k},
$$

it is straightforward to show that Eq. A.55) reduces to

$$
\frac{1}{x} \psi^{(1)}\left(\frac{1}{2}+\frac{1}{x}\right)=1-\frac{x^{2}}{12}+\mathcal{O}\left(x^{4}\right) .
$$

\section{A.6 Bessel functions of the first kind}

For integers $n$, the Bessel functions of the first kind are defined as

$$
J_{n}(\alpha) \equiv \sum_{m=0}^{\infty} \frac{(-1)^{m}}{m !(m+n) !}\left(\frac{\alpha}{2}\right)^{2 m+n} .
$$

Using this expression, the following sum can be evaluated:

$$
\begin{aligned}
\sum_{n=-\infty}^{\infty} e^{-i n \omega t} J_{n}(\alpha) & =\sum_{n=-\infty}^{\infty} e^{-i n \omega t} \sum_{m=0}^{\infty} \frac{(-1)^{m}}{m !(m+n) !}\left(\frac{\alpha}{2}\right)^{2 m+n} \\
& =\sum_{m=0}^{\infty} \frac{1}{m !}\left(-\frac{\alpha}{2}\right)^{m} \sum_{n=-\infty}^{\infty} \frac{1}{(m+n) !}\left(\frac{\alpha}{2}\right)^{m+n} e^{-i n \omega t} \\
& =\sum_{m=0}^{\infty} \frac{1}{m !}\left(-\frac{\alpha}{2} e^{i \omega t}\right)^{m} \sum_{n=-\infty}^{\infty} \frac{1}{(m+n) !}\left(\frac{\alpha}{2} e^{-i \omega t}\right)^{m+n} \\
& =\sum_{m=0}^{\infty} \frac{1}{m !}\left(-\frac{\alpha}{2} e^{i \omega t}\right)^{m} \sum_{n^{\prime}=0}^{\infty} \frac{1}{n^{\prime} !}\left(\frac{\alpha}{2} e^{-i \omega t}\right)^{n^{\prime}} \\
& =e^{-\frac{\alpha}{2}\left(e^{i \omega t}-e^{-i \omega t}\right)} \\
& =e^{-i \alpha \sin (\omega t)}
\end{aligned}
$$

where in the fourth line we used the fact that the terms corresponding to $m+n<0$ vanish (since $1 / n !=0$ if $n$ is a negative integer), and defining a 
new dummy index $n^{\prime}$ by using the observation that the second infinite sum must be independent of $m$. From the definition of the Bessel functions, it also follows that they can be expanded as

$$
J_{0}(\alpha)=1+\mathcal{O}\left(\alpha^{2}\right), \quad J_{n \neq 0}(\alpha)=\frac{(\operatorname{sgn}(n))^{|n|}}{|n| !}\left(\frac{\alpha}{2}\right)^{|n|}+\mathcal{O}\left(\alpha^{|n|+2}\right) .
$$

As such, the expansion of a general $J_{n}(\alpha)$ is given by

$$
J_{n}(\alpha)=\delta_{n, 0}+\frac{\alpha}{2}\left(\delta_{n, 1}-\delta_{n,-1}\right)+\mathcal{O}\left(\alpha^{2}\right) .
$$

\section{A.7 Ac current in absence of a magnetic field}

In this section, we will evaluate the remaining integral from Eq. (5.39) for $B=0$. We have two integrals to consider:

$$
\Gamma \int_{-\infty}^{\infty} \mathrm{d} \epsilon \frac{n_{F}(\epsilon)}{\epsilon^{2}+\Gamma^{2}}, \quad \int_{-\infty}^{\infty} \mathrm{d} \epsilon\left(-\frac{n_{F}\left(\epsilon-\epsilon_{0}\right)}{\epsilon-i \Gamma}+\frac{n_{F}\left(\epsilon-\epsilon_{0}-n \hbar \omega_{0}\right)}{\epsilon+i \Gamma}\right),
$$

where $\epsilon_{0} \equiv e V_{0} / 2+n^{\prime} \hbar \omega_{0}$. To evaluate these integrals, we again use the Matsubara representation of the Fermi-Dirac distribution from Eq. (5.16). Employing the same techniques as for the dc case in Sec. 5.2, the first integral can be written as

$$
\begin{aligned}
\Gamma \int_{-\infty}^{\infty} \mathrm{d} \epsilon \frac{n_{F}(\epsilon)}{\epsilon^{2}+\Gamma^{2}} & =\frac{2}{\beta} \sum_{j=0}^{\infty} \operatorname{Re}\left[\int_{-\infty}^{\infty} \mathrm{d} \epsilon \frac{\Gamma}{\epsilon^{2}+\Gamma^{2}} \frac{e^{i \omega_{j} 0^{+}}}{i \hbar \omega_{j}-\epsilon}\right] \\
& =\frac{2 \pi}{\beta} \sum_{j=0}^{\infty} \operatorname{Re}\left[\frac{e^{i \omega_{j} 0^{+}}}{i \hbar \omega_{j}+i \Gamma}\right] .
\end{aligned}
$$

The second integral can be evaluated in a similar fashion:

$$
\begin{aligned}
\int_{-\infty}^{\infty} \mathrm{d} \epsilon & \left(-\frac{n_{F}(\epsilon)}{\epsilon+\epsilon_{0}-i \Gamma}+\frac{n_{F}(\epsilon)}{\epsilon+\epsilon_{0}+n \hbar \omega_{0}+i \Gamma}\right) \\
= & -\frac{n \hbar \omega_{0}+2 i \Gamma}{\beta} \int_{-\infty}^{\infty} \mathrm{d} \epsilon \sum_{j=0}^{\infty} \frac{1}{\left(\epsilon+\epsilon_{0}-i \Gamma\right)\left(\epsilon+\epsilon_{0}+n \hbar \omega_{0}+i \Gamma\right)} \\
& \times\left(\frac{e^{i \omega_{j} 0^{+}}}{i \hbar \omega_{j}-\epsilon}+\frac{e^{-i \omega_{j} 0^{+}}}{-i \hbar \omega_{j}-\epsilon}\right)
\end{aligned}
$$




$$
\begin{aligned}
= & \frac{2 \pi}{\beta} \sum_{j=0}^{\infty}\left(\frac{1}{\hbar \omega_{j}+\Gamma+i \epsilon_{0}}-\frac{1}{\hbar \omega_{j}+\Gamma-i\left(\epsilon_{0}+n \hbar \omega_{0}\right)}\right) \\
& +\frac{2 \pi}{\beta} \sum_{j=0}^{\infty}\left(\frac{e^{-i \omega_{j} 0^{+}}-1}{\hbar \omega_{j}+\Gamma+i \epsilon_{0}}-\frac{e^{i \omega_{j} 0^{+}}-1}{\hbar \omega_{j}+\Gamma-i\left(\epsilon_{0}+n \hbar \omega_{0}\right)}\right),
\end{aligned}
$$

where we wrote $e^{ \pm i \omega_{j} 0^{+}} \rightarrow 1+\left(e^{ \pm i \omega_{j} 0^{+}}-1\right)$ for reasons that will become clear in a moment. For the final line, we can use the fact that it vanishes unless $j \rightarrow \infty$ to replace the summand with its $j \rightarrow \infty$ value. Doing so, we can recognize this second term to be equal to $-2 i$ times the integral from Eq. A.63). Looking at Eq. (5.39), we see that the two cancel each other, and so we only need to evaluate the first term of Eq. A.64):

$$
\begin{aligned}
\frac{2 \pi}{\beta} \sum_{j=0}^{\infty} & \left(\frac{1}{\hbar \omega_{j}+\Gamma+i \epsilon_{0}}-\frac{1}{\hbar \omega_{j}+\Gamma-i\left(\epsilon_{0}+n \hbar \omega_{0}\right)}\right) \\
& =-\frac{i \beta}{2 \pi} \sum_{j=0}^{\infty} \frac{2 \epsilon_{0}+n \hbar \omega_{0}}{\left(j+\frac{1}{2}+\frac{\beta\left(\Gamma+i \epsilon_{0}\right)}{2 \pi}\right)\left(j+\frac{1}{2}+\frac{\beta\left(\Gamma-i\left(\epsilon_{0}+n \hbar \omega_{0}\right)\right)}{2 \pi}\right)} \\
& =-\Psi\left(\frac{1}{2}+\frac{\Gamma+i \epsilon_{0}}{2 \pi k_{B} T}\right)+\Psi\left(\frac{1}{2}+\frac{\Gamma-i\left(\epsilon_{0}+n \hbar \omega_{0}\right)}{2 \pi k_{B} T}\right) .
\end{aligned}
$$

Plugging this back into the expression for $I_{n}$, we obtain

$$
\begin{aligned}
I_{n}= & -\frac{i e \Gamma}{4 \pi \hbar} \sum_{n^{\prime}=-\infty}^{\infty} J_{n^{\prime}}\left(\frac{e \Delta V}{2 \hbar \omega_{0}}\right) J_{n^{\prime}+n}\left(\frac{e \Delta V}{2 \hbar \omega_{0}}\right)\left(\Psi\left(\frac{1}{2}+\frac{\Gamma+i\left(e V_{0} / 2+n^{\prime} \hbar \omega_{0}\right)}{2 \pi k_{B} T}\right)\right. \\
& \left.-\Psi\left(\frac{1}{2}+\frac{\Gamma-i\left(e V_{0} / 2+\left(n^{\prime}+n\right) \hbar \omega_{0}\right)}{2 \pi k_{B} T}\right)\right) .
\end{aligned}
$$

Note that the Bessel functions reduce to $J_{n}(0)=\delta_{n, 0}$ in the limit $\Delta V \rightarrow 0$. As a result, only $I_{0}$ remains non-zero, such that $\omega_{0}$ completely vanishes from the expression and we indeed recover the correct dc limit.

\section{A.8 Vanishing bubble diagrams}

In the first part of Sec. 6.2, we claimed that the various disconnected bubble diagrams that appear in the heat current autocorrelator are all equal to zero. In the following, we will back up this claim. First, let us consider the bubble diagram $\sum_{k, k^{\prime}}\left\langle\psi_{k}^{\dagger}(\tau) \psi_{k^{\prime}}(\tau)\right\rangle_{0}$, where $\psi_{k}$ is a fermionic field operator that 
only appears in the kinetic term of the Hamiltonian. In terms of Matsubara frequencies and disregarding the now redundant Nambu basis, the Green function corresponding to this field is given by

$$
G_{k k^{\prime}}\left(i \omega_{n}\right)=\delta_{k, k^{\prime}} \frac{\hbar}{i \hbar \omega_{n}-\epsilon_{k}} .
$$

In order to make sense of the bubble diagram, it is necessary to implement point-splitting. The only physical way (i.e. without breaking causality) to achieve this is to first annihilate a fundamental particle, then create one in the new state. For a fundamental field $c$, the point-splitting procedure we adopt is therefore $c^{\dagger}(\tau) c(\tau) \rightarrow c^{\dagger}\left(\tau^{+}\right) c(\tau)$, with $\tau^{+} \equiv \tau+0^{+}$. At this point, it is crucial to recall that the field $\psi_{k}$ is not fundamental, but instead describes excitations about the ground state.$^{2}$ For fermions, this is the state in which all states up to the Fermi energy are filled, and all states above the Fermi energy are empty. Given that the momentum $k$ is measured with respect to the Fermi momentum $k_{F}$, the excitation field $\psi_{k}$ is thus defined as

$$
\psi_{k}(\tau) \equiv \begin{cases}c_{k}(\tau), & k>0, \\ c_{k}^{\dagger}(\tau), & k \leq 0,\end{cases}
$$

where $c_{k}$ is the field corresponding to the fundamental particles $3^{3}$ Using this, the causal point-splitting procedure for the excitation field becomes

$$
\begin{aligned}
\psi_{k}^{\dagger}(\tau) \psi_{k}(\tau) & \rightarrow \theta(k) c_{k}^{\dagger}\left(\tau^{+}\right) c_{k}(\tau)+\theta(-k) c_{k}(\tau) c_{k}^{\dagger}\left(\tau^{+}\right) \\
& =\theta(k) \psi_{k}^{\dagger}\left(\tau^{+}\right) \psi_{k}(\tau)-\theta(-k) \psi_{k}\left(\tau^{+}\right) \psi_{k}^{\dagger}(\tau) .
\end{aligned}
$$

We apply this point-splitting procedure to the required expectation value:

$$
\begin{aligned}
\left\langle\psi_{k}^{\dagger}(\tau) \psi_{k}(\tau)\right\rangle_{0} & \rightarrow \theta(k)\left\langle\psi_{k}^{\dagger}\left(\tau^{+}\right) \psi_{k}(\tau)\right\rangle_{0}-\theta(-k)\left\langle\psi_{k}\left(\tau^{+}\right) \psi_{k}^{\dagger}(\tau)\right\rangle_{0} \\
& =\theta(k) G_{k k}\left(-0^{+}\right)+\theta(-k) G_{k k}\left(0^{+}\right) \\
& =\frac{\theta(k)}{\hbar \beta} \sum_{n=-\infty}^{\infty} \frac{\hbar}{i \hbar \omega_{n}-\epsilon_{k}} e^{i \omega_{n} 0^{+}}-\frac{\theta(-k)}{\hbar \beta} \sum_{n=-\infty}^{\infty} \frac{\hbar}{i \hbar \omega_{n}+\epsilon_{k}} e^{i \omega_{n} 0^{+}} \\
& =\theta(k) n_{F}\left(\epsilon_{k}\right)-\theta(-k) n_{F}\left(-\epsilon_{k}\right),
\end{aligned}
$$

\footnotetext{
${ }^{2}$ In Appendix A.1 it was not necessary to take this subtlety into account. The reason for this is that we only used the point-splitting as a mathematical tool in the previous appendix, whereas we now use it for the calculation of observable quantities.

${ }^{3}$ This particular example is about right-movers. Left-movers can be considered using the same methods, leading to the same conclusions.
} 
where we used Eq. (5.16) to introduce the Fermi-Dirac distribution. In the continuum limit, the desired bubble diagram can now be written as

$$
\begin{aligned}
\sum_{k, k^{\prime}}\left\langle\psi_{k}^{\dagger}(\tau) \psi_{k^{\prime}}(\tau)\right\rangle_{0} & =\frac{L}{2 \pi}\left(\int_{0}^{\infty} \mathrm{d} k n_{F}\left(\epsilon_{k}\right)-\int_{-\infty}^{0} \mathrm{~d} k n_{F}\left(-\epsilon_{k}\right)\right) \\
& =\frac{L}{2 \pi} \int_{0}^{\infty} \mathrm{d} k\left(n_{F}\left(\epsilon_{k}\right)-n_{F}\left(-\epsilon_{-k}\right)\right) .
\end{aligned}
$$

In the present case, there is particle-hole symmetry (i.e. $\epsilon_{k}=-\epsilon_{-k}$ ), such that this bubble diagram trivially vanishes.

In addition, we prove that the combination $\left(C_{35}+C_{53}\right)$ is indeed equal to zero in absence of a magnetic field. The underlying reason is that each term of this combination contains a bubble diagram as well. Using that the $a$ Majorana fermion is decoupled from the rest of the system (due to $B=0$ ), it can be expressed as

$$
\begin{aligned}
C_{35}(\tau)+C_{53}(\tau)= & -\frac{i \pi v_{F} \Lambda g_{\perp}}{2^{5 / 2} \hbar L^{3 / 2}} \sum_{k, k^{\prime}, k^{\prime \prime}}\left(\epsilon_{k^{\prime}}-\epsilon_{k}\right)\langle a(\tau) a(0)\rangle_{0} \\
& \times\left(\left\langle\left(\psi_{s f, k^{\prime \prime}}^{\dagger}(\tau)+\psi_{s f, k^{\prime \prime}}(\tau)\right) \psi_{s f, k}^{\dagger}(0) \psi_{s f, k^{\prime}}(0) b(0)\right\rangle_{0}\right. \\
& \left.+\left\langle\psi_{s f, k}^{\dagger}(\tau) \psi_{s f, k^{\prime}}(\tau) b(\tau)\left(\psi_{s f, k^{\prime \prime}}^{\dagger}(0)+\psi_{s f, k^{\prime \prime}}(0)\right)\right\rangle_{0}\right) .
\end{aligned}
$$

The expectation values inside the brackets can be evaluated using Wick's theorem. Before doing so, we note that the diagonal components of the propagator $\mathbf{L}_{k k^{\prime}}$ remain the same upon interchanging $k$ and $k^{\prime}$, which can straightforwardly be shown by working out the matrix multiplications in the Green functions from Sec.4.2.3. Multiplied with $\left(\epsilon_{k^{\prime}}-\epsilon_{k}\right)$ and summed over $k$ and $k^{\prime}$, this cancels all terms proportional to $\left\langle\psi_{s f, k}^{\dagger}(\tau) \psi_{s f, k^{\prime}}(\tau)\right\rangle_{0}$. Moreover, the propagator $\mathbf{G}_{l d, k}\left(i \omega_{n}\right)$ is proportional to $\omega_{n}^{-2}$ as $n \rightarrow \pm \infty$. As a result, this Green function is independent of the point-splitting procedure: it can simply be written as $\mathbf{G}_{l d, k}(0)=(\hbar \beta)^{-1} \sum_{n} \mathbf{G}_{l d, k}\left(i \omega_{n}\right)$. Again working out the matrix multiplications, and using that $D_{b b}\left(i \omega_{n}\right)$ is odd in $\omega_{n}$ to remove the odd part of the summand, we find

$$
\left\langle\psi_{s f, k}(\tau) b(\tau)\right\rangle_{0}=\left\langle\psi_{s f, k}^{\dagger}(\tau) b(\tau)\right\rangle_{0}=-\frac{\sqrt{2} g_{\perp}}{\sqrt{L}} \frac{1}{\hbar \beta} \sum_{n=-\infty}^{\infty} \frac{\hbar \omega_{n}}{\left(\hbar \omega_{n}\right)^{2}+\epsilon_{k}^{2}} D_{b b}\left(i \omega_{n}\right) .
$$


With the above in mind, we now apply Wick's theorem. After a series of simplifications, this gives

$$
\begin{aligned}
C_{35}(\tau)+C_{53}(\tau)= & \frac{i \pi v_{F} \Lambda g_{\perp}^{2}}{2 \hbar L^{2}} \frac{1}{\hbar \beta} \sum_{k, k^{\prime}, k^{\prime \prime}}\left(\epsilon_{k^{\prime}}-\epsilon_{k}\right) D_{a a}(\tau) \\
& \times \sum_{n=-\infty}^{\infty} \frac{\hbar \omega_{n}}{\left(\hbar \omega_{n}\right)^{2}+\epsilon_{k^{\prime}}^{2}} D_{b b}\left(i \omega_{n}\right) \sum_{\mu \nu} G_{l l, k k^{\prime \prime}, \mu \nu}(\tau) .
\end{aligned}
$$

It is straightforward to check that the second line is even in all momenta, such that all terms of the summand are odd in either $k$ or $k^{\prime}$. This immediately leads to the conclusion that the combination $\left(C_{35}+C_{53}\right)$ is equal to zero.

\section{A.9 Heat transport: supplementary material}

Below, we elaborate on some of the steps from Sec. 6.2, still taking $n>0$ in every calculation.

- $\boldsymbol{C}_{\mathbf{1 1}}$ : the sum $\sum_{n^{\prime}=0}^{n-1} \frac{\frac{1}{2}-2\left(n^{\prime}-n+\frac{1}{2}\right)^{2}}{n^{\prime}+\frac{1}{2}+\frac{\beta \Gamma}{2 \pi}}$

In order to evaluate this object, we first refer back to Eq. A.50 and use it to calculate two related sums:

$$
\begin{aligned}
\sum_{n^{\prime}=0}^{n-1} \frac{n^{\prime}}{n^{\prime}+a} & =\sum_{n^{\prime}=0}^{n-1}\left(1-\frac{a}{n^{\prime}+a}\right) \\
& =n-a(\Psi(a+n)-\Psi(a)), \\
\sum_{n^{\prime}=0}^{n-1} \frac{n^{\prime 2}}{n^{\prime}+a} & =\sum_{n^{\prime}=0}^{n-1}\left(n^{\prime}-a+\frac{a^{2}}{n^{\prime}+a}\right) \\
& =\frac{n(n-2 a-1)}{2}+a^{2}(\Psi(a+n)-\Psi(a)),
\end{aligned}
$$

where $n>0$ and $\operatorname{Re}[a]>0$. Using the above, a straightforward calculation leads to

$$
\begin{aligned}
\sum_{n^{\prime}=0}^{n-1} \frac{\frac{1}{2}-2\left(n^{\prime}-n+\frac{1}{2}\right)^{2}}{n^{\prime}+\frac{1}{2}+\frac{\beta \Gamma}{2 \pi}}= & -2 \sum_{n^{\prime}=0}^{n-1} \frac{n^{\prime 2}}{n^{\prime}+\frac{1}{2}+\frac{\beta \Gamma}{2 \pi}}+2(2 n-1) \sum_{n^{\prime}=0}^{n-1} \frac{n^{\prime}}{n^{\prime}+\frac{1}{2}+\frac{\beta \Gamma}{2 \pi}} \\
& -2 n(n-1) \sum_{n^{\prime}=0}^{n-1} \frac{1}{n^{\prime}+\frac{1}{2}+\frac{\beta \Gamma}{2 \pi}}
\end{aligned}
$$




$$
\begin{aligned}
= & 3 n^{2}+\frac{\beta \Gamma n}{\pi} \\
& +\left(\frac{1}{2}-2\left(n+\frac{\beta \Gamma}{2 \pi}\right)^{2}\right)\left(\Psi\left(\frac{1}{2}+\frac{\beta \Gamma}{2 \pi}+n\right)-\Psi\left(\frac{1}{2}+\frac{\beta \Gamma}{2 \pi}\right)\right) .
\end{aligned}
$$

Expanded to linear order in $n$, this can be written as

$$
\sum_{n^{\prime}=0}^{n-1} \frac{\frac{1}{2}-2\left(n^{\prime}-n+\frac{1}{2}\right)^{2}}{n^{\prime}+\frac{1}{2}+\frac{\beta \Gamma}{2 \pi}}=\left[\frac{\beta \Gamma}{\pi}+\left(\frac{1}{2}-\frac{\beta^{2} \Gamma^{2}}{2 \pi^{2}}\right) \psi^{(1)}\left(\frac{1}{2}+\frac{\beta \Gamma}{2 \pi}\right)\right] n+\mathcal{O}\left(n^{2}\right) .
$$

\section{- $C_{22}+C_{44}+C_{24}+C_{42}$ : expanding in $n$}

As was discussed in Sec. 6.2 , the combination $\left(C_{22}+C_{44}+C_{24}+C_{42}\right)$ cannot be calculated exactly, such that we have to expand Eq. (6.48) in $n$ before evaluating the integral over $\epsilon$. First, the sum over the terms involving $\omega_{n^{\prime}-n}$ has to be split into two parts. This is necessary due to the fact that the conditions $\operatorname{Re}[a]>0, \operatorname{Re}[b]>0$ of Eqs. (A.49) and (A.51) are not satisfied in the initial expression. Doing so, we find

$$
\begin{aligned}
\sum_{n^{\prime}=0}^{\infty}\left(\frac{\hbar \omega_{n^{\prime}+n}+\Gamma}{\left(\hbar \omega_{n^{\prime}+n}\right)^{2}+(2 \epsilon)^{2}}+\frac{\hbar \omega_{n^{\prime}-n}+\Gamma}{\left(\hbar \omega_{n^{\prime}-n}\right)^{2}+(2 \epsilon)^{2}}\right) \frac{1}{\hbar \omega_{n^{\prime}}+\Gamma} \\
=\left(\frac{\beta}{2 \pi}\right)^{2}\left(\sum_{n^{\prime}=0}^{\infty} \frac{n^{\prime}+n+\frac{1}{2}+\frac{\beta \Gamma}{2 \pi}}{\left(n^{\prime}+n+\frac{1}{2}\right)^{2}+\left(\frac{\beta \epsilon}{\pi}\right)^{2}} \frac{1}{n^{\prime}+\frac{1}{2}+\frac{\beta \Gamma}{2 \pi}}\right. \\
\quad+\sum_{n^{\prime}=0}^{\infty} \frac{n^{\prime}+\frac{1}{2}+\frac{\beta \Gamma}{2 \pi}}{\left(n^{\prime}+\frac{1}{2}\right)^{2}+\left(\frac{\beta \epsilon}{\pi}\right)^{2}} \frac{1}{n^{\prime}+n+\frac{1}{2}+\frac{\beta \Gamma}{2 \pi}} \\
\left.\quad+\sum_{n^{\prime}=0}^{n-1} \frac{n^{\prime}+\frac{1}{2}-\frac{\beta \Gamma}{2 \pi}}{\left(n^{\prime}+\frac{1}{2}\right)^{2}+\left(\frac{\beta \epsilon}{\pi}\right)^{2}} \frac{1}{n^{\prime}-n+\frac{1}{2}-\frac{\beta \Gamma}{2 \pi}}\right),
\end{aligned}
$$

where we have written $n^{\prime} \rightarrow n^{\prime}+n$ in the second sum on the right-hand side, and $n^{\prime} \rightarrow-n^{\prime}+n-1$ in the third sum. Furthermore rewriting all of the sums by using

$$
\begin{aligned}
& \frac{x}{x^{2}+y^{2}}=\frac{1}{2}\left(\frac{1}{x-i y}+\frac{1}{x+i y}\right), \\
& \frac{1}{x^{2}+y^{2}}=\frac{1}{2 i y}\left(\frac{1}{x-i y}-\frac{1}{x+i y}\right),
\end{aligned}
$$


every sum is now of the form of one of the sums from Appendix A.5.

$$
\begin{aligned}
& \sum_{n^{\prime}=0}^{\infty}\left(\frac{\hbar \omega_{n^{\prime}+n}+\Gamma}{\left(\hbar \omega_{n^{\prime}+n}\right)^{2}+(2 \epsilon)^{2}}+\frac{\hbar \omega_{n^{\prime}-n}+\Gamma}{\left(\hbar \omega_{n^{\prime}-n}\right)^{2}+(2 \epsilon)^{2}}\right) \frac{1}{\hbar \omega_{n^{\prime}}+\Gamma} \\
& =\frac{\beta^{2}}{8 \pi^{2}}\left(1-\frac{i \Gamma}{2 \epsilon}\right)\left(\sum_{n^{\prime}=0}^{\infty} \frac{1}{n^{\prime}+n+\frac{1}{2}-\frac{i \beta \epsilon}{\pi}} \frac{1}{n^{\prime}+\frac{1}{2}+\frac{\beta \Gamma}{2 \pi}}\right. \\
& \quad+\sum_{n^{\prime}=0}^{\infty} \frac{1}{n^{\prime}+\frac{1}{2}-\frac{i \beta \epsilon}{\pi}} \frac{1}{n^{\prime}+n+\frac{1}{2}+\frac{\beta \Gamma}{2 \pi}} \\
& \left.\quad+\sum_{n^{\prime}=0}^{n-1} \frac{1}{n^{\prime}+\frac{1}{2}+\frac{i \beta \epsilon}{\pi}} \frac{1}{n^{\prime}-n+\frac{1}{2}-\frac{\beta \Gamma}{2 \pi}}\right)+ \text { c.c. }
\end{aligned}
$$

Evaluating the sums and expanding the result in $n$, one finds that there is no linear term, despite the fact that every other power (both even and odd) does appear in the expansion.

\section{- $C_{\mathbf{3}}$ : the $\operatorname{sum} \sum_{n^{\prime}=-\infty}^{\infty} \frac{1}{n^{\prime}-n+\frac{1}{2}} \frac{n^{\prime}+\frac{1}{2}}{\left|n^{\prime}+\frac{1}{2}\right|+\frac{\beta \Gamma}{2 \pi}}$}

As usual, the first step is to split the sum into three parts:

$$
\begin{aligned}
\sum_{n^{\prime}=-\infty}^{\infty} \frac{1}{n^{\prime}-n+\frac{1}{2}} \frac{n^{\prime}+\frac{1}{2}}{\left|n^{\prime}+\frac{1}{2}\right|+\frac{\beta \Gamma}{2 \pi}}= & \sum_{n^{\prime}=0}^{\infty} \frac{1}{n^{\prime}+n+\frac{1}{2}} \frac{n^{\prime}+\frac{1}{2}}{n^{\prime}+\frac{1}{2}+\frac{\beta \Gamma}{2 \pi}} \\
& +\sum_{n^{\prime}=0}^{\infty} \frac{1}{n^{\prime}+\frac{1}{2}} \frac{n^{\prime}+n+\frac{1}{2}}{n^{\prime}+n+\frac{1}{2}+\frac{\beta \Gamma}{2 \pi}} \\
& -\sum_{n^{\prime}=0}^{n-1} \frac{1}{n^{\prime}+\frac{1}{2}} \frac{n^{\prime}-n+\frac{1}{2}}{n^{\prime}-n+\frac{1}{2}-\frac{\beta \Gamma}{2 \pi}} .
\end{aligned}
$$

Here, we have again written $n^{\prime} \rightarrow-n^{\prime}-1$ in the first part, $n^{\prime} \rightarrow n^{\prime}+n$ in the second part, and $n^{\prime} \rightarrow-n^{\prime}+n-1$ in the third part. Each of these sums is subsequently split into two more sums, e.g.

$$
\begin{aligned}
\sum_{n^{\prime}=0}^{\infty} \frac{1}{n^{\prime}+n+\frac{1}{2}} & \frac{n^{\prime}+\frac{1}{2}}{n^{\prime}+\frac{1}{2}+\frac{\beta \Gamma}{2 \pi}} \\
& =\sum_{n^{\prime}=0}^{\infty} \frac{1}{n^{\prime}+n+\frac{1}{2}}-\frac{\beta \Gamma}{2 \pi} \sum_{n^{\prime}=0}^{\infty} \frac{1}{n^{\prime}+n+\frac{1}{2}} \frac{1}{n^{\prime}+\frac{1}{2}+\frac{\beta \Gamma}{2 \pi}} .
\end{aligned}
$$

Two of the six resulting sums diverge, and should be understood to have a finite cut-off $N \sim \beta \Lambda$ (see footnote 1 of Chapter 6). Implementing this cutoff, all of the six sums are now of the form of one of those discussed in 
Appendix A.5, such that we find

$$
\begin{aligned}
\sum_{n^{\prime}=-N}^{N-1} \frac{1}{n^{\prime}-n+\frac{1}{2}} \frac{n^{\prime}+\frac{1}{2}}{\left|n^{\prime}+\frac{1}{2}\right|+\frac{\beta \Gamma}{2 \pi}} \\
=\Psi\left(\frac{1}{2}-n+N\right)+\Psi\left(\frac{1}{2}+n+N\right) \\
\quad-\frac{2}{n^{2}-\left(\frac{\beta \Gamma}{2 \pi}\right)^{2}}\left(n^{2} \Psi\left(\frac{1}{2}+n\right)-\left(\frac{\beta \Gamma}{2 \pi}\right)^{2} \Psi\left(\frac{1}{2}+\frac{\beta \Gamma}{2 \pi}\right)\right),
\end{aligned}
$$

where we have taken $N \rightarrow \infty$ whenever possible. Again, despite the fact that this expression is not even in $n$, a Taylor expansion in $n$ reveals that it does not contain a linear term.

\section{- $C_{55}$ : analytic expression}

Five of the terms appearing in $C_{55}$ are bubble diagrams proportional to

$$
\sum_{k, k^{\prime}}\left(\epsilon_{k^{\prime}}-\epsilon_{k}\right)\left\langle\psi_{s f, k}^{\dagger}(\tau) \psi_{s f, k^{\prime}}(\tau)\right\rangle_{0} .
$$

As was discussed in Appendix A.8, the expectation value appearing in this expression is invariant under $k \leftrightarrow k^{\prime}$, such that this entire bubble diagram vanishes after summing over the momenta. In addition to these vanishing bubble diagrams, there are four terms involving the bubble diagrams from Eq. A.73). Carefully combining these terms, relabelling the momenta whenever necessary and discarding the terms that are odd in any of the momenta, they are given by

$$
\begin{aligned}
& \frac{\left(\pi v_{F} g_{\perp}\right)^{2}}{2 L^{3}} \frac{1}{(\hbar \beta)^{2}} \sum_{k, k^{\prime}, k^{\prime \prime}} \epsilon_{k}^{2} D_{a a}(\tau) \\
& \quad \times \sum_{\mu \nu} L_{0, k, \mu \nu}(\tau) \sum_{n, n^{\prime}} \frac{\hbar \omega_{n}}{\left(\hbar \omega_{n}\right)^{2}+\epsilon_{k^{\prime}}^{2}} D_{b b}\left(i \omega_{n}\right) \frac{\hbar \omega_{n^{\prime}}}{\left(\hbar \omega_{n^{\prime}}\right)^{2}+\epsilon_{k^{\prime \prime}}^{2}} D_{b b}\left(i \omega_{n^{\prime}}\right),
\end{aligned}
$$

where $L_{0, k, \mu \nu}$ is the $\mu \nu$ component of the spin-flavour propagator $\mathbf{L}_{0, k}$ in absence of tunnelling. In terms of Matsubara frequencies, the object we have to calculate is thus given by

$$
\begin{aligned}
\sum_{k} \epsilon_{k}^{2} \sum_{n^{\prime}=-\infty}^{\infty} D_{a a}\left(i \omega_{n^{\prime}}\right) \sum_{\mu \nu} & L_{0, k, \mu \nu}\left(-i \omega_{n^{\prime}-n}\right) \\
& \propto \sum_{k} \epsilon_{k}^{2} \sum_{n^{\prime}=-\infty}^{\infty} \frac{1}{n^{\prime}+\frac{1}{2}} \frac{n^{\prime}-n+\frac{1}{2}}{\left(n^{\prime}-n+\frac{1}{2}\right)^{2}+\left(\frac{\beta \epsilon_{k}}{2 \pi}\right)^{2}},
\end{aligned}
$$


everything else simply being a constant prefactor. Splitting this sum into an $n^{\prime}<0$ part (sending $n^{\prime} \rightarrow-n^{\prime}-1$ ) and an $n^{\prime} \geq 0$ part, it is essentially identical to Eq. A.82 with $\Gamma \rightarrow 0$. Consequently, the above terms do not contain a linear term. The linear contribution of the component $C_{55}$ can thus be calculated from the remaining six terms:

$$
\begin{aligned}
C_{55}(\tau) \cong & \frac{\left(\pi v_{F}\right)^{2}}{4 L_{k, k^{\prime}, k^{\prime \prime}, k^{\prime \prime \prime}}^{2}}\left(\epsilon_{k^{\prime \prime \prime}}-\epsilon_{k^{\prime \prime}}\right)\left(\epsilon_{k^{\prime}}-\epsilon_{k}\right)\langle a(\tau) a(0)\rangle_{0} \\
& \times\left(\left\langle\psi_{s f, k}^{\dagger}(\tau) \psi_{s f, k^{\prime \prime \prime}}(0)\right\rangle_{0}\left\langle\psi_{s f, k^{\prime}}(\tau) \psi_{s f, k^{\prime \prime}}^{\dagger}(0)\right\rangle_{0}\langle b(\tau) b(0)\rangle_{0}\right. \\
& -\left\langle\psi_{s f, k}^{\dagger}(\tau) \psi_{s f, k^{\prime \prime}}^{\dagger}(0)\right\rangle_{0}\left\langle\psi_{s f, k^{\prime}}(\tau) \psi_{s f, k^{\prime \prime \prime}}(0)\right\rangle_{0}\langle b(\tau) b(0)\rangle_{0} \\
& +\left\langle\psi_{s f, k}^{\dagger}(\tau) \psi_{s f, k^{\prime \prime \prime}}(0)\right\rangle_{0}\left\langle\psi_{s f, k^{\prime}}(\tau) b(0)\right\rangle_{0}\left\langle\psi_{s f, k^{\prime \prime}}^{\dagger}(0) b(\tau)\right\rangle_{0} \\
& -\left\langle\psi_{s f, k}^{\dagger}(\tau) \psi_{s f, k^{\prime \prime}}^{\dagger}(0)\right\rangle_{0}\left\langle\psi_{s f, k^{\prime}}(\tau) b(0)\right\rangle_{0}\left\langle\psi_{s f, k^{\prime \prime \prime}}(0) b(\tau)\right\rangle_{0} \\
& +\left\langle\psi_{s f, k}^{\dagger}(\tau) b(0)\right\rangle_{0}\left\langle\psi_{s f, k^{\prime}}(\tau) \psi_{s f, k^{\prime \prime}}^{\dagger}(0)\right\rangle_{0}\left\langle\psi_{s f, k^{\prime \prime \prime}}(0) b(\tau)\right\rangle_{0} \\
& \left.-\left\langle\psi_{s f, k}^{\dagger}(\tau) b(0)\right\rangle_{0}\left\langle\psi_{s f, k^{\prime}}(\tau) \psi_{s f, k^{\prime \prime \prime}}(0)\right\rangle_{0}\left\langle\psi_{s f, k^{\prime \prime}}^{\dagger}(0) b(\tau)\right\rangle_{0}\right) .
\end{aligned}
$$

At this point, it is a matter of plugging in the propagators from Sec. 4.2 .3 and simplifying the result, mostly by relabelling indices and using that any term that is odd in any of the momenta vanishes upon integration. A straightforward but very lengthy calculation leads to the following result:

$$
\begin{aligned}
& C_{55}\left(i \Omega_{n}\right) \\
& \cong-\frac{i}{16 \hbar \beta^{3}} \int_{-\Lambda}^{\Lambda} \mathrm{d} \epsilon_{k} \int_{-\Lambda}^{\Lambda} \mathrm{d} \epsilon_{k^{\prime}} \sum_{n^{\prime}, n^{\prime \prime}, n^{\prime \prime \prime}}\left(\epsilon_{k}+\epsilon_{k^{\prime}}\right)^{2} \frac{1}{i \hbar \omega_{n^{\prime \prime \prime}}-\epsilon_{k}} \frac{1}{i \hbar \omega_{n^{\prime \prime}}-\epsilon_{k^{\prime}}} \\
& \times \frac{1}{i \hbar\left(\omega_{n^{\prime}}+\omega_{n^{\prime \prime}}+\omega_{n^{\prime \prime \prime}}-\Omega_{n}\right)} \frac{1}{\hbar \omega_{n^{\prime}}+\operatorname{sgn}\left(\omega_{n^{\prime}}\right) \Gamma} \\
& +\frac{\Gamma}{16 \pi \hbar \beta^{3}} \int_{-\Lambda}^{\Lambda} \mathrm{d} \epsilon_{k} \int_{-\Lambda}^{\Lambda} \mathrm{d} \epsilon_{k^{\prime}} \int_{-\Lambda}^{\Lambda} \mathrm{d} \epsilon_{k^{\prime \prime}} \sum_{n^{\prime}, n^{\prime \prime}, n^{\prime \prime \prime}}\left(\epsilon_{k}+\epsilon_{k^{\prime}}\right)\left(\epsilon_{k}+\epsilon_{k^{\prime \prime}}\right) \frac{1}{i \hbar \omega_{n^{\prime \prime \prime}}-\epsilon_{k}} \\
& \times\left(\frac{1}{i \hbar \omega_{n^{\prime}}-\epsilon_{k^{\prime \prime}}}-\frac{1}{i \hbar \omega_{n^{\prime \prime}}-\epsilon_{k^{\prime \prime}}}\right) \frac{1}{i \hbar \omega_{n^{\prime \prime}}-\epsilon_{k^{\prime}}} \frac{1}{i \hbar\left(\omega_{n^{\prime}}+\omega_{n^{\prime \prime}}+\omega_{n^{\prime \prime \prime}}-\Omega_{n}\right)} \\
& \times \frac{1}{\hbar \omega_{n^{\prime}}+\operatorname{sgn}\left(\omega_{n^{\prime}}\right) \Gamma} \frac{1}{\hbar \omega_{n^{\prime \prime}}+\operatorname{sgn}\left(\omega_{n^{\prime \prime}}\right) \Gamma},
\end{aligned}
$$


where the first two lines can be identified as $C_{44}\left(i \Omega_{n}\right)$, see Eq. (6.45). Splitting the sum from Eq. (6.45) into two parts, we recognize that $C_{44}$ can be evaluated by using Eq. A.82, only with $\Gamma \rightarrow 0$ in the numerators on the left-hand side and $(1-i \overline{\Gamma / 2 \epsilon}) \rightarrow 1$ on the right-hand side. Going through the familiar procedure to evaluate the sum and expanding the result in $n$, we obtain

$$
\begin{aligned}
C_{44}\left(i \Omega_{n}\right)= & \text { const. }+\frac{\beta}{8 \pi^{2} \hbar} \int_{-\Lambda}^{\Lambda} \mathrm{d} \epsilon \frac{\epsilon^{3}}{\tanh (\beta \epsilon)} \frac{\frac{\beta \Gamma}{2 \pi}}{\left(\frac{\beta \Gamma}{2 \pi}\right)^{2}+\left(\frac{\beta \epsilon}{\pi}\right)^{2}} \\
& \times\left[\psi^{(1)}\left(\frac{1}{2}-\frac{i \beta \epsilon}{\pi}\right)+\psi^{(1)}\left(\frac{1}{2}+\frac{i \beta \epsilon}{\pi}\right)\right] n+\mathcal{O}\left(n^{2}\right) .
\end{aligned}
$$

Since we are interested in the NFL fixed point, we furthermore expand to lowest order in $T / T_{K} \sim 1 / \beta \Gamma$, allowing us to evaluate the remaining integral:

$$
C_{44}\left(i \Omega_{n}\right)=\text { const. }+\frac{\pi^{4} \Omega_{n}}{256 \beta^{2}} \frac{1}{\beta \Gamma}+\mathcal{O}\left(\Omega_{n}^{2}, 1 /(\beta \Gamma)^{2}\right) .
$$

Recognizing that $\beta \Gamma \rightarrow \infty$ at the NFL fixed point, we conclude that $C_{44}$ does not have a linear term in $\Omega_{n}$ at this point.

For the remaining terms, we first evaluate the sum over $n^{\prime \prime \prime}$ and the integrals over $\epsilon_{k^{\prime}}$ and $\epsilon_{k^{\prime \prime}}$ while keeping $\Lambda$ finite. Taking into account that $C_{44}$ can be discarded at the NFL fixed point, $C_{55}$ becomes

$$
\begin{aligned}
C_{55}\left(i \Omega_{n}\right) \cong & -\frac{\Gamma}{64 \pi \hbar \beta^{2}} \int_{-\Lambda}^{\Lambda} \mathrm{d} \epsilon_{k} \sum_{n^{\prime}, n^{\prime \prime}} \tanh \left(\frac{\beta \epsilon_{k}}{2}\right) \frac{1}{i \hbar\left(\omega_{n^{\prime}}+\omega_{n^{\prime \prime}}-\Omega_{n}\right)+\epsilon_{k}} \\
& \times \frac{1}{\hbar \omega_{n^{\prime}}+\operatorname{sgn}\left(\omega_{n^{\prime}}\right) \Gamma} \frac{1}{\hbar \omega_{n^{\prime \prime}}+\operatorname{sgn}\left(\omega_{n^{\prime \prime}}\right) \Gamma} \\
& \times\left(\left(\epsilon_{k}+i \hbar \omega_{n^{\prime}}\right)\left(2 \arctan \left(\frac{\hbar \omega_{n^{\prime}}}{\Lambda}\right)-\operatorname{sgn}\left(\omega_{n^{\prime}}\right) \pi\right)\right. \\
& \left.-\left(\epsilon_{k}+i \hbar \omega_{n^{\prime \prime}}\right)\left(2 \arctan \left(\frac{\hbar \omega_{n^{\prime \prime}}}{\Lambda}\right)-\operatorname{sgn}\left(\omega_{n^{\prime \prime}}\right) \pi\right)\right)^{2} .
\end{aligned}
$$

This is as far as we will go before turning to numerical methods.

\section{- $C_{55}$ : numerical evaluation}

In order to calculate Eq. A.93 numerically at the NFL fixed point, we first switch to dimensionless variables. In particular, we define $\epsilon \equiv \epsilon_{k} / \Gamma$, $\omega^{\prime} \equiv \hbar \omega_{n^{\prime}} / \Gamma$ and $\omega^{\prime \prime} \equiv \hbar \omega_{n^{\prime \prime}} / \Gamma$. This choice allows us to take the continuum 
limit of the sums over the Matsubara frequencies: the step sizes are $\Delta \omega^{\prime}=$ $\Delta \omega^{\prime \prime}=2 \pi / \beta \Gamma \rightarrow 0$, such that we can write

$$
\sum_{n^{\prime}=-\infty}^{\infty} \rightarrow \frac{\beta \Gamma}{2 \pi} \int_{-\infty}^{\infty} \mathrm{d} \omega^{\prime},
$$

and similarly for $\omega_{n^{\prime \prime}}$. In terms of these dimensionless variables, we have

$$
\begin{aligned}
C_{55}\left(i \Omega_{n}\right) \cong & -\frac{\Gamma^{3}}{256 \pi^{3} \hbar} \int_{-\Lambda / \Gamma}^{\Lambda / \Gamma} \mathrm{d} \epsilon \int_{-\infty}^{\infty} \mathrm{d} \omega^{\prime} \int_{-\infty}^{\infty} \mathrm{d} \omega^{\prime \prime} \tanh \left(\frac{\beta \Gamma \epsilon}{2}\right) \\
& \times \frac{1}{i\left(\omega^{\prime}+\omega^{\prime \prime}-\frac{\hbar \Omega_{n}}{\Gamma}\right)+\epsilon} \frac{1}{\omega^{\prime}+\operatorname{sgn}\left(\omega^{\prime}\right)} \frac{1}{\omega^{\prime \prime}+\operatorname{sgn}\left(\omega^{\prime \prime}\right)} \\
& \times\left(\left(\epsilon+i \omega^{\prime}\right)\left(2 \arctan \left(\frac{\omega^{\prime}}{\Lambda / \Gamma}\right)-\operatorname{sgn}\left(\omega^{\prime}\right) \pi\right)\right. \\
& \left.-\left(\epsilon+i \omega^{\prime \prime}\right)\left(2 \arctan \left(\frac{\omega^{\prime \prime}}{\Lambda / \Gamma}\right)-\operatorname{sgn}\left(\omega^{\prime \prime}\right) \pi\right)\right)^{2} .
\end{aligned}
$$

We can now treat this as a function of $\hbar \Omega_{n} / \Gamma$ that depends on the two parameters $\Lambda / \Gamma$ and $\beta \Gamma$. The former parameter should be interpreted as large but finite, while the latter is sent to infinity at the NFL fixed point, such that we can write $\tanh (\beta \Gamma \epsilon / 2) \rightarrow \operatorname{sgn}(\epsilon)$. Fixing the parameter $\Lambda / \Gamma$ (i.e. the ratio of the cut-off energy scale to the Kondo energy scale), we have numerically calculated Eq. A.95) for many small values of the dimensionless frequency $\hbar \Omega_{n} / \Gamma$, leading to the plots shown in Fig. 6.1 of the main text. Note that Eq. A.95) with $\tanh (\beta \Gamma \epsilon / 2) \rightarrow \operatorname{sgn}(\epsilon)$ (i.e. the NFL fixed point) is valid for finite temperatures, meaning that this component is independent of the temperature $T$ for any finite temperatures $T \ll T_{K}$. This is different from the qualitative behaviour of the contributing component $C_{11}$ from Eq. 6.37), which is proportional to $T^{2}$ in this regime.

\section{A.10 Self-energy away from the Emery-Kivelson point}

Below we derive the diagrammatic expression from Eq. 8.5 explicitly. To do so, we will use the fact that $\left\langle T_{\tau} b(\tau) a\left(\tau^{\prime}\right)\right\rangle_{0}$ is proportional to the magnetic field $B$ and therefore vanishes if $T^{*}=0$, significantly simplifying the process. From the interaction Hamiltonian from Eq. 8.3) together with Wick's 
theorem, it follows that the partition function is given by

$$
\begin{aligned}
Z^{\text {full }}= & Z\left(1-\frac{i \lambda}{\hbar L} \int_{0}^{\hbar \beta} \mathrm{d} \tau\left\langle b(\tau) a(\tau) \sum_{k, k^{\prime}}: \psi_{s, k}^{\dagger}(\tau) \psi_{s, k^{\prime}}(\tau):\right\rangle_{0}\right. \\
& -\frac{\lambda^{2}}{2 \hbar^{2} L^{2}} \int_{0}^{\hbar \beta} \mathrm{d} \tau \int_{0}^{\hbar \beta} \mathrm{d} \tau^{\prime}\left\langle T_{\tau} b(\tau) a(\tau) \sum_{k, k^{\prime}}: \psi_{s, k}^{\dagger}(\tau) \psi_{s, k^{\prime}}(\tau):\right. \\
& \left.\left.\times b\left(\tau^{\prime}\right) a\left(\tau^{\prime}\right) \sum_{k^{\prime \prime}, k^{\prime \prime \prime}}: \psi_{s, k^{\prime \prime}}^{\dagger}\left(\tau^{\prime}\right) \psi_{s, k^{\prime \prime \prime}}\left(\tau^{\prime}\right):\right\rangle_{0}+\mathcal{O}\left(\lambda^{3}\right)\right) \\
= & Z\left(1+\frac{\lambda^{2}}{2 \hbar^{2} L^{2}} \int_{0}^{\hbar \beta} \mathrm{d} \tau \int_{0}^{\hbar \beta} \mathrm{d} \tau^{\prime} D_{a a}\left(\tau-\tau^{\prime}\right) D_{b b}\left(\tau-\tau^{\prime}\right)\right. \\
& \left.\times \sum_{k, k^{\prime}}\left(G_{s, k}(0) G_{s, k^{\prime}}(0)-G_{s, k}\left(\tau-\tau^{\prime}\right) G_{s, k^{\prime}}\left(\tau^{\prime}-\tau\right)\right)+\mathcal{O}\left(\lambda^{4}\right)\right) \\
\equiv & Z \times Z^{\prime} .
\end{aligned}
$$

We also note that $\sum_{k} G_{s, k}(0)=0$, see Appendix A.8.

With this information, the full propagator of interest (i.e. $\left.D_{b b}^{\text {full }}(\tau)\right)$ can be calculated. Using that $D_{a a / b b}(\tau)=-D_{a a / b b}(-\tau)$ (which follows directly from the fact that $D_{a a / b b}\left(i \omega_{n}\right)$ are both odd in $\omega_{n}$, see Eqs. (6.13) and (6.38) and utilizing the same methods as above, we find

$$
\begin{aligned}
& D_{b b}^{\text {full }}\left(\tau-\tau^{\prime}\right) \\
& =\frac{1}{Z^{\prime}}\left(D_{b b}\left(\tau-\tau^{\prime}\right)+\frac{\lambda^{2}}{2 \hbar^{2} L^{2}} \int_{0}^{\hbar \beta} \mathrm{d} \tau^{\prime \prime} \int_{0}^{\hbar \beta} \mathrm{d} \tau^{\prime \prime \prime}\left\langle T_{\tau} b(\tau) b\left(\tau^{\prime}\right) b\left(\tau^{\prime \prime}\right) a\left(\tau^{\prime \prime}\right) b\left(\tau^{\prime \prime \prime}\right) a\left(\tau^{\prime \prime \prime}\right)\right.\right. \\
& \left.\left.\quad \times \sum_{k, k^{\prime}}: \psi_{s, k}^{\dagger}\left(\tau^{\prime \prime}\right) \psi_{s, k^{\prime}}\left(\tau^{\prime \prime}\right): \sum_{k^{\prime \prime}, k^{\prime \prime \prime}}: \psi_{s, k^{\prime \prime}}^{\dagger}\left(\tau^{\prime \prime \prime}\right) \psi_{s, k^{\prime \prime \prime}}\left(\tau^{\prime \prime \prime}\right):\right\rangle_{0}+\mathcal{O}\left(\lambda^{4}\right)\right) \\
& =\frac{1}{Z^{\prime}}\left(D_{b b}\left(\tau-\tau^{\prime}\right)-\frac{\lambda^{2}}{2 \hbar^{2} L^{2}} \int_{0}^{\hbar \beta} \mathrm{d} \tau^{\prime \prime} \int_{0}^{\hbar \beta} \mathrm{d} \tau^{\prime \prime \prime} D_{a a}\left(\tau^{\prime \prime}-\tau^{\prime \prime \prime}\right)\right. \\
& \quad \times \sum_{k, k^{\prime}} G_{s, k}\left(\tau^{\prime \prime}-\tau^{\prime \prime \prime}\right) G_{s, k^{\prime}}\left(\tau^{\prime \prime \prime}-\tau^{\prime \prime}\right)\left[D_{b b}\left(\tau-\tau^{\prime}\right) D_{b b}\left(\tau^{\prime \prime}-\tau^{\prime \prime \prime}\right)\right. \\
& \left.\left.\quad+D_{b b}\left(\tau-\tau^{\prime \prime}\right) D_{b b}\left(\tau^{\prime \prime \prime}-\tau^{\prime}\right)-D_{b b}\left(\tau-\tau^{\prime \prime \prime}\right) D_{b b}\left(\tau^{\prime \prime}-\tau^{\prime}\right)\right]+\mathcal{O}\left(\lambda^{4}\right)\right)
\end{aligned}
$$




$$
\begin{aligned}
= & D_{b b}\left(\tau-\tau^{\prime}\right)-\frac{\lambda^{2}}{\hbar^{2} L^{2}} \int_{0}^{\hbar \beta} \mathrm{d} \tau^{\prime \prime} \int_{0}^{\hbar \beta} \mathrm{d} \tau^{\prime \prime \prime} D_{a a}\left(\tau^{\prime \prime}-\tau^{\prime \prime \prime}\right) \sum_{k, k^{\prime}} G_{s, k}\left(\tau^{\prime \prime}-\tau^{\prime \prime \prime}\right) \\
& \times G_{s, k^{\prime}}\left(\tau^{\prime \prime \prime}-\tau^{\prime \prime}\right) D_{b b}\left(\tau-\tau^{\prime \prime}\right) D_{b b}\left(\tau^{\prime \prime \prime}-\tau^{\prime}\right)+\mathcal{O}\left(\lambda^{4}\right) .
\end{aligned}
$$

Finally, we rewrite this in terms of fermionic Matsubara frequencies, similar to Eq. 6.5). Using the imaginary time integrals to obtain Kronecker deltas, we indeed find

$$
D_{b b}^{\text {full }}\left(i \omega_{n}\right)=D_{b b}\left(i \omega_{n}\right)+D_{b b}\left(i \omega_{n}\right) \Sigma\left(i \omega_{n}\right) D_{b b}\left(i \omega_{n}\right)+\mathcal{O}\left(\lambda^{4}\right),
$$

where the self-energy is given by Eq. (8.6). 



\section{Bibliography}

[1] C. Castelnovo, R. Moessner, and S. L. Sondhi, "Magnetic monopoles in spin ice," Nature 451, 42 (2008).

[2] V. Mourik, K. Zuo, S. M. Frolov, S. R. Plissard, E. P. A. M. Bakkers, and L. P. Kouwenhoven, "Signatures of Majorana Fermions in Hybrid Superconductor-Semiconductor Nanowire Devices," Science 336, 1003 (2012).

[3] A. C. Hewson, The Kondo Problem to Heavy Fermions (Cambridge University Press, 1993).

[4] A. Altland and B. D. Simons, Condensed Matter Field Theory (Cambridge University Press, 2006).

[5] T. Giamarchi, "Interactions in Quantum Fluids," arXiv preprint arXiv:1007.1030 (2010),

[6] C. Kittel, Introduction to Solid State Physics (Wiley, 2004).

[7] L. D. Landau, "The Theory of a Fermi Liquid," Sov. Phys. JETP 3, 920 (1957).

[8] L. D. Landau, "Oscillations in a Fermi Liquid," Sov. Phys. JETP 5, 101 (1957).

[9] R. Franz and G. Wiedemann, "Ueber die Wärme-Leitungsfähigkeit der Metalle," Ann. Phys. 165, 497 (1853).

[10] L. Lorenz, "Ueber das Leitungsvermögen der Metalle für Wärme und Electricität," Ann. Phys. 249, 422 (1881).

[11] Z. Iftikhar, S. Jezouin, A. Anthore, U. Gennser, F. D. Parmentier, A. Cavanna, and F. Pierre, "Two-channel Kondo effect and renormalization flow with macroscopic quantum charge states," Nature 526, 233 (2015).

[12] A. Schiller and S. Hershfield, "Solution of an ac Kondo Model," Phys. Rev. Lett. 77, 1821 (1996). 
[13] A. Schiller and S. Hershfield, "Toulouse limit for the nonequilibrium Kondo impurity: Currents, noise spectra, and magnetic properties," Phys. Rev. B 58, 14978 (1998).

[14] J. Kondo, "Resistance Minimum in Dilute Magnetic Alloys," Prog. Theor. Phys. 32, 37 (1964).

[15] P. Nozières, "A "fermi-liquid" description of the Kondo problem at low temperatures," J. Low Temp. Phys. 17, 31 (1974).

[16] P. W. Anderson, "Localized Magnetic States in Metals," Phys. Rev. 124, 41 (1961).

[17] J. R. Schrieffer and P. A. Wolff, "Relation between the Anderson and Kondo Hamiltonians," Phys. Rev. 149, 491 (1966).

[18] W. J. de Haas, J. de Boer, and G. J. van den Berg, "The electrical resistance of gold, copper and lead at low temperatures," Physica 1, 1115 (1934),

[19] P. W. Anderson, "A poor man's derivation of scaling laws for the Kondo problem," J. Phys. C 3, 2436 (1970).

[20] K. G. Wilson, "The renormalization group: Critical phenomena and the Kondo problem," Rev. Mod. Phys. 47, 773 (1975).

[21] D. J. Gross and F. Wilczek, "Ultraviolet Behavior of Non-Abelian Gauge Theories," Phys. Rev. Lett. 30, 1343 (1973).

[22] G. 't Hooft, "When was asymptotic freedom discovered? or The rehabilitation of quantum field theory," Nucl. Phys. B (Proc. Suppl.) 74, 413 (1999).

[23] S. M. Cronenwett, T. H. Oosterkamp, and L. P. Kouwenhoven, "A Tunable Kondo Effect in Quantum Dots," Science 281, 540 (1998).

[24] L. P. Kouwenhoven and L. I. Glazman, "Revival of the Kondo effect," Phys. World 14, 33 (2001)

[25] P. B. Wiegmann, "Exact solution of the s-d exchange model (Kondo problem)," J. Phys. C 14, 1463 (1981).

[26] N. Andrei, "Diagonalization of the Kondo Hamiltonian," Phys. Rev. Lett. 45, 379 (1980). 
[27] P. Nozières and A. Blandin, "Kondo effect in real metals," J. Phys. France 41, 193 (1980).

[28] I. Affleck and A. W. W. Ludwig, "Critical theory of overscreened Kondo fixed points," Nucl. Phys. B 360, 641 (1991).

[29] I. Affleck and A. W. W. Ludwig, "Exact conformal-field-theory results on the multichannel Kondo effect: Single-fermion Green's function, selfenergy, and resistivity," Phys. Rev. B 48, 7297 (1993).

[30] T. Giamarchi, Quantum Physics in One Dimension (Clarendon press, 2003).

[31] A. K. Mitchell, K. G. L. Pedersen, P. Hedegård, and J. Paaske, "Kondo blockade due to quantum interference in single-molecule junctions," Nat. Comm. 8, 15210 (2017).

[32] D. Goldhaber-Gordon, H. Shtrikman, D. Mahalu, D. Abusch-Magder, U. Meirav, and M. A. Kastner, "Kondo effect in a single-electron transistor," Nature 391, 156 (1998)

[33] R. M. Potok, I. G. Rau, H. Shtrikman, Y. Oreg, and D. GoldhaberGordon, "Observation of the two-channel Kondo effect," Nature 446, 167 (2007).

[34] A. J. Keller, L. Peeters, C. P. Moca, I. Weymann, D. Mahalu, V. Umansky, G. Zaránd, and D. Goldhaber-Gordon, "Universal Fermi liquid crossover and quantum criticality in a mesoscopic system," Nature 526, 237 (2015),

[35] A. K. Mitchell, L. A. Landau, L. Fritz, and E. Sela, "Universality and Scaling in a Charge Two-Channel Kondo Device," Phys. Rev. Lett. 116, 157202 (2016).

[36] K. A. Matveev, "Coulomb blockade at almost perfect transmission," Phys. Rev. B 51, 1743 (1995).

[37] A. Furusaki and K. A. Matveev, "Theory of strong inelastic cotunneling," Phys. Rev. B 52, 16676 (1995).

[38] N. Andrei and C. Destri, "Solution of the Multichannel Kondo Problem," Phys. Rev. Lett. 52, 364 (1984). 
[39] M. Pustilnik, L. Borda, L. I. Glazman, and J. von Delft, "Quantum phase transition in a two-channel-Kondo quantum dot device," Phys. Rev. B 69, 115316 (2004).

[40] D. L. Cox and A. Zawadowski, "Exotic Kondo effects in metals: Magnetic ions in a crystalline electric field and tunnelling centres," Adv. Phys. 47, 599 (1998).

[41] I. Affleck, A. W. W. Ludwig, H.-B. Pang, and D. L. Cox, "Relevance of anisotropy in the multichannel Kondo effect: Comparison of conformal field theory and numerical renormalization-group results," Phys. Rev. B 45, 7918 (1992).

[42] A. K. Mitchell and E. Sela, "Universal low-temperature crossover in two-channel Kondo models," Phys. Rev. B 85, 235127 (2012)

[43] V. J. Emery and S. Kivelson, "Mapping of the two-channel Kondo problem to a resonant-level model," Phys. Rev. B 46, 10812 (1992),

[44] G. Toulouse, "Infinite- $U$ Anderson Hamiltonian for Dilute Alloys," Phys. Rev. B 2, 270 (1970).

[45] K. Majumdar, A. Schiller, and S. Hershfield, "Nonequilibrium Kondo impurity: Perturbation about an exactly solvable point," Phys. Rev. B 57, 2991 (1998).

[46] Y. Meir and N. S. Wingreen, "Landauer formula for the current through an interacting electron region," Phys. Rev. Lett. 68, 2512 (1992).

[47] A. Kamenev, Field Theory of Non-Equilibrium Systems (Cambridge University Press, 2011).

[48] T. A. Costi and V. Zlatić, "Thermoelectric transport through strongly correlated quantum dots," Phys. Rev. B 81, 235127 (2010).

[49] E. Sela, A. K. Mitchell, and L. Fritz, "Exact Crossover Green Function in the Two-Channel and Two-Impurity Kondo Models," Phys. Rev. Lett. 106, 147202 (2011).

[50] D. Sénéchal, "An Introduction to Bosonization," in Theoretical Methods for Strongly Correlated Electrons (Springer, 2004) pp. 139-186.

[51] A. O. Gogolin, A. A. Nersesyan, and A. M. Tsvelik, Bosonization and Strongly Correlated Systems (Cambridge University Press, 2004). 
[52] A.-P. Jauho, N. S. Wingreen, and Y. Meir, "Time-dependent transport in interacting and noninteracting resonant-tunneling systems," Phys. Rev. B 50, 5528 (1994).

[53] C. P. Moca, A. Roman, and D. C. Marinescu, "Thermal conductance of a two-channel Kondo model," Phys. Rev. B 83, 245308 (2011).

[54] M. S. Green, "Markoff Random Processes and the Statistical Mechanics of Time-Dependent Phenomena," J. Chem. Phys. 20, 1281 (1952).

[55] R. Kubo, "Statistical-Mechanical Theory of Irreversible Processes. I. General Theory and Simple Applications to Magnetic and Conduction Problems," J. Phys. Soc. Jpn. 12, 570 (1957).

[56] J. M. Luttinger, "Theory of Thermal Transport Coefficients," Phys. Rev. 135, A1505 (1964)

[57] E. Sela and I. Affleck, "Nonequilibrium Transport through Double Quantum Dots: Exact Results near a Quantum Critical Point," Phys. Rev. Lett. 102, 047201 (2009).

[58] E. Sela and I. Affleck, "Nonequilibrium critical behavior for electron tunneling through quantum dots in an Aharonov-Bohm circuit," Phys. Rev. B 79, 125110 (2009).

[59] R. Scheibner, H. Buhmann, D. Reuter, M. N. Kiselev, and L. W. Molenkamp, "Thermopower of a Kondo Spin-Correlated Quantum Dot," Phys. Rev. Lett. 95, 176602 (2005).

[60] A. Garg, D. Rasch, E. Shimshoni, and A. Rosch, "Large Violation of the Wiedemann-Franz Law in Luttinger Liquids," Phys. Rev. Lett. 103, $096402(2009)$.

[61] R. Mahajan, M. Barkeshli, and S. A. Hartnoll, "Non-Fermi liquids and the Wiedemann-Franz law," Phys. Rev. B 88, 125107 (2013).

[62] R. Žitko, J. Mravlje, A. Ramšak, and T. Rejec, "Spin thermopower in the overscreened Kondo model," New J. Phys. 15, 105023 (2013).

[63] J. Zaanen, Y. Liu, Y.-W. Sun, and K. Schalm, Holographic Duality in Condensed Matter Physics (Cambridge University Press, 2015).

[64] T. K. T. Nguyen and M. N. Kiselev, "Seebeck effect on a weak link between Fermi and non-Fermi liquids," Phys. Rev. B 97, 085403 (2018). 
[65] A. Lavasani, D. Bulmash, and S. Das Sarma, "Wiedemann-Franz law and Fermi liquids," Phys. Rev. B 99, 085104 (2019).

[66] B. Kubala, J. König, and J. Pekola, "Violation of the WiedemannFranz law in a Single-Electron Transistor," Phys. Rev. Lett. 100, 066801 (2008).

[67] B. Dutta, J. T. Peltonen, D. S. Antonenko, M. Meschke, M. A. Skvortsov, B. Kubala, J. König, C. B. Winkelmann, H. Courtois, and J. P. Pekola, "Thermal Conductance of a Single-Electron Transistor," Phys. Rev. Lett. 119, 077701 (2017).

[68] J. Crossno, J. K. Shi, K. Wang, X. Liu, A. Harzheim, A. Lucas, S. Sachdev, P. Kim, T. Taniguchi, K. Watanabe, T. A. Ohki, and K. C. Fong, "Observation of the Dirac fluid and the breakdown of the Wiedemann-Franz law in graphene," Science 351, 1058 (2016).

[69] J. P. Bergfield and C. A. Stafford, "Thermoelectric Signatures of Coherent Transport in Single-Molecule Heterojunctions," Nano Lett. 9, 3072 (2009).

[70] D. Bernard and B. Doyon, "Energy flow in non-equilibrium conformal field theory," J. Phys. A 45, 362001 (2012).

[71] Z. Iftikhar, A. Anthore, A. K. Mitchell, F. D. Parmentier, U. Gennser, A. Ouerghi, A. Cavanna, C. Mora, P. Simon, and F. Pierre, "Tunable quantum criticality and super-ballistic transport in a "charge" Kondo circuit," Science 360, 1315 (2018).

[72] H. Zheng, S. Florens, and H. U. Baranger, "Transport signatures of Majorana quantum criticality realized by dissipative resonant tunneling," Phys. Rev. B 89, 235135 (2014).

[73] M. Pustilnik, B. van Heck, R. M. Lutchyn, and L. I. Glazman, "Quantum Criticality in Resonant Andreev Conduction," Phys. Rev. Lett. 119, $116802(2017)$.

[74] L. A. Landau, E. Cornfeld, and E. Sela, "Charge Fractionalization in the Two-Channel Kondo Effect," Phys. Rev. Lett. 120, 186801 (2018).

[75] O. Zachar, S. A. Kivelson, and V. J. Emery, "Exact Results for a 1D Kondo Lattice from Bosonization," Phys. Rev. Lett. 77, 1342 (1996). 
[76] P. Coleman, A. Georges, and A. M. Tsvelik, "Reflections on the onedimensional realization of odd-frequency pairing," J. Phys. Condens. Matter 9, 345 (1997).

[77] M. Fabrizio and A. O. Gogolin, "Toulouse limit for the overscreened four-channel Kondo problem," Phys. Rev. B 50, 17732 (1994).

[78] J. von Delft and H. Schoeller, "Bosonization for beginners - refermionization for experts," Ann. Phys. 7, 225 (1998).

[79] W. Izumida, O. Sakai, and Y. Shimizu, "Many Body Effects on Electron Tunneling through Quantum Dots in an Aharonov-Bohm Circuit," J. Phys. Soc. Jpn. 66, 717 (1997). 



\section{Nederlandse samenvatting}

Het vakgebied waar dit proefschrift aan bijdraagt staat bekend als de fysica van de gecondenseerde materie. Deze richting houdt zich bezig met de eigenschappen van materie, zoals de vraag waarom sommige materialen wel elektriciteit geleiden en andere materialen niet. Het goed begrijpen van materiaaleigenschappen kan leiden tot zeer nuttige toepassingen. Denk hierbij bijvoorbeeld aan halfgeleiders en de uitvinding van de transistor, die aan de basis staat van vrijwel alle moderne technologie. $\mathrm{Nu}$ is de term "materie" vrij breed; wij beperken ons dan ook tot kristallijne vaste stoffen. Met een kristallijne vaste stof bedoelen we een vaste stof die op het allerkleinste niveau (oftewel de atomen waar de stof uit bestaat) een kristalstructuur hebben, zoals de meeste metalen, diamant en keukenzout. In dit soort materialen kunnen de elektronen rondspringen tussen verschillende atomen, en de manier waarop dit gebeurt is bepalend voor de elektrische eigenschappen van het materiaal. Vanwege de zeer kleine schaal van de elektronen worden deze eigenschappen gedicteerd door de kwantummechanica. De kwantummechanica vertelt ons dat elektronen niet zomaar hun gang kunnen gaan, maar zich slechts in een discreet aantal toestanden kunnen bevinden. Welke toestanden dit precies zijn, hangt af van het soort atomen en de kristalstructuur van het materiaal. Wanneer er veel goed bereikbare toestanden voor de elektronen zijn, hebben de elektronen (en dus ook hun elektrische lading) de vrijheid om door het materiaal heen te stromen en is het dus een geleider; wanneer dit niet het geval is, spreken we van een isolator.

\section{Collectieve excitaties}

Het bovenstaande is echter niet het volledige verhaal. Om een indruk te krijgen van wat er allemaal nog meer mogelijk is in deze vaste stoffen, moeten we ons realiseren dat we fundamenteel gezien te maken hebben met zeer grote hoeveelheden deeltjes en dus ook hun gezamenlijke eigenschappen. Los van elkaar (dat wil zeggen de individuele atoomkernen en de elektronen) zal hun gedrag relatief eenvoudig zijn, zoals beschreven wordt door het Standaardmodel van de deeltjesfysica. Grote hoeveelheden deeltjes kunnen echter ook gezamenlijk gedrag vertonen dat niet bestaat op het niveau van individuele 
deeltjes. Als voorbeeld kun je denken aan een grote hoeveelheid balletjes op een tafel die samen een rooster vormen, waarbij elk balletje met zijn naaste buren is verbonden met een veer. Wanneer je één van de balletjes plotseling een zet geeft, zal er dankzij alle verbindingen een trilling door het rooster heen gaan. In zekere zin gedragen zulke trillingen zich (net als de balletjes zelf) als wisselwerkende deeltjes: de trillingen hebben een vrij goed gedefinieerde positie en snelheid, en wanneer twee verschillende trillingen tegen elkaar op botsen, zullen ze elkaar sterk beïnvloeden. Cruciaal bij deze voortbewegende golven is dat ze niet bestaan zouden hebben als de balletjes niet met elkaar verbonden waren geweest. Het ontstaan van dergelijke collectieve excitaties in systemen met grote hoeveelheden wisselwerkende deeltjes wordt ook wel emergentie genoemd en speelt een cruciale rol in de fysica van de gecondenseerde materie. De kwantummechanica vertelt ons verder dat de typisch golfachtige collectieve excitaties inderdaad ook geïnterpreteerd kunnen worden als deeltjes. Deze emergente effectieve deeltjes zijn echter geen fundamentele deeltjes, waardoor ze bijzondere eigenschappen kunnen hebben die niet voorkomen in het Standaardmodel. Een voorbeeld hiervan zijn elektron-achtige deeltjes die elkaar compleet vernietigen wanneer ze met soortgenoten in contact komen; deze zogeheten "Majorana fermionen" spelen een belangrijke rol in dit proefschrift. Dankzij het bestaan van dit soort collectieve excitaties is de gecondenseerde materie een rijk en veelbelovend vakgebied voor het bestuderen van exotische deeltjes.

In dit proefschrift onderzoeken we in het bijzonder sterk wisselwerkende elektronen in een kristalrooster van atomen, welke mogelijk ongebruikelijke collectieve excitaties kunnen ondersteunen. Dit doen we strikt bij lage temperaturen. Bij dergelijke lage temperaturen zijn er maar weinig fundamentele excitaties, oftewel elektronen die uit hun evenwichtstoestand gedreven zijn. Deze fundamentele excitaties liggen gemiddeld gezien dus ver van elkaar af, waardoor de wisselwerking tussen verschillende excitaties een verwaarloosbaar klein effect oplevert. Elke excitatie zal echter nog wel sterk wisselwerken met de deeltjes in zijn directe omgeving. Het blijkt dat een excitatie en zijn directe omgeving vaak samen beschreven kunnen worden als één collectieve excitatie, genaamd een quasideeltje, en dat de verschillende quasideeltjes slechts zwak wisselwerken met elkaar. Hierdoor gedraagt het sterk wisselwerkende materiaal zich alsnog als een zwak wisselwerkend materiaal, met alle gevolgen van dien. De reden dat deze quasideeltjes goed gedefinieerd zijn, is terug te leiden naar het feit dat elektronen zogeheten fermionen zijn. Dit betekent dat twee elektronen zich niet in exact dezelfde toestand kunnen bevinden. Materialen waarvoor een beschrijving in termen van quasideeltjes volstaat, worden ook wel Fermi vloeistoffen genoemd. 
Deze benaming slaat op het feit dat de "soep" van elektronen die door de vaste stof heen bewegen veel weg heeft van een vloeistof. Fermi vloeistoffen zijn te herkennen aan diverse eigenschappen, zoals de kwalitatieve temperatuurafhankelijkheid van de warmtecapaciteit en de compressibiliteit. In dit proefschrift is echter vooral de Wiedemann-Franz wet belangrijk, die stelt dat de verhouding van de warmtegeleidingscoëfficiënt en de temperatuur maal de elektrische conductiviteit van een Fermi vloeistof een universele constante waarde aanneemt. Deze relatie komt voort uit het feit dat warmte en elektrische lading door dezelfde quasideeltjes door het materiaal heen vervoerd worden, en zowel de warmtestroom als de elektrische stroom dus direct evenredig zijn met het aantal vervoerde quasideeltjes.

In tegenstelling tot Fermi vloeistoffen bestaan er ook sterk wisselwerkende materialen waarvoor een beschrijving in termen van zwak wisselwerkende quasideeltjes niet volstaat. Dit soort materialen worden non-Fermi vloeistoffen genoemd, en in veel gevallen worden hun eigenschappen nog niet goed begrepen. Vandaag de dag worden non-Fermi vloeistoffen vooral geïdentificeerd aan de hand van afwijkingen van de Wiedemann-Franz wet. Om de Wiedemann-Franz wet te kunnen testen, is het nodig om te meten wat voor warmtestromen en elektrische stromen er ontstaan in bijzijn van kleine temperatuurgradiënten en spanningsverschillen.

\section{Geleidingseigenschappen van een non-Fermi vloeistof}

Het hoofddoel van dit proefschrift is om een bijdrage te leveren aan het algemene begrip van sterk wisselwerkende elektronen in systemen die zich niet gedragen als een Fermi vloeistof. Dit doen we aan de hand van zogeheten Kondo modellen, gekenmerkt door lokale magnetische onzuiverheden die sterk wisselwerken met de elektronen van het onderliggende materiaal. Hierbij kun je bijvoorbeeld denken aan onzuiverheden in een verder perfect stuk goud, waarbij elke onzuiverheid effectief een heel klein magneetje is. De sterke wisselwerking tussen de onzuiverheden en de nabijgelegen elektronen zorgt ervoor dat deze elektronen de onzuiverheden bij lage temperaturen willen screenen: de elektronen werken samen om een tegengesteld magnetisch veld op te wekken, waarmee ze het magnetische veld van de onzuiverheden op proberen te heffen. Wanneer dit lukt, zullen de onzuiverheden nagenoeg onzichtbaar zijn voor de overige elektronen en is het geheel dus een Fermi vloeistof. We besteden echter vooral veel aandacht aan de experimentele opstelling van Ref. [11], waarin dit screenen minder eenvoudig is. De opstelling bestaat uit enkele los van elkaar staande elektroden die verbonden 
zijn met een metaalachtig "eilandje", welke zich gedraagt als een magnetische onzuiverheid. Deze opstelling heeft bijzondere eigenschappen: er zijn twee elektroden die het eilandje proberen te screenen, maar door de symmetrie van de opstelling is er een conflict tussen de elektroden. De elektroden kunnen namelijk niet beslissen welke van de twee het eilandje moet gaan screenen. Door deze zogeheten frustratie blijven de sterke wisselwerkingen zelfs bij lage temperaturen een hoofdrol spelen en hebben we dus te maken met een non-Fermi vloeistof. Uit eerder onderzoek is gebleken dat dit soort Kondo modellen inderdaad diverse eigenschappen vertonen die bevestigen dat de opstelling een non-Fermi vloeistof is.

Het grootste deel van dit proefschrift is besteed aan theoretisch onderzoek naar de geleidingseigenschappen van de bovengenoemde non-Fermi vloeistof, met name de warmtestroom en de elektrische stroom tussen de twee elektroden ten gevolge van kleine temperatuurgradiënten en voltages. De motivatie om deze specifieke opstelling te bestuderen is het feit dat men buitengewoon veel controle over deze non-Fermi vloeistof heeft, waardoor onze theoretische voorspellingen binnen bereik zijn van bestaande experimenten. Ons onderzoek heeft nieuwe resultaten opgeleverd met betrekking tot de elektrische stroom ten gevolge van tijdsafhankelijke voltages (denk bijvoorbeeld aan wisselstroom) en als belangrijkste de warmtegeleidingscoëfficiënt, die aangeeft hoe goed de opstelling warmte geleidt. Deze resultaten brengen twee belangrijke gevolgen met zich mee voor het algemene begrip van non-Fermi vloeistoffen en Kondo opstellingen. Hieronder bespreken we kort beide van deze consequenties.

Ten eerste hebben we ontdekt dat de Wiedemann-Franz wet behouden is voor de onderzochte Kondo opstelling. Met andere woorden: transport van warmte en elektrische lading zijn direct aan elkaar gerelateerd, wat impliceert dat zowel warmte als elektrische lading door exact dezelfde (quasi)deeltjes gedragen wordt. Dit spreekt zeker niet voor zich vanwege de complexiteit van de opstelling en is ook wat we juist niet zouden verwachten van een non-Fermi vloeistof. Sterker nog: men neemt tegenwoordig vaak aan dat er een één-opéén relatie bestaat tussen afwijkingen van de Wiedemann-Franz wet en nonFermi vloeistoffen. We hebben hier dus een expliciet tegenvoorbeeld voor gevonden, waardoor bewezen is dat deze gebruikelijke aanname niet altijd geldt en het dus nodig is om voorzichtig te zijn met het toepassen ervan.

Ten tweede hebben we een direct verband gevonden tussen de warmtegeleidingscoëfficiënt en de aard van de excitaties in de onderzochte opstelling. Uit eerder theoretisch onderzoek was gebleken dat deze excitaties Majorana fermionen zijn, oftewel (in deze context) effectief "halve" elektronen. Het is echter over het algemeen zeer moeilijk om experimenteel aan te tonen of 
excitaties Majorana fermionen zijn. Wat onze resultaten laten zien, is dat metingen van warmtetransport tussen de twee elektroden kenmerken bloot zullen leggen waarmee de Majorana aard van de excitaties experimenteel aangetoond kan worden. Dankzij de bijzondere eigenschappen van Majorana fermionen zou een dergelijke experimentele bevestiging mogelijk de weg vrij kunnen maken voor nuttige toepassingen in toekomstige technologie.

\section{Tot slot}

Kort samengevat hebben we de geleidingseigenschappen bestudeerd van een specifieke non-Fermi vloeistof, met als doel om een beter begrip te krijgen van het gedrag van sterk wisselwerkende elektronen en hun collectieve excitaties in een kristallijne vaste stof. Als belangrijkste resultaat hebben we gevonden dat één van de meest gangbare kenmerken van een non-Fermi vloeistof, namelijk afwijkingen in de verhouding van de warmtegeleidingscoëfficiënt en de elektrische conductiviteit, niet onfeilbaar is voor het identificeren van nonFermi vloeistoffen. Verder zijn onze theoretische resultaten binnen bereik van bestaande experimenten. Het experimenteel bevestigen van de theoretische resultaten zou een belangrijke stap zijn in het begrijpen van de mogelijke eigenschappen van sterk wisselwerkende materialen. 



\section{Acknowledgements}

Following the tradition, I will now take the opportunity to thank the people who have made it possible for me to write this thesis, or have otherwise been important to me in the context of the academic journey that I have been on for the past four years.

First and foremost, I would like to thank my supervisor Lars. It goes without saying that your guidance, encouragement and optimism have been invaluable. I have learnt a lot from you, and not only regarding physics. Your pragmatic ways have definitely helped me deal with setbacks. Thank you for everything! These past five years working with you have been a pleasure. I would also like to thank my colleagues for making my time at the institute, schools and conferences enjoyable. Honourable mentions go to my more direct colleagues Sonja, Micke and Tycho; I have always enjoyed conversing with you. Even though our paths are diverging, I sincerely hope we meet again someday! And of course, I wish all of you the best of luck with whatever the future holds.

Next, I would like to thank Andrew for our collaboration and for hosting me at University College Dublin for two months, and Emma and Jonas for their support during this time in particular. Emma, I really enjoyed working with you when you were still in Utrecht, and I am grateful for the lasting friendship that this collaboration has led to. Jonas, it was fantastic talking to you about hiking, skiing and (sometimes rather unconventional) philosophical questions.

Finally, I am grateful to my parents, brothers and friends for their interest and support, despite not being involved in the research itself. In this category, special thanks go to my paranymph Jaap, without whom my life would have been strangely devoid of burgers.

What a ride it has been. 



\section{About the author}

Gerwin Antony Ronald van Dalum was born on the 28th of May, 1991 in Nieuwegein, the Netherlands. He attended College de Heemlanden in Houten, specializing in the two profiles "Natuur en Techniek" and "Natuur en Gezondheid" and graduating in 2009. Afterwards, he studied Physics and Astronomy at Utrecht University. He obtained his bachelor's degree cum laude in 2013, with a thesis on standing sound waves in a Bose-Einstein condensate. He continued along this path by enrolling in the master's programme in Theoretical Physics at the same university. This ultimately resulted in a thesis on two separate topics in the field of condensed matter theory, namely phase transitions of the Swedenborgite antiferromagnet and quasiparticle interference in a topological insulator. He graduated cum laude in 2016. In the same year, he started as a $\mathrm{PhD}$ candidate at the Institute for Theoretical Physics in Utrecht under the supervision of dr. Lars Fritz. This dissertation is an account of the knowledge he acquired during the following four years of research. 